\title{
Unraveling the High Activity of Ylide-functionalized Phosphines in Palladium Catalyzed Amination Reactions: A Comparative Study with CyJohnPhos and $\mathrm{PtBu}_{3}$
}

Lennart T. Scharf, Ilja Rodstein, Michelle Schmidt, Thorsten Scherpf and Viktoria H.

Gessner*

Ruhr University Bochum, Faculty of Chemistry and Biochemistry, Chair of Inorganic Chemistry II, Universitätsstrasse 150, 44780 Bochum, Germany.

Email: viktoria.gessner@rub.de

\section{$\underline{\text { Index }}$}

1. Experimental Details 2

1.1 General methods 2

1.2 Preparation of the compounds 2

1.2.1 Preparation of $\mathrm{L} 1_{2} \cdot \mathrm{Pd} \quad 2$

1.2.2 Preparation of $22^{\mathrm{Tol}} \quad 2$

1.2.3 Preparation of $3^{\text {Tol }} 3$

2. NMR spectra 3

3. Catalytic and Kinetic studies $\quad 7$

3.1 Procedure for the BHA with L1, L2 and L3 (Table 1) 7

3.2 Procedure and data of the kinetic studies with keYPhos, L1 7

3.3 Variable time normalization analysis 9

4. Crystal Structure Determination 10

4.1 General information 10

$\begin{array}{lll}4.2 & \text { Crystal structures } & 11\end{array}$

4.2.1 Crystal Structure Determination of $\mathrm{L} 1_{2} \cdot \mathrm{Pd} \quad 11$

4.2.2 Crystal Structure Determination of $22 \quad 15$

4.2.3 Crystal Structure Determination of 3Et 18

5. Computational Details 21

5.1 General 21

5.2 NBO Studies $\quad 21$

5.3 Energies and Coordinates 22

5.3.1 Energies and Coordinates Related to Scheme 1222

5.3.2 Energies and Coordinates Related to Scheme $2 \quad 61$

5.3.3 Energies and Coordinates Related to Scheme 3 (RE) 75

5.3.4 Energies and Coordinates Related to Figure $6 \quad 83$

5.3.5 Energies and Coordinates Related to Scheme 4 108

5.3.6 Energies and Coordinates for Other Aryl Chlorides and Amines 125

5.3.7 Catalytic cycle for Amine $=$ Methylphenylamine and Arylchloride $=p$-F-phenylchloride224

5.3.8 Energies and coordinates related to Table $4 \quad 225$

6. References 240 


\section{Experimental Details}

\subsection{General methods}

All experiments were carried out under a dry, oxygen-free argon atmosphere using standard Schlenk techniques. Involved solvents were dried using an MBraun SPS-800 (THF and pentane) or dried in accordance with standard procedures. ${ }^{1} \mathrm{H},{ }^{13} \mathrm{C}\left\{{ }^{1} \mathrm{H}\right\},{ }^{31} \mathrm{P}\left\{{ }^{1} \mathrm{H}\right\}$ NMR spectra were recorded on Avance-400 spectrometers at $25^{\circ} \mathrm{C}$ if not stated otherwise. All values of the chemical shift are in ppm regarding the $\delta$-scale. All spin-spin coupling constants $(J)$ are printed in Hertz $(\mathrm{Hz})$. To display multiplicities and signal forms correctly the following abbreviations were used: $s=$ singlet, $d=$ doublet, $m=$ multiplet, $d d=$ doublet of doublet, $b r=$ broad signal. Signal assignment was supported by APT, HSQC and HMBC. Elemental analyses were performed on an Elementar vario MICRO-cube elemental analyzer. GC-FID analyses were done with an Agilent 8890 GC System. (tmeda)PdMe ${ }_{2}^{1}$ was prepared according to published procedures. All other reagents were purchased from Umicore, Sigma-Aldrich, ABCR, Rockwood Lithium or Acros Organics and used without further purification. keYPhos (L1) was prepared according to Angew. Chem. Int. Ed. 2019, 58, 3203.

\subsection{Preparation of the compounds}

\subsubsection{Preparation of $\mathrm{L}_{2} \cdot \mathrm{Pd}$}

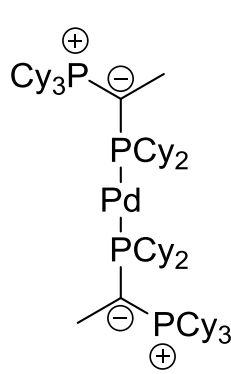

$400 \mathrm{mg}$ ( $0.79 \mathrm{mmol}, 2$ eq.) of ligand $\mathbf{L} 1$ was dissolved with $100 \mathrm{mg}(0.39 \mathrm{mmol}, 1$ eq. $)$ of (tmeda)PdMe 2 in $15 \mathrm{ml}$ of dry THF and stirred overnight. The precipitated solid was filtered off and washed two times with $10 \mathrm{ml}$ of dry THF and $10 \mathrm{ml}$ of dry pentane. The product was dried in vacuo to obtain a light grey solid (200 mg, $0.18 \mathrm{mmol}, 45 \%$ ).

${ }^{1} \mathrm{H}$ NMR (400 MHz, THF-d8) $1.11-2.15(\mathrm{~m}, 110 \mathrm{H}), 3.05\left(\mathrm{~m}, 6 \mathrm{H}, \mathrm{CH}, \mathrm{PC} y_{3}, \mathrm{H} 1\right) \mathrm{ppm} .{ }^{31} \mathrm{P}$ $\left\{{ }^{1} \mathrm{H}\right\}$ NMR $\left(162 \mathrm{MHz}, \mathrm{THF}-\mathrm{d}_{8}\right) \delta=18.9$ (higher order, $\left.P C \mathrm{y}_{2}\right), 30.3$ (higher order, $\left.P C \mathrm{y}_{3}\right)$ ppm. CHNS: Calculated: C: 67.81, H: 10.31. Measured: C: 68.88, H: 10.48 (presumably small amounts of palladium in the isolated product)

\subsubsection{Preparation of $\mathbf{2}_{2}{ }^{\mathrm{Tol}}$}<smiles>Cc1ccc([Pb](Cl)(Cl)P(Cl)(Cl)(Cl)c2ccc(C)cc2)cc1</smiles>

$500 \mathrm{mg}(0.99 \mathrm{mmol}, 1$ eq.) of ligand L1 wwere stirred with $742 \mathrm{mg}$ (1.09 mmol, 1.1 eq.) of $\mathrm{Pd}_{2}(\mathrm{dba})_{3} \times \mathrm{dba}$ in $10 \mathrm{ml}$ of THF for 30 minutes. After filtration of the solution, $1 \mathrm{ml}$ of chlorotoluene was added and the suspension was stirred for 48 hours. The darkyellow precipitate was filtered off with a Schlenk frit and the solid was washed three times with $10 \mathrm{ml}$ of THF. The product was obtained as a dark-yellow solid ( $515 \mathrm{mg}, 0.35 \mathrm{mmol}, 70 \%$ ).

${ }^{1} \mathrm{H}$ NMR $\left(400 \mathrm{MHz}, \mathrm{CD}_{2} \mathrm{Cl}_{2}\right) \delta=1.55\left(\mathrm{dd},{ }^{3} \mathrm{JHP}_{\mathrm{HP}}=12.8 \mathrm{~Hz},{ }^{3} \mathrm{JHP}=9.4 \mathrm{~Hz}, 6 \mathrm{H}, \mathrm{CH}_{3}\right), 0.95-2.08$ (m, 104H, CH + $\left.\mathrm{CH}_{2}, \mathrm{PCy}_{2}+\mathrm{PCy}_{3}\right), 2.14\left(\mathrm{~s}, 6 \mathrm{H}, \mathrm{CH}_{3}, \mathrm{Tolyl}\right), 2.57$ (br, 6H, CH, $\left.\mathrm{PCy}_{3}, \mathrm{H} 1\right), 6.68-6.83$ (m, 4, CH, Tolyl), $7.08-7.23\left(\mathrm{~m}, 4 \mathrm{H}, \mathrm{CH}\right.$, Tolyl) ppm. ${ }^{31} \mathrm{P}\left\{{ }^{1} \mathrm{H}\right\}$ NMR $\left(162 \mathrm{MHz}, \mathrm{CD}_{2} \mathrm{Cl}_{2}\right) \delta=32.5$ (d, $\left.{ }^{2} \mathrm{JPP}_{\mathrm{PP}}=49.6 \mathrm{~Hz}, P C \mathrm{y}_{3}\right), 35.1$ (d, $\left.{ }^{2} \mathrm{JPP}=49.6 \mathrm{~Hz}, P C \mathrm{y}_{2}\right)$ ppm. CHNS: Calculated: $\mathrm{C}: 63.49$, $\mathrm{H}:$ 8.88. Measured: C: $63.18, \mathrm{H}: 8.83$.<smiles>CC1CCCCC1</smiles>

${ }^{13} \mathrm{C}\left\{{ }^{1} \mathrm{H}\right\}$ NMR was not possible due to decomposition of product in dichloromethane and insolubility in all other common organic solvents 


\subsubsection{Preparation of $3^{\text {Tol }}$}<smiles></smiles>

In a J. Young NMR tube, $20 \mathrm{mg}(0.03 \mathrm{mmol})$ of compound $\mathbf{2} 2$ were suspended in $0.6 \mathrm{ml}$ of deuterated THF. $0.1 \mathrm{ml}$ of diethylamine were added and the tube was shaken for $22 \mathrm{~h}$, after which NMR spectroscopic data were recorded. Isolation of the complex failed, because of the back reaction to $\mathbf{2}_{2}{ }^{\text {Tol }}$ during the removal of the solvent (and the amine) in vacuo. Crystals of $3^{\text {Tol }}$ were obtained by over-layering a saturated solution of $\mathbf{2}_{\mathbf{2}}{ }^{\mathrm{Tol}}$ in tetrahydrofurane and diethylamine with pentane.

${ }^{1} \mathrm{H}$ NMR $\left(400 \mathrm{MHz}, \mathrm{THF}-\mathrm{d}_{8}\right) \delta=0.45-2.76\left(\mathrm{~m}, 61 \mathrm{H}, \mathrm{CH}\right.$, PCy2 + PCy $3+\mathrm{CH}_{2, \mathrm{PCy} 2}+\mathrm{PCy} 3+\mathrm{CH}_{3}$, Tolyl, Cy3PEt $)$, $2.96\left(\mathrm{~d},{ }^{3} \mathrm{JHP}_{\mathrm{HP}}=15.0 \mathrm{~Hz}, 4 \mathrm{H}, \mathrm{CH}_{2, \mathrm{HNEt}}\right), 6.62-6.84(\mathrm{~m}, 2 \mathrm{H}, \mathrm{CH}$, Tolyl), $7.28-7.40(\mathrm{~m}, 2 \mathrm{H}, \mathrm{CH}$, Tolyl) ppm. ${ }^{31} \mathrm{P}\left\{{ }^{1} \mathrm{H}\right\}$ NMR $\left(162 \mathrm{MHz}, \mathrm{THF}-d_{8}\right) \delta=15.8-21.9\left(\mathrm{br}, P C \mathrm{y}_{2}\right), 31.0\left(\mathrm{~d},{ }^{2} \mathrm{JPP}_{\mathrm{PP}}=40.9 \mathrm{~Hz}, P C \mathrm{y}_{3}\right) \mathrm{ppm}$.

\section{NMR spectra}

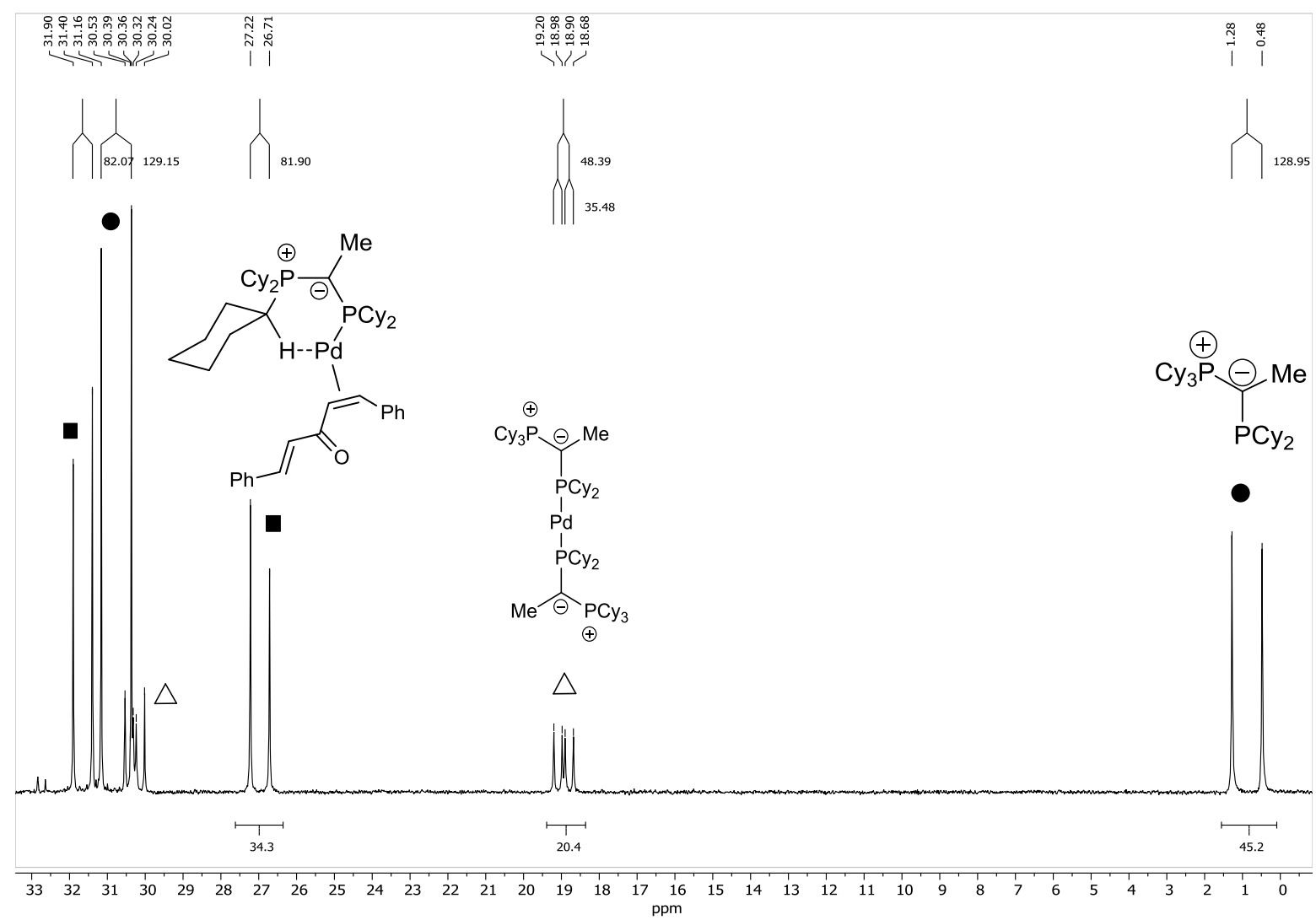

Figure S1. ${ }^{31} \mathrm{P}\left\{{ }^{1} \mathrm{H}\right\}$ NMR spectrum of the reaction mixture of 2 eq. of $L 1$ with 1 eq. of $P d_{2} d b a_{3} x d b a$. 


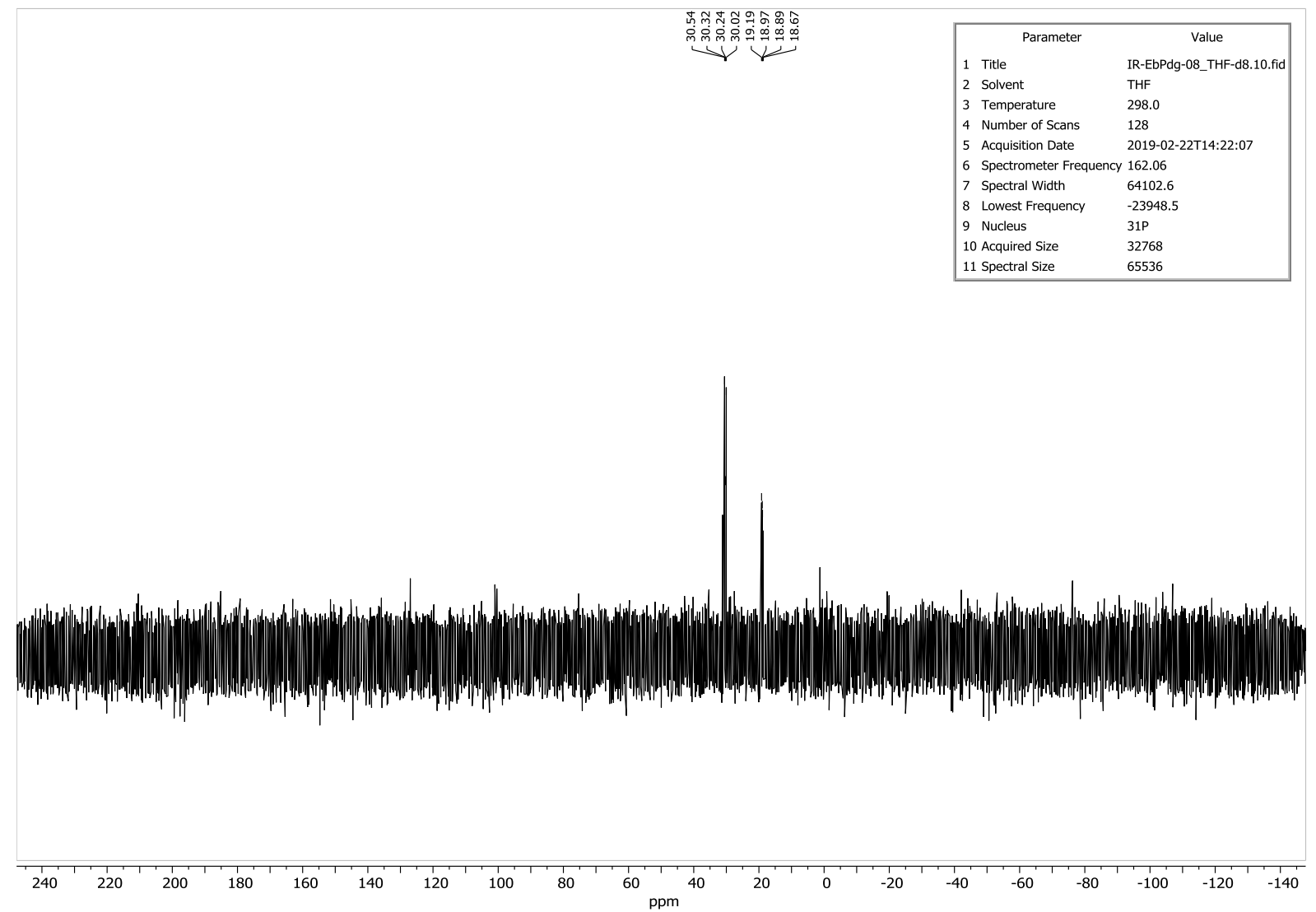

Figure S2a. ${ }^{31} \mathrm{P}\left\{{ }^{1} \mathrm{H}\right\}$ NMR spectrum of L12.Pd.

菺

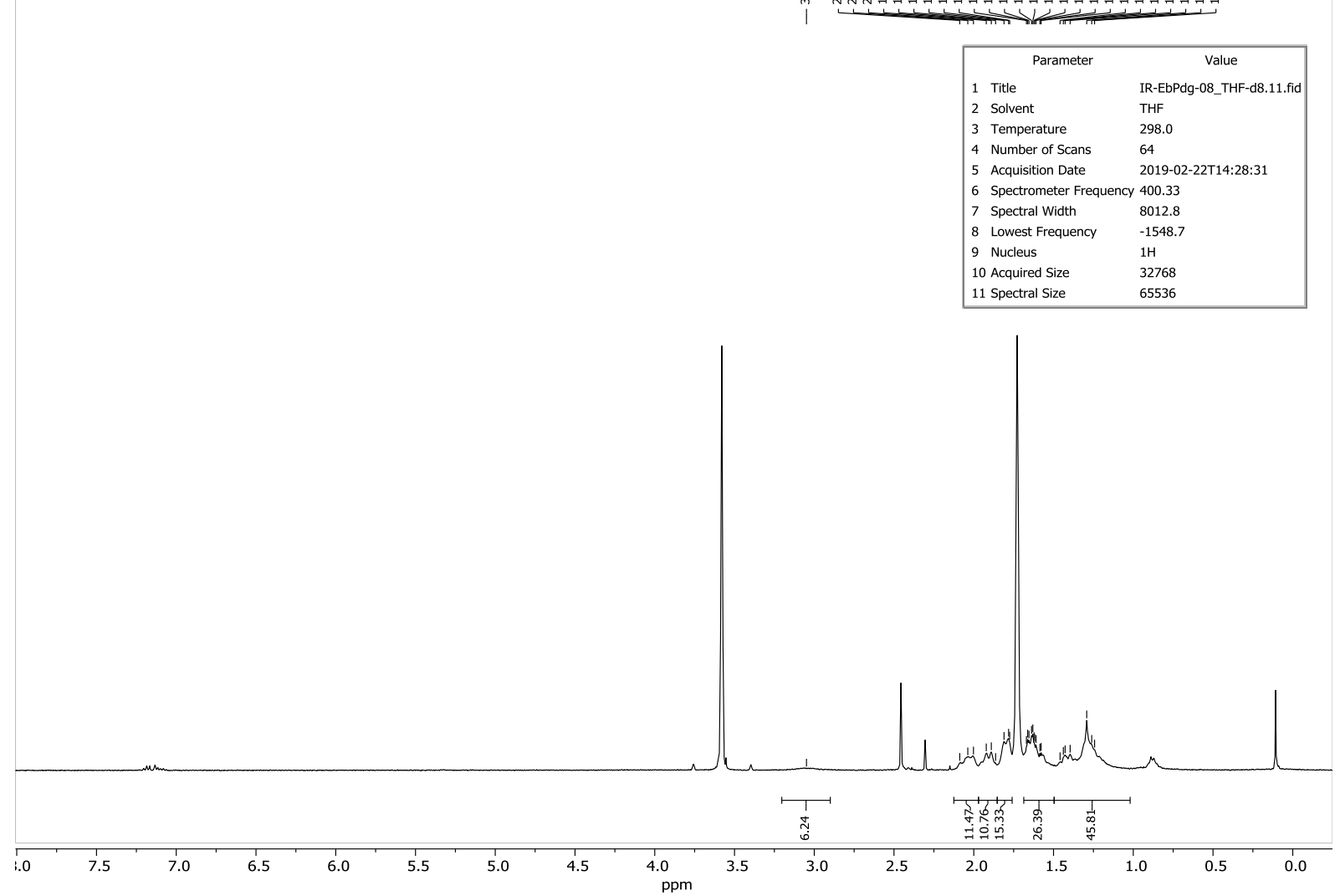

Figure S2b. ${ }^{1} \mathrm{H}$ NMR spectrum of $\mathbf{L 1}_{2} \cdot \mathbf{P d}$. 


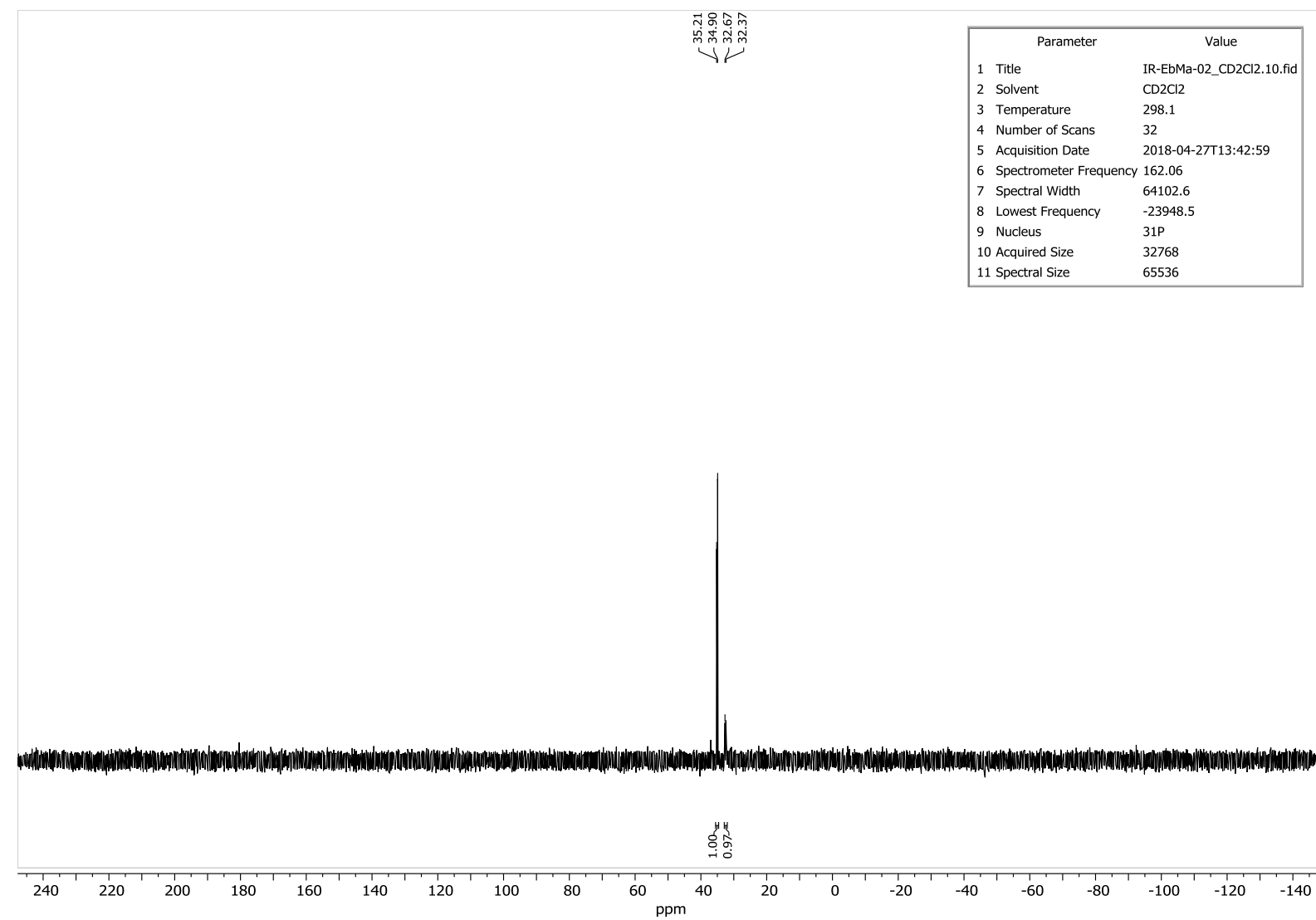

Figure S3a. ${ }^{31} \mathrm{P}\left\{{ }^{1} \mathrm{H}\right\}$ NMR spectrum of $\mathbf{2}$.

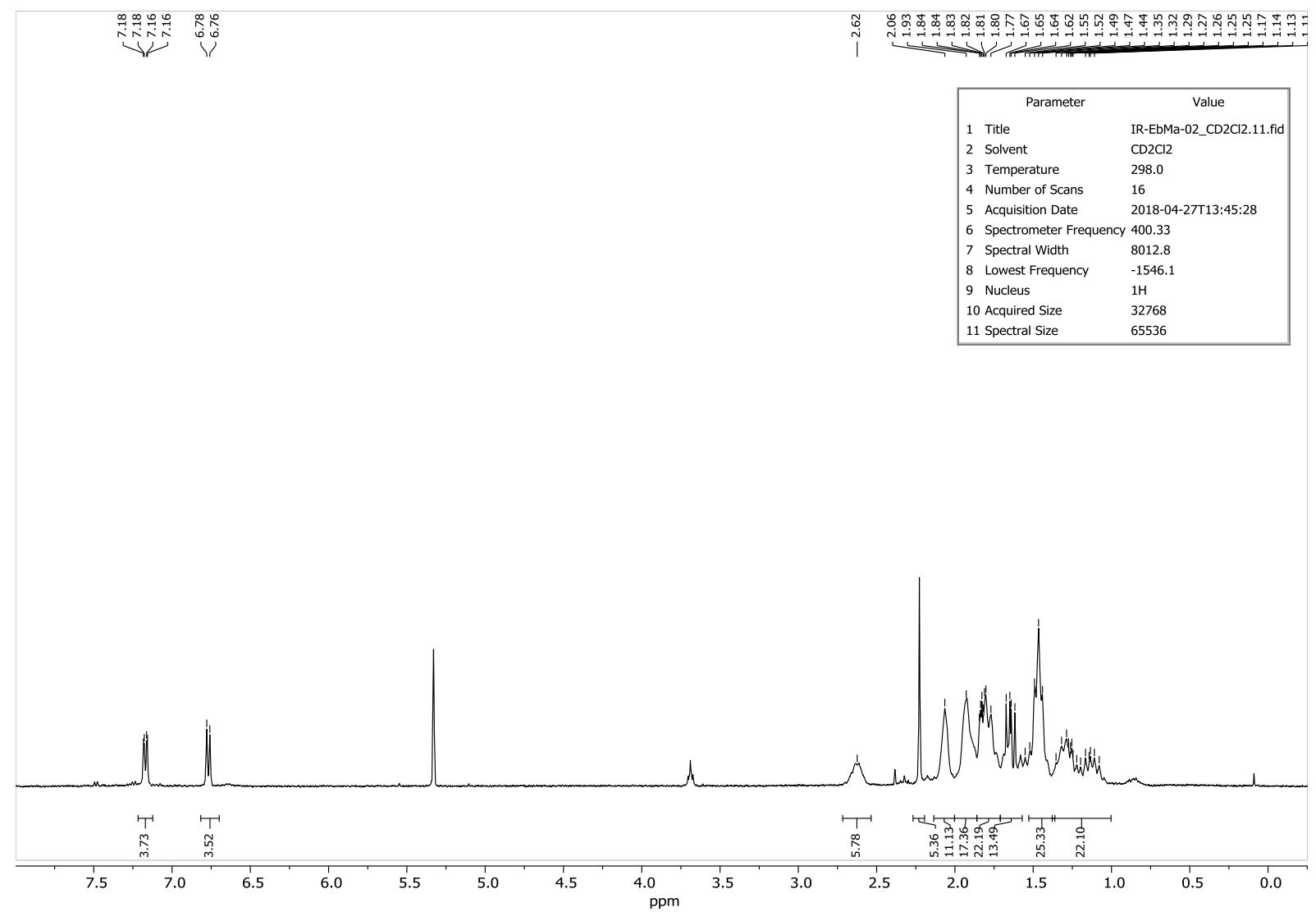

Figure S3b. ${ }^{1} \mathrm{H}$ NMR spectrum of $2 \mathbf{2}$. 


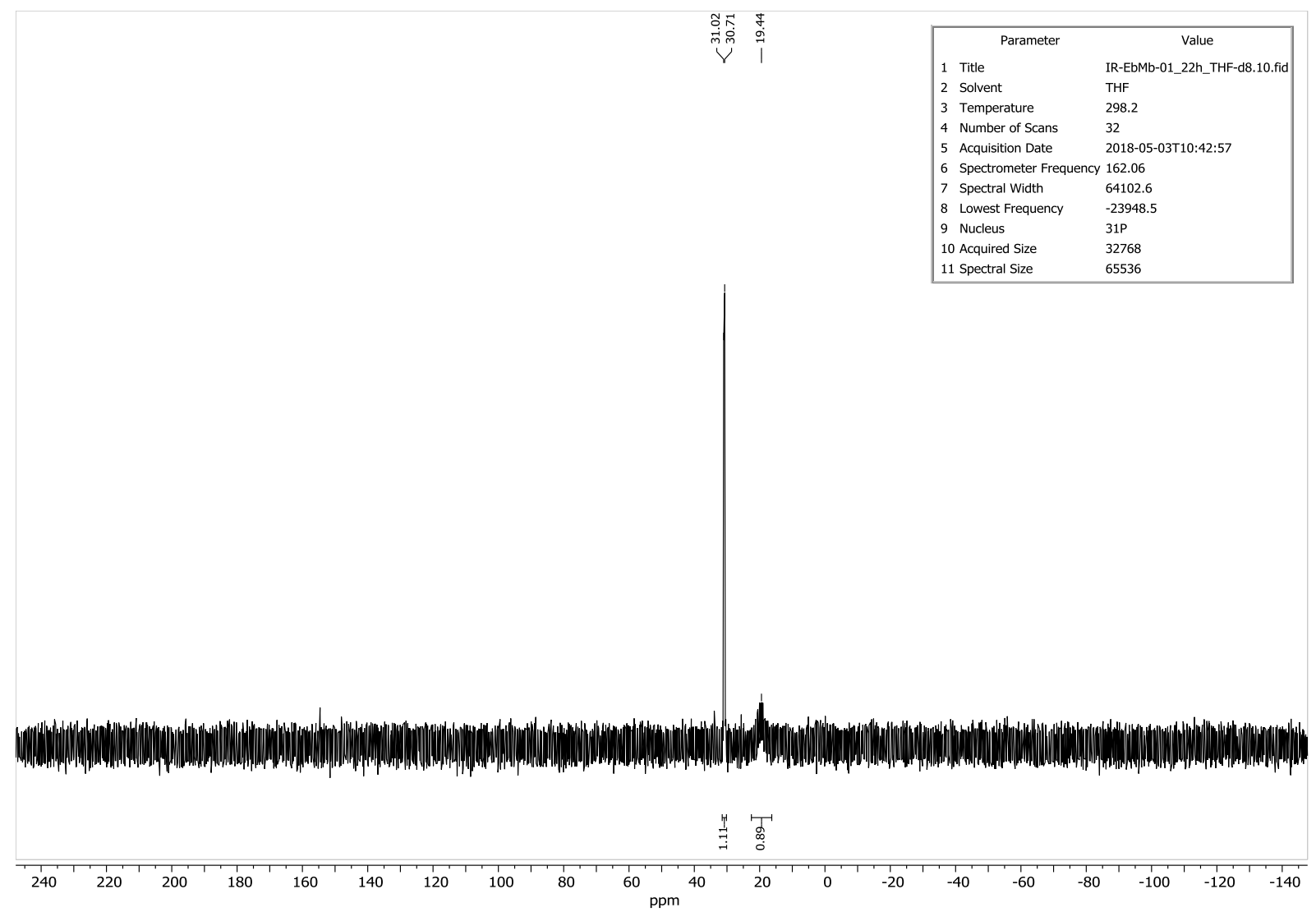

Figure S4a. ${ }^{31} \mathrm{P}\left\{{ }^{1} \mathrm{H}\right\}$ NMR spectrum of $\mathbf{3}^{\text {Tol }}$ with a large excess of diethylamine.

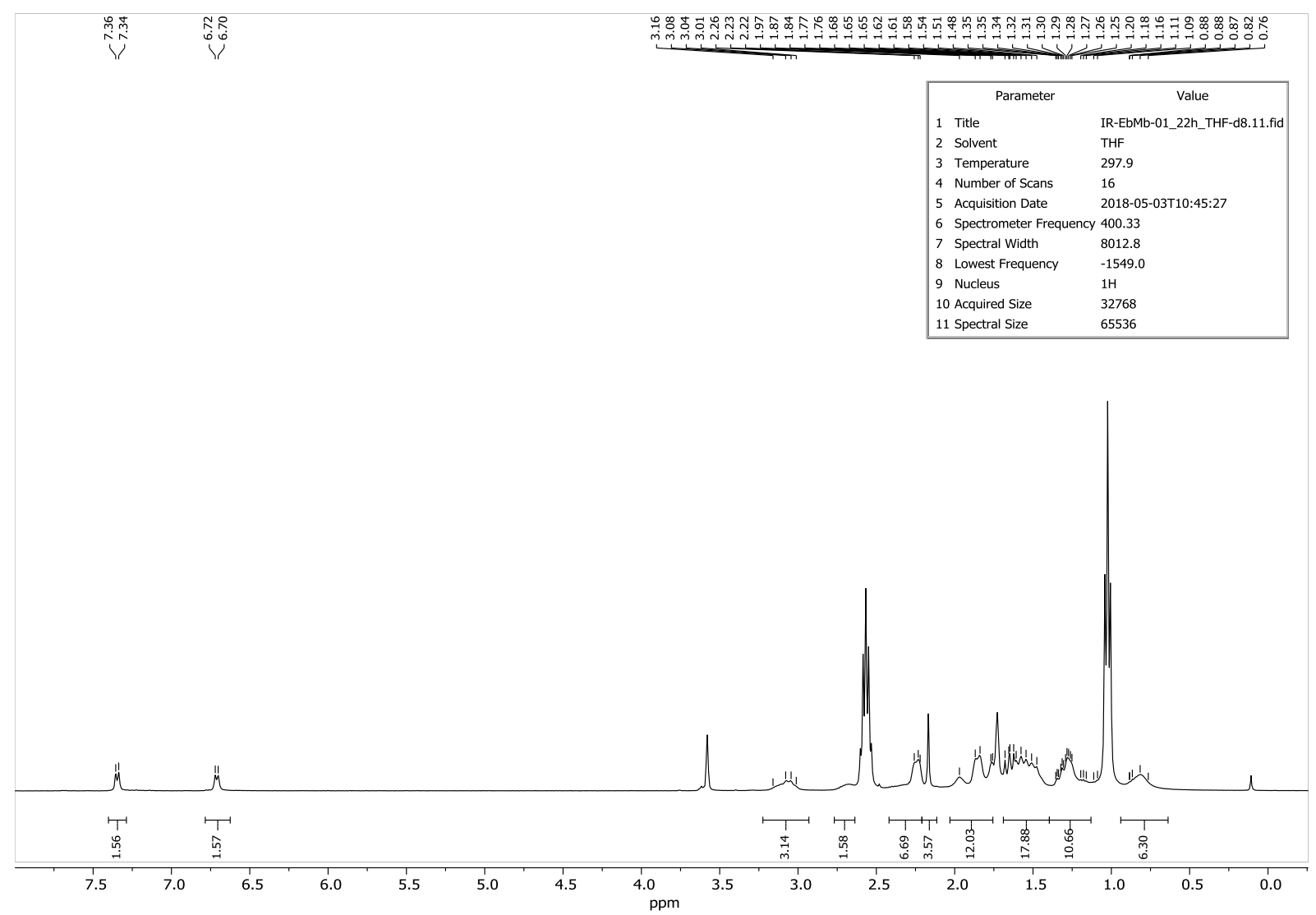

Figure S4b. ${ }^{1} \mathrm{H}$ NMR spectrum of $3^{\text {Tol }}$ with a large excess of diethylamine. 


\section{Catalytic and Kinetic studies}

\subsection{Procedure for the BHA with L1, L2 and L3 (Table 1)}

A $5 \mathrm{ml}$ vial with septum cap was charged with $168.3 \mathrm{mg}(1.5 \mathrm{mmol}, 1.5 \mathrm{eq}$.) of potassium tert-butoxide. which was then dissolved in $3 \mathrm{ml}$ of dry tetrahydrofurane. Subsequently, $0.05 \mathrm{ml}(40 \mathrm{mg}, 0.2 \mathrm{mmol}, 0.2$ eq.) tetradecane (GC standard), $1.0 \mathrm{mmol}$ (1.0 eq.) aryl chloride and $0.10 \mathrm{ml}$ ( $85 \mathrm{mg}, 1.0 \mathrm{mmol}, 1.0$ eq.) piperidine were added. Another $5 \mathrm{ml}$ vial with septum cap was charged with $0.05 \mathrm{mmol}(0.005 \mathrm{eq}$.) of the ligand and $3.4 \mathrm{mg}\left(0.025 \mathrm{mmol}, 0.0025 \mathrm{eq}\right.$.) of $\mathrm{Pd}_{2}(\mathrm{dba})_{3} \cdot \mathrm{dba}$. Both compounds were suspended in $0.5 \mathrm{ml}$ of dry THF and stirred for 30 minutes. The catalyst solution was then added with a syringe to the reaction solution to start the catalytic reaction.

For reaction monitoring, a tiny amount of the reaction solution was taken out of the reaction mixture and 1 drop of the solution was diluted in $5 \mathrm{ml}$ of ethyl acetate. The mixture was filtrated through a short plug of silica and the product ratio was determined via GC-FID by comparison of the product and standard peaks via integration. The obtained results are provided in Table 1.

\subsection{Procedure and data of the kinetic studies with keYPhos, L1}

\section{Procedure:}

A Schlenk flask was charged with $1.68 \mathrm{~g}$ of dry potassium tert-butoxide, which was dissolved in $23.3 \mathrm{ml}$ of dry tetrahydrofurane. $0.52 \mathrm{ml}(0.40 \mathrm{~g}, 2 \mathrm{mmol}, 0.2 \mathrm{eq}$ ) of tetradecane (GC standard), $1.18 \mathrm{ml}(1.26$ $\mathrm{g}, 10 \mathrm{mmol}, 1$ eq.) 4-chlorotoluene and $0.99 \mathrm{ml}(0.85 \mathrm{~g}, 10 \mathrm{mmol}, 1 \mathrm{eq}$.) piperidine were added to this mixture one after the other. In the different experiments outlined below, the amount of 4-chlorotoluene (experiment II) and piperidine (experiment III) was doubled. In this case, the amount of dry THF was decreased to maintain a constant volume of the reaction solution.

A $5 \mathrm{ml}$ vial with a septum cap was charged with $25.2 \mathrm{mg}(0.05 \mathrm{mmol}, 0.005$ eq. $)$ of ligand $\mathbf{L} 1$ and $34.4 \mathrm{mg}$ ( $0.05 \mathrm{mmol}, 0.005$ eq.) of $\mathrm{Pd}_{2}(\mathrm{dba})_{3} \cdot \mathrm{dba}$, which was dissolved in $4 \mathrm{ml}$ of dry THF and stirred for 30 minutes. After this period of time, the dark red solution was added to the reaction flask to start the catalytic reaction.

Every 2 minutes, one drop of reaction solution was dissolved in $5 \mathrm{ml}$ of ethyl acetate and filtered through a short plug of silica. The product ratio was determined via GC-FID, by comparison of the product and standard peaks via integration.

This procedure was used for the different excess experiments with different $[\mathrm{ArCl}] / \mathrm{Cl}$ :

Table S1. Amounts of reagents used in the different excess experiments. Total reaction volume: $30 \mathrm{~mL}$.

\begin{tabular}{|l|c|c|c|c|}
\hline & Mol (ArCl) & [ArCl] & Mol (amine) & [amine] \\
\hline Experiment I: (no excess) & $9.97 \mathrm{mmol}$ & $0.332 \mathrm{M}$ & $9.97 \mathrm{mmol}$ & $0.332 \mathrm{M}$ \\
\hline Experiment II: (2eq ArCl) & $19.9 \mathrm{mmol}$ & $0.663 \mathrm{M}$ & $9.97 \mathrm{mmol}$ & $0.332 \mathrm{M}$ \\
\hline Experiment III: (2eq Amine) & $9.97 \mathrm{mmol}$ & $0.332 \mathrm{M}$ & $19.9 \mathrm{mmol}$ & $0.663 \mathrm{M}$ \\
\hline
\end{tabular}


Table S2. Results of the different excess experiments

\section{Mol(ArCl) during the experiments I-III}

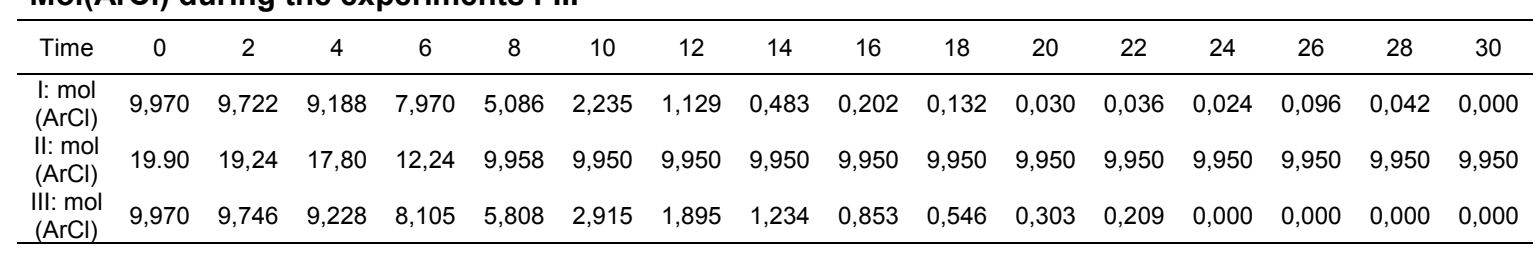

\section{Concentrations of $\mathrm{ArCl}$ during the reaction progress of the experiments I-III}

\begin{tabular}{|c|c|c|c|c|c|c|c|c|c|c|c|c|c|c|c|c|}
\hline Time & 0 & 2 & 4 & 6 & 8 & 10 & 12 & 14 & 16 & 18 & 20 & 22 & 24 & 26 & 28 & 30 \\
\hline $\begin{array}{c}\text { I: M } \\
(\mathrm{ArCl})\end{array}$ & 0,332 & 0,324 & 0,306 & 0,266 & 0,170 & 0,075 & 0,038 & 0,016 & 0,007 & 0,004 & 0,001 & 0,001 & 0,001 & 0,003 & 0,001 & 0,000 \\
\hline $\begin{array}{l}\text { II: M } \\
\text { (ArCl) }\end{array}$ & 0,663 & 0,641 & 0,593 & 0,408 & 0,335 & 0,332 & 0,332 & 0,332 & 0,332 & 0,332 & 0,332 & 0,332 & 0,332 & 0,332 & 0,332 & 0,332 \\
\hline $\begin{array}{l}\text { III: M } \\
\text { (ArCl) }\end{array}$ & 0,332 & 0,325 & 0,308 & 0,270 & 0,194 & 0,097 & 0,063 & 0,041 & 0,028 & 0,018 & 0,010 & 0,007 & 0,000 & 0,000 & 0,000 & 0,000 \\
\hline
\end{tabular}

\section{Reaction rate oft he experiments I-III}

\begin{tabular}{|c|c|c|c|c|c|c|c|c|c|c|c|c|c|c|c|c|}
\hline $\begin{array}{l}\text { Time } \\
{[\mathrm{min}]}\end{array}$ & 0 & 2 & 4 & 6 & 8 & 10 & 12 & 14 & 16 & 18 & 20 & 22 & 24 & 26 & 28 & 30 \\
\hline $\begin{array}{c}\text { I: rate } \\
\text { [mol/L. } \\
\mathrm{min}]\end{array}$ & 0 & 0,004 & 0,009 & 0,020 & 0,048 & 0,048 & 0,018 & 0,011 & 0,005 & 0,001 & 0,002 & 0,000 & 0,000 & 0,001 & 0,001 & 0,001 \\
\hline $\begin{array}{c}\text { III: } \\
\text { rate } \\
{[\mathrm{mol} / \mathrm{L} \cdot} \\
\mathrm{min}]\end{array}$ & 0 & 0,004 & 0,009 & 0,019 & 0,038 & 0,048 & 0,017 & 0,011 & 0,006 & 0,005 & 0,004 & 0,002 & 0,003 & 0,000 & 0,000 & 0,000 \\
\hline
\end{tabular}

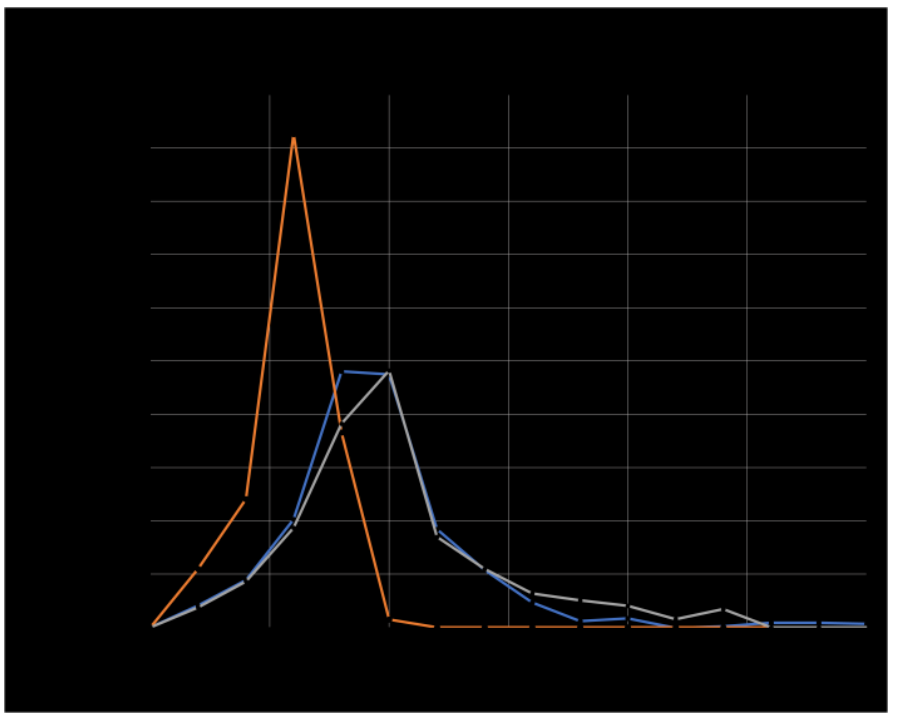

Figure S5. Plot of the different excess experiments: reaction rate versus time. Blue (I: no excess), orange (II, excess $\mathrm{ArCl})$, grey (III: excess amine). 


\subsection{Variable time normalization analysis}

The data obtained from the GC analysis studies of the reaction progress were transformed into [ArCl] and [amine] during the reaction (Table S2). These concentrations were used in the form provided by Burés and coworkers in: Nielsen, C. D.-T.; Burés, J. Visual kinetic analysis, Chem. Sci. 2019, 101, 348. The order was changed until graphical profile of both experiments gave an exact overlay.

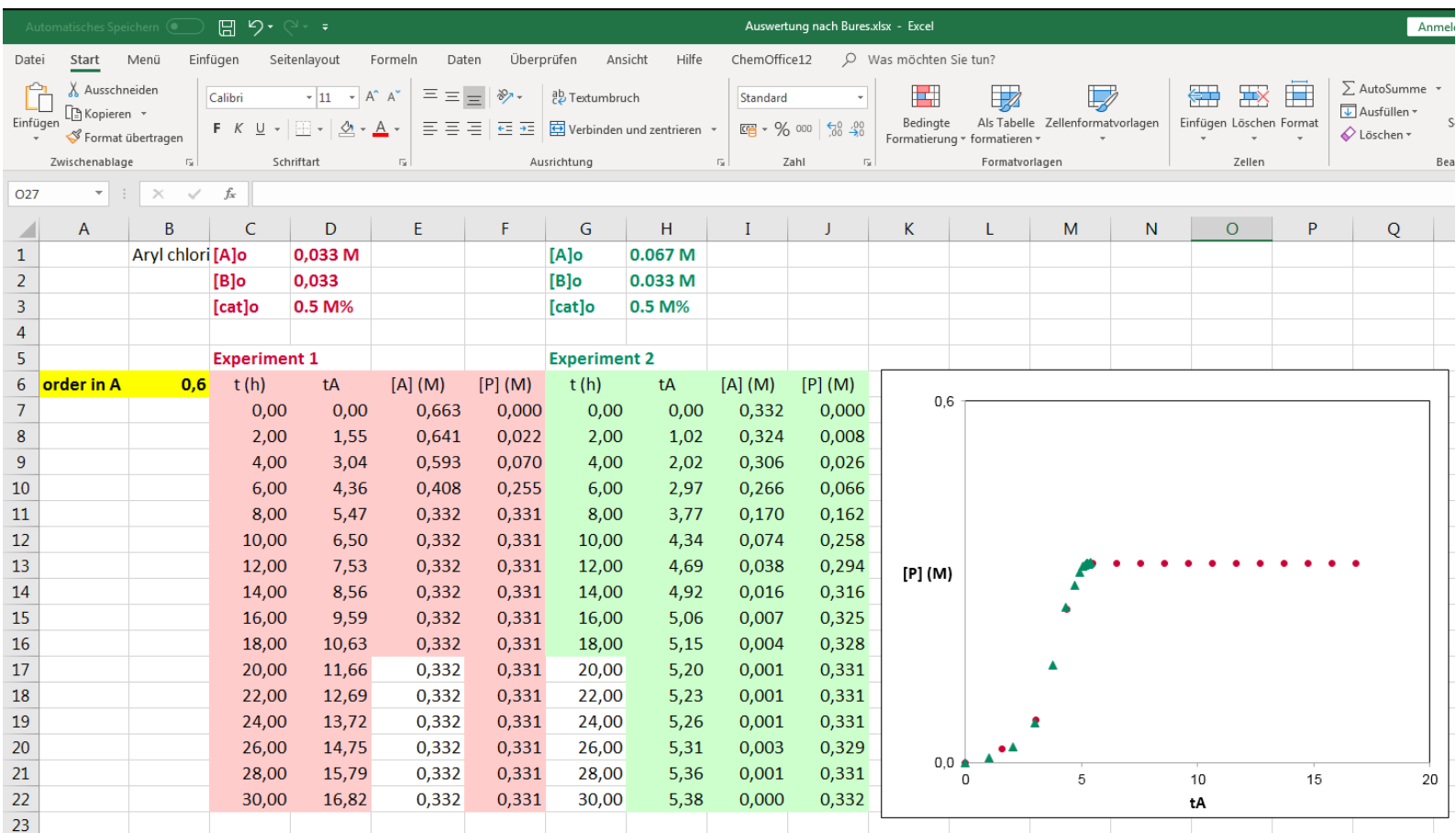

Figure S6. Variable time normalization analysis with the data from experiment I (no excess) and experiment II (excess $\mathrm{ArCl}$ ). Analysis gives an overlay of the two reaction profiles with a reaction order of 0.6 in $\mathrm{ArCl}$.

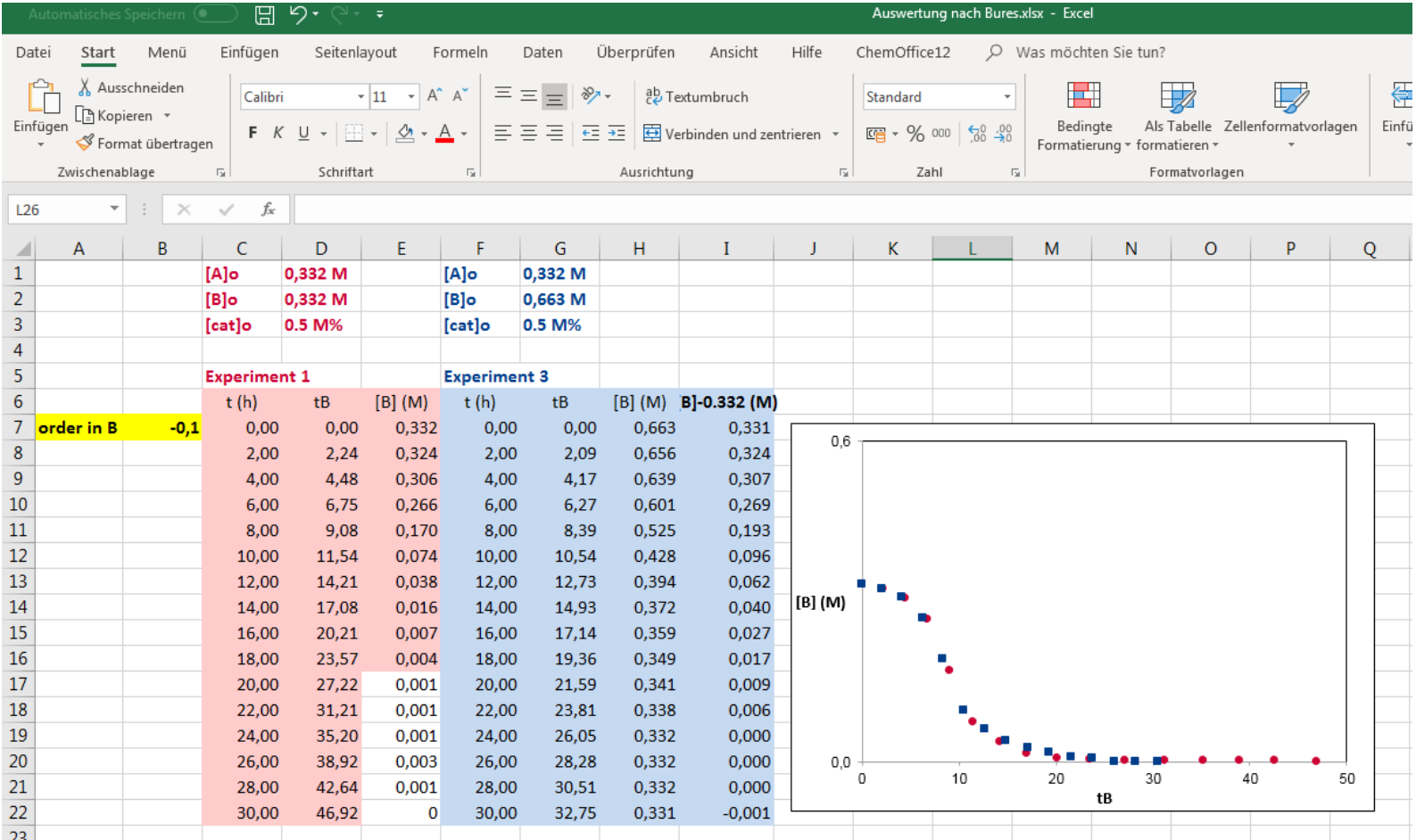

Figure S7. Variable time normalization analysis with the data from experiment I (no excess) and experiment III (excess amine). Analysis gives an overlay of the two reaction profiles with a reaction order of -0.1 in amine, which we interpret as a zeroth-order dependency. 


\section{Crystal Structure Determination}

\subsection{General information}

Details on the structure solution: $\mathbf{2}_{\mathbf{2}}{ }^{\text {Tol }}$ contained three THF molecules, two of them were disordered. One is located on an inversion center, the other one is disordered over two positions with an occupancy of $0.65: 0.35$. All THF molecules were modelled by using SAME, RIGU and SIMU restrains.

Table S3. Data collection and structure refinement details for the compounds $\mathbf{L} \mathbf{1}_{2} \cdot \mathbf{P d}, \mathbf{2}_{2}{ }^{\mathrm{Tol}}$ and $\mathbf{3}^{\mathrm{Tol}}$.

\begin{tabular}{|c|c|c|c|}
\hline Compound & $\mathrm{L}_{2} \cdot \mathrm{Pd}$ & $\mathbf{2}_{2}^{\mathrm{Tol}}$ & $3^{\text {Tol }}$ \\
\hline CCDC No. & 1913509 & 1913510 & 1913511 \\
\hline Formula & $\mathrm{C}_{64} \mathrm{H}_{116} \mathrm{P}_{4} \mathrm{Pd}$ & $\mathrm{C}_{49} \mathrm{H}_{85} \mathrm{ClO}_{2.5} \mathrm{P}_{2} \mathrm{Pd}$ & $\mathrm{C}_{43} \mathrm{H}_{76} \mathrm{CINP}_{2} \mathrm{Pd}$ \\
\hline Formula weight $\left[\mathrm{g} \cdot \mathrm{mol}^{-1}\right]$ & 1115.84 & 917.95 & 810.83 \\
\hline Temperature $[\mathrm{K}]$ & $100.4(6)$ & $100.0(3)$ & $99.90(14)$ \\
\hline Wave length $[\AA]]$ & 1.54184 & 1.54184 & 1.54184 \\
\hline Crystal system & Triclinic & Triclinic & Triclinic \\
\hline Space group & $\mathrm{P} \overline{1}$ & $\mathrm{P} \overline{1}$ & $\mathrm{P} \overline{1}$ \\
\hline $\mathrm{a}[\AA]$ & $11.6891(3)$ & $11.8057(3)$ & $11.3247(6)$ \\
\hline $\mathrm{b}[\AA]]$ & $12.0707(5)$ & $15.6134(3)$ & $12.9036(7)$ \\
\hline$c[\AA]$ & $23.6505(5)$ & $15.6605(4)$ & $15.5260(9)$ \\
\hline$\alpha\left[^{\circ}\right]$ & $80.935(2)$ & $116.403(2)$ & $86.814(4)$ \\
\hline$\beta\left[{ }^{\circ}\right]$ & $76.320(2)$ & $99.017(2)$ & $87.399(4)$ \\
\hline $\mathrm{Y}\left[{ }^{\circ}\right]$ & $70.620(3)$ & $106.380(2)$ & $68.191(5)$ \\
\hline Volume $\left[\AA^{3}\right]$ & $3047.18(17)$ & $2343.10(11)$ & $2102.4(2)$ \\
\hline Z & 2 & 2 & 2 \\
\hline Calc. density $\left[\mathrm{Mg} \cdot \mathrm{m}^{-3}\right]$ & 1.216 & 1.301 & 1.281 \\
\hline$\mu\left[\mathrm{mm}^{-1}\right]$ & 3.712 & 4.645 & 5.069 \\
\hline$F(000)$ & 1212 & 984 & 868 \\
\hline Crystal dimensions [mm] & $\begin{array}{l}0.146 \times 0.052 \times \\
0.031\end{array}$ & $\begin{array}{l}0.143 \times 0.078 \times \\
0.027\end{array}$ & $\begin{array}{l}0.123 \times 0.081 \times \\
0.024\end{array}$ \\
\hline Theta range $\left[{ }^{\circ}\right]$ & 3.862 to 68.251 & 3.336 to 70.073 & 3.693 to $70.060^{\circ}$ \\
\hline \multirow[t]{3}{*}{ Index ranges } & $-14 \leq \mathrm{h} \leq 14$ & $-14 \leq \mathrm{h} \leq 14$ & $-13 \leq h \leq 13$ \\
\hline & $-14 \leq k \leq 13$ & $-19 \leq k \leq 19$ & $-15 \leq k \leq 15$ \\
\hline & $-28 \leq 1 \leq 28$ & $-19 \leq 1 \leq 19$ & $-17 \leq \mid \leq 18$ \\
\hline Reflections collected & 44292 & 34788 & 30992 \\
\hline Independent reflections & $11136\left[R_{\text {int }}=0.0447\right]$ & $8891\left[R_{\text {int }}=0.0338\right]$ & $7969\left[R_{\text {int }}=0.0420\right]$ \\
\hline $\begin{array}{l}\text { Data/Restraints/Paramet } \\
\text { er }\end{array}$ & $11136 / 0 / 624$ & 8891 / 256 / 572 & 7969 / 0 / 440 \\
\hline Goodness-of-fit on $F^{2}$ & 1.023 & 1.032 & 1.034 \\
\hline $\begin{array}{l}\text { Final } R \text { indices } \\
{[\mid>2 \text { sigma }(I)]}\end{array}$ & $\begin{array}{l}\mathrm{R} 1=0.0323, w R 2= \\
0.0760\end{array}$ & $\begin{array}{l}\mathrm{R} 1=0.0273, w R 2= \\
0.0686\end{array}$ & $\begin{array}{l}\mathrm{R} 1=0.0283, \mathrm{wR} 2= \\
0.0712\end{array}$ \\
\hline$R$ indices (all data) & $\begin{array}{l}\mathrm{R} 1=0.0444, w R 2= \\
0.0835\end{array}$ & $\begin{array}{l}\mathrm{R} 1=0.0301, \mathrm{wR} 2= \\
0.0707\end{array}$ & $\begin{array}{l}\mathrm{R} 1=0.0341, \mathrm{wR} 2= \\
0.0748\end{array}$ \\
\hline $\begin{array}{l}\text { Largest diff. peak and } \\
\text { hole }\left[\mathrm{e} \cdot \AA^{-3}\right]\end{array}$ & 0.743 and -0.448 & 0.882 and -0.554 & 0.751 and -0.761 \\
\hline
\end{tabular}




\subsection{Crystal structures}

\subsubsection{Crystal Structure Determination of $L 1_{2} \cdot P d$}

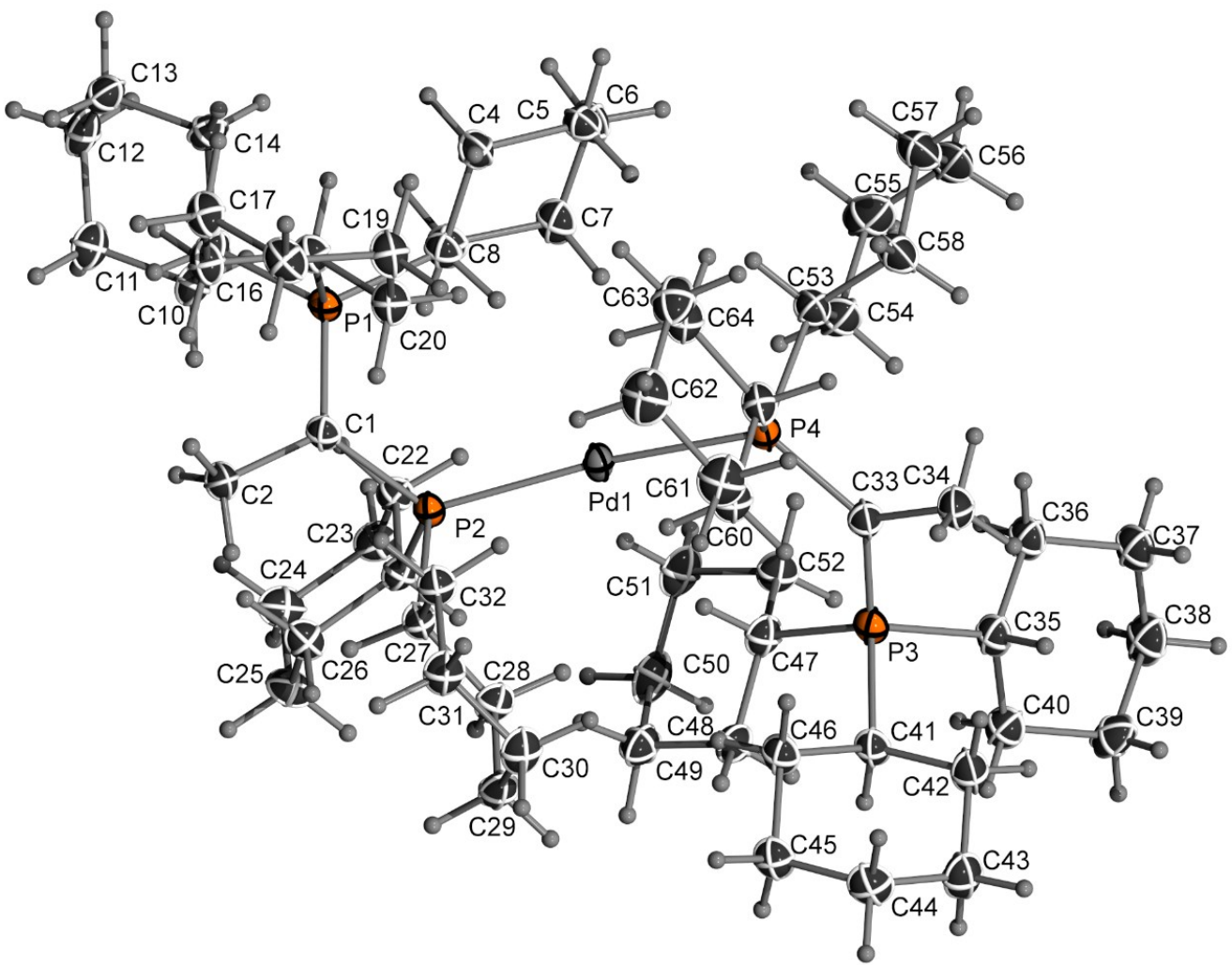

Figure S8. ORTEP Plot of $\mathbf{L 1}_{2} \cdot \mathbf{P d}$. Ellipsoids are drawn at the $50 \%$ probability level.

Table S4. Atomic coordinates $\left(\times 10^{4}\right)$ and equivalent isotropic displacement parameters $\left(\AA^{2} \times 10^{3}\right)$ for $\mathbf{L} 1_{2} \cdot \mathbf{P d}$. $\mathrm{U}(\mathrm{eq})$ is defined as one third of the trace of the orthogonalized $\mathrm{U}^{\mathrm{ij}}$ tensor.

\begin{tabular}{ccccc} 
& $\mathrm{x}$ & $\mathrm{y}$ & $\mathrm{z}$ & $\mathrm{U}(\mathrm{eq})$ \\
\hline \hline $\mathrm{Pd}(1)$ & $3188(1)$ & $5257(1)$ & $2563(1)$ & $20(1)$ \\
$\mathrm{P}(1)$ & $3042(1)$ & $3312(1)$ & $1261(1)$ & $17(1)$ \\
$\mathrm{P}(2)$ & $2890(1)$ & $5748(1)$ & $1601(1)$ & $17(1)$ \\
$\mathrm{P}(3)$ & $3401(1)$ & $7241(1)$ & $3841(1)$ & $21(1)$ \\
$\mathrm{P}(4)$ & $3987(1)$ & $4762(1)$ & $3412(1)$ & $21(1)$ \\
$\mathrm{C}(1)$ & $3030(2)$ & $4744(2)$ & $1091(1)$ & $19(1)$ \\
$\mathrm{C}(2)$ & $3225(2)$ & $5118(2)$ & $433(1)$ & $24(1)$ \\
$\mathrm{C}(3)$ & $2604(2)$ & $2981(2)$ & $2049(1)$ & $21(1)$ \\
$\mathrm{C}(4)$ & $3107(2)$ & $1692(2)$ & $2289(1)$ & $25(1)$ \\
$\mathrm{C}(5)$ & $2802(2)$ & $1614(2)$ & $2959(1)$ & $27(1)$ \\
$\mathrm{C}(6)$ & $1424(2)$ & $2130(3)$ & $3202(1)$ & $29(1)$ \\
$\mathrm{C}(7)$ & $937(2)$ & $3396(2)$ & $2949(1)$ & $28(1)$ \\
$\mathrm{C}(8)$ & $1201(2)$ & $3442(2)$ & $2281(1)$ & $23(1)$ \\
$\mathrm{C}(9)$ & $2058(2)$ & $2956(2)$ & $849(1)$ & $21(1)$ \\
$\mathrm{C}(10)$ & $753(2)$ & $3843(2)$ & $872(1)$ & $24(1)$ \\
$\mathrm{C}(11)$ & $122(2)$ & $3566(2)$ & $435(1)$ & $29(1)$ \\
$\mathrm{C}(12)$ & $55(2)$ & $2309(3)$ & $550(1)$ & $31(1)$
\end{tabular}




\begin{tabular}{|c|c|c|c|c|}
\hline$C(13)$ & $1346(2)$ & $1422(2)$ & $542(1)$ & $29(1)$ \\
\hline$C(14)$ & 1988(2) & $1687(2)$ & $976(1)$ & $24(1)$ \\
\hline$C(15)$ & $4554(2)$ & $2156(2)$ & $1043(1)$ & $21(1)$ \\
\hline$C(16)$ & $4984(2)$ & 1998(2) & $384(1)$ & $24(1)$ \\
\hline$C(17)$ & $6201(2)$ & $991(2)$ & $269(1)$ & $26(1)$ \\
\hline$C(18)$ & $7206(2)$ & $1189(2)$ & $514(1)$ & $27(1)$ \\
\hline$C(19)$ & $6778(2)$ & $1372(2)$ & $1165(1)$ & $27(1)$ \\
\hline$C(20)$ & $5566(2)$ & $2381(2)$ & $1277(1)$ & $25(1)$ \\
\hline$C(21)$ & $1418(2)$ & $6988(2)$ & $1560(1)$ & $19(1)$ \\
\hline$C(22)$ & $295(2)$ & $6539(2)$ & $1795(1)$ & $20(1)$ \\
\hline$C(23)$ & $-940(2)$ & $7521(2)$ & $1802(1)$ & $25(1)$ \\
\hline$C(24)$ & $-1008(2)$ & $8195(2)$ & $1202(1)$ & $28(1)$ \\
\hline$C(25)$ & $80(2)$ & $8690(2)$ & 992(1) & $28(1)$ \\
\hline$C(26)$ & $1308(2)$ & $7697(2)$ & $965(1)$ & $23(1)$ \\
\hline$C(27)$ & $4053(2)$ & $6533(2)$ & $1240(1)$ & $20(1)$ \\
\hline$C(28)$ & $3888(2)$ & $7575(2)$ & $1576(1)$ & $23(1)$ \\
\hline$C(29)$ & $4911(2)$ & $8161(2)$ & $1342(1)$ & $26(1)$ \\
\hline$C(30)$ & $6183(2)$ & $7259(3)$ & $1345(1)$ & $29(1)$ \\
\hline$C(31)$ & $6379(2)$ & $6245(2)$ & $987(1)$ & $26(1)$ \\
\hline$C(32)$ & $5360(2)$ & $5654(2)$ & $1200(1)$ & $22(1)$ \\
\hline$C(33)$ & $4148(2)$ & $5762(2)$ & $3842(1)$ & $24(1)$ \\
\hline$C(34)$ & $5138(2)$ & $5331(2)$ & $4223(1)$ & $32(1)$ \\
\hline$C(35)$ & $2780(2)$ & $7693(2)$ & $4600(1)$ & $25(1)$ \\
\hline$C(36)$ & $2079(2)$ & $6908(3)$ & $5008(1)$ & $29(1)$ \\
\hline$C(37)$ & $1779(2)$ & $7229(3)$ & $5640(1)$ & $33(1)$ \\
\hline$C(38)$ & $1061(3)$ & $8532(3)$ & $5686(1)$ & $37(1)$ \\
\hline$C(39)$ & $1758(3)$ & $9315(3)$ & $5280(1)$ & $34(1)$ \\
\hline$C(40)$ & $2055(2)$ & $9009(2)$ & $4645(1)$ & $29(1)$ \\
\hline$C(41)$ & $4396(2)$ & $8213(2)$ & $3555(1)$ & $23(1)$ \\
\hline$C(42)$ & $5289(2)$ & $8223(3)$ & $3940(1)$ & $31(1)$ \\
\hline$C(43)$ & $5979(2)$ & $9118(3)$ & $3670(1)$ & $33(1)$ \\
\hline$C(44)$ & $6679(2)$ & $8865(3)$ & $3049(1)$ & $30(1)$ \\
\hline$C(45)$ & $5815(2)$ & $8822(2)$ & $2665(1)$ & $26(1)$ \\
\hline$C(46)$ & $5124(2)$ & $7931(2)$ & 2933(1) & $25(1)$ \\
\hline$C(47)$ & $2185(2)$ & $7720(2)$ & $3399(1)$ & $24(1)$ \\
\hline$C(48)$ & 1902(2) & $9006(2)$ & $3137(1)$ & $27(1)$ \\
\hline$C(49)$ & $944(2)$ & $9307(2)$ & $2747(1)$ & $29(1)$ \\
\hline$C(50)$ & $-231(2)$ & $9014(3)$ & $3063(1)$ & $34(1)$ \\
\hline$C(51)$ & $68(2)$ & $7741(3)$ & $3316(1)$ & $32(1)$ \\
\hline$C(52)$ & $974(2)$ & $7480(3)$ & $3731(1)$ & $28(1)$ \\
\hline$C(53)$ & $3315(2)$ & $3673(2)$ & $3932(1)$ & $26(1)$ \\
\hline$C(54)$ & $2005(2)$ & $4298(3)$ & $4250(1)$ & $34(1)$ \\
\hline$C(55)$ & 1358(3) & $3450(3)$ & $4642(1)$ & $41(1)$ \\
\hline$C(56)$ & $2156(3)$ & $2619(3)$ & $5056(1)$ & $47(1)$ \\
\hline$C(57)$ & $3416(3)$ & 1978(3) & $4715(1)$ & $43(1)$ \\
\hline$C(58)$ & $4076(3)$ & $2856(3)$ & $4372(1)$ & $36(1)$ \\
\hline$C(59)$ & $5630(2)$ & $3815(2)$ & $3192(1)$ & $24(1)$ \\
\hline$C(60)$ & $6405(2)$ & $4564(2)$ & $2807(1)$ & $28(1)$ \\
\hline$C(61)$ & $7742(2)$ & $3823(3)$ & $2588(1)$ & $36(1)$ \\
\hline$C(62)$ & $7794(2)$ & $2805(3)$ & $2265(1)$ & $40(1)$ \\
\hline
\end{tabular}




\begin{tabular}{lllll}
$\mathrm{C}(63)$ & $7041(2)$ & $2050(3)$ & $2646(1)$ & $35(1)$ \\
$\mathrm{C}(64)$ & $5702(2)$ & $2780(3)$ & $2871(1)$ & $30(1)$ \\
\hline
\end{tabular}

Table S5. Anisotropic displacement parameters $\left(\AA^{2} \times 10^{3}\right)$ for $\mathbf{L} \mathbf{1}_{2} \cdot \mathbf{P d}$. The anisotropic displacement factor exponent takes the form: $-2 \pi^{2}\left[h^{2} a^{2}{ }^{2} U^{11}+\ldots+2 h k a \cdot b \cdot U^{12}\right]$.

\begin{tabular}{|c|c|c|c|c|c|c|}
\hline & $U^{11}$ & $U^{22}$ & $\mathrm{U}^{33}$ & $\mathrm{U}^{23}$ & $U^{13}$ & $U^{12}$ \\
\hline $\mathrm{Pd}(1)$ & 19(1) & $26(1)$ & $15(1)$ & $0(1)$ & $-3(1)$ & $-9(1)$ \\
\hline$P(1)$ & $15(1)$ & $20(1)$ & $16(1)$ & $-1(1)$ & $-1(1)$ & $-7(1)$ \\
\hline $\mathrm{P}(2)$ & 15(1) & $22(1)$ & 15(1) & $-1(1)$ & $-3(1)$ & $-7(1)$ \\
\hline $\mathrm{P}(3)$ & $20(1)$ & $27(1)$ & $18(1)$ & $-2(1)$ & $-1(1)$ & $-11(1)$ \\
\hline$P(4)$ & $23(1)$ & $26(1)$ & $16(1)$ & $0(1)$ & $-4(1)$ & $-10(1)$ \\
\hline$C(1)$ & 18(1) & $23(1)$ & $17(1)$ & $-1(1)$ & $-2(1)$ & $-9(1)$ \\
\hline$C(2)$ & $31(1)$ & $25(1)$ & $19(1)$ & $-2(1)$ & $-3(1)$ & $-10(1)$ \\
\hline$C(3)$ & $20(1)$ & $25(1)$ & $18(1)$ & $-1(1)$ & $0(1)$ & $-10(1)$ \\
\hline$C(4)$ & $28(1)$ & $25(1)$ & $22(1)$ & $0(1)$ & $-3(1)$ & $-10(1)$ \\
\hline$C(5)$ & $31(1)$ & $29(1)$ & $22(1)$ & $4(1)$ & $-6(1)$ & $-15(1)$ \\
\hline$C(6)$ & $33(1)$ & $38(2)$ & $18(1)$ & $-1(1)$ & 1(1) & $-18(1)$ \\
\hline$C(7)$ & $24(1)$ & $38(2)$ & $22(1)$ & $-5(1)$ & $3(1)$ & $-14(1)$ \\
\hline$C(8)$ & $20(1)$ & $24(1)$ & $23(1)$ & $-2(1)$ & $0(1)$ & $-8(1)$ \\
\hline$C(9)$ & 19(1) & $24(1)$ & 21(1) & $-4(1)$ & $-3(1)$ & $-9(1)$ \\
\hline$C(10)$ & $20(1)$ & $29(1)$ & $25(1)$ & $-6(1)$ & $-4(1)$ & $-7(1)$ \\
\hline$C(11)$ & 23(1) & $36(2)$ & $30(1)$ & $-7(1)$ & $-7(1)$ & $-8(1)$ \\
\hline$C(12)$ & $26(1)$ & $43(2)$ & $29(1)$ & $-10(1)$ & $-5(1)$ & $-17(1)$ \\
\hline$C(13)$ & $32(1)$ & $29(1)$ & $30(1)$ & $-7(1)$ & $-3(1)$ & $-16(1)$ \\
\hline$C(14)$ & $24(1)$ & $24(1)$ & $27(1)$ & $-2(1)$ & $-4(1)$ & $-11(1)$ \\
\hline$C(15)$ & $17(1)$ & $24(1)$ & $20(1)$ & $-2(1)$ & $-1(1)$ & $-7(1)$ \\
\hline$C(16)$ & $21(1)$ & $29(1)$ & $20(1)$ & $-3(1)$ & $-2(1)$ & $-7(1)$ \\
\hline$C(17)$ & $22(1)$ & $30(1)$ & $23(1)$ & $-6(1)$ & $0(1)$ & $-4(1)$ \\
\hline$C(18)$ & $20(1)$ & $30(1)$ & $26(1)$ & $0(1)$ & $-1(1)$ & $-3(1)$ \\
\hline$C(19)$ & $21(1)$ & $32(1)$ & $24(1)$ & $-3(1)$ & $-5(1)$ & $-4(1)$ \\
\hline$C(20)$ & $21(1)$ & $31(1)$ & $23(1)$ & $-4(1)$ & $-4(1)$ & $-7(1)$ \\
\hline$C(21)$ & $16(1)$ & $22(1)$ & $20(1)$ & $-3(1)$ & $-3(1)$ & $-7(1)$ \\
\hline$C(22)$ & $18(1)$ & $22(1)$ & $20(1)$ & $-3(1)$ & $-1(1)$ & $-8(1)$ \\
\hline$C(23)$ & $16(1)$ & $27(1)$ & $30(1)$ & $-5(1)$ & $0(1)$ & $-8(1)$ \\
\hline$C(24)$ & 18(1) & $26(1)$ & $38(1)$ & 1(1) & $-8(1)$ & $-6(1)$ \\
\hline$C(25)$ & $21(1)$ & $25(1)$ & $36(1)$ & $5(1)$ & $-7(1)$ & $-7(1)$ \\
\hline$C(26)$ & $16(1)$ & $27(1)$ & $25(1)$ & $2(1)$ & $-4(1)$ & $-7(1)$ \\
\hline$C(27)$ & $16(1)$ & $27(1)$ & $18(1)$ & $1(1)$ & $-4(1)$ & $-10(1)$ \\
\hline$C(28)$ & $18(1)$ & $28(1)$ & $24(1)$ & $-4(1)$ & $-2(1)$ & $-11(1)$ \\
\hline$C(29)$ & $27(1)$ & $31(1)$ & $26(1)$ & $-4(1)$ & $-3(1)$ & $-17(1)$ \\
\hline$C(30)$ & $22(1)$ & $44(2)$ & $28(1)$ & $-3(1)$ & $-4(1)$ & $-19(1)$ \\
\hline$C(31)$ & $17(1)$ & $36(2)$ & $26(1)$ & $-4(1)$ & $-2(1)$ & $-12(1)$ \\
\hline$C(32)$ & $18(1)$ & $27(1)$ & $22(1)$ & $-2(1)$ & $-2(1)$ & $-9(1)$ \\
\hline$C(33)$ & $26(1)$ & $28(1)$ & 19(1) & $-1(1)$ & $-5(1)$ & $-10(1)$ \\
\hline$C(34)$ & $42(1)$ & $30(2)$ & $26(1)$ & $-1(1)$ & $-14(1)$ & $-10(1)$ \\
\hline$C(35)$ & $25(1)$ & $33(1)$ & $20(1)$ & $-4(1)$ & $1(1)$ & $-13(1)$ \\
\hline$C(36)$ & $32(1)$ & $38(2)$ & $22(1)$ & $-5(1)$ & 1(1) & $-17(1)$ \\
\hline$C(37)$ & $34(1)$ & $41(2)$ & $23(1)$ & $-4(1)$ & $3(1)$ & $-16(1)$ \\
\hline$C(38)$ & $36(1)$ & $49(2)$ & $26(1)$ & $-11(1)$ & $5(1)$ & $-18(1)$ \\
\hline
\end{tabular}




\begin{tabular}{|c|c|c|c|c|c|c|}
\hline$C(39)$ & $38(1)$ & $35(2)$ & $31(1)$ & $-12(1)$ & $4(1)$ & $-16(1)$ \\
\hline$C(40)$ & $28(1)$ & $33(2)$ & $27(1)$ & $-6(1)$ & $0(1)$ & $-13(1)$ \\
\hline$C(41)$ & $22(1)$ & $30(1)$ & $19(1)$ & $-1(1)$ & $-3(1)$ & $-12(1)$ \\
\hline$C(42)$ & $32(1)$ & $44(2)$ & $23(1)$ & 1(1) & $-7(1)$ & $-21(1)$ \\
\hline$C(43)$ & $33(1)$ & $47(2)$ & $29(1)$ & $-2(1)$ & $-7(1)$ & $-23(1)$ \\
\hline$C(44)$ & $23(1)$ & $39(2)$ & $31(1)$ & $2(1)$ & $-3(1)$ & $-17(1)$ \\
\hline$C(45)$ & $21(1)$ & $32(1)$ & $24(1)$ & $-1(1)$ & $-1(1)$ & $-10(1)$ \\
\hline$C(46)$ & $24(1)$ & $32(1)$ & $21(1)$ & $-3(1)$ & $-1(1)$ & $-12(1)$ \\
\hline$C(47)$ & $20(1)$ & $30(1)$ & $24(1)$ & $-4(1)$ & $-4(1)$ & $-10(1)$ \\
\hline$C(48)$ & $26(1)$ & $29(1)$ & $28(1)$ & $-2(1)$ & $-8(1)$ & $-11(1)$ \\
\hline$C(49)$ & $29(1)$ & $30(1)$ & $32(1)$ & $-5(1)$ & $-11(1)$ & $-9(1)$ \\
\hline$C(50)$ & $24(1)$ & $42(2)$ & $38(1)$ & $-13(1)$ & $-10(1)$ & $-7(1)$ \\
\hline$C(51)$ & $22(1)$ & $47(2)$ & $34(1)$ & $-10(1)$ & $-5(1)$ & $-16(1)$ \\
\hline$C(52)$ & $24(1)$ & $36(2)$ & $27(1)$ & $-4(1)$ & $-2(1)$ & $-14(1)$ \\
\hline$C(53)$ & $34(1)$ & $28(1)$ & $20(1)$ & $0(1)$ & $-4(1)$ & $-16(1)$ \\
\hline$C(54)$ & $36(1)$ & $38(2)$ & $31(1)$ & $-5(1)$ & $3(1)$ & $-20(1)$ \\
\hline$C(55)$ & $43(2)$ & $47(2)$ & $38(2)$ & $-10(1)$ & $8(1)$ & $-27(1)$ \\
\hline$C(56)$ & $72(2)$ & $53(2)$ & $25(1)$ & $1(1)$ & $2(1)$ & $-42(2)$ \\
\hline$C(57)$ & $61(2)$ & $45(2)$ & $33(1)$ & $12(1)$ & $-15(1)$ & $-31(2)$ \\
\hline$C(58)$ & $47(2)$ & $40(2)$ & $27(1)$ & $9(1)$ & $-11(1)$ & $-22(1)$ \\
\hline$C(59)$ & $23(1)$ & $27(1)$ & 19(1) & $-2(1)$ & $-5(1)$ & $-5(1)$ \\
\hline$C(60)$ & $24(1)$ & $35(2)$ & $27(1)$ & $-3(1)$ & $-4(1)$ & $-11(1)$ \\
\hline$C(61)$ & $26(1)$ & $44(2)$ & $37(1)$ & $-7(1)$ & $-2(1)$ & $-11(1)$ \\
\hline$C(62)$ & $28(1)$ & $48(2)$ & $38(2)$ & $-12(1)$ & $3(1)$ & $-6(1)$ \\
\hline$C(63)$ & $34(1)$ & $34(2)$ & $34(1)$ & $-8(1)$ & $-7(1)$ & $-4(1)$ \\
\hline$C(64)$ & $31(1)$ & $36(2)$ & $25(1)$ & $-5(1)$ & $-6(1)$ & $-11(1)$ \\
\hline
\end{tabular}




\subsubsection{Crystal Structure Determination of $\mathbf{2}_{2}$}

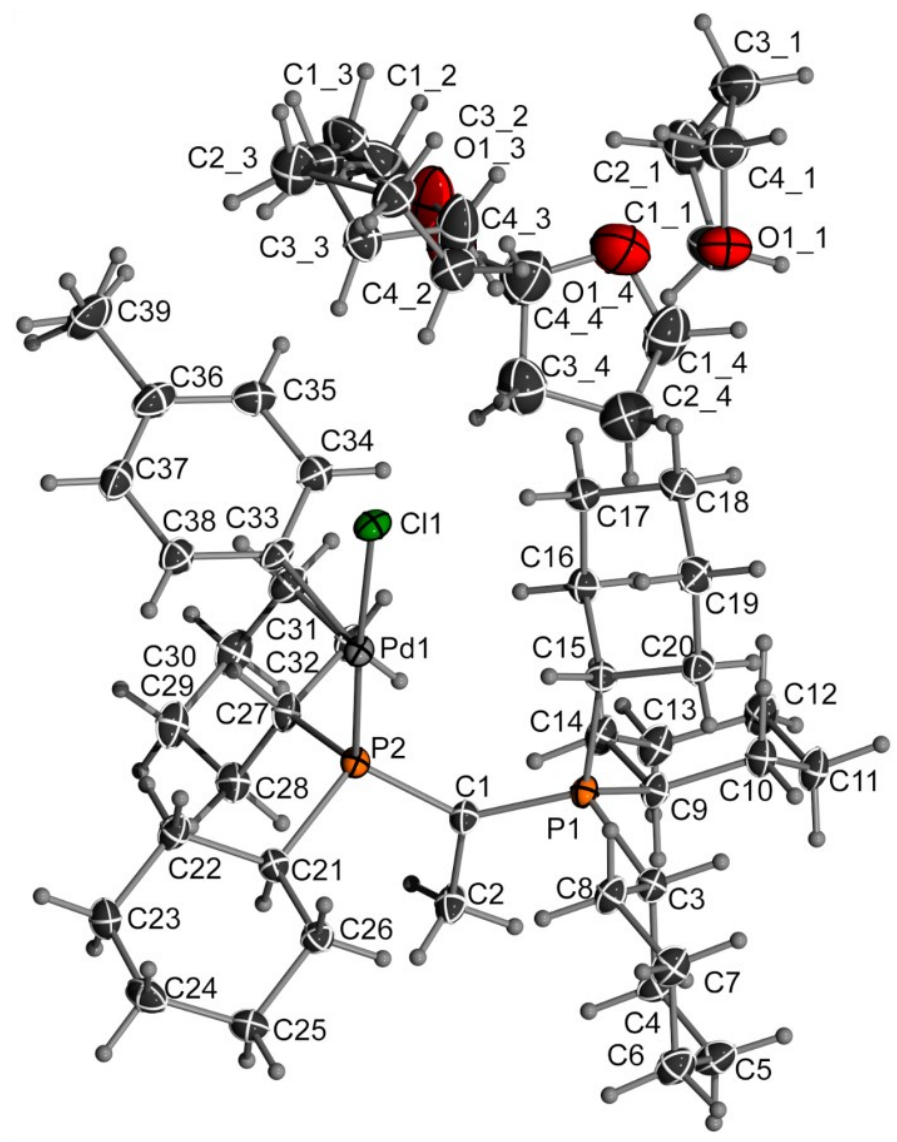

Figure S9. ORTEP Plot of $\mathbf{2 2}^{\text {Tol }}$. Ellipsoids are drawn at the $50 \%$ probability level.

Table S6. Atomic coordinates $\left(\times 10^{4}\right)$ and equivalent isotropic displacement parameters $\left(\AA^{2} \times 10^{3}\right)$ for $\mathbf{2 2}^{\text {Tol }}$. U(eq) is defined as one third of the trace of the orthogonalized $\mathrm{U}^{\mathrm{ij}}$ tensor.

\begin{tabular}{|c|c|c|c|c|}
\hline & $x$ & $y$ & z & $\mathrm{U}(\mathrm{eq})$ \\
\hline $\mathrm{Pd}(1)$ & $4166(1)$ & $3630(1)$ & 4491(1) & 15(1) \\
\hline $\mathrm{Cl}(1)$ & $4273(1)$ & $4868(1)$ & $3950(1)$ & $20(1)$ \\
\hline$P(1)$ & $6970(1)$ & $2708(1)$ & $5129(1)$ & $16(1)$ \\
\hline$P(2)$ & $4130(1)$ & $2382(1)$ & 4891(1) & $15(1)$ \\
\hline$C(1)$ & $5522(2)$ & $2219(1)$ & $5220(1)$ & $18(1)$ \\
\hline$C(2)$ & $5435(2)$ & $1509(2)$ & $5663(2)$ & $24(1)$ \\
\hline$C(3)$ & $8274(2)$ & $3653(1)$ & $6351(1)$ & 19(1) \\
\hline$C(4)$ & $8498(2)$ & $3210(2)$ & $7033(2)$ & $22(1)$ \\
\hline$C(5)$ & $9609(2)$ & $4011(2)$ & $8002(2)$ & $28(1)$ \\
\hline$C(6)$ & $9430(2)$ & $5026(2)$ & $8585(2)$ & $28(1)$ \\
\hline$C(7)$ & $9200(2)$ & $5460(2)$ & $7904(2)$ & $26(1)$ \\
\hline$C(8)$ & $8080(2)$ & $4660(1)$ & $6944(2)$ & $22(1)$ \\
\hline$C(9)$ & $7507(2)$ & $1631(1)$ & $4556(1)$ & 19(1) \\
\hline$C(10)$ & $8723(2)$ & 1916(2) & $4315(2)$ & $23(1)$ \\
\hline$C(11)$ & $9134(2)$ & $1001(2)$ & $3951(2)$ & $25(1)$ \\
\hline$C(12)$ & $8109(2)$ & $15(2)$ & $3036(2)$ & $27(1)$ \\
\hline$C(13)$ & $6901(2)$ & $-268(2)$ & $3268(2)$ & $25(1)$ \\
\hline$C(14)$ & $6470(2)$ & $632(1)$ & $3639(2)$ & $22(1)$ \\
\hline$C(15)$ & $7003(2)$ & $3389(1)$ & $4424(1)$ & $19(1)$ \\
\hline$C(16)$ & $6341(2)$ & $2643(2)$ & $3277(1)$ & $22(1)$ \\
\hline
\end{tabular}




\begin{tabular}{|c|c|c|c|c|}
\hline$C(17)$ & $6225(2)$ & $3258(2)$ & $2759(2)$ & $24(1)$ \\
\hline$C(18)$ & $7503(2)$ & $4050(2)$ & 2971(2) & $28(1)$ \\
\hline$C(19)$ & $8153(2)$ & $4778(2)$ & $4107(2)$ & $27(1)$ \\
\hline$C(20)$ & $8305(2)$ & $4168(2)$ & $4629(2)$ & $22(1)$ \\
\hline$C(21)$ & $3556(2)$ & $2630(1)$ & $5983(1)$ & $17(1)$ \\
\hline$C(22)$ & $2321(2)$ & $2770(2)$ & $5826(2)$ & $22(1)$ \\
\hline$C(23)$ & $1779(2)$ & $2782(2)$ & $6654(2)$ & $27(1)$ \\
\hline$C(24)$ & $2705(2)$ & $3631(2)$ & $7703(2)$ & $28(1)$ \\
\hline$C(25)$ & $3969(2)$ & $3539(2)$ & $7848(2)$ & $25(1)$ \\
\hline$C(26)$ & $4497(2)$ & $3532(2)$ & $7010(1)$ & $21(1)$ \\
\hline$C(27)$ & $2844(2)$ & $1080(1)$ & $3887(1)$ & $19(1)$ \\
\hline$C(28)$ & $2228(2)$ & $280(1)$ & $4175(2)$ & $22(1)$ \\
\hline$C(29)$ & $1111(2)$ & $-646(2)$ & $3275(2)$ & $26(1)$ \\
\hline$C(30)$ & 1483(2) & $-1168(2)$ & $2343(2)$ & $27(1)$ \\
\hline$C(31)$ & $2122(2)$ & $-370(2)$ & $2073(2)$ & $27(1)$ \\
\hline$C(32)$ & $3243(2)$ & $547(1)$ & $2976(1)$ & $21(1)$ \\
\hline$C(33)$ & $2684(2)$ & $2672(1)$ & $3245(1)$ & $20(1)$ \\
\hline$C(34)$ & $2761(2)$ & 2192(2) & $2280(2)$ & $24(1)$ \\
\hline$C(35)$ & $1709(2)$ & $1706(2)$ & $1430(2)$ & $28(1)$ \\
\hline$C(36)$ & $552(2)$ & 1694(2) & $1517(2)$ & $31(1)$ \\
\hline$C(37)$ & $471(2)$ & $2178(2)$ & $2478(2)$ & $29(1)$ \\
\hline$C(38)$ & $1516(2)$ & $2660(2)$ & $3335(2)$ & $24(1)$ \\
\hline$C(39)$ & $-580(2)$ & $1195(2)$ & $592(2)$ & $45(1)$ \\
\hline 011 & $7287(2)$ & $3823(1)$ & $727(1)$ & $44(1)$ \\
\hline C11 & $6924(3)$ & $2721(2)$ & $224(2)$ & $51(1)$ \\
\hline $\mathrm{C} 21$ & $6149(3)$ & $2280(2)$ & $-854(2)$ & $50(1)$ \\
\hline C31 & $6771(3)$ & $3153(2)$ & $-1022(2)$ & $46(1)$ \\
\hline C41 & 7064(3) & $4108(2)$ & $-13(2)$ & $43(1)$ \\
\hline O12 & $3753(5)$ & 2464(3) & $275(4)$ & $47(1)$ \\
\hline C12 & $2652(8)$ & $2149(5)$ & $-527(4)$ & $50(2)$ \\
\hline $\mathrm{C} 22$ & $2103(7)$ & $2955(7)$ & $-104(6)$ & $43(2)$ \\
\hline $\mathrm{C} 32$ & $3313(4)$ & $3929(3)$ & $576(4)$ & $36(1)$ \\
\hline $\mathrm{C} 42$ & $4126(5)$ & $3529(4)$ & $998(4)$ & $36(1)$ \\
\hline O13 & $3339(10)$ & $2316(7)$ & $-165(9)$ & $61(3)$ \\
\hline C13 & $2152(12)$ & 2191(10) & $-695(9)$ & $43(2)$ \\
\hline $\mathrm{C} 23$ & $1856(11)$ & $3070(12)$ & $17(11)$ & $39(3)$ \\
\hline C33 & $3048(7)$ & $3808(5)$ & $934(6)$ & $31(2)$ \\
\hline $\mathrm{C} 43$ & 4015(9) & $3419(9)$ & $563(10)$ & $58(3)$ \\
\hline O14 & $5068(5)$ & $-121(5)$ & $-1029(4)$ & $76(1)$ \\
\hline C14 & $5801(13)$ & $-362(13)$ & $-421(9)$ & $52(3)$ \\
\hline $\mathrm{C} 24$ & $5735(11)$ & $244(11)$ & $619(8)$ & $56(3)$ \\
\hline C34 & $4412(13)$ & $208(15)$ & $412(10)$ & $52(3)$ \\
\hline C44 & $4081(12)$ & $-7(12)$ & $-652(9)$ & $60(3)$ \\
\hline
\end{tabular}

Table S7. Anisotropic displacement parameters $\left(\AA^{2} \times 10^{3}\right)$ for $\mathbf{2}^{\mathrm{Tol}}$. The anisotropic displacement factor exponent takes the form: $-2 \pi^{2}\left[h^{2} a \cdot{ }^{2} U^{11}+\ldots+2 h k a \cdot b \cdot U^{12}\right]$.

\begin{tabular}{ccccccc} 
& $\mathrm{U}^{11}$ & $\mathrm{U}^{22}$ & $\mathrm{U}^{33}$ & $\mathrm{U}^{23}$ & $\mathrm{U}^{13}$ & $\mathrm{U}^{12}$ \\
\hline \hline $\mathrm{Pd}(1)$ & $14(1)$ & $13(1)$ & $18(1)$ & $8(1)$ & $4(1)$ & $5(1)$ \\
$\mathrm{Cl}(1)$ & $21(1)$ & $15(1)$ & $22(1)$ & $10(1)$ & $3(1)$ & $6(1)$
\end{tabular}




\begin{tabular}{|c|c|c|c|c|c|c|}
\hline$P(1)$ & $15(1)$ & $15(1)$ & 19(1) & $9(1)$ & $5(1)$ & $6(1)$ \\
\hline$P(2)$ & $14(1)$ & $13(1)$ & $17(1)$ & $8(1)$ & $5(1)$ & $5(1)$ \\
\hline$C(1)$ & $15(1)$ & $18(1)$ & $23(1)$ & 11(1) & $6(1)$ & $6(1)$ \\
\hline$C(2)$ & $20(1)$ & $25(1)$ & $36(1)$ & $21(1)$ & $10(1)$ & $11(1)$ \\
\hline$C(3)$ & $17(1)$ & 19(1) & 21(1) & $10(1)$ & $5(1)$ & $7(1)$ \\
\hline$C(4)$ & $25(1)$ & 21(1) & $23(1)$ & $12(1)$ & $5(1)$ & $10(1)$ \\
\hline$C(5)$ & $31(1)$ & $28(1)$ & $24(1)$ & $12(1)$ & $4(1)$ & $15(1)$ \\
\hline$C(6)$ & $28(1)$ & $26(1)$ & 21(1) & $7(1)$ & $3(1)$ & $11(1)$ \\
\hline$C(7)$ & $25(1)$ & 18(1) & $25(1)$ & $7(1)$ & $4(1)$ & $8(1)$ \\
\hline$C(8)$ & $21(1)$ & $20(1)$ & $24(1)$ & $9(1)$ & $5(1)$ & $9(1)$ \\
\hline$C(9)$ & $17(1)$ & $17(1)$ & $21(1)$ & $9(1)$ & $6(1)$ & $8(1)$ \\
\hline$C(10)$ & $19(1)$ & 21(1) & $26(1)$ & 11(1) & $8(1)$ & $9(1)$ \\
\hline$C(11)$ & $18(1)$ & $24(1)$ & $29(1)$ & $10(1)$ & $7(1)$ & $11(1)$ \\
\hline$C(12)$ & $25(1)$ & $24(1)$ & $25(1)$ & $6(1)$ & $7(1)$ & $14(1)$ \\
\hline$C(13)$ & 21(1) & 18(1) & $27(1)$ & $7(1)$ & $3(1)$ & $7(1)$ \\
\hline$C(14)$ & $18(1)$ & 19(1) & $25(1)$ & $9(1)$ & $4(1)$ & $7(1)$ \\
\hline$C(15)$ & $18(1)$ & $19(1)$ & $22(1)$ & $12(1)$ & $7(1)$ & $8(1)$ \\
\hline$C(16)$ & $20(1)$ & $22(1)$ & $22(1)$ & 11(1) & $6(1)$ & $7(1)$ \\
\hline$C(17)$ & $23(1)$ & $29(1)$ & $23(1)$ & $16(1)$ & $7(1)$ & $10(1)$ \\
\hline$C(18)$ & $27(1)$ & $34(1)$ & $32(1)$ & $23(1)$ & $11(1)$ & $10(1)$ \\
\hline$C(19)$ & $23(1)$ & $25(1)$ & $34(1)$ & $20(1)$ & $7(1)$ & $5(1)$ \\
\hline$C(20)$ & $18(1)$ & $23(1)$ & $27(1)$ & $15(1)$ & $6(1)$ & $7(1)$ \\
\hline$C(21)$ & $18(1)$ & $16(1)$ & $20(1)$ & $10(1)$ & $8(1)$ & $8(1)$ \\
\hline$C(22)$ & $20(1)$ & $23(1)$ & $24(1)$ & 11(1) & $6(1)$ & 11(1) \\
\hline$C(23)$ & $23(1)$ & $33(1)$ & $31(1)$ & $17(1)$ & 13(1) & $14(1)$ \\
\hline$C(24)$ & $37(1)$ & $30(1)$ & $26(1)$ & $16(1)$ & $18(1)$ & $19(1)$ \\
\hline$C(25)$ & $30(1)$ & $25(1)$ & $20(1)$ & $12(1)$ & $9(1)$ & 11(1) \\
\hline$C(26)$ & $21(1)$ & $22(1)$ & $19(1)$ & $10(1)$ & $5(1)$ & $6(1)$ \\
\hline$C(27)$ & $15(1)$ & 14(1) & $25(1)$ & $8(1)$ & $5(1)$ & $5(1)$ \\
\hline$C(28)$ & $21(1)$ & $17(1)$ & $28(1)$ & $12(1)$ & $8(1)$ & $6(1)$ \\
\hline$C(29)$ & $19(1)$ & $17(1)$ & $37(1)$ & $12(1)$ & $6(1)$ & $5(1)$ \\
\hline$C(30)$ & $22(1)$ & $16(1)$ & $30(1)$ & $7(1)$ & $2(1)$ & $3(1)$ \\
\hline$C(31)$ & $26(1)$ & $22(1)$ & $23(1)$ & $7(1)$ & $2(1)$ & $6(1)$ \\
\hline$C(32)$ & $18(1)$ & $18(1)$ & $22(1)$ & $8(1)$ & $4(1)$ & $6(1)$ \\
\hline$C(33)$ & $17(1)$ & $17(1)$ & $23(1)$ & $13(1)$ & $0(1)$ & 4(1) \\
\hline$C(34)$ & $23(1)$ & $20(1)$ & $25(1)$ & $12(1)$ & $4(1)$ & $4(1)$ \\
\hline$C(35)$ & $33(1)$ & $25(1)$ & $22(1)$ & $12(1)$ & $5(1)$ & $8(1)$ \\
\hline$C(36)$ & $26(1)$ & $26(1)$ & $29(1)$ & $15(1)$ & $-3(1)$ & 2(1) \\
\hline$C(37)$ & $19(1)$ & $31(1)$ & $36(1)$ & $18(1)$ & $4(1)$ & $7(1)$ \\
\hline$C(38)$ & $22(1)$ & $22(1)$ & $26(1)$ & $12(1)$ & $5(1)$ & $6(1)$ \\
\hline$C(39)$ & $31(1)$ & $50(2)$ & $35(1)$ & $17(1)$ & $-6(1)$ & $7(1)$ \\
\hline 011 & $52(1)$ & $44(1)$ & $33(1)$ & $21(1)$ & 11(1) & $17(1)$ \\
\hline C11 & $68(2)$ & $47(2)$ & $44(2)$ & $28(1)$ & $23(1)$ & $24(1)$ \\
\hline C21 & $43(2)$ & $49(2)$ & $44(2)$ & $13(1)$ & $18(1)$ & $18(1)$ \\
\hline C31 & $53(2)$ & $60(2)$ & $34(1)$ & $24(1)$ & $15(1)$ & $33(1)$ \\
\hline C41 & $50(2)$ & $47(1)$ & $35(1)$ & $22(1)$ & $14(1)$ & $23(1)$ \\
\hline 012 & $52(3)$ & $33(2)$ & $55(3)$ & $16(2)$ & $23(2)$ & $22(2)$ \\
\hline C12 & $52(5)$ & $38(2)$ & $31(3)$ & $6(2)$ & $18(3)$ & $-2(3)$ \\
\hline $\mathrm{C} 22$ & $39(3)$ & $46(3)$ & $36(3)$ & $25(2)$ & $4(2)$ & $6(2)$ \\
\hline C32 & $33(2)$ & $33(2)$ & $38(2)$ & $18(2)$ & $7(2)$ & $11(2)$ \\
\hline
\end{tabular}




\begin{tabular}{ccccccc} 
C42 & $39(2)$ & $33(2)$ & $35(3)$ & $17(2)$ & $10(2)$ & $16(2)$ \\
O13 & $49(5)$ & $48(4)$ & $67(6)$ & $12(4)$ & $19(4)$ & $26(4)$ \\
C13 & $47(6)$ & $40(4)$ & $34(4)$ & $13(3)$ & $19(4)$ & $14(4)$ \\
C23 & $40(5)$ & $30(4)$ & $33(4)$ & $5(3)$ & $13(4)$ & $15(3)$ \\
C33 & $30(3)$ & $29(3)$ & $34(4)$ & $16(3)$ & $12(3)$ & $11(3)$ \\
C43 & $39(4)$ & $59(5)$ & $55(6)$ & $10(5)$ & $17(5)$ & $23(3)$ \\
O14 & $78(4)$ & $105(4)$ & $52(3)$ & $39(3)$ & $28(3)$ & $46(3)$ \\
C14 & $37(4)$ & $43(6)$ & $48(4)$ & $8(3)$ & $3(3)$ & $10(4)$ \\
C24 & $54(5)$ & $63(6)$ & $50(4)$ & $31(4)$ & $13(3)$ & $22(4)$ \\
C34 & $49(6)$ & $47(5)$ & $53(5)$ & $20(4)$ & $19(4)$ & $18(4)$ \\
C44 & $53(5)$ & $67(7)$ & $61(4)$ & $33(4)$ & $17(4)$ & $29(4)$ \\
\hline
\end{tabular}

\subsubsection{Crystal Structure Determination of $3^{\text {Et }}$}

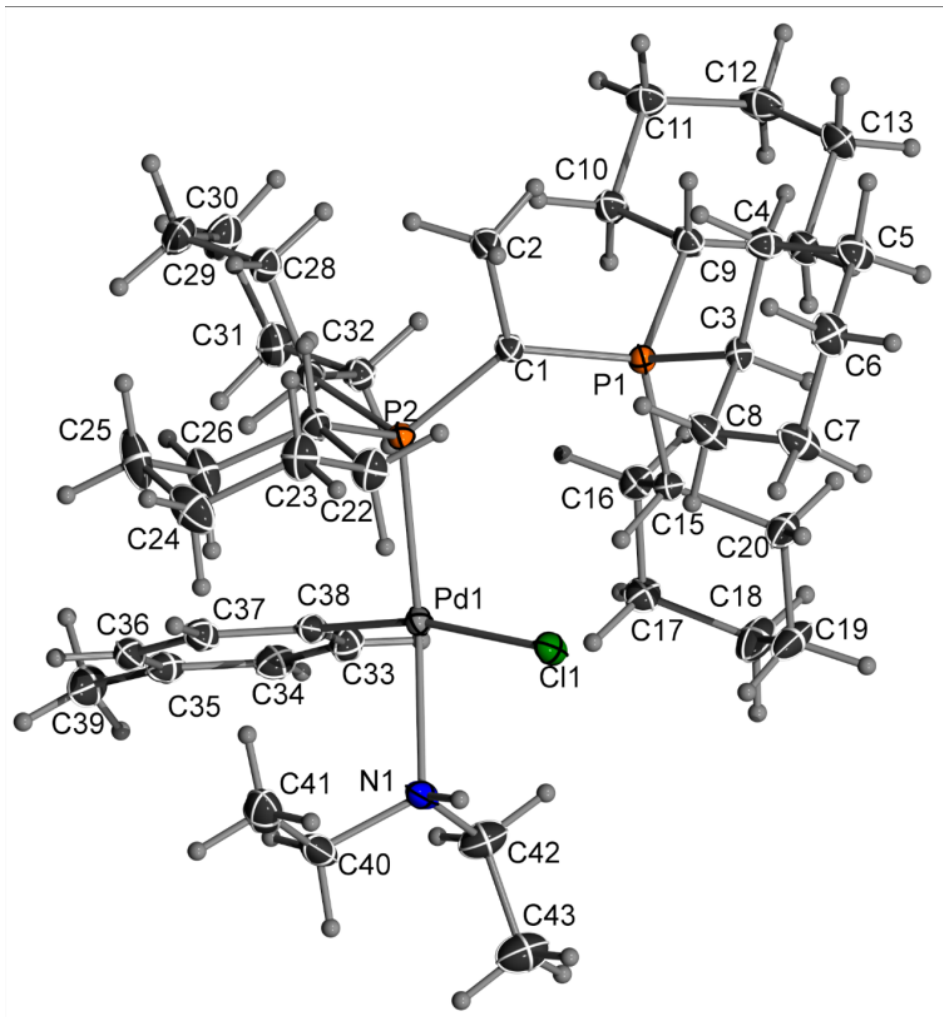

Figure S10. ORTEP Plot of $3^{\mathrm{Et}}$. Ellipsoids are drawn at the $50 \%$ probability level.

Table S8. Atomic coordinates $\left(\times 10^{4}\right)$ and equivalent isotropic displacement parameters $\left(\AA^{2} \times 10^{3}\right)$ for $3^{\text {Et }}$. $U($ eq $)$ is defined as one third of the trace of the orthogonalized $U^{i j}$ tensor.

\begin{tabular}{ccccc} 
& $\mathrm{x}$ & $\mathrm{y}$ & $\mathrm{z}$ & $\mathrm{U}(\mathrm{eq})$ \\
\hline \hline $\mathrm{Pd}(1)$ & $6582(1)$ & $5476(1)$ & $1660(1)$ & $13(1)$ \\
$\mathrm{Cl}(1)$ & $4598(1)$ & $6100(1)$ & $916(1)$ & $18(1)$ \\
$\mathrm{P}(1)$ & $3609(1)$ & $7991(1)$ & $3060(1)$ & $12(1)$ \\
$\mathrm{P}(2)$ & $6355(1)$ & $7210(1)$ & $2175(1)$ & $12(1)$ \\
$\mathrm{N}(1)$ & $6852(2)$ & $3947(2)$ & $1011(1)$ & $18(1)$ \\
$\mathrm{C}(1)$ & $4908(2)$ & $8192(2)$ & $2588(2)$ & $14(1)$
\end{tabular}




\begin{tabular}{|c|c|c|c|c|}
\hline$C(2)$ & $4727(2)$ & $9438(2)$ & 2522(2) & $18(1)$ \\
\hline$C(3)$ & $2056(2)$ & $8739(2)$ & 2532(2) & $15(1)$ \\
\hline$C(4)$ & 1382(2) & $10000(2)$ & 2681(2) & $19(1)$ \\
\hline$C(5)$ & $58(2)$ & $10441(2)$ & 2293(2) & $22(1)$ \\
\hline$C(6)$ & $114(2)$ & $10209(2)$ & 1336(2) & $24(1)$ \\
\hline$C(7)$ & $834(2)$ & $8975(2)$ & $1177(2)$ & $26(1)$ \\
\hline$C(8)$ & $2161(2)$ & $8544(2)$ & 1563(2) & $23(1)$ \\
\hline$C(9)$ & $3237(2)$ & $8598(2)$ & $4148(2)$ & $16(1)$ \\
\hline$C(10)$ & $4396(2)$ & $8354(2)$ & $4708(2)$ & 19(1) \\
\hline$C(11)$ & $4023(2)$ & $9042(2)$ & $5519(2)$ & $23(1)$ \\
\hline$C(12)$ & $2937(2)$ & $8838(2)$ & 6034(2) & $25(1)$ \\
\hline$C(13)$ & $1790(2)$ & $9067(2)$ & $5471(2)$ & $24(1)$ \\
\hline$C(14)$ & 2153(2) & $8378(2)$ & $4660(2)$ & 19(1) \\
\hline$C(15)$ & $3824(2)$ & $6508(2)$ & $3105(2)$ & 14(1) \\
\hline$C(16)$ & $4671(2)$ & $5846(2)$ & $3841(2)$ & $17(1)$ \\
\hline$C(17)$ & $5000(2)$ & $4595(2)$ & $3749(2)$ & $19(1)$ \\
\hline$C(18)$ & $3805(2)$ & $4320(2)$ & $3774(2)$ & $28(1)$ \\
\hline$C(19)$ & $2920(2)$ & 4993(2) & $3059(2)$ & $28(1)$ \\
\hline$C(20)$ & 2601(2) & $6251(2)$ & $3109(2)$ & $21(1)$ \\
\hline$C(21)$ & $6764(2)$ & $7959(2)$ & $1213(2)$ & $16(1)$ \\
\hline$C(22)$ & $5824(2)$ & 8152(2) & $488(2)$ & $22(1)$ \\
\hline$C(23)$ & $6148(2)$ & $8789(2)$ & $-291(2)$ & $24(1)$ \\
\hline$C(24)$ & 7495(3) & $8174(2)$ & $-627(2)$ & $32(1)$ \\
\hline$C(25)$ & $8427(2)$ & $8022(2)$ & $90(2)$ & $33(1)$ \\
\hline$C(26)$ & $8130(2)$ & $7378(2)$ & $868(2)$ & $25(1)$ \\
\hline$C(27)$ & $7679(2)$ & 7096(2) & 2895(2) & $15(1)$ \\
\hline$C(28)$ & $7876(2)$ & $8189(2)$ & $3048(2)$ & 19(1) \\
\hline$C(29)$ & $9117(2)$ & $7931(2)$ & $3518(2)$ & $23(1)$ \\
\hline$C(30)$ & $9120(2)$ & $7298(2)$ & $4379(2)$ & $27(1)$ \\
\hline$C(31)$ & $8838(2)$ & $6243(2)$ & $4260(2)$ & $22(1)$ \\
\hline$C(32)$ & $7611(2)$ & $6509(2)$ & $3771(2)$ & $17(1)$ \\
\hline$C(33)$ & $8271(2)$ & $4658(2)$ & 2208(2) & $17(1)$ \\
\hline$C(34)$ & $8364(2)$ & $3952(2)$ & 2941(2) & $20(1)$ \\
\hline$C(35)$ & $9535(2)$ & $3268(2)$ & $3273(2)$ & $22(1)$ \\
\hline$C(36)$ & $10676(2)$ & $3257(2)$ & $2884(2)$ & $21(1)$ \\
\hline$C(37)$ & $10598(2)$ & $3944(2)$ & $2156(2)$ & $20(1)$ \\
\hline$C(38)$ & $9426(2)$ & $4630(2)$ & 1825(2) & $18(1)$ \\
\hline$C(39)$ & $11945(2)$ & 2532(2) & $3253(2)$ & $27(1)$ \\
\hline$C(40)$ & $8013(2)$ & $3500(2)$ & $451(2)$ & $26(1)$ \\
\hline$C(41)$ & $8124(3)$ & $4378(2)$ & $-199(2)$ & $32(1)$ \\
\hline$C(42)$ & $6639(3)$ & $3080(2)$ & 1590(2) & $28(1)$ \\
\hline$C(43)$ & 6349(3) & $2188(2)$ & $1156(2)$ & $35(1)$ \\
\hline
\end{tabular}

Table S9. Anisotropic displacement parameters $\left(\AA^{2} \times 10^{3}\right)$ for $3^{\mathrm{Et}}$. The anisotropic displacement factor exponent takes the form: $-2 \pi^{2}\left[h^{2} a \cdot{ }^{2} U^{11}+\ldots+2 h k a \cdot b \cdot U^{12}\right]$.

\begin{tabular}{ccccccc} 
& $\mathrm{U}^{11}$ & $\mathrm{U}^{22}$ & $\mathrm{U}^{33}$ & $\mathrm{U}^{23}$ & $\mathrm{U}^{13}$ & $\mathrm{U}^{12}$ \\
\hline $\mathrm{Pd}(1)$ & $11(1)$ & $13(1)$ & $14(1)$ & $-2(1)$ & $-1(1)$ & $-3(1)$ \\
$\mathrm{Cl}(1)$ & $15(1)$ & $18(1)$ & $18(1)$ & $-4(1)$ & $-3(1)$ & $-3(1)$ \\
$\mathrm{P}(1)$ & $11(1)$ & $13(1)$ & $12(1)$ & $-1(1)$ & $1(1)$ & $-4(1)$
\end{tabular}




\begin{tabular}{|c|c|c|c|c|c|c|}
\hline$P(2)$ & 11(1) & $13(1)$ & $13(1)$ & $-1(1)$ & $0(1)$ & $-4(1)$ \\
\hline$N(1)$ & $15(1)$ & $17(1)$ & 19(1) & $-7(1)$ & $-1(1)$ & $-2(1)$ \\
\hline$C(1)$ & $12(1)$ & $12(1)$ & $17(1)$ & $-1(1)$ & 1(1) & $-3(1)$ \\
\hline$C(2)$ & $15(1)$ & $14(1)$ & $24(1)$ & $-2(1)$ & $3(1)$ & $-5(1)$ \\
\hline$C(3)$ & $12(1)$ & $16(1)$ & $17(1)$ & $0(1)$ & $0(1)$ & $-4(1)$ \\
\hline$C(4)$ & $19(1)$ & $16(1)$ & 21(1) & $-2(1)$ & $-4(1)$ & $-4(1)$ \\
\hline$C(5)$ & $17(1)$ & $19(1)$ & $26(1)$ & $-1(1)$ & $-2(1)$ & $-1(1)$ \\
\hline$C(6)$ & $19(1)$ & $25(1)$ & $24(1)$ & $2(1)$ & $-4(1)$ & $-4(1)$ \\
\hline$C(7)$ & $20(1)$ & $28(1)$ & $23(1)$ & $-7(1)$ & $-6(1)$ & 1(1) \\
\hline$C(8)$ & $18(1)$ & $26(1)$ & $17(1)$ & $-6(1)$ & $-2(1)$ & $2(1)$ \\
\hline $\mathrm{C}(9)$ & $16(1)$ & $17(1)$ & $15(1)$ & $-2(1)$ & 2(1) & $-6(1)$ \\
\hline$C(10)$ & $17(1)$ & $20(1)$ & $18(1)$ & $-4(1)$ & $-1(1)$ & $-6(1)$ \\
\hline$C(11)$ & $24(1)$ & $25(1)$ & $19(1)$ & $-7(1)$ & $0(1)$ & $-9(1)$ \\
\hline$C(12)$ & $32(1)$ & $25(1)$ & $17(1)$ & $-6(1)$ & $4(1)$ & $-10(1)$ \\
\hline$C(13)$ & $24(1)$ & $27(1)$ & 21(1) & $-7(1)$ & $9(1)$ & $-10(1)$ \\
\hline$C(14)$ & $18(1)$ & $21(1)$ & $18(1)$ & $-4(1)$ & $4(1)$ & $-7(1)$ \\
\hline$C(15)$ & $14(1)$ & $14(1)$ & $16(1)$ & $-2(1)$ & $0(1)$ & $-5(1)$ \\
\hline$C(16)$ & $18(1)$ & $17(1)$ & $15(1)$ & 1(1) & $-2(1)$ & $-7(1)$ \\
\hline$C(17)$ & $20(1)$ & $17(1)$ & 19(1) & $2(1)$ & $-3(1)$ & $-6(1)$ \\
\hline$C(18)$ & $23(1)$ & $20(1)$ & $42(2)$ & $7(1)$ & $-7(1)$ & $-10(1)$ \\
\hline$C(19)$ & $24(1)$ & 19(1) & $45(2)$ & $3(1)$ & $-13(1)$ & $-12(1)$ \\
\hline$C(20)$ & $17(1)$ & $17(1)$ & $30(1)$ & $2(1)$ & $-5(1)$ & $-8(1)$ \\
\hline$C(21)$ & $15(1)$ & $17(1)$ & $17(1)$ & $0(1)$ & $2(1)$ & $-5(1)$ \\
\hline$C(22)$ & $22(1)$ & $25(1)$ & $18(1)$ & $4(1)$ & $-2(1)$ & $-10(1)$ \\
\hline$C(23)$ & $24(1)$ & $28(1)$ & $20(1)$ & $6(1)$ & $-2(1)$ & $-10(1)$ \\
\hline$C(24)$ & $35(1)$ & $30(1)$ & $22(1)$ & $4(1)$ & $8(1)$ & $-5(1)$ \\
\hline$C(25)$ & 19(1) & $40(1)$ & $30(2)$ & $13(1)$ & $8(1)$ & $-3(1)$ \\
\hline$C(26)$ & $18(1)$ & $27(1)$ & $24(1)$ & $7(1)$ & $4(1)$ & $-1(1)$ \\
\hline$C(27)$ & $12(1)$ & $16(1)$ & $16(1)$ & $-1(1)$ & $-1(1)$ & $-5(1)$ \\
\hline$C(28)$ & $17(1)$ & $19(1)$ & 21(1) & $-2(1)$ & $-2(1)$ & $-7(1)$ \\
\hline$C(29)$ & 19(1) & $29(1)$ & $27(1)$ & $-4(1)$ & $-2(1)$ & $-14(1)$ \\
\hline$C(30)$ & $26(1)$ & $40(1)$ & $22(1)$ & $-2(1)$ & $-6(1)$ & $-18(1)$ \\
\hline$C(31)$ & $18(1)$ & $28(1)$ & $19(1)$ & $2(1)$ & $-5(1)$ & $-7(1)$ \\
\hline$C(32)$ & $16(1)$ & $17(1)$ & $18(1)$ & $0(1)$ & $-1(1)$ & $-6(1)$ \\
\hline$C(33)$ & $15(1)$ & $15(1)$ & $20(1)$ & $-6(1)$ & $-2(1)$ & $-3(1)$ \\
\hline$C(34)$ & $17(1)$ & $20(1)$ & $22(1)$ & $0(1)$ & $-3(1)$ & $-7(1)$ \\
\hline$C(35)$ & $23(1)$ & $18(1)$ & $24(1)$ & 1(1) & $-6(1)$ & $-7(1)$ \\
\hline$C(36)$ & $18(1)$ & $15(1)$ & $27(1)$ & $-4(1)$ & $-8(1)$ & $-3(1)$ \\
\hline$C(37)$ & $16(1)$ & $20(1)$ & $23(1)$ & $-6(1)$ & $0(1)$ & $-5(1)$ \\
\hline$C(38)$ & $17(1)$ & $16(1)$ & $20(1)$ & $-2(1)$ & $-1(1)$ & $-4(1)$ \\
\hline$C(39)$ & $19(1)$ & $21(1)$ & $38(2)$ & $0(1)$ & $-10(1)$ & $-3(1)$ \\
\hline$C(40)$ & $20(1)$ & $27(1)$ & $28(1)$ & $-14(1)$ & $2(1)$ & $-3(1)$ \\
\hline$C(41)$ & $27(1)$ & $46(2)$ & $26(1)$ & $-10(1)$ & $5(1)$ & $-16(1)$ \\
\hline$C(42)$ & $36(1)$ & $22(1)$ & $30(2)$ & $0(1)$ & $-8(1)$ & $-14(1)$ \\
\hline$C(43)$ & $41(2)$ & 28(1) & $37(2)$ & $-2(1)$ & $-11(1)$ & $-15(1)$ \\
\hline
\end{tabular}




\section{Computational Details}

\subsection{General}

All calculations were performed without symmetry restrictions. Starting coordinates for the corresponding structures were obtained from the crystal structure analyses or modelled with GaussView 5.0.9. The geometry optimization and the NBO analysis were performed with the Gaussian09 (Revision E.01) ${ }^{[1]}$. The geometry optimization was performed using Density-Functional Theory (DFT) ${ }^{[2]}$ with the PBE0 functional ${ }^{[3]}$ and the LANL2TZ(f) basis set and the corresponding ECP for Palladium ${ }^{[4]}$ and the def2svp basis set ${ }^{[5]}$ for all other atoms including Grimme's D3 dispersion correction with Becke-Johnson damping. ${ }^{[6]}$ The metrical parameters of the energy-optimized geometry compared well with those determined by X-ray diffraction. Harmonic vibrational frequency analysis was performed on the same levels of theory to determine the nature of the structure..$^{[7]}$ The vibrational frequency analysis showed no imaginary frequencies for minima and one imaginary frequency for transition states, with the displacement of the atoms corresponding to the expected motion during the transition state. Coordinates of all energy-optimized structures are provided as cartesian coordinates in Angstrom in the Tables below.

Energies: Single point energies were obtained with the PBE0 functional or the M06 ${ }^{[8]}$ functional and the LANL2TZ(f) basis set and the corresponding ECP for Palladium and the def2tzvp basis set for all other atoms with Grimme's D3 dispersion correction with Becke-Johnson damping. Solvent corrections were included by using the polarizable continuum model (PCM) for THF, which is also the solvent used in experiments. The energies were corrected by conversion from the standard state (1 atm) to the solution state $(1 \mathrm{M})$ state as follows: $\mathrm{E}^{\circ}{ }_{\mathrm{M}}=\mathrm{E}^{\circ}{ }_{\mathrm{Gas}}+\mathrm{RT} \ln \left(\mathrm{C}_{\mathrm{M}} / \mathrm{C}_{\mathrm{Gas}}\right)$, with $\mathrm{R}=8.31447 \mathrm{~J} \mathrm{~K}-1 \mathrm{~mol}-1, \mathrm{C}_{\mathrm{M}}=1 \mathrm{~mol} / \mathrm{L}$, $\mathrm{CGas}_{\mathrm{a}}=\mathrm{p} / \mathrm{RT}=0.0408 \mathrm{~mol} / \mathrm{L}$ (ideal Gas), $\mathrm{T}=$ temperature in $\mathrm{K}=298,15 \mathrm{~K}$. If the number of moles remains the same during the reaction, this correction cancels out. Only those reactions where the number of moles change are affected. For example, for a dissociation reaction such as $L_{2} P d \rightarrow L P d+L$ the free energy is corrected by $7.920 \mathrm{~kJ} / \mathrm{mol}$ (valid at $298 \mathrm{~K}$ ). Corrections for compounds in different concentrations were performed accordingly. To account for the concentration of the catalyst $(c=0,0005$ $\mathrm{mol} / \mathrm{L})$ versus that of the substrate $(1 \mathrm{~mol} / \mathrm{L})$ the correction term $\mathrm{RT}$ In $(0.0005 / 1)=-13.1 \mathrm{~kJ} / \mathrm{mol}$ was applied. Energies are provided in Hartree if not stated otherwise.

The NBO analyses ${ }^{[0]}$ were done with the B3LYP functional ${ }^{[10]}$ and the LANL2TZ(f) basis set and the corresponding ECP for Palladium and the $6-31+G^{*}$ basis set ${ }^{[11]}$ for all other atoms.

\subsection{NBO Studies}

Table S10. Comparison NBO analysis of the agostic C-H bond with the other Phosphonium group "ipso" $\mathrm{C}-\mathrm{H}$ bonds

\begin{tabular}{|c|c|c|c|}
\hline & $\mathrm{L} 1 \mathrm{Pd}(\mathrm{dba})$ & $\mathrm{L} 1{ }_{2} \mathrm{Pd}$ & \\
\hline charge $\mathrm{H} 28, \mathrm{H} 46 / \mathrm{H} 136$ respectively (agostic) & 0.2205 & 0.29245 & 0.29244 \\
\hline charge $\mathrm{H} 11, \mathrm{H} 12 / \mathrm{H} 29$ (“ipso”, non agostic) & 0.25914 & 0.25485 & 0.24882 \\
\hline charge H45, H102/H119 ("ipso", non agostic) & 0.25192 & 0.25485 & 0.24881 \\
\hline charge $\mathrm{C} 27, \mathrm{C} 45 / \mathrm{C} 135$ respectively (agostic) & -0.56766 & -0.62181 & -0.62183 \\
\hline charge C10, C11/C28 (“ipso”, non agostic) & -0.60566 & -0.60661 & -0.60705 \\
\hline charge C44, C101/C118(“ipso”, non agostic) & -0.60982 & -0.60658 & -0.60705 \\
\hline occupation $\mathrm{C} 27-\mathrm{H} 28, \mathrm{C} 45-\mathrm{H} 46 / \mathrm{C} 135-\mathrm{H} 136$ (agostic), & 1.92606 & 1.95331 & 1.95330 \\
\hline $\begin{array}{l}\text { occupation } \mathrm{C} 10-\mathrm{H} 11, \mathrm{C} 11-\mathrm{H} 12 / \mathrm{C} 28-\mathrm{H} 29 \text { (“ipso", non } \\
\text { agostic) }\end{array}$ & 1.96379 & 1.96406 & 1.96852 \\
\hline occupation C44-H45, C101-H102/C118-H119 & 1.96787 & 1.96406 & 1.96852 \\
\hline
\end{tabular}

("ipso", non agostic) 


\begin{tabular}{|c|c|c|c|}
\hline $\begin{array}{l}\text { occupation } \mathrm{C} 27-\mathrm{H} 28^{*}, \mathrm{C} 45-\mathrm{H} 46^{*} / \mathrm{C} 135-\mathrm{H} 136^{*} \\
\text { (agostic) }\end{array}$ & 0.05506 & 0.04671 & 0.04671 \\
\hline $\begin{array}{l}\text { occupation } \mathrm{C} 10-\mathrm{H} 11^{*}, \mathrm{C} 11-\mathrm{H} 12^{*} / \mathrm{C} 28-\mathrm{H} 29^{*} \text { ("ipso", } \\
\text { non agostic) }\end{array}$ & 0.02605 & 0.02603 & 0.02656 \\
\hline $\begin{array}{l}\text { occupation } \mathrm{C} 44-\mathrm{H} 45^{*}, \mathrm{C} 101-\mathrm{H} 102 * / \mathrm{C} 118-\mathrm{H} 119^{*} \\
\text { (“ipso", non agostic) }\end{array}$ & 0.0267 & 0.02604 & 0.02656 \\
\hline WBI C27-H28, C45-H46/C135-H136 (agostic) & 0.8297 & 0.8226 & 0.8226 \\
\hline $\begin{array}{l}\text { WBI C10-H11, C11-H12/C28-H29 (“ipso”, non } \\
\text { agostic) }\end{array}$ & 0.874 & 0.8764 & 0.8818 \\
\hline $\begin{array}{l}\text { WBI C44-H45, C101-H102/C118-H119 ("ipso", non } \\
\text { agostic) }\end{array}$ & 0.8797 & 0.8764 & 0.8818 \\
\hline WBI Pd1-H28, Pd1-H46/H136 & 0.055 & 0.0341 & 0.0341 \\
\hline WBI Pd1-C27, Pd1-C45/C135 & 0.0263 & 0.0244 & 0.0244 \\
\hline Exact electron density at BCP & 0.04362233993 & 0.01804247924 & 0.01803806051 \\
\hline
\end{tabular}

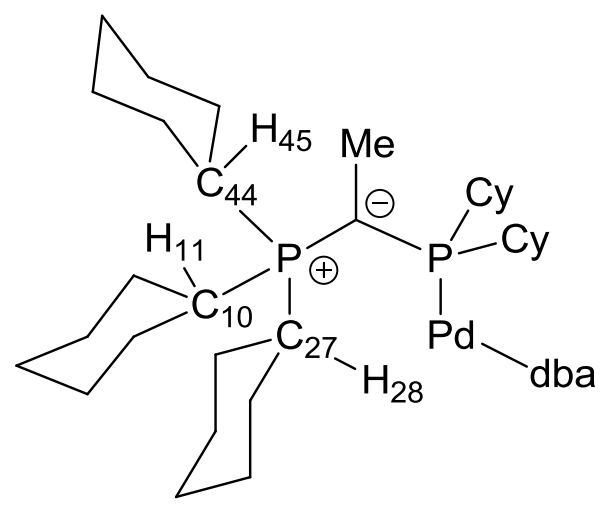

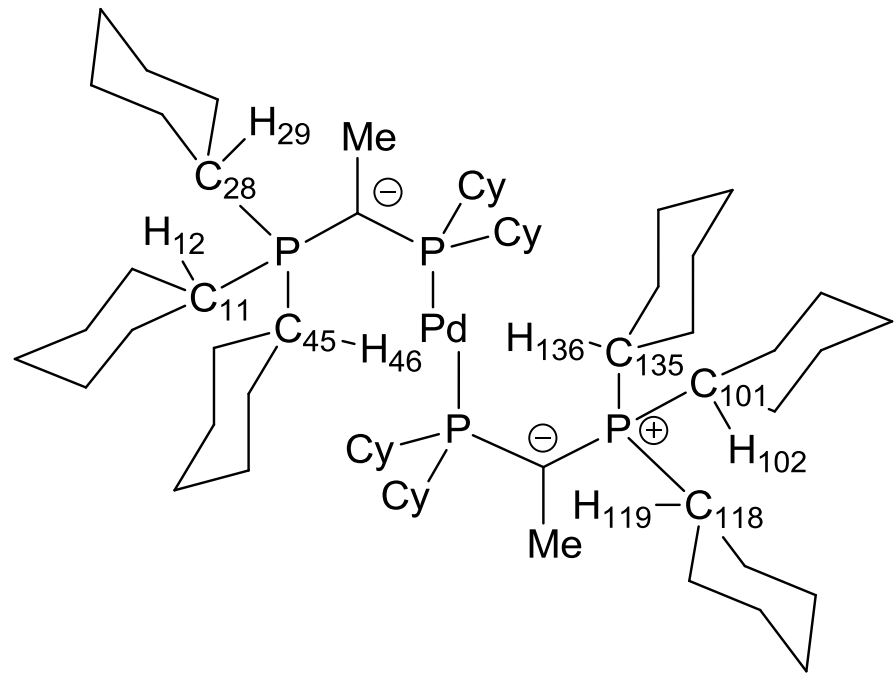

\subsection{Energies and Coordinates}

\subsubsection{Energies and Coordinates Related to Scheme 1}

Table S11. SCF energies and enthalpy/free energy corrections for Scheme 1. Relative energies are given relative to arene complex $1 . \Delta G_{M}$ is the solution state correct energy (with $1 \mathrm{~mol} / \mathrm{L}$ concentration for all reagents).

\begin{tabular}{|c|c|c|c|c|c|c|}
\hline PBE0-D3 & Corr(H) & Corr(G) & $\mathrm{E}(\mathrm{SCF})$ & $\mathrm{E}(\mathrm{PCM})$ & $\Delta G(P C M) / E_{H}$ & $\begin{array}{c}\Delta G_{M}(P C M) / k \\
\mathrm{~J} / \mathrm{mol}\end{array}$ \\
\hline L1 & 0.891335 & 0.786524 & -1935.860422 & -1935.863919 & & \\
\hline$L 1 \cdot P d(d b a)$ & 1.172695 & 1.026035 & -2793.558272 & -2793.570244 & -0.029489 & -77.424 \\
\hline $\mathrm{L1}_{2} \cdot \mathrm{Pd}$ & 1.787097 & 1.593734 & -3998.571174 & -3998.575968 & -0.043877 & -115.20 \\
\hline $\mathrm{L} 1 \cdot \mathrm{Pd}$ & 0.893958 & 0.784348 & -2062.621188 & -2062.629256 & 0.016054 & 50.057 \\
\hline L1·Pd·THF & 1.019484 & 0.895688 & -2294.92463 & -2294.92995 & 0.006373 & $16.731^{a}$ \\
\hline 1 & 0.993434 & 0.867313 & -2754.187606 & -2754.192664 & 0 & $0^{a}$ \\
\hline
\end{tabular}




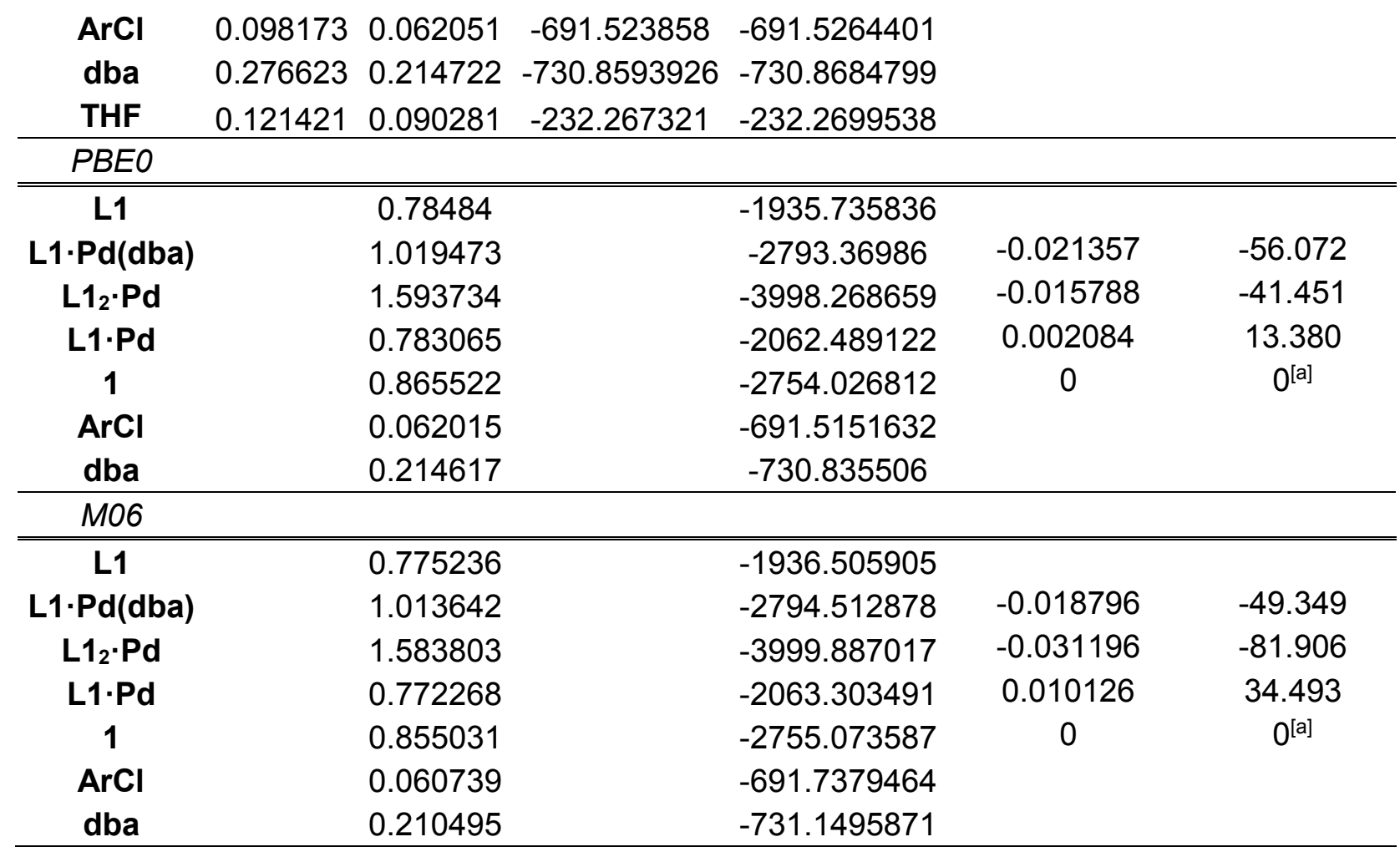

PBE0-D3 / def2tzvp + LANL2TZ(f) with PCM (THF)

[a] Correction of the energy due to the differences in the concentration lowers the energy of 1 by 18.8 $\mathrm{kJ} \cdot \mathrm{mol}^{-1}$, the energy of L1Pd(THF) $(\mathrm{c}=12 \mathrm{~mol} / \mathrm{L})$ by $25.0 \mathrm{~kJ} / \mathrm{mol}$.
L1
$E=-1935.86391942$
P $-1.2269690 .148476-0.118394$
P $1.702987-0.3486720 .328241$
C $0.331756-0.093477-0.787694$
C $0.456327-0.085182-2.299351$
H -0.449346 -0.464533-2.806305
H $1.278110-0.742228-2.630618$
H $0.6635420 .905440-2.744318$
C -1.886945 $1.892372-0.253499$
$\mathrm{H}-2.8375561 .9382730 .307518$
C -0.8801602 .8489640 .385316$
$\mathrm{H}-0.7054732 .5854281 .440751$
H $0.0870382 .703548-0.123752$
C -1.306180 4.307889 0.274260
H -2.220988 4.4722930.872429
H -0.528505 4.955125 0.711547
C -2.5953513.760952 -1.806973
$\mathrm{H}-2.7617744 .026579-2.863597$
$\mathrm{H}-3.5682403 .887241-1.297787$
C - $1.5738734 .696746-1.173652$
$\mathrm{H}-0.6286374 .641384-1.743527$
$\mathrm{H}-1.9162975 .742175-1.238154$
C -2.164802 2.300388 -1.703863
$\mathrm{H}-1.2513552 .146550-2.303510$
$\mathrm{H}-2.9429571 .659523-2.146883$
C -1.227069-0.215364 1.688926 
$\mathrm{H}-0.2577580 .2265181 .989041$

C - $1.118875-1.7113911 .992803$

$\mathrm{H}-2.063499-2.2139681 .721220$

$\mathrm{H}-0.320310-2.1556311 .381261$

C $-0.840366-1.9339863 .473932$

H $-0.773053-3.0133283 .686038$

H $0.149204-1.5050433 .711791$

C $-1.910193-1.2848594 .343647$

$\mathrm{H}-1.674368-1.4159925 .412181$

$\mathrm{H}-2.874906-1.7974814 .173851$

C -2.067533 0.195242 4.015790

$\mathrm{H}-1.1432600 .7296364 .299931$

$\mathrm{H}-2.8800470 .6393574 .613623$

C -2.330861 0.4307732 .529577

$\mathrm{H}-2.3950881 .5122342 .341383$

$\mathrm{H}-3.3133100 .0067422 .260494$

C - $2.515965-0.836962-1.019564$

$\mathrm{H}-2.528581-0.358449-2.016141$

C -3.931540 -0.730478 -0.449891

$\mathrm{H}-3.958952-1.2017160 .547915$

$\mathrm{H}-4.2244260 .321550-0.306845$

C $-4.937026-1.432327-1.359713$

H -5.945967-1.376854 -0.919529

H $-4.987636-0.891139-2.322043$

C $-4.545579-2.882957-1.618097$

H $-4.610181-3.448286-0.670515$

H -5.261947 -3.355432-2.309457

C -3.125434 -2.987381-2.163722

$\mathrm{H}-3.083087-2.520137-3.164597$

$\mathrm{H}-2.843382-4.043428-2.303354$

C $-2.121542-2.297261-1.246310$

H - $1.104649-2.349932-1.659771$

$\mathrm{H}-2.085452-2.829313-0.282870$

C 2.9307411 .0737100 .129465

H 3.9286660 .6073230 .228014

C $2.8831961 .804295-1.209459$

H $3.0656931 .099386-2.035984$

H $1.8609132 .194399-1.358395$

C $3.8754242 .962425-1.276067$

H $4.9055122 .563222-1.235802$

H $3.7844043 .486832-2.242146$

C $3.6734163 .934971-0.120067$

H $2.6769534 .406136-0.213943$

H $4.4095574 .754307-0.168887$

C 3.7606563 .2146011 .220507

H 3.5860803 .9194412 .050686

H 4.7873242 .8267061 .353673

C 2.7744632 .0533961 .295551

H 2.8838331 .5103442 .248423

H 1.7449102 .4526671 .292469

C $2.695437-1.718411-0.488467$

H $3.095227-1.352241-1.454947$

C $3.880519-2.1297070 .390158$

H $4.547201-1.2729320 .577553$

H $3.493138-2.4373041 .378923$

C $4.677806-3.276582-0.224985$ 
H $5.141981-2.930304-1.166488$ H $5.507455-3.5628490 .442617$ C $3.790413-4.480705-0.517687$ H $3.420677-4.8962160 .437612$ H $4.372423-5.282887-1.000802$ C $2.598579-4.086567-1.382445$ H $1.936962-4.953835-1.545117$ H $2.959937-3.780726-2.381379$ C $1.813545-2.934435-0.764024$ H $1.366250-3.2680310 .191389$ H 0.969835 -2.649365 -1.408999

\section{L1.Pd(dba)}

$E=-2793.57024434$

Pd $-0.0046171 .093387-0.953451$

P 0.900113 -2.337660 0.186868

P 0.7919340 .5523281 .159294

O -2.0840720.808356 -3.933869

C $1.171039-1.1588051 .417034$

C $1.528747-1.6479542 .810302$

H $0.875712-1.2034993 .581963$

H 2.566937 -1.415308 3.110789

H $1.413898-2.7364782 .929511$

C $2.052597-3.7563560 .481926$

H $1.696126-4.1624561 .446224$

C $1.973221-4.893008-0.539014$

H $2.338132-4.532069-1.515432$

H $0.933344-5.219085-0.693679$

C $2.829842-6.075754-0.093316$

H $2.402400-6.4989340 .833783$

H $2.784645-6.876600-0.848871$

C $4.274940-5.6616320 .160058$

H $4.735023-5.354736-0.796788$

H $4.861402-6.5208120 .523414$

C $4.357511-4.5013721 .145827$

H $5.403701-4.1811661 .276403$

H $4.009879-4.8379722 .139549$

C $3.505770-3.3202920 .692193$

H $3.556663-2.4953231 .415701$

H $3.910311-2.925895-0.253101$

C $1.100795-1.684836-1.524933$

H $0.469270-0.756583-1.481593$

C $0.528227-2.507366-2.683088$

$\mathrm{H}-0.484972-2.871804-2.467834$

H $1.156113-3.399726-2.844923$

C $0.505918-1.655323-3.951725$

H $0.119462-2.255012-4.791248$

H - $0.208934-0.823886-3.818875$

C $1.884857-1.100701-4.294054$

H $2.549704-1.933011-4.590744$

H $1.815101-0.433501-5.168089$

C $2.512114-0.367539-3.113930$

H $1.9328280 .547924-2.889051$

H $3.537114-0.042954-3.356056$

C $2.520184-1.235633-1.861877$

H $3.142086-2.129919-2.042902$ 
H $2.962255-0.698104-1.014929$

C $-0.785262-3.1304730 .163845$

H - $0.754757-3.951987-0.574709$

C -1.108890 -3.719562 1.540818

$\mathrm{H}-0.379931-4.5011571 .809832$

H - $1.004976-2.9208312 .295459$

C -2.520594 -4.293862 1.607662

$\mathrm{H}-2.719185-4.6696582 .624645$

$\mathrm{H}-2.589542-5.1673840 .934072$

C -3.563313 -3.262839 1.197545

H $-4.573924-3.7008961 .225343$

H -3.565390 -2.431365 1.925317

C -3.259601-2.711278 -0.187800

$\mathrm{H}-3.995464-1.943087-0.464085$

H -3.356764 -3.522599 -0.932688

C - $1.855842-2.121680-0.265004$

$\mathrm{H}-1.790717-1.2389970 .387907$

$\mathrm{H}-1.671201-1.748609-1.282918$

C - 0.3587591 .0807462 .535086

H 0.1456790 .8623713 .494297

C - 0.6538892 .5798922 .480275

$\mathrm{H}-1.0861562 .8082711 .488945$

H 0.2738583 .1676852 .552144

C - 1.6220833 .0090243 .579044

$\mathrm{H}-1.1376422 .8675004 .562262$

$\mathrm{H}-1.8365544 .0870783 .493022$

C -2.915198 2.2038513.538474

H -3.4558892.4414352.604186

$\mathrm{H}-3.5822592 .5008374 .364184$

C -2.632749 0.7069033 .587669

$\mathrm{H}-2.2057920 .4507364 .574304$

$\mathrm{H}-3.5698140 .1329073 .495098$

C - 1.6590430 .2860822 .493194

$\mathrm{H}-1.440588-0.7898642 .564033$

$\mathrm{H}-2.1288930 .4411171 .505107$

C 2.2809901 .6283731 .514201

H 1.9047322 .6430781 .284306

C 3.4012031 .3429490 .519033

H 3.7519330 .3052230 .668746

H $2.9991321 .404010-0.505699$

C 4.5668472 .3122250 .681684

H 4.2253473 .3242540 .402675

H $5.3751852 .051881-0.021760$

C 5.0892982 .3306172 .113089

H 5.8985003 .0717062 .219706

H 5.5346371 .3473052 .352256

C 3.9665272 .6204613 .102320

H 4.347325 2.6010194.136908

H 3.5852253 .6432292 .928164

C 2.8160831 .6288552 .945917

H 2.0176011 .8686643 .665537

H 3.1733460 .6175693 .202481

C -1.940657 1.464319-2.905941

C - $0.7049512 .212104-2.652065$

H -0.007086 2.195067 -3.494338

C $-0.4785183 .058082-1.542032$ 
H -1.330999 3.362394 -0.926000

C $0.7032713 .919860-1.369916$

C $0.7265804 .840586-0.309587$

H -0.147099 4.918866 0.342640

C $1.8411635 .640110-0.072675$

H 1.8345276 .3456360 .761824

C $2.9596765 .544374-0.898943$

H $3.8363046 .170141-0.716684$

C $2.9481264 .640879-1.964040$

H $3.8193484 .557858-2.618362$

C $1.8375343 .838610-2.196572$

H $1.8523893 .127848-3.025053$

C -2.973116 $1.428090-1.832256$

H -2.820675 $2.032508-0.936595$

C -4.045204 $0.624331-1.947337$

H -4.124479 $0.059537-2.883299$

C -5.082585 0.399673 -0.945309

C -5.007957 0.9278420 .355739

$\mathrm{H}-4.1601021 .5557420 .634909$

C -5.987160 0.6455261.299407

H -5.9035281 .0635612 .305759$

C -7.070208 -0.1720450 .967369$

H -7.837576 -0.396860 1.711763

C -7.163656 -0.699875 -0.319284

$\mathrm{H}-8.007404-1.339558-0.588940$

C -6.179715 -0.417730-1.263009

H -6.249298 -0.840636 -2.268671

\section{$\mathrm{L1}_{2} \cdot \mathrm{Pd}$}

$E=-3998.57596804$

Pd $-0.0000860 .000346-0.085552$

P $4.173422-0.171475-0.047406$

P 1.6550401 .6315880 .132407

$P-4.1735740 .171724-0.046947$

$\mathrm{P}-1.655156-1.6319380 .124694$

C 3.4142531 .3488680 .183070

C 4.3354852 .5034100 .542803

H 3.9742943 .0678441 .420387

H $4.4630943 .242226-0.270449$

H 5.3527392 .1774120 .808652

C $5.7781410 .090593-0.956718$

H $6.3840260 .635105-0.210167$

C $5.6515931 .020634-2.166205$

H $5.0658030 .527407-2.957238$

H $5.0919421 .923716-1.889063$

C $7.0287681 .382202-2.712617$

H $6.9234982 .035413-3.593930$

H $7.5763931 .970719-1.953900$

C $7.8360460 .138001-3.065424$

H $8.8411570 .415811-3.421925$

H $7.342838-0.385739-3.904491$

C $7.938349-0.811966-1.877625$

H $8.533658-0.334334-1.078240$

H 8.480025 -1.728799 -2.161876

C $6.561715-1.171700-1.323135$

H $6.005047-1.739143-2.088155$ 
H $6.671051-1.839383-0.454519$

C $4.748648-1.0418401 .504414$

H $5.302967-1.9423531 .182300$

C $3.555232-1.4769852 .356026$

H $2.945387-0.5896582 .581909$

H $2.897385-2.1501951 .790823$

C $3.991626-2.1526193 .652214$

H $4.498909-3.1057863 .414320$

H $3.105505-2.4147294 .250458$

C $4.934128-1.2710474 .459476$

H $5.264445-1.7909935 .373320$

H $4.389613-0.3686044 .791915$

C $6.131884-0.8506833 .619518$

H $6.793678-0.1808254 .192427$

H $6.733345-1.7433833 .368599$

C $5.692613-0.1624362 .331182$

H 6.5822670 .1205141 .746701

H 5.1684460 .7748572 .584949

C $3.116972-1.397394-0.918372$

H $2.125297-1.251722-0.425382$

C $3.547262-2.862205-0.778462$

H $4.532787-3.008766-1.250432$

H $3.661795-3.1513960 .275064$

C $2.536576-3.791442-1.444036$

H $2.885135-4.834286-1.368147$

H $1.588932-3.739752-0.882649$

C $2.278820-3.417803-2.897625$

H $3.196513-3.596740-3.487814$

H $1.504805-4.074774-3.325887$

C $1.878908-1.953537-3.027813$

H $0.915231-1.771456-2.520102$

H $1.734852-1.683375-4.086649$

C $2.923258-1.045578-2.394685$

H $2.6291660 .006552-2.490108$

H $3.879147-1.168477-2.933135$

C $1.3667083 .009886-1.126722$

H $0.2666083 .033474-1.211377$

C $1.9134762 .578247-2.484772$

H $1.4794901 .600959-2.751507$

H $3.0013102 .416474-2.390194$

C $1.6334743 .588671-3.592278$

H $0.5498203 .604865-3.801228$

H $2.1212513 .265427-4.526977$

C $2.0854204 .992497-3.210673$

H $3.1873325 .010108-3.121657$

H $1.8261635 .711373-4.005458$

C $1.4746765 .416633-1.880631$

H $1.8128986 .429259-1.604844$

H $0.3755085 .473982-1.986162$

C $1.8223554 .426936-0.771478$

H 1.3650634 .7561000 .174113

H $2.9110194 .445003-0.609976$

C 1.2824342 .5384671 .730324

H 1.9168203 .4417461 .779175

C 1.6246351 .6549952 .925699

H 1.0862750 .6954792 .809518 
H 2.6986511 .4153202 .904459

C 1.2490342 .2967744 .255923

H 1.8730373 .1945894 .419189

H 1.4757531 .6057145 .085252

C - 0.2203942 .6989084 .281739

$\mathrm{H}-0.8415161 .7870524 .212486$

$\mathrm{H}-0.4767803 .1821175 .239127$

C - 0.5500483 .6157103 .110312

H 0.0062014 .5642143 .224962

$\mathrm{H}-1.6194163 .8846293 .116986$

C - 0.1812462 .9687231 .780242

$\mathrm{H}-0.4131583 .6570490 .952232$

$\mathrm{H}-0.7956312 .0647461 .611690$

C -3.414446 -1.349747 0.176015

C -4.335365 -2.506679 0.528622

H -3.974539-3.075765 1.403346

$\mathrm{H}-4.461822-3.241037-0.288848$

H $-5.353016-2.1827180 .795412$

C $-5.778481-0.085895-0.957137$

H $-6.384038-0.634375-0.213220$

C $-5.652305-1.009532-2.171578$

H -5.092315 -1.913970 -1.899599

H -5.067043 -0.511928 -2.960272

C -7.029702 -1.368480 -2.719174

$\mathrm{H}-6.924786-2.017167-3.603867$

H -7.576832 -1.960961-1.963189

C -7.837351 -0.122634 -3.065226

$\mathrm{H}-8.842618-0.398821-3.422552$

H -7.344716 $0.405405-3.901929$

C $-7.9391170 .821301-1.872590$

$\mathrm{H}-8.5341070 .339660-1.075376$

$\mathrm{H}-8.4808671 .739590-2.151958$

C -6.562236 1.178185-1.316839

$\mathrm{H}-6.6713061 .841518-0.444883$

H -6.005828 $1.749472-2.079182$

C -4.7485281 .0343421 .509262$

H -5.303296 1.9362371 .191832

C -5.691846 0.1501972.331708

H -6.581560 -0.130036 1.746012

H -5.167137 -0.788223 2.580182

C -6.131020 0.831307 3.623840

H -6.733169 1.7249263 .377898

H -6.792146 0.157977 4.193439

C -4.933234 1.2480334 .465554

$\mathrm{H}-4.3879960 .3442354 .793091$

$\mathrm{H}-5.2635601 .7630025 .382208$

C -3.9916142.134422 3.662540

H -4.499689 3.0884073 .429668

$\mathrm{H}-3.1055202 .3941554 .261858$

C -3.555118 1.4657362 .362788

$\mathrm{H}-2.9447400 .5775912 .584016$

H -2.897819 2.142217 1.800868

C -3.117217 $1.401851-0.912063$

$\mathrm{H}-2.1256561 .253972-0.419577$

C -2.922947 $1.056903-2.389919$

$\mathrm{H}-3.8786381 .182085-2.928181$ 
H -2.628554 $0.005269-2.489962$

C -1.878475 $1.967919-3.018413$

$\mathrm{H}-0.9149611 .783436-2.511271$

H - $1.7340791 .702785-4.078473$

C $-2.2783683 .431564-2.881390$

H -3.195900 $3.613380-3.470952$

$\mathrm{H}-1.5041944 .090503-3.306324$

C -2.536434 $3.798265-1.426087$

$\mathrm{H}-2.8848104 .840797-1.345251$

$\mathrm{H}-1.5889423 .743624-0.864721$

C -3.547524 2.865981-0.765370

$\mathrm{H}-3.6626103 .1501630 .289477$

$\mathrm{H}-4.5327683 .014915-1.237177$

C -1.366463 -3.004308-1.140847

H -0.266397 -3.026869-1.226016

C - $1.913945-2.566744-2.496716$

$\mathrm{H}-3.001844-2.406069-2.401055$

H - $1.480749-1.587919-2.759067$

C -1.633633 -3.571755 -3.609065

$\mathrm{H}-0.550060-3.586089-3.818601$

$\mathrm{H}-2.122117-3.244497-4.541990$

C - $2.084257-4.977732-3.233843$

$\mathrm{H}-3.186120-4.996707-3.144502$

H -1.824679 -5.692654 -4.032082

C - $1.472663-5.407517-1.906022$

$\mathrm{H}-0.373480-5.463290-2.012178$

$\mathrm{H}-1.809791-6.421758-1.634861$

C $-1.820949-4.423378-0.792124$

$\mathrm{H}-1.363202-4.7566370 .151809$

H -2.909579 -4.443167 -0.630535

C - $1.282704-2.5461881 .718458$

$\mathrm{H}-1.916927-3.4498031 .763062$

C $0.181088-2.9763271 .766622$

H $0.795227-2.0713221 .602768$

H $0.413504-3.6603630 .935214$

C $0.549700-3.6298103 .093566$

H -0.006526 -4.578889 3.203494

H $1.619071-3.8987193 .099141$

C $0.219796-2.7187064 .269362$

H $0.840987-1.8065574 .204689$

H $0.475919-3.2065535 .224467$

C - $1.249620-2.3164444 .245181$

$\mathrm{H}-1.873630-3.2150304 .404100$

$\mathrm{H}-1.476479-1.6293235 .077741$

C -1.625089-1.668399 2.917960

$\mathrm{H}-1.086720-0.7083382 .806358$

H -2.699112 -1.428650 2.897826

\section{L1·Pd}

$E=-2062.62925636$

Pd -1.565936 -0.105541 1.990268

P $1.5531910 .317750-0.247434$

$\mathrm{P}-1.478217-0.229922-0.238685$

C $0.0690220 .054496-1.073580$

C $0.0152890 .282955-2.572283$

H - $0.551813-0.512844-3.089834$ 
H $1.0106920 .295703-3.042986$

$\mathrm{H}-0.4632511 .238345-2.862012$

C $2.954017-0.209124-1.339899$

H $2.8962250 .496609-2.188633$

C $4.344106-0.051862-0.719881$

H $4.443540-0.7460950 .131883$

H $4.4875030 .962219-0.316327$

C $5.431600-0.361487-1.745787$

H $5.3947420 .398356-2.547451$

H $6.425381-0.270413-1.278169$

C $5.253416-1.746961-2.356141$

H $5.410835-2.509528-1.571778$

H 6.022668 -1.929371 -3.123809

C $3.857361-1.919912-2.944129$

H $3.728165-2.941847-3.335471$

H $3.736197-1.239849-3.807078$

C $2.775150-1.617499-1.913130$

H $1.772363-1.727193-2.348280$

H $2.835401-2.355342-1.097824$

C $1.610191-0.5284641 .378200$

H $0.598555-0.2285261 .769341$

C $2.661266-0.0736242 .391332$

H 2.7204161 .0215242 .458496

H $3.662351-0.4218252 .083387$

C $2.328774-0.6523393 .765773$

H $3.089665-0.3413934 .500106$

H $1.369558-0.2201464 .104979$

C $2.211824-2.1722863 .731602$

H $3.207365-2.6037643 .518845$

H $1.915367-2.5547894 .721842$

C $1.230375-2.6359742 .661780$

H $0.205681-2.3057372 .919113$

H $1.205012-3.7366562 .611330$

C $1.579336-2.0526741 .298130$

H $2.566338-2.4326530 .978926$

H $0.848998-2.3711420 .543216$

C 1.9590252 .0955480 .150932

H 2.9673762 .1279550 .602100

C $1.9691202 .930859-1.133377$

H $2.7614422 .582558-1.815216$

H $1.0124452 .776626-1.661670$

C $2.1544234 .418771-0.853960$

H $2.1211134 .981852-1.800834$

H $3.1600844 .586687-0.427118$

C 1.1047414 .9390850 .118971

H 1.2653726 .0100480 .323182

H $0.1050744 .854518-0.344227$

C 1.1274774 .1373561 .413450

H 0.3430174 .4909332 .102064

H 2.0917564 .3073531 .927049

C 0.9440072 .6458111 .156711

H -0.068822 2.453949 0.771198

H 0.9997242 .0956012 .107674

C -2.654212 0.990571-1.046526

$\mathrm{H}-2.5944850 .799915-2.134913$

C $-4.1071730 .799862-0.613455$ 
$\mathrm{H}-4.1541530 .8745850 .489083$

H $-4.458736-0.210289-0.871784$

C -5.034445 $1.833740-1.248320$

$\mathrm{H}-5.0690501 .666316-2.340510$

H -6.063918 $1.688954-0.881053$

C -4.567568 $3.258924-0.979095$

$\mathrm{H}-4.6360273 .4638480 .104793$

H -5.231880 $3.984482-1.476729$

C $-3.1252473 .450986-1.430841$

$\mathrm{H}-3.0709073 .349591-2.530330$

$\mathrm{H}-2.7775314 .471133-1.195984$

C -2.203906 2.423546 -0.784164

$\mathrm{H}-1.1691402 .556887-1.134013$

$\mathrm{H}-2.1852952 .5812710 .310179$

C $-2.185000-1.837365-0.939185$

C - $1.122781-2.848718-1.359532$

C -3.152485 -2.501479 0.043957

H $-2.751859-1.548823-1.845789$

C $-1.752469-4.091884-1.980733$

$\mathrm{H}-0.549051-3.150799-0.466638$

H $-0.396857-2.390173-2.045816$

C -3.795996 $-3.750250-0.551742$

$\mathrm{H}-2.582583-2.7635890 .954304$

$\mathrm{H}-3.923152-1.7923270 .380100$

C -2.747078 -4.747996-1.029504

$\mathrm{H}-0.967491-4.810135-2.271101$

H -2.272280 -3.806606 -2.913739

$\mathrm{H}-4.465446-4.2207980 .187319$

H -4.434744 -3.456823 -1.405183

H -3.227331 -5.616892 -1.509098

H -2.199133 $-5.139675-0.153070$

\section{L1·Pd·THF}

$E=-2294.92995013$

$\mathrm{Pd}-1.632334-0.1241871 .160624$

P $1.8639150 .487473-0.296584$

P $-0.791841-1.000580-0.698916$

C $0.835243-0.512023-1.241585$

C $1.221417-0.821689-2.674382$

H 1.050050 -1.885054 -2.924992

H $2.284820-0.630632-2.888287$

H $0.656708-0.236227-3.425899$

C $3.6272150 .138941-0.747694$

H $3.6930670 .467994-1.800926$

C 4.6631590 .9437690 .039995

H 4.6320680 .6379681 .099622

H 4.4326092 .0199560 .017807

C $6.0673340 .701946-0.508605$

H $6.1241691 .102500-1.537102$

H 6.8047221 .2665020 .084908

C $6.418720-0.781353-0.525647$

H $6.479425-1.1493130 .514751$

H $7.417146-0.933714-0.966577$

C $5.370024-1.592343-1.278766$

H $5.612053-2.666622-1.238257$

H $5.386869-1.310734-2.347579$ 
C $3.971305-1.352879-0.720473$

H $3.218808-1.927823-1.277377$

H $3.927662-1.7199920 .316954$

C 1.5744530 .2893651 .502355

H 0.4487460 .3453901 .507828

C 2.1068211 .3696432 .444546

H 1.8984032 .3819342 .070687

H 3.2044661 .2919922 .533592

C 1.4784801 .1939563 .826578

H 1.8634831 .9626944 .516640

H 0.3893651 .3583773 .742288

C $1.725969-0.2007324 .390803$

H $2.806551-0.3197974 .593647$

H $1.214348-0.3151895 .360312$

C $1.276781-1.2842833 .417765$

H $0.178863-1.2440713 .290511$

H $1.517059-2.2837813 .815554$

C $1.914296-1.0959562 .046493$

H $3.010266-1.2094442 .131423$

H 1.561424 -1.865530 1.348223

C $1.7065842 .328046-0.583963$

H 2.4840782 .8406980 .011099

C $1.9314392 .645469-2.065537$

H $2.9571742 .378920-2.366797$

H $1.2554302 .013255-2.666663$

C $1.6690564 .112462-2.390504$

H $1.8091984 .285395-3.469964$

H $2.4187604 .739988-1.874832$

C $0.2739664 .540393-1.954561$

H $0.1084995 .606110-2.181409$

H $-0.4779973 .975243-2.534336$

C $0.0694464 .269985-0.469957$

$\mathrm{H}-0.9530644 .546386-0.163489$

H 0.7529314 .9149350 .112654

C $0.3285632 .808605-0.122676$

H -0.438112 2.168556 -0.585566

H 0.2118912 .6568860 .960122

C -1.889890 -0.579346 -2.167358

$\mathrm{H}-1.443217-1.074274-3.050309$

C -3.318612 -1.097084-2.005779

H -3.720163 $-0.705157-1.052109$

H -3.324412 -2.193727 -1.916528

C $-4.221084-0.679804-3.164242$

$\mathrm{H}-3.875841-1.175298-4.090154$

H -5.248845 -1.038372 -2.987380

C -4.210999 $0.828711-3.376902$

$\mathrm{H}-4.6518631 .322663-2.491178$

$\mathrm{H}-4.8453001 .104241-4.235543$

C -2.787771 $1.336309-3.571499$

$\mathrm{H}-2.3791670 .921618-4.511230$

H -2.779021 $2.433450-3.687376$

C - $1.8945810 .926456-2.407165$

$\mathrm{H}-0.8643341 .279580-2.564475$

H -2.250567 $1.412573-1.479378$

C $-0.853102-2.886558-0.819503$

$\mathrm{H}-1.149147-3.120709-1.860733$ 
C -1.909297 -3.466169 0.125543

$\mathrm{H}-1.618035-3.2065761 .159620$

$\mathrm{H}-2.884415-2.980878-0.027677$

C - $2.033874-4.979512-0.024025$

$\mathrm{H}-2.415839-5.213771-1.034928$

H $-2.780603-5.3711980 .686718$

C -0.692646 -5.6753200 .178627$

$\mathrm{H}-0.788377-6.7608330 .010180$

H - $0.379339-5.5499971 .231226$

C $0.380286-5.083829-0.729108$

H $1.356006-5.557794-0.529341$

H $0.134379-5.311966-1.782538$

C $0.484877-3.571468-0.556413$

H $1.270660-3.154486-1.202332$

H $0.795987-3.3551970 .479906$

C -4.144724 0.8063512 .601004

O -2.742990 0.9555032 .804451

C -2.516864 2.357458 2.741617

C -3.360824 2.840262 1.563092

C -4.493774 1.7949091 .485518

$\mathrm{H}-4.338112-0.2456672 .356309$

H -4.666399 1.0626853 .542636

$\mathrm{H}-2.8523472 .8147773 .692097$

$\mathrm{H}-1.4379032 .5227552 .628895$

$\mathrm{H}-3.7275843 .8656141 .715241$

$\mathrm{H}-2.7599212 .8207990 .642390$

H -5.491627 2.2330761.631718

H -4.480745 1.2873900 .510317

1

$E=-2754.19266421$

$\mathrm{Pd}-1.6334060 .1873740 .778927$

P $2.1172470 .206936-0.118136$

P -0.651592 -0.441740 -1.226551

C $1.102528-0.258702-1.426757$

C $1.662723-0.283858-2.835197$

H $1.308709-1.165846-3.399558$

H $2.762421-0.331211-2.864960$

H $1.3842900 .599544-3.441973$

C $3.828583-0.416872-0.449984$

H $4.1161570 .132882-1.365103$

C $4.870197-0.0782690 .618326$

H $4.627882-0.6190801 .548755$

H 4.8538680 .9942320 .865465

C $6.267004-0.4848160 .154873$

H $6.5481240 .130886-0.718781$

H $7.003908-0.2593150 .942640$

C $6.327997-1.959570-0.226614$

H $6.166392-2.5729010 .678489$

H $7.332266-2.217588-0.599647$

C $5.264977-2.311826-1.261421$

H $5.288748-3.390334-1.485975$

H $5.490480-1.792495-2.210778$

C $3.871637-1.909357-0.790178$

H $3.110844-2.151786-1.545178$

H 3.609752 -2.495390 0.104917 
C $1.483069-0.3739251 .507633$

H $0.422094-0.0180051 .442601$

C 2.0929210 .2472312 .765965

H 2.163017 1.341045 2.688761

H $3.122160-0.1249202 .905745$

C $1.249361-0.1227493 .984704$

H 1.7043000 .3011614 .894729

H 0.2604920 .3553083 .880205

C $1.081292-1.6312974 .130844$

H $2.059384-2.0814434 .382410$

H $0.412218-1.8587394 .977168$

C $0.556335-2.2686762 .849422$

$\mathrm{H}-0.475706-1.9258312 .653160$

H $0.511176-3.3649552 .954165$

C $1.418155-1.8922711 .651176$

H $2.437173-2.2939521 .792049$

H $1.021814-2.3377190 .730002$

C 2.3532692 .0408430 .125017

H 3.1072802 .1901250 .918955

C $2.8703782 .675116-1.169861$

H $3.8643602 .274586-1.427030$

H $2.1959552 .389169-1.995240$

C $2.9388244 .196430-1.081077$

H $3.2803434 .609480-2.044159$

H $3.6992434 .483636-0.332100$

C $1.5953714 .794741-0.683979$

H $1.6697115 .891703-0.609131$

H $0.8543164 .583958-1.476114$

C 1.1096974 .1986090 .630081

H 0.1202534 .6002590 .901786

H 1.7995624 .4987531 .440295

C 1.0331202 .6779170 .567085

H $0.2431452 .375279-0.137975$

H 0.7126992 .2882391 .543492

C -1.395883 $0.570094-2.619105$

$\mathrm{H}-0.9286250 .208247-3.554646$

C -2.908530 $0.386872-2.741669$

$\mathrm{H}-3.3696880 .637950-1.769050$

H -3.157459 -0.666747 -2.939869

C -3.503488 $1.263432-3.840971$

$\mathrm{H}-3.1218910 .924310-4.821393$

H -4.597854 $1.132396-3.873690$

C $-3.1475362 .732732-3.650627$

$\mathrm{H}-3.6190013 .102521-2.721591$

H -3.559678 $3.340822-4.472537$

C -1.638443 2.916169-3.548730

$\mathrm{H}-1.1723722 .645514-4.513816$

$\mathrm{H}-1.3872863 .974959-3.368872$

C -1.051984 2.045592-2.444096

H $0.0400472 .169027-2.390633$

H -1.449048 2.375840 -1.466141

C - $1.137755-2.168368-1.814525$

$\mathrm{H}-1.380630-2.070899-2.890110$

C $-2.390707-2.651032-1.077441$

$\mathrm{H}-2.153392-2.7139170 .000552$

H -3.198861 -1.907580 -1.146832 
$\begin{array}{lll}\text { C }-2.861955 & -4.008080 & -1.589782 \\ \text { H }-3.194247 & -3.904286 & -2.639224 \\ \text { H }-3.743495 & -4.342672 & -1.018092 \\ \text { C }-1.752405 & -5.050985 & -1.515997 \\ \text { H }-2.091887 & -6.010093 & -1.940704 \\ \text { H }-1.514580 & -5.247472 & -0.454507 \\ \text { C }-0.490752 & -4.568522 & -2.223870 \\ \text { H } 0.317503 & -5.311092 & -2.116712 \\ \text { H }-0.690632 & -4.481663 & -3.307613 \\ \text { C }-0.033938 & -3.214294 & -1.689025 \\ \text { H } 0.881484 & -2.879483 & -2.198038 \\ \text { H } 0.234397 & -3.328157 & -0.625426 \\ \text { Cl }-1.815353 & 2.187397 & 3.174222 \\ \text { C }-2.737642 & 0.867064 & 2.452971 \\ \text { C }-3.590482 & 1.132749 & 1.343927 \\ \text { C }-2.890379 & -0.338434 & 3.187925 \\ \text { C }-4.624217 & 0.214190 & 1.049078 \\ \text { H }-3.586616 & 2.120806 & 0.879075 \\ \text { C }-3.900108 & -1.227760 & 2.848351 \\ \text { H }-2.238530 & -0.525822 & 4.043150 \\ \text { C }-4.785133 & -0.942808 & 1.794471 \\ \text { H }-5.309068 & 0.442851 & 0.228916 \\ \text { H }-4.009023 & -2.151891 & 3.420547 \\ \text { H }-5.592143 & -1.638942 & 1.557369\end{array}$

\section{Chlorobenzene}

$E=-691.526440085$

C $0.512722-0.000053-0.000004$

C $-0.1727701 .213851-0.000022$

C - 1.5663471 .2057410 .000024

C $-2.2665430 .000009-0.000001$

C $-1.566437-1.205686-0.000020$

C $-0.172786-1.2138660 .000016$

H $0.3859812 .150904-0.000026$

H -2.107780 2.1545930.000022

$\mathrm{H}-3.3585740 .0001080 .000005$

H -2.107801 -2.154578 -0.000011

H $0.385802-2.1510130 .000028$

Cl 2.2467850.0000010.000002

Dibenzylideneacetone (dba)

$\mathrm{E}=-730.868479885$

O $0.000002-2.112407-0.000166$

C $-0.000013-0.891837-0.000077$

C $1.255823-0.106548-0.000037$

H 1.1762880 .9842570 .000042

C $2.447221-0.733573-0.000075$

H $2.401035-1.829185-0.000117$

C $3.777140-0.136544-0.000040$

C $4.897631-0.9837430 .000234$

H $4.741916-2.0656960 .000397$

C $6.189406-0.4659260 .000308$

H $7.047136-1.1424660 .000530$

C 6.3859370 .9135800 .000093

H 7.3983341 .3243040 .000144

C $5.2824921 .770582-0.000200$ 
$\begin{array}{lll}\text { H } 5.432261 & 2.852815 & -0.000386 \\ \text { C } 3.993905 & 1.253127 & -0.000267 \\ \text { H } 3.141215 & 1.935292 & -0.000520 \\ \text { C }-1.255826 & -0.106532 & -0.000012 \\ \text { H }-1.176216 & 0.984266 & 0.000069 \\ \text { C }-2.447215 & -0.733556 & -0.000053 \\ \text { H }-2.401052 & -1.829170 & -0.000135 \\ \text { C }-3.777150 & -0.136539 & -0.000000 \\ \text { C }-3.993910 & 1.253133 & 0.000106 \\ \text { H }-3.141202 & 1.935277 & 0.000152 \\ \text { C }-5.282494 & 1.770584 & 0.000153 \\ \text { H }-5.432272 & 2.852817 & 0.000236 \\ \text { C }-6.385937 & 0.913574 & 0.000096 \\ \text { H }-7.398335 & 1.324294 & 0.000133 \\ \text { C }-6.189403 & -0.465931 & -0.000010 \\ \text { H }-7.047131 & -1.142473 & -0.000055 \\ \text { C }-4.897623 & -0.983748 & -0.000057 \\ \text { H }-4.741909 & -2.065701 & -0.000140\end{array}$

M06 / def2tzvp + LANL2TZ(f) with PCM (THF)

\section{L1}

$E=-1936.50590510$

P -1.237832 $0.124464-0.105757$

P $1.715507-0.3480690 .317616$

C $0.316042-0.140015-0.790420$

C $0.428695-0.140887-2.303675$

$\mathrm{H}-0.457779-0.570321-2.811181$

H $1.279103-0.764328-2.636146$

H $0.5905040 .852169-2.769333$

C $-1.8541591 .893799-0.162786$

$\mathrm{H}-2.8390031 .9342900 .346135$

C - 0.8773512 .8018760 .582792

H -0.745679 2.475118 1.630317

H 0.1158662 .6920120 .107032

C -1.298450 4.263451 0.537793

$\mathrm{H}-2.2563934 .3883521 .081378$

$\mathrm{H}-0.5576514 .8842361 .071082$

C - $2.4579963 .853817-1.638961$

$\mathrm{H}-2.5731374 .185620-2.685139$

$\mathrm{H}-3.4574903 .954805-1.170947$

C -1.468490 4.737574 -0.896562

$\mathrm{H}-0.4872994 .693452-1.410389$

$\mathrm{H}-1.7872955 .793465-0.927107$

C -2.036539 2.390356-1.599197

$\mathrm{H}-1.0779662 .271488-2.142009$

H -2.779220 $1.777335-2.140148$

C -1.271374 -0.311321 1.692491

$\mathrm{H}-0.3228700 .1464452 .043174$

C -1.141336 -1.812720 1.954749

$\mathrm{H}-2.084801-2.3188811 .668913$

$\mathrm{H}-0.341294-2.2434451 .330183$

C $-0.870604-2.0685793 .429866$

$\mathrm{H}-0.766905-3.1510243 .617017$

H $0.104213-1.6124543 .692370$

C -1.970993 -1.476566 4.297806 
H -1.757931 -1.638969 5.368291

$\mathrm{H}-2.920440-2.0088844 .088523$

C -2.160986 0.0054094 .011218

$\mathrm{H}-1.2498670 .5552944 .319187$

$\mathrm{H}-2.9882590 .4170444 .614740$

C -2.411579 0.271165 2.530366

$\mathrm{H}-2.5262711 .3557452 .367962$

$\mathrm{H}-3.373616-0.1906392 .233743$

C -2.549182 -0.789017-1.057671

H -2.498532 -0.298658 -2.051856

C -3.983173 $-0.625741-0.554266$

$\mathrm{H}-4.088968-1.1428280 .420066$

$\mathrm{H}-4.2327150 .434123-0.371619$

C -4.968917 -1.231728 -1.546929

H -6.001947 -1.123043-1.174143

H -4.920161 -0.656809-2.493258

C $-4.649313-2.692823-1.828465$

H $-4.799972-3.278929-0.900132$

$\mathrm{H}-5.353017-3.107381-2.570437$

C $-3.209868-2.865418-2.292608$

$\mathrm{H}-3.076105-2.363812-3.271985$

H -2.980664 -3.931591 -2.460244

C -2.232109-2.266383-1.290706

$\mathrm{H}-1.189107-2.388262-1.625260$

$\mathrm{H}-2.312062-2.817361-0.335453$

C 2.9322241 .0901220 .041136

H 3.9379530 .6211450 .009306

C $2.7571071 .878780-1.252241$

H $2.8564811 .210163-2.125198$

H $1.7234462 .279769-1.292430$

C $3.7422183 .037035-1.361319$

H $4.7728572 .634747-1.428516$

H $3.5710123 .600493-2.295715$

C $3.6495853 .955574-0.152713$

H $2.6373884 .409229-0.118697$

H $4.3605554 .795574-0.241290$

C 3.8872033 .1760691 .131462

H 3.8192613 .8387012 .012366

H 4.9218372 .7782961 .123478

C 2.9084602 .0169241 .257692

H 3.1090421 .4306362 .172620

H 1.8830142 .4211921 .384078

C $2.729135-1.721043-0.486855$

H $3.146433-1.353490-1.450658$

C $3.899344-2.1077060 .420017$

H $4.536165-1.2337680 .645361$

H $3.487187-2.4399321 .395038$

C $4.740132-3.227331-0.179841$

H $5.205159-2.868533-1.119572$

H 5.573464 -3.487937 0.496177

C $3.888758-4.451790-0.479957$

H $3.504707-4.8655610 .473834$

H $4.496976-5.250275-0.939810$

C $2.710851-4.087706-1.371516$

H $2.076917-4.972226-1.559807$

H $3.091298-3.765334-2.361432$ 
C $1.883806-2.958815-0.769374$

H $1.437523-3.3045120 .187348$

H $1.034082-2.705860-1.425665$

\section{L1.Pd(dba)}

$E=-2794.51287845$

Pd $0.266512-1.2048310 .945712$

P $0.2539222 .513611-0.145879$

P $0.921841-0.308675-1.158836$

O -1.820560 -1.651130 3.985006

C $0.8712001 .459272-1.371524$

C $1.2184122 .049161-2.727829$

H $0.7948981 .454651-3.560125$

H $2.3086632 .113818-2.924205$

H $0.8255473 .069457-2.882918$

C $1.0004124 .197926-0.384723$

H $0.6138794 .497915-1.380059$

C 0.5467045 .2690970 .608203

H 0.9402295 .0255721 .614509

$\mathrm{H}-0.5526245 .2954210 .701641$

C 1.0659486 .6400410 .190294

H $0.6024916 .918317-0.776995$

H 0.7431577 .4051110 .916864

C 2.5800276 .6425370 .041414

H 3.0400746 .4634441 .033397

H $2.9358977 .633726-0.287560$

C $3.0396155 .556145-0.919799$

H $4.1401785 .543056-0.995653$

H $2.6632315 .781273-1.937634$

C $2.5271904 .187559-0.491463$

H $2.8651753 .403117-1.186813$

H 2.9605893 .9339710 .493064

C 0.5506311 .9027051 .575378

H 0.2033620 .8412511 .487467

C -0.2714942.513132 2.713722

$\mathrm{H}-1.3373542 .6125962 .455272$

H 0.0945923 .5363642 .928584

C - 0.1275211 .6451403 .960418

$\mathrm{H}-0.7173932 .0740754 .787971$

H -0.569238 0.647989 3.759836

C 1.3291181 .4881014 .373432

H 1.7189982 .4720764 .703998

H 1.4089580 .8169125 .245414

C 2.1900070 .9810163 .225357

H $1.896892-0.0578552 .965329$

H 3.2528890 .9412593 .520839

C 2.0213181 .8485291 .985424

H 2.3673142 .8758792 .216367

H 2.646004 1.4794001 .157824

C -1.585732 $2.846810-0.176970$

$\mathrm{H}-1.7890683 .6169810 .594910$

C -2.016668 $3.404964-1.535396$

$\mathrm{H}-1.4785634 .342900-1.764092$

$\mathrm{H}-1.7279282 .680667-2.322743$

C -3.519005 $3.648790-1.602436$

H -3.794341 4.024653 -2.602942 
H -3.787278 $4.450988-0.886493$

C -4.301570 2.392813-1.255194

$\mathrm{H}-5.3880042 .581975-1.296431$

H -4.095689 $1.609462-2.012923$

C -3.894825 1.8729870 .113481

$\mathrm{H}-4.4561850 .9580480 .366016$

$\mathrm{H}-4.1599822 .6242710 .884807$

C -2.398717 1.5967800 .171799

$\mathrm{H}-2.1497660 .798259-0.548715$

$\mathrm{H}-2.1251821 .1942821 .164118$

C - $0.006328-1.080311-2.598967$

H $0.483852-0.743846-3.536154$

C $0.055061-2.606949-2.552498$

$\mathrm{H}-0.394862-2.939539-1.592682$

H $1.099216-2.967441-2.538347$

C -0.699084 -3.239381-3.715243

$\mathrm{H}-0.198969-2.961827-4.664638$

H $-0.646719-4.340332-3.651610$

C -2.145429 -2.770957 -3.757907

$\mathrm{H}-2.666988-3.131046-2.847430$

$\mathrm{H}-2.678588-3.219500-4.614011$

C - $2.219737-1.252244-3.807410$

H - $1.785465-0.898771-4.763698$

H -3.270261 -0.909323 -3.801378

C - $1.458786-0.624060-2.648156$

$\mathrm{H}-1.5091390 .477445-2.701860$

$\mathrm{H}-1.948809-0.911300-1.694064$

C $2.657819-0.954833-1.492148$

H $2.558226-2.036001-1.253539$

C $3.657538-0.384143-0.492313$

H $3.7544820 .708066-0.668678$

H $3.268209-0.5096290 .536273$

C $5.021002-1.050527-0.618575$

H $4.919877-2.116530-0.332151$

H $5.733682-0.6043910 .097207$

C $5.557086-0.961740-2.039261$

H $6.530408-1.475869-2.122879$

H $5.7458630 .100879-2.292384$

C 4.556782 -1.534742 -3.031641

H 4.940852 -1.459313 -4.064034

H $4.421304-2.616381-2.827175$

C $3.205143-0.839950-2.913880$

H $2.501794-1.264698-3.651550$

H $3.3220340 .229124-3.177102$

C - $1.515907-2.1601432 .912851$

C - $0.125087-2.5484262 .635780$

H $0.546474-2.3494153 .481331$

C $0.318395-3.2823261 .525275$

H -0.423294 -3.760975 0.871462

C $1.688479-3.7766831 .322080$

C $1.966927-4.5532560 .185503$

H $1.148702-4.796147-0.502323$

C $3.256558-5.000967-0.083763$

H $3.447655-5.601975-0.977964$

C $4.299345-4.6919450 .787704$

H $5.313957-5.0454320 .582736$ 
C $4.036436-3.9307751 .928292$

H $4.847628-3.6873072 .620956$

C $2.750260-3.4767072 .192513$

H $2.564255-2.8744213 .087070$

C $-2.514158-2.2835181 .814450$

$\mathrm{H}-2.223000-2.7794330 .882323$

C $-3.730664-1.7254211 .943209$

$\mathrm{H}-3.951140-1.2665172 .917042$

C -4.762495-1.623664 0.917253

C - $4.519239-1.924325-0.434478$

$\mathrm{H}-3.530733-2.285504-0.738904$

C $-5.508082-1.744131-1.393350$

$\mathrm{H}-5.296605-1.978778-2.441631$

C $-6.765628-1.260407-1.026232$

H -7.540739 -1.114072 -1.783883

C -7.025906 -0.9655260 .310368$

$\mathrm{H}-8.008901-0.5885910 .607341$

C $-6.033289-1.1431571 .269320$

H -6.232566 -0.898984 2.318151

\section{L12 $\cdot$ Pd}

$E=-3999.88701681$

Pd $-0.0000800 .000024-0.098598$

P $4.237951-0.122221-0.017110$

P 1.6911691 .6704140 .100346

P -4.238090 $0.122173-0.017114$

P - $1.691257-1.6702810 .101691$

C 3.4604271 .4024660 .177781

C 4.3723332 .5708980 .522176

H 3.9311253 .2257111 .296670

H $4.6124423 .234515-0.335335$

H 5.3481672 .2658200 .939753

C $5.8669830 .148088-0.888535$

H $6.4229070 .762624-0.151629$

C $5.7636641 .001033-2.155021$

H $5.2523670 .429031-2.950479$

H $5.1403471 .890242-1.969726$

C $7.1501431 .397033-2.645206$

H $7.0701092 .011249-3.558380$

H $7.6347102 .039409-1.882988$

C $8.0167660 .171769-2.896678$

H $9.0256450 .466301-3.233007$

H $7.575293-0.417524-3.724854$

C $8.100857-0.704929-1.656183$

H $8.630118-0.153432-0.853643$

H $8.702074-1.608580-1.855980$

C $6.715746-1.096119-1.153049$

H $6.218570-1.722887$-1.919546

H $6.807616-1.723359-0.249244$

C $4.776431-0.9824341 .559633$

H $5.317654-1.9014291 .253691$

C $3.571112-1.3961902 .404188$

H $2.992202-0.4908992 .661425$

H $2.878889-2.0326941 .828199$

C $3.997138-2.1136103 .678217$

H $4.501995-3.0643793 .410102$ 
H 3.107002 -2.394349 4.267669

C $4.942411-1.2562494 .503267$

H $5.255513-1.7840915 .420342$

H $4.404206-0.3458104 .835699$

C $6.150421-0.8473773 .676708$

H $6.830806-0.2061454 .263237$

H 6.731707 -1.752552 3.410342

C $5.731918-0.1308932 .399122$

H 6.6315910 .1465481 .820991

H 5.2229540 .8164502 .665311

C $3.218780-1.387312-0.899867$

H $2.210459-1.250487-0.436962$

C $3.651530-2.847096-0.719773$

H $4.649804-3.000130-1.174558$

H $3.751869-3.1204000 .343586$

C $2.654684-3.792317-1.380699$

H $2.993900-4.836159-1.263099$

H $1.690681-3.718822-0.841354$

C $2.429136-3.459560-2.846419$

H $3.364797-3.643833-3.411917$

H $1.670569-4.135944-3.280447$

C $2.022916-2.003730-3.015485$

H $1.048199-1.819493-2.518558$

H $1.883326-1.756243-4.082473$

C $3.057936-1.081371-2.390211$

H $2.781656-0.026381-2.537577$

H $4.027736-1.236505-2.904142$

C $1.3875313 .011192-1.200908$

H $0.2790633 .056228-1.245282$

C $1.8617042 .536824-2.570980$

H $1.4408591 .535261-2.780664$

H $2.9638082 .413267-2.542062$

C $1.4853833 .505950-3.684286$

H $0.3831813 .512212-3.796896$

H $1.8884333 .154269-4.650354$

C $1.9605974 .919527-3.384942$

H $3.0690294 .936612-3.375505$

H $1.6481605 .614558-4.183962$

C $1.4510515 .384905-2.029408$

H $1.7970566 .410718-1.812001$

H $0.3425655 .430381-2.052005$

C $1.8872714 .428848-0.925500$

H 1.5294554 .7954200 .052553

H $2.9918454 .430591-0.869991$

C 1.2739292 .6177081 .677001

H 1.9037853 .5295741 .732460

C 1.5825601 .7617882 .901276

H 1.0593230 .7884252 .787196

H 2.6630061 .5338652 .928963

C 1.1444772 .4155644 .203376

H 1.7319363 .3407824 .369522

H 1.3676661 .7498085 .056423

C -0.3339872 .7672344 .163016$

$\mathrm{H}-0.9223101 .8324254 .048779$

H -0.660120 3.228296 5.112412

C - 0.6269933 .6838242 .985613 
$\mathrm{H}-0.0903644 .6428233 .131527$

$\mathrm{H}-1.7029643 .9384372 .943742$

C - 0.1912073 .0493631 .672173

$\mathrm{H}-0.3865783 .7472010 .837682$

H -0.805952 2.145331 1.471828

C - $3.460513-1.4023280 .178976$

C $-4.372333-2.5705420 .524348$

$\mathrm{H}-3.931431-3.2243421 .299885$

$\mathrm{H}-4.611857-3.235226-0.332490$

H -5.348454 -2.265136 0.940993

C -5.867144 -0.148840 -0.888276

$\mathrm{H}-6.423121-0.762660-0.150814$

C -5.763863 -1.002973 -2.153962

H -5.140658 -1.892079-1.967793

H -5.252447 -0.431784 -2.949928

C -7.150358 -1.399270 -2.643857

$\mathrm{H}-7.070343-2.014324-3.556468$

H -7.635037 -2.040897 -1.881079

C $-8.016842-0.174143-2.896482$

H -9.025732 $-0.468874-3.232605$

$\mathrm{H}-7.5752630 .414367-3.725161$

C -8.100916 $0.703670-1.656776$

$\mathrm{H}-8.6302610 .152936-0.853769$

$\mathrm{H}-8.7020481 .607194-1.857409$

C -6.715796 $1.095193-1.153930$

$\mathrm{H}-6.8076481 .723227-0.250673$

H -6.218529 $1.721248-1.920952$

C - 4.7765000 .9836851 .558948

$\mathrm{H}-5.3179471 .9023081 .252279$

C -5.7316900 .1326762 .399312$

$\mathrm{H}-6.631432-0.1453491 .821567$

H -5.222551 -0.814386 2.666166

C -6.150056 0.8501053 .676407

$\mathrm{H}-6.7315301 .7549813 .409440$

$\mathrm{H}-6.8302300 .2092284 .263571$

C -4.9419501.259822 4.502403

H -4.403511 0.3497364 .835426

H -5.254957 1.7883125 .419137

C -3.997012 2.116717 3.676487

$\mathrm{H}-4.5021133 .0671803 .407748$

H -3.106797 2.398084 4.265520

C -3.571109 1.398388 2.402929

$\mathrm{H}-2.9919130 .4934412 .660746$

H -2.879179 2.034605 1.826265

C -3.218929 1.386541-0.900921

$\mathrm{H}-2.2106411 .250139-0.437820$

C -3.057926 1.079344 -2.390991

$\mathrm{H}-4.0276701 .234061-2.905153$

$\mathrm{H}-2.7816270 .024231-2.537456$

C -2.022819 2.001175 -3.016903

$\mathrm{H}-1.0481721 .817342-2.519684$

$\mathrm{H}-1.8830841 .752796-4.083666$

C -2.429047 3.457152 -2.849124

$\mathrm{H}-3.3646153 .640965-3.414923$

$\mathrm{H}-1.6703954 .133152-3.283605$

C $-2.6548193 .791158-1.383720$ 
H -2.994082 $4.835093-1.267067$ $\mathrm{H}-1.6908883 .718164-0.844181$ C -3.651728 2.846466 - 0.722122 $\mathrm{H}-3.7522273 .1206730 .340989$ H -4.649941 2.999089-1.177185 C - $1.387621-3.012120-1.198477$ H $-0.279154-3.057139-1.242845$ C -1.861848 -2.538950 -2.568946 $\mathrm{H}-2.963952-2.415359-2.540134$ $\mathrm{H}-1.440995-1.537568-2.779489$ C -1.485527 -3.509041-3.681416 $\mathrm{H}-0.383328-3.515354-3.794042$ $\mathrm{H}-1.888610-3.158210-4.647777$ C -1.960678 -4.922380 -3.380836 $\mathrm{H}-3.069108-4.939504-3.371334$ $\mathrm{H}-1.648248-5.618076-4.179281$ C -1.451061 -5.386566 -2.024921 $\mathrm{H}-0.342574-5.432021-2.047538$ $\mathrm{H}-1.797012-6.412208-1.806611$ C $-1.887269-4.429569-0.921826$ $\mathrm{H}-1.529385-4.7952700 .056524$ H -2.991840 -4.431345 -0.866264 C -1.273936 -2.616268 1.679094 $\mathrm{H}-1.903922-3.5279881 .735443$ C $0.191149-3.0480971 .674397$ H $0.805944-2.1443101 .473123$ H $0.386300-3.7467120 .840504$ C $0.627116-3.6813952 .988338$ H $0.090424-4.6402123 .135221$ H $1.703055-3.9361512 .946492$ C $0.334403-2.7637064 .164963$ H $0.922777-1.8290464 .049754$ H $0.660673-3.2239215 .114722$ C -1.144021 -2.411864 4.205259 $\mathrm{H}-1.731540-3.3368784 .372325$ $\mathrm{H}-1.367011-1.7453245 .057744$ C - $1.582242-1.7592052 .902646$ $\mathrm{H}-1.058872-0.7860292 .787607$ H -2.662651 -1.531096 2.930305

\section{L1·Pd}

$E=-2063.30349062$

$\mathrm{Pd}-1.624480-0.0593292 .059371$

P $1.5682230 .252009-0.260861$

P $-1.507830-0.161495-0.234804$

C $0.0592600 .061292-1.074188$

C $0.0125050 .298744-2.572854$

H $-0.580116-0.475943-3.100248$

H $1.0070450 .282484-3.052530$

$\mathrm{H}-0.4348491 .271571-2.865834$

C $2.942221-0.357173-1.356587$

H $2.9138370 .342931-2.216280$

C $4.343863-0.258168-0.752592$

H $4.427160-0.9646300 .096562$

H $4.5364100 .747688-0.340700$

C $5.402644-0.605735-1.792650$ 
H $5.3748230 .153831-2.599082$ H $6.409531-0.542377-1.345574$ C $5.164375-1.983666-2.391980$ H $5.291881-2.748140-1.599996$ H $5.922773-2.208812-3.161149$ C $3.760051-2.097201-2.966961$ H $3.584929-3.108808-3.371050$ H $3.656263-1.401213-3.823424$ C $2.705579-1.758921-1.921809$ H $1.690357-1.841534-2.341602$ H $2.757800-2.499322-1.102236$ C $1.601015-0.5896761 .378113$ H $0.611646-0.2541591 .784414$ C $2.672950-0.1718472 .385918$ H 2.7866170 .9223842 .446868 H $3.660123-0.5706912 .079442$ C $2.314098-0.7238823 .762004$ H $3.074535-0.4241674 .503680$ H $1.360839-0.2593364 .086935$ C $2.156089-2.2373713 .737689$ H $3.141254-2.6954453 .516900$ H $1.860793-2.6120104 .732833$ C $1.158193-2.6770062 .676573$ H $0.142103-2.3128642 .936833$ H $1.094602-3.7779762 .634272$ C $1.525192-2.1137741 .310836$ H $2.508296-2.5231141 .001435$ H $0.793693-2.4284750 .550115$ C 2.0879352 .0078060 .127869 H 3.0930041 .9672950 .595737 C $2.1886412 .847041-1.147590$ H $2.9393802 .423311-1.838850$ H $1.2178242 .804634-1.679480$ C $2.5360374 .299318-0.846277$ H $2.5793514 .879413-1.784109$ H $3.5530204 .344278-0.408112$ C 1.5477224 .9170530 .129940 H 1.8152245 .9659560 .344193 H $0.5418344 .940675-0.335235$ C 1.4827214 .1003621 .410461 H 0.7566784 .5362422 .117791 H 2.4684864 .1369461 .916746 C 1.1167022 .6520651 .118748 H 0.0990722 .6077800 .692162 H 1.0619202 .0753572 .058701 C -2.635262 $1.111973-1.047677$ $\mathrm{H}-2.5611640 .934302-2.141821$ C $-4.1040070 .965856-0.654960$ $\mathrm{H}-4.1844971 .0520460 .448694$ $\mathrm{H}-4.482886-0.036927-0.916241$ C -4.977970 2.022617-1.321214 $\mathrm{H}-4.9696751 .859738-2.417557$ $\mathrm{H}-6.0278211 .902210-1.001450$ C -4.482837 3.429739-1.027398 $\mathrm{H}-4.5708453 .6256800 .059657$ H -5.115698 4.181386-1.530545 
C -3.027389 3.579394 -1.441226

$\mathrm{H}-2.9473983 .463239-2.540919$

H -2.655122 4.593517 -1.210769

C -2.156556 2.530964 -0.763436

$\mathrm{H}-1.1036842 .643776-1.075162$

H -2.175961 2.6891830 .335233

C - $2.288593-1.740353-0.939526$

C $-1.286170-2.816475-1.341647$

C -3.298785 -2.350050 0.034342

$\mathrm{H}-2.832658-1.431476-1.858759$

C -1.986055 -4.013140 -1.974999

H $-0.751403-3.158258-0.432672$

$\mathrm{H}-0.514231-2.408787-2.014854$

C -4.018110 -3.543536 -0.580400

$\mathrm{H}-2.745290-2.6687800 .942057$

$\mathrm{H}-4.022668-1.5982570 .389961$

C -3.029958 $-4.605346-1.039387$

$\mathrm{H}-1.247092-4.780033-2.267398$

$\mathrm{H}-2.479261-3.687749-2.912703$

$\mathrm{H}-4.740700-3.9689200 .137853$

$\mathrm{H}-4.614389-3.198501-1.449278$

H -3.554066 -5.449182 -1.521268

H -2.518056 -5.025934 -0.151045

1

$E=-2755.07358684$

Pd $1.735680-0.3432010 .718549$

P -2.134037 -0.136494 -0.130948

P $0.6699840 .602403-1.169587$

C - $1.1034950 .494844-1.363283$

C $-1.6712530 .742763-2.748023$

$\mathrm{H}-1.3017761 .694689-3.179739$

$\mathrm{H}-2.7724230 .820363-2.769636$

$\mathrm{H}-1.418684-0.042549-3.491197$

C $-3.8423950 .554060-0.369721$

$\mathrm{H}-4.1374740 .141928-1.356667$

C -4.891860 0.081996 0.637248

$\mathrm{H}-4.6569540 .5056341 .633413$

$\mathrm{H}-4.878169-1.0154540 .753561$

C -6.281942 0.5446070 .216197

$\mathrm{H}-6.5468610 .054467-0.741698$

$\mathrm{H}-7.0331430 .2084250 .951270$

C -6.3384312.0549940.040865

H -6.1757392.537998 1.024766

$\mathrm{H}-7.3419142 .369505-0.293169$

C $-5.2693062 .537883-0.928630$

$\mathrm{H}-5.2951463 .637010-1.020894$

H -5.481960 2.138732 -1.940741

C -3.883583 2.078591-0.495674

H -3.109519 2.434484 -1.194147

$\mathrm{H}-3.6423152 .5323710 .483153$

C - 1.5048210 .2035131 .572497

$\mathrm{H}-0.442394-0.1306111 .471000$

C -2.112732 -0.5897082 .731503$

H $-2.185429-1.6661502 .506957$

$\mathrm{H}-3.145574-0.2400992 .926664$ 
C -1.265636 -0.392225 3.985017

$\mathrm{H}-1.704371-0.9524604 .828675$

H -0.269202 -0.8404453 .802918$

C - 1.1124391 .0788414 .345011

$\mathrm{H}-2.1003061 .4794994 .649693$

$\mathrm{H}-0.4534081 .1934765 .223584$

C -0.592382 1.8944123 .170022

H 0.4444891 .5869102 .923411

$\mathrm{H}-0.5474142 .9660423 .430414$

C -1.459957 1.6854321 .937565

$\mathrm{H}-2.4878902 .0409672 .153669$

$\mathrm{H}-1.0904812 .2805691 .088175$

C -2.411750 -1.986262 -0.131743

$\mathrm{H}-3.154171-2.2160540 .659976$

C -2.980175 -2.455921 -1.471994

H -3.954775 -1.976129-1.675880

$\mathrm{H}-2.298115-2.129807-2.281852$

C $-3.135636-3.970829-1.522131$

H -3.526198 $-4.277431-2.507753$

H -3.896830 -4.280652 -0.778576

C $-1.824875-4.677341-1.212859$

$\mathrm{H}-1.955759-5.772499-1.248027$

$\mathrm{H}-1.081882-4.427704-1.996562$

C -1.289047 -4.236660 0.139749

$\mathrm{H}-0.328110-4.7294560 .367632$

$\mathrm{H}-1.995922-4.5553520 .932317$

C -1.113880 -2.726425 0.197172

H - $0.334682-2.419425-0.524475$

$\mathrm{H}-0.723751-2.4319801 .186202$

C $1.352858-0.200103-2.732059$

H $0.8605330 .312609-3.585905$

C $2.861718-0.025440-2.896396$

H $3.367743-0.464389-2.011091$

H $3.1359611 .043588-2.910748$

C $3.373419-0.697282-4.165504$

H $2.936008-0.186941-5.046725$

H $4.466935-0.569175-4.248655$

C $2.998255-2.170159-4.210548$

H $3.504408-2.698708-3.378030$

H $3.361352-2.639324-5.141454$

C $1.494494-2.343797-4.064700$

H $0.988313-1.886682-4.938692$

H $1.219749-3.413516-4.074258$

C $0.989770-1.679598-2.791538$

$\mathrm{H}-0.102616-1.805534-2.694414$

H $1.439998-2.184316-1.910564$

C $1.1886732 .392932-1.511822$

H $1.3734032 .460369-2.605938$

C $2.4916842 .727332-0.781376$

H 2.299409 2.6671640.311097

H $3.2685651 .967559-0.974012$

C $2.9941634 .118759-1.142103$

H $3.2494814 .142486-2.220978$

H $3.9298314 .342351-0.600183$

C $1.9401435 .177187-0.852360$

H $2.2948686 .178535-1.153011$ 
H 1.7717185 .2245380 .242001

C $0.6237604 .842514-1.539062$

H - $0.1458445 .594321-1.289995$

H $0.7615754 .895141-2.637464$

C $0.1421463 .445602-1.164552$

$\mathrm{H}-0.8250123 .218334-1.643307$

$\mathrm{H}-0.0421643 .417895-0.072384$

Cl $1.766330-2.6347142 .857573$

C $2.796931-1.2796552 .362274$

C $3.694133-1.4478421 .277529$

C $2.944966-0.1816933 .244103$

C $4.752065-0.5286241 .125482$

H $3.662255-2.3642010 .679952$

C 3.9951440 .7077963 .058979

H $2.256004-0.0783994 .088000$

C 4.9121150 .5256812 .013138

H $5.462454-0.6749880 .306009$

H 4.1086721 .5496003 .748061

H 5.7475011 .2205481 .892656

Chlorobenzene

$E=-691.737946394$

C $-0.5098480 .000009-0.000001$

C $0.175213-1.213394-0.000004$

C $1.567783-1.2053070 .000003$

C $2.267271-0.0000020 .000002$

C 1.5677981 .2052980 .000001

C 0.1752161 .2133960 .000004

H - $0.387043-2.150669-0.000007$

H $2.110020-2.1552920 .000002$

H $3.360644-0.0000180 .000003$

H 2.110022 2.1552910.000003

H -0.3870112.1506880.000006

Cl $-2.2510140 .000000-0.000002$

$\mathrm{dba}$

$E=-731.149587103$

O - $0.000024-2.1206150 .000022$

C $0.000002-0.9003860 .000072$

C $1.253926-0.1133250 .000052$

H 1.1719380 .9802150 .000060

C $2.446397-0.7360660 .000024$

H $2.404710-1.8343450 .000016$

C $3.774331-0.1368670 .000003$

C $4.896113-0.980626-0.000046$

H $4.740811-2.064527-0.000066$

C $6.185844-0.459772-0.000068$

H $7.046654-1.134340-0.000106$

C $6.3779000 .919593-0.000041$

H $7.3902521 .333632-0.000058$

C 5.2729161 .7734490 .000009

H 5.4201372 .8572660 .000032

C 3.9863921 .2525250 .000031

H 3.1289501 .9321750 .000072

C -1.253911 -0.113309 0.000050

$\mathrm{H}-1.1720670 .9802360 .000058$ 
$\begin{array}{lll}\text { C }-2.446390 & -0.736067 & 0.000022 \\ \text { H }-2.404745 & -1.834342 & 0.000017 \\ \text { C }-3.774308 & -0.136844 & 0.000001 \\ \text { C }-3.986396 & 1.252538 & 0.000005 \\ \text { H }-3.128994 & 1.932243 & 0.000025 \\ \text { C }-5.272932 & 1.773440 & -0.000015 \\ \text { H }-5.420153 & 2.857258 & -0.000011 \\ \text { C }-6.377908 & 0.919576 & -0.000041 \\ \text { H }-7.390262 & 1.333609 & -0.000057 \\ \text { C }-6.185828 & -0.459787 & -0.000046 \\ \text { H }-7.046615 & -1.134384 & -0.000065 \\ \text { C }-4.896087 & -0.980614 & -0.000025 \\ \text { H }-4.740785 & -2.064516 & -0.000029\end{array}$

PBE0 / def2tzvp + LANL2TZ(f) with PCM (THF)

\section{L1}

$E=-1935.73583553$

P $1.2364430 .260200-0.125805$

$\mathrm{P}-1.706516-0.2038720 .483148$

C -0.361911 -0.047097-0.690154

C - $0.582495-0.036774-2.194993$

$\mathrm{H}-1.4652920 .565986-2.473670$

$\mathrm{H}-0.747643-1.040375-2.630834$

H $0.2577960 .401199-2.759684$

C $2.467340-0.606834-1.229444$

H $2.339613-0.067180-2.186452$

C $3.941064-0.472548-0.831347$

H $4.113169-1.0046550 .120660$

H $4.2127680 .579891-0.654213$

C $4.854635-1.065800-1.904062$

H $4.767988-0.459721-2.824397$

H $5.906754-0.990436-1.583425$

C $4.494863-2.513687-2.219778$

H 4.704727 -3.140465 -1.333719

H $5.136304-2.899662-3.028863$

C $3.021886-2.651704-2.591187$

H $2.767450-3.709720-2.766923$

H $2.834155-2.126511-3.545664$

C $2.112144-2.071623-1.510650$

H $1.055229-2.153942-1.801336$

H $2.225381-2.665868-0.589808$

C $1.456376-0.2183131 .653229$

H 0.5039500 .1501902 .083119

C 2.6248510 .4254502 .413150

H 2.6428171 .5167792 .290047

H 3.5827710 .0510512 .012518

C 2.5412130 .0942363 .903874

H 3.3952070 .5481344 .433215

H 1.6324670 .5640484 .321554

C $2.493492-1.4079314 .159762$

H $3.461565-1.8557223 .868886$

H $2.371842-1.6094745 .236648$

C $1.377384-2.0691353 .359114$

H $0.396312-1.7249223 .732799$

H $1.397870-3.1624263 .499419$ 
C $1.483995-1.7347711 .874129$

H $2.432933-2.1442641 .484554$

H $0.672749-2.2146471 .313248$

C $1.8413212 .040898-0.185823$

H 2.8813582 .0334880 .189653

C $1.8522562 .613299-1.608622$

H $2.4923792 .010698-2.272666$

H $0.8297192 .559794-2.022108$

C $2.3291164 .064661-1.632431$

H $2.3012714 .449148-2.665356$

H $3.3879164 .102515-1.316796$

C $1.4980834 .948517-0.710391$

H $1.8871595 .979927-0.711440$

H $0.4644015 .005590-1.097066$

C 1.4713534 .3868170 .706041

H 0.8210635 .0000561 .351347

H 2.4856404 .4481081 .142167

C 0.9960592 .9358190 .726537

H -0.049130 2.883940 0.387308

H 0.9915202 .5609671 .761495

C -3.059714 $0.960197-0.139412$

$\mathrm{H}-3.3352730 .671456-1.171254$

C -4.317991 0.8572470 .730619

$\mathrm{H}-4.0410621 .0688931 .780352$

$\mathrm{H}-4.716535-0.1695110 .720882$

C -5.412346 1.826947 0.286695

H -5.771625 $1.531878-0.716375$

$\mathrm{H}-6.2820791 .7482270 .960488$

C -4.908790 3.264615 0.232190

$\mathrm{H}-4.6611243 .6013271 .255719$

H -5.701404 $3.939076-0.132536$

C -3.665666 $3.373254-0.643633$

H -3.938569 3.146229-1.690679

$\mathrm{H}-3.2818324 .407615-0.642311$

C -2.573905 2.407379-0.189148

$\mathrm{H}-1.6999642 .479447-0.855752$

H -2.224922 2.7019670 .818222

C -2.594029-1.853802 0.167453

H -3.420225 -1.821712 0.904783

C -1.709231 -3.0358860 .562861$

H -0.832622 -3.064155 -0.110639

$\mathrm{H}-1.318443-2.8809731 .581812$

C $-2.449518-4.3706020 .491197$

$\mathrm{H}-3.242462-4.3834941 .261445$

$\mathrm{H}-1.764467-5.1985140 .740942$

C $-3.082446-4.596805-0.877082$

$\mathrm{H}-3.652185-5.541035-0.888248$

H - $2.283576-4.707903-1.633475$

C -3.976383 -3.424612 -1.265565

H -4.398685 -3.579013 -2.272947

H -4.837416 -3.379809-0.573217

C -3.216948 -2.099185-1.210102

$\mathrm{H}-3.893533-1.275747-1.488181$

H -2.419420 -2.114045 -1.971547

\section{$L 1 \cdot P d(d b a)$}


$E=-2793.36986041$

Pd -0.347252 -1.112742 0.815601

P $1.6717811 .981215-0.096452$

P $0.787621-0.755853-1.211702$

O -2.361347 0.0929903 .603542

C $1.6331130 .803050-1.365425$

C $2.1965511 .218153-2.718843$

H $1.5668790 .849449-3.545852$

H $3.2179730 .843786-2.922223$

H 2.237811 2.310301-2.860872

C $3.2190362 .997961-0.306421$

H $3.0510523 .465815-1.293992$

C 3.4195274 .1380470 .698019

H 3.6122573 .7157191 .699255

H 2.5132884 .7565630 .788341

C 4.6032435 .0159390 .291379

H $4.3617655 .526335-0.658864$

H 4.7515795 .8118901 .039354

C 5.8816734 .2032150 .116402

H 6.1931923 .8028991 .098459

H $6.7038704 .852367-0.226244$

C $5.6758223 .042983-0.852067$

H $6.5918912 .434130-0.922472$

H $5.4897213 .439477-1.867026$

C $4.5001922 .163557-0.431855$

H $4.3525491 .338902-1.143124$

H 4.7335991 .6964780 .537948

C 1.5741301 .2642181 .611982

H 0.7272370 .5342771 .509869

C 1.1898322 .2229752 .748011

H 0.2993342 .8153222 .498553

H 2.0107932 .9423562 .912955

C 0.9230551 .4467154 .038775

H 0.6912252 .1582734 .848021

H 0.0147300 .8321983 .908013

C 2.1007250 .5632354 .435406

H 2.9575851 .1998694 .725000

H $1.844522-0.0321115 .326904$

C $2.526751-0.3474813 .289501$

H $1.725755-1.0788953 .074610$

H $3.420161-0.9306663 .567291$

C 2.8048490 .4505202 .019078

H 3.6442851 .1418922 .210904

H $3.113351-0.2137621 .202043$

C $0.3092423 .266873-0.114422$

H 0.5362023 .9792400 .699669

C $0.2785854 .042265-1.437313$

H $1.2379864 .554647-1.615866$

H $0.1450773 .324409-2.265704$

C $-0.8544055 .066424-1.471556$

$\mathrm{H}-0.8595835 .581242-2.446491$

H $-0.6611995 .845462-0.711419$

C -2.206948 4.420976-1.196620

$\mathrm{H}-3.0030715 .183218-1.188806$

$\mathrm{H}-2.4525323 .724751-2.019015$

C -2.187446 3.6543500 .119826 
$\mathrm{H}-3.1504793 .1457680 .286061$

$\mathrm{H}-2.0646704 .3679710 .955512$

C -1.057325 2.628149 0.161930

$\mathrm{H}-1.2389891 .849059-0.594015$

$\mathrm{H}-1.0710222 .1091541 .132355$

C -0.364246 -0.909400 -2.691316

H $0.254644-0.815568-3.603014$

C - $1.061924-2.272637-2.733764$

$\mathrm{H}-1.612467-2.410147-1.784913$

H - $0.326492-3.090575-2.781410$

C -2.026492 -2.388468 -3.913079

$\mathrm{H}-1.450432-2.367209-4.856405$

$\mathrm{H}-2.534930-3.366834-3.886674$

C -3.047006 -1.256407 -3.927269

$\mathrm{H}-3.703873-1.348332-3.042314$

H -3.703326 -1.338048 -4.809454

C $-2.3557200 .101829-3.895095$

$\mathrm{H}-1.7932590 .245004-4.836038$

H -3.101388 $0.913382-3.853459$

C -1.398100 0.214831-2.711699

$\mathrm{H}-0.8874211 .190216-2.724559$

H - $1.9750550 .177848-1.768919$

C $1.968339-2.182697-1.549821$

H $1.309890-3.065931-1.446541$

C $3.026395-2.295762-0.453922$

H $3.669209-1.396866-0.487400$

H $2.533391-2.3023780 .532566$

C $3.888992-3.545091-0.618606$

H $3.258240-4.438915-0.462038$

H $4.664619-3.5741100 .164960$

C $4.527240-3.611709-2.001825$

H $5.103195-4.544833-2.117824$

H $5.253514-2.784640-2.107248$

C $3.475650-3.493602-3.099606$

H $3.953390-3.497551-4.093677$

H $2.817448-4.381195-3.068821$

C $2.624821-2.234434-2.932073$

H $1.868518-2.191832-3.732064$

H $3.266657-1.346903-3.063175$

C -2.461433 -0.657107 2.636967

C - $1.528262-1.7831362 .471726$

H $-0.908904-1.9489583 .358951$

C - $1.565386-2.7410271 .437757$

$\mathrm{H}-2.417911-2.7404370 .750372$

C $-0.822021-4.0145271 .436811$

C -1.103275 -4.965866 0.441694

$\mathrm{H}-1.872418-4.742523-0.303056$

C $-0.421082-6.1782240 .386901$

H -0.660112 -6.899984-0.398311

C $0.559325-6.4729811 .333186$

H $1.095662-7.4241671 .295244$

C $0.845297-5.5422462 .334533$

H $1.608271-5.7650283 .084799$

C $0.165596-4.3304782 .386586$

H $0.408723-3.6138353 .173804$

C $-3.497456-0.4126711 .594938$ 
H -3.550046 -1.085650 0.736989

C -4.342275 0.6279081 .715782

$\mathrm{H}-4.2135731 .2411172 .615507$

C -5.396931 1.036305 0.790437

C $-5.6434320 .385473-0.432620$

H -5.031159-0.470901-0.724189

C $-6.6544970 .819835-1.281074$

$\mathrm{H}-6.8278570 .299750-2.226576$

C -7.447055 $1.916098-0.931688$

$\mathrm{H}-8.2413592 .255153-1.601144$

C -7.217035 2.5738350 .275190

$\mathrm{H}-7.8316233 .4320280 .558070$

C -6.2029312.138178 1.124189

H -6.024373 2.6551742.070996

$\mathrm{L1}_{2} \cdot \mathrm{Pd}$

$E=-3998.26865895$

Pd $-0.0000160 .000021-0.009516$

P $4.263345-0.145797-0.022486$

P 1.6899661 .6368170 .141223

$P-4.2633990 .145695-0.022529$

P -1.689969-1.636632 0.143077

C 3.4637101 .3620690 .204485

C 4.3719612 .5137230 .620802

H 4.0029593 .0394211 .518794

H $4.5038453 .288072-0.157165$

H 5.3894832 .1857370 .884740

C $5.9037680 .158870-0.874803$

H $6.4625070 .714905-0.100429$

C $5.8170921 .093407-2.087193$

H $5.2860170 .591540-2.911242$

H $5.2228201 .982927-1.836023$

C $7.2123321 .491003-2.563941$

H $7.1338922 .145996-3.447109$

H $7.7086982 .090139-1.778487$

C $8.0674600 .268973-2.882752$

H $9.0826740 .574309-3.184820$

H $7.632861-0.260036-3.750621$

C $8.132728-0.687199-1.696622$

H $8.681456-0.203274-0.867948$

H $8.706935-1.590676-1.960245$

C $6.739416-1.082806-1.206520$

H 6.235973 -1.667479 -1.995591

H $6.829800-1.748364-0.333724$

C $4.810542-1.0429091 .537331$

H $5.323279-1.9633451 .202401$

C $3.602025-1.4406592 .389287$

H $3.047274-0.5278642 .658233$

H 2.896062 -2.052252 1.809491

C $4.015461-2.1899013 .654307$

H $4.469109-3.1587503 .374261$

H $3.121695-2.4302844 .252290$

C $5.012240-1.3907724 .484408$

H $5.332864-1.9677315 .367354$

H $4.516672-0.4813004 .869953$

C $6.217411-0.9860323 .645143$ 
H $6.911471-0.3662764 .236626$

H $6.781250-1.8917933 .355621$

C $5.799922-0.2315002 .383940$

H 6.7001360 .0277661 .804395

H 5.3251510 .7216652 .674894

C $3.259822-1.390547-0.954367$

H $2.240125-1.243717-0.526816$

C $3.681523-2.856264-0.769428$

H $4.706517-3.001629-1.151324$

H $3.702674-3.1377290 .292531$

C $2.739883-3.803774-1.511556$

H $3.093580-4.841216-1.392579$

H $1.745193-3.758929-1.035700$

C $2.602877-3.449753-2.986907$

H $3.568745-3.622501-3.496971$

H $1.874586-4.119438-3.473501$

C $2.196552-1.991506-3.160679$

H $1.187036-1.828668-2.743974$

H $2.142292-1.728960-4.230142$

C $3.170702-1.060148-2.448894$

H $2.869782-0.013949-2.584812$

H 4.166565 -1.174350 -2.911610

C $1.3762452 .953640-1.192498$

H $0.2722673 .012490-1.208565$

C $1.8178632 .430541-2.558916$

H $1.3524071 .446700-2.737246$

H $2.9092842 .260261-2.531538$

C $1.4795273 .384315-3.703165$

H $0.3831003 .425578-3.829078$

H $1.8815542 .989905-4.651702$

C $2.0014394 .793197-3.446982$

H $3.1071014 .780765-3.451812$

H $1.6963225 .472699-4.260347$

C $1.5164215 .314703-2.099263$

H $1.9172196 .324485-1.908381$

H $0.4156835 .418424-2.123675$

C $1.9103274 .371655-0.962552$

H $1.5488494 .778143-0.005071$

H $3.0096184 .349081-0.892727$

C 1.3450072 .6493461 .692157

H 1.9958493 .5420031 .663113

C 1.6941051 .8612752 .953483

H 1.1061590 .9242392 .954047

H 2.7539981 .5641152 .921749

C 1.4053832 .6531884 .226084

H 2.0730253 .5337314 .267319

H 1.6437812 .0437505 .114245

C - 0.0441743 .1223224 .275985

H -0.704089 2.2385114.349975

$\mathrm{H}-0.2275243 .7250815 .181254$

C -0.406498 3.9128113.023655

H 0.1687904 .8571603 .013039

$\mathrm{H}-1.4708294 .2030793 .046460$

C -0.107099 3.124784 1.749569

$\mathrm{H}-0.3519273 .7407850 .869367$

$\mathrm{H}-0.7550042 .2308131 .691070$ 
C -3.463708 -1.361895 0.206125

C -4.371986 -2.513175 0.623446

$\mathrm{H}-4.001952-3.0391161 .520846$

H $-4.505301-3.287425-0.154408$

H -5.388995 -2.184777 0.888878

C -5.903875 $-0.160003-0.874332$

H -6.462359 $-0.715476-0.099363$

C $-5.817225-1.095586-2.085921$

H $-5.222729-1.984761-1.834074$

H -5.286419 -0.594331-2.910512

C -7.212468 -1.493884-2.562067

$\mathrm{H}-7.134059-2.149641-3.444670$

$\mathrm{H}-7.708584-2.092416-1.775996$

C -8.067874 -0.272291-2.881810

H -9.083087 - $0.578067-3.183436$

H -7.633513 0.256028 -3.750218

C $-8.1331210 .684919-1.696513$

$\mathrm{H}-8.6816130 .201618-0.867320$

H -8.707547 1.588051-1.960839

C -6.739804 1.081229-1.206992

$\mathrm{H}-6.8301621 .747525-0.334754$

H -6.236605 $1.665311-1.996656$

C -4.8104361.0445541.536326

H -5.3232401 .9645941 .200413$

C -5.7996420 .2341332 .384058$

H -6.699927 -0.025903 1.804965

H -5.324741 -0.718632 2.676107

C -6.216953 0.9902063 .644398

$\mathrm{H}-6.7808481 .8956013 .353845$

$\mathrm{H}-6.9109160 .3711704 .236748$

C -5.011661 1.395989 4.482991

$\mathrm{H}-4.5160340 .4869914 .869576$

H -5.332167 1.974023 5.365277

C -4.014992 2.1941043.651776

H -4.468663 3.1626233 .370630

H -3.1211302.4351894.249334

C -3.601774 1.4433142 .387607

$\mathrm{H}-3.0470280 .5308192 .657574$

H -2.895866 2.0541641 .806958

C -3.259972 $1.389418-0.955874$

$\mathrm{H}-2.2402451 .243081-0.528239$

C $-3.1709471 .057355-2.450042$

$\mathrm{H}-4.1668311 .171109-2.912821$

$\mathrm{H}-2.8700830 .010993-2.584843$

C -2.196794 $1.987891-3.162901$

$\mathrm{H}-1.1872681 .825505-2.746041$

H -2.142572 $1.724146-4.232071$

C -2.603099 3.446337-2.990753

$\mathrm{H}-3.5689713 .618522-3.500997$

$\mathrm{H}-1.8748114 .115477-3.478101$

C -2.740067 3.802013-1.515797

$\mathrm{H}-3.0937674 .839587-1.397980$

$\mathrm{H}-1.7453603 .757711-1.039922$

C -3.681665 2.855337 -0.772552

$\mathrm{H}-3.7027273 .1379930 .289091$

H -4.706692 $3.000271-1.154531$ 
C -1.376350 -2.954942 -1.189178

$\mathrm{H}-0.272377-3.013840-1.205324$

C - $1.818160-2.433381-2.556115$

$\mathrm{H}-2.909569-2.263020-2.528746$

$\mathrm{H}-1.352700-1.449751-2.735617$

C $-1.480077-3.388475-3.699336$

$\mathrm{H}-0.383674-3.429955-3.825411$

$\mathrm{H}-1.882255-2.995120-4.648247$

C -2.002041-4.797031-3.441440

H -3.107702 -4.784531-3.446089

H - $1.697112-5.477481-4.254084$

C - $1.516812-5.317020-2.093215$

$\mathrm{H}-0.416081-5.420807-2.117712$

H -1.917610 -6.326570 -1.901113

C -1.910443 -4.372664 -0.957494

$\mathrm{H}-1.548732-4.7780780 .000355$

H -3.009715 -4.349967 -0.887408

C - $1.344744-2.6473851 .695123$

$\mathrm{H}-1.995433-3.5401851 .667178$

C $0.107442-3.1225511 .752859$

H $0.755207-2.2285421 .693254$

H $0.352222-3.7395030 .873312$

C $0.407135-3.9091263 .027770$

H $-0.168004-4.8535783 .018278$

H $1.471513-4.1992053 .050750$

C $0.044861-3.1173084 .279275$

H $0.704635-2.2333034 .352187$

H $0.228441-3.7190355 .185184$

C $-1.404784-2.6484754 .229056$

$\mathrm{H}-2.072270-3.5290864 .271335$

$\mathrm{H}-1.643169-2.0381145 .116587$

C -1.693793 -1.857990 2.955633

$\mathrm{H}-1.105961-0.9208832 .955099$

H - 2.753733 -1.561012 2.923717

\section{L1·Pd}

$E=-2062.48912237$

Pd -1.603384 -0.044029 2.011495

P $1.5501110 .262231-0.249186$

$\mathrm{P}-1.522695-0.170081-0.221416$

C $0.0396890 .063016-1.059581$

C $-0.0108470 .299674-2.560311$

H - $0.616375-0.466918-3.078773$

H $0.9811710 .267522-3.038174$

H $-0.4459581 .277028-2.845931$

C $2.922088-0.350883-1.355474$

H $2.8886560 .356227-2.204649$

C $4.331184-0.260254-0.758408$

H $4.415240-0.9614780 .089825$

H $4.5310270 .744348-0.354574$

C $5.389993-0.614146-1.802668$

H $5.3796470 .152395-2.599006$

H $6.392897-0.571383-1.346999$

C $5.142308-1.986000-2.420601$

H $5.282552-2.762362-1.646386$

H $5.889185-2.193805-3.204153$ 
C $3.729344-2.093643-2.984634$

H $3.548252-3.107413-3.377533$

H $3.624707-1.406514-3.844404$

C $2.675779-1.748984-1.934469$

H $1.664015-1.814063-2.358494$

H $2.715808-2.496262-1.125971$

C $1.589537-0.5701421 .398450$

H $0.586198-0.2502421 .794746$

C $2.664379-0.1245082 .396262$

H 2.7189730 .9702422 .476485

H $3.660288-0.4621492 .059533$

C $2.378680-0.7151963 .777873$

H $3.166204-0.4071904 .485364$

H $1.433331-0.2859144 .157173$

C $2.260652-2.2348413 .739653$

H $3.246902-2.6677783 .489160$

H $1.998785-2.6235644 .737484$

C $1.238400-2.6857542 .702787$

H $0.226335-2.3560843 .004394$

H $1.206654-3.7862192 .644699$

C $1.539934-2.0979531 .327470$

H $2.510770-2.4891130 .973442$

H $0.779249-2.4136990 .600426$

C 2.0764212 .0262350 .116627

H 3.0754651 .9788870 .587788

C $2.1888662 .853591-1.170081$

H $2.9397392 .420865-1.850505$

H $1.2232642 .813718-1.704323$

C $2.5498534 .310288-0.885137$

H $2.5886834 .875512-1.830913$

H $3.5681544 .353798-0.456938$

C 1.5700944 .9566860 .086381

H 1.8719565 .9943110 .304102

H $0.5731435 .014931-0.386924$

C 1.4674954 .1465731 .372920

H 0.7214344 .5920042 .051282

H 2.4349504 .1887191 .906937

C 1.1020072 .6902891 .095782

H 0.0875092 .6343460 .671815

H 1.0513242 .1315952 .042740

C -2.671376 1.089815-1.030211

$\mathrm{H}-2.6180150 .895382-2.118721$

C $-4.1317140 .937576-0.597316$

$\mathrm{H}-4.1797411 .0201030 .504771$

H -4.509641 -0.064616 -0.850144

C $-5.0369371 .989389-1.238107$

$\mathrm{H}-5.0775051 .817165-2.329553$

H -6.069167 1.867885-0.869239

C -4.540225 3.406199-0.976458

$\mathrm{H}-4.6100333 .6216000 .105461$

H -5.186172 4.142819-1.482585

C -3.091874 3.563108-1.424326

$\mathrm{H}-3.0382203 .460047-2.523921$

$\mathrm{H}-2.7223374 .576115-1.191187$

C - $2.1906112 .516532-0.774303$

$\mathrm{H}-1.1548712 .630063-1.130292$ 
H -2.164135 2.6796690.319328

C -2.294925 -1.762036 -0.917227

C - $1.279714-2.814952-1.358647$

C -3.275895 -2.394379 0.076969

$\mathrm{H}-2.863716-1.450767-1.815389$

C $-1.967159-4.035134-1.969452$

H $-0.695839-3.139032-0.479340$

$\mathrm{H}-0.553266-2.385628-2.063677$

C -3.976134 -3.617812 -0.511404

$\mathrm{H}-2.706952-2.6814570 .980546$

$\mathrm{H}-4.016171-1.6584830 .424804$

C -2.974497 -4.655440 -1.006771

$\mathrm{H}-1.213707-4.782557-2.270637$

H -2.487661 -3.731325 -2.896470

$\mathrm{H}-4.651753-4.0630690 .238088$

H -4.617752 -3.299831 -1.354086

H -3.495827 -5.501957 -1.484136

H -2.432027 -5.074822 -0.139634

1

$E=-2754.02681187$

Pd $1.742601-0.2838570 .765313$

P - $2.118991-0.159449-0.126044$

P $0.7104070 .522915-1.169228$

C -1.057924 $0.385617-1.373164$

C -1.610605 $0.558072-2.778234$

H -1.230799 $1.479145-3.257882$

H - $2.7084700 .639090-2.808551$

H - $1.354982-0.271798-3.465309$

C -3.809086 $0.575298-0.418099$

H -4.102099 0.111763 -1.378240

C - 4.8893360 .1936170 .600306

H -4.6549240.654943 1.575037

H -4.916797 -0.894254 0.767254

C -6.263868 0.6793040 .140144

H -6.547056 $0.137279-0.780643$

H -7.023523 0.4204990 .895869

C -6.273613 2.179364 -0.134098

H -6.120606 2.721806 0.816760

H -7.260212 $2.493229-0.512283$

C -5.173793 2.572811-1.114934

H -5.158356 3.665699 -1.257130

H -5.393104 2.135960 -2.106588

C -3.802617 2.091627-0.645907

H -3.021386 2.367303 -1.368063

H -3.543955 2.605166 0.293671

C - 1.4937600 .2304751 .573207

H - $0.431146-0.1087551 .490765$

C - $2.141538-0.5307802 .737576$

H -2.155300 -1.614243 2.554435

H -3.193949 -0.218038 2.849805

C -1.395354-0.256162 4.043928

H - $1.898416-0.7828864 .871643$

H - $0.383987-0.6913883 .968977$

C -1.2915641.2341044.348067

H -2.300068 1.6345964 .561529 
$\mathrm{H}-0.6972541 .3966015 .262451$

C -0.689230 1.9978453 .174360

H 0.3626161 .6925753 .028270

H -0.676448 3.0803363 .383125

C - 1.4580071 .7289421 .884488

$\mathrm{H}-2.4901112 .1045072 .000195$

$\mathrm{H}-1.0106962 .2773171 .044481$

C -2.472892 -2.000197-0.068328

$\mathrm{H}-3.210757-2.1655510 .738073$

C -3.082134 -2.490565-1.387899

$\mathrm{H}-4.035661-1.978384-1.594580$

$\mathrm{H}-2.399875-2.226240-2.215021$

C -3.313009-4.000585-1.386125

H -3.720022 -4.315220 -2.361240

H -4.085034 -4.247868 -0.634497

C -2.036645 -4.767856-1.063777

$\mathrm{H}-2.236643-5.851503-1.033686$

$\mathrm{H}-1.302037-4.609100-1.874102$

C -1.438812 -4.2954450 .255738$

$\mathrm{H}-0.490503-4.8176980 .462417$

$\mathrm{H}-2.124361-4.5627121 .081209$

C - $1.200013-2.7874290 .262799$

H - $0.425985-2.531467-0.477814$

$\mathrm{H}-0.783710-2.4853401 .235363$

C $1.417766-0.364200-2.677313$

H $0.9336950 .100070-3.557613$

C $2.930798-0.189518-2.830328$

H $3.422533-0.552026-1.908812$

H $3.1917670 .875678-2.922497$

C $3.477166-0.944913-4.041713$

H $3.072398-0.491028-4.965044$

H $4.572175-0.823978-4.094667$

C $3.104231-2.422286-4.009684$

H $3.604335-2.905382-3.150172$

H $3.475246-2.935073-4.912611$

C $1.596387-2.597215-3.872988$

H $1.102333-2.217052-4.786141$

H $1.335390-3.666762-3.803568$

C $1.055722-1.847943-2.657975$

H $-0.037191-1.963981-2.592344$

H $1.471535-2.293900-1.735081$

C $1.2032962 .300888-1.620897$

H $1.3853392 .296083-2.713266$

C $2.5034652 .712444-0.919223$

H 2.3285292 .6956080 .172313

H $3.2990921 .972304-1.092121$

C $2.9669444 .102449-1.350665$

H $3.2387594 .078874-2.422359$

H $3.8855254 .379456-0.806665$

C $1.8826175 .152076-1.131190$

H $2.2125846 .135516-1.505539$

H $1.7144405 .274387-0.045431$

C $0.5715004 .739689-1.792842$

H - $0.2177765 .478646-1.574059$

H $0.7002054 .741798-2.890986$

C $0.1267203 .348884-1.343494$ 
H -0.822574 $3.070516-1.824047$

$\mathrm{H}-0.0775063 .376037-0.258961$

Cl $1.794183-2.4063363 .055857$

C $2.805831-1.1100452 .411332$

C $3.654229-1.3723371 .299797$

C 3.0353500 .0338503 .220410

C $4.756214-0.5168901 .072078$

H $3.587349-2.3290700 .777399$

C 4.1176730 .8607782 .952372

H 2.3882070 .2153864 .080634

C 4.9946760 .5748961 .892903

H $5.436964-0.7473460 .248683$

H 4.2903571 .7337563 .586394

H 5.8591991 .2172891 .712189

Chlorobenzene

$E=-691.515163185$

C 2.2672350 .0000210 .000006

C $1.5668511 .205861-0.000023$

C 0.1729881 .2138990 .000017

C $-0.512896-0.000068-0.000036$

C $0.173015-1.213921-0.000033$

C $1.566954-1.2057970 .000028$

H 3.3594550 .0001030 .000023

H $2.1082952 .154932-0.000007$

H -0.385482 2.1513370.000035

H -0.385275 -2.151466 -0.000024

H 2.108346 -2.154896 0.000050

$\mathrm{Cl}-2.2476600 .0000010 .000010$

dba

$E=-730.835506046$

O $0.000004-2.107405-0.000163$

C - $0.000012-0.886857-0.000078$

C $1.257307-0.101695-0.000038$

H 1.1777920 .9893170 .000039

C $2.449364-0.728703-0.000073$

H $2.402240-1.824482-0.000115$

C $3.781913-0.134545-0.000038$

C $4.900342-0.9853700 .000231$

H $4.741794-2.0671160 .000391$

C $6.194258-0.4721280 .000303$

H $7.049562-1.1520380 .000521$

C 6.3959950 .9068090 .000092

H 7.4100221 .3139830 .000142

C $5.2953551 .767635-0.000196$

H $5.4488392 .849542-0.000379$

C $4.0045471 .254668-0.000261$

H $3.1548271 .940880-0.000509$

C -1.257308 -0.101679-0.000014

$\mathrm{H}-1.1777190 .9893260 .000066$

C -2.449358 $-0.728684-0.000053$

$\mathrm{H}-2.402254-1.824464-0.000134$

C -3.781923 $-0.134540-0.000000$

C -4.004554 1.2546740.000104

H -3.154817 1.9408670 .000149 
$\begin{array}{lll}\text { C }-5.295358 & 1.767636 & 0.000151 \\ \text { H }-5.448853 & 2.849543 & 0.000232 \\ \text { C }-6.395996 & 0.906802 & 0.000095 \\ \text { H }-7.410024 & 1.313971 & 0.000132 \\ \text { C }-6.194254 & -0.472134 & -0.000009 \\ \text { H }-7.049556 & -1.152047 & -0.000053 \\ \text { C }-4.900334 & -0.985376 & -0.000056 \\ \text { H }-4.741785 & -2.067122 & -0.000137\end{array}$

\subsubsection{Energies and Coordinates Related to Scheme 2}

Table S12. SCF energies and enthalpy/free energy corrections for Scheme 2. Relative energies are given relative to arene complex 1.

\begin{tabular}{|c|c|c|c|c|c|c|}
\hline & Corr(H) & Corr(G) & $\mathrm{E}(\mathrm{SCF})$ & $E(P C M)$ & $\Delta \mathbf{G}(\mathbf{P C M}) / \mathbf{E}_{\mathrm{H}}$ & $\begin{array}{c}\Delta \mathbf{G}(\mathrm{PCM}) \\
/ \mathrm{kJ} / \mathrm{mol}\end{array}$ \\
\hline 1 & 0.993434 & 0.867313 & -2754.187606 & -2754.192664 & 0 & 0 \\
\hline TS & & & & & 0.018711 & 49.125 \\
\hline $\begin{array}{c}\text { (oxidative } \\
\text { addition) }\end{array}$ & 0.992928 & 0.868232 & -2754.16983 & -2754.174872 & & \\
\hline cis-2 & 0.995407 & 0.872005 & -2754.212956 & -2754.227583 & -0.030227 & -79.360 \\
\hline $2_{2}$ & 1.994381 & 1.77254 & -5508.493847 & -5508.503439 & -0.040098 & -113.19 \\
\hline cis-trans- & & & & & -0.022229 & -58.362 \\
\hline $\begin{array}{c}\text { isomeriza } \\
\text { tion }\end{array}$ & 0.994954 & 0.873402 & -2754.207516 & -2754.220982 & & \\
\hline trans-2 & 0.996002 & 0.872357 & -2754.208345 & -2754.221645 & -0.023937 & -62.845 \\
\hline 3 & 1.097191 & 0.963834 & -2889.30644 & -2889.314253 & -0.039171 & $-110.75^{[\mathrm{a}]}$ \\
\hline $\mathrm{HNMe}_{2}$ & 0.097941 & 0.067181 & -135.0506482 & -135.0530774 & & \\
\hline
\end{tabular}

[a] Correction of the energy due to the differences in the concentration lowers the energy of 3 by 18.8 $\mathrm{kJ} \cdot \mathrm{mol}^{-1}$

TS (oxidative addition)

$E=-2754.17487242$

Pd $-1.396513-0.202960-1.329754$

P $2.193704-0.0637170 .082781$

P $-0.743256-0.4082870 .866893$

C $0.983907-0.3368711 .280086$

C $1.425343-0.7544052 .672441$

H $0.921222-1.6772813 .009161$

H 1.2246280 .0078273 .448043

H $2.501731-0.9770272 .735591$

C 3.7299080 .5442700 .916987

H $4.034824-0.3180471 .536940$

C $4.8911480 .844538-0.033935$

H $4.6289041 .707553-0.669513$

H $5.075819-0.000594-0.714641$

C 6.1611571 .1654710 .750378

H 6.4803980 .2608711 .299189

H 6.9798371 .4101750 .054445 
C 5.9432152 .3039711 .740403 H 5.7443173 .2360011 .180716 H 6.8601272 .4837722 .324534 C 4.7650852 .0194022 .665415 H 4.5916992 .8724463 .340992 H 5.0053831 .1555963 .311830 C 3.4963801 .7169191 .874643 H 2.6556461 .5010882 .547606 H 3.2103572 .6110311 .300545 C $1.6598231 .101666-1.236895$ H $0.6105590 .745523-1.401285$ C $2.3786041 .036447-2.586667$ H $2.4770280 .003895-2.947053$ H $3.4037471 .434952-2.490609$ C $1.6058181 .857474-3.618085$ H $2.1235751 .821169-4.590284$ H $0.6170311 .388366-3.769075$ C $1.4142093 .302316-3.171777$ H $2.3975973 .806760-3.143025$ H $0.8059853 .851802-3.908437$ C $0.7768173 .377403-1.789654$ H - $0.2536162 .983762-1.835425$ H $0.7016414 .424090-1.452757$ C $1.5587392 .554262-0.773195$ H $2.5729032 .980196-0.672121$ H 1.0848132 .6074230 .214657 C $2.774817-1.575735-0.844701$ H $3.564906-1.259056-1.549211$ C $3.361707-2.6066810 .123891$ H $4.252520-2.2018050 .630219$ H $2.616404-2.8112780 .912784$ C $3.727624-3.911982-0.576128$ H $4.111582-4.6344860 .162371$ H 4.554363 -3.723103 -1.284979 C $2.542927-4.495966-1.334243$ H $2.836380-5.423946-1.851100$ H $1.751785-4.774500-0.614850$ C $1.984441-3.482839-2.324607$ H $1.100445-3.890543-2.841050$ H $2.739045-3.284586-3.107859$ C $1.609996-2.176671-1.634553$ H $0.778009-2.355009-0.937628$ H $1.217787-1.460280-2.372181$ C - $1.399283-1.9946191 .607143$ $\mathrm{H}-1.192424-1.9624812 .692466$ C -2.910399 -2.119203 1.409711 $\mathrm{H}-3.116169-2.0970250 .324827$ H -3.439295 -1.251966 1.834234 C - $3.453548-3.4103042 .014625$ $\mathrm{H}-3.327881-3.3781863 .112489$ H -4.537990 -3.485965 1.830152 C -2.733740 -4.635600 1.463046 $\mathrm{H}-2.959458-4.7307730 .385281$ $\mathrm{H}-3.109713-5.5543731 .942301$ C -1.224998 -4.514654 1.644093 H -0.983511 -4.534464 2.722570 
H -0.711655 -5.381622 1.195497

C -0.695948 -3.2208391.035072

H $0.391312-3.1361561 .185643$

H $-0.860927-3.237780-0.057744$

C - 1.6868800 .8641181 .873073

$\mathrm{H}-2.7065080 .8125381 .447107$

C - 1.1471072 .2607991 .584472

$\mathrm{H}-0.0979392 .3067801 .928944$

$\mathrm{H}-1.1345652 .4269090 .495678$

C -1.963594 3.347221 2.275061

$\mathrm{H}-2.9771553 .3619141 .837724$

$\mathrm{H}-1.5212994 .3375812 .075065$

C -2.070372 3.101889 3.774999

$\mathrm{H}-2.7015193 .8711584 .249736$

$\mathrm{H}-1.0681313 .1953534 .232279$

C -2.619058 1.7091034 .059809

$\mathrm{H}-2.6667431 .5262005 .146157$

$\mathrm{H}-3.6572811 .6453093 .685990$

C - -1.7814140 .6277373 .381394$

$\mathrm{H}-2.212332-0.3614183 .599268$

H -0.7682260 .6293383 .817303$

Cl -2.531466 $0.101527-3.444253$

C -3.275850 $0.758342-1.829683$

C -4.337386 0.021891-1.272909

C -3.195122 2.147426-1.626215

C -5.206860 $0.652393-0.390302$

H -4.443796-1.037740 -1.508658

C -4.083668 2.755309-0.745508

H -2.426587 2.727051-2.139095

C -5.083177 2.015917-0.109865

$\mathrm{H}-6.0007750 .0660320 .079463$

H -3.994033 $3.828819-0.560784$

H -5.776558 2.5029980 .578503

cis-2

$E=-2754.22758296$

Pd -1.006766 0.206601 1.383585

P $2.0675750 .241864-0.298342$

P $-0.870030-0.511514-0.782207$

C $0.816220-0.398012-1.306712$

C $1.154769-0.628189-2.773847$

H $0.241644-0.820117-3.352917$

H $1.805484-1.504026-2.951446$

H $1.6345510 .234774-3.269594$

C $3.661865-0.325563-1.044640$

H $3.509220-0.055088-2.104932$

C $4.9467770 .374954-0.596790$

H 5.1619010 .1285900 .455844

H $4.8435401 .468842-0.648352$

C $6.118964-0.066873-1.471475$

H $5.9484830 .292578-2.502541$

H $7.0456860 .417846-1.124792$

C $6.284604-1.582307-1.488682$

H $6.581174-1.924985-0.480781$

H $7.104940-1.868902-2.166010$

C $4.990135-2.281698-1.888623$ 
H $5.112339-3.375582-1.840134$

H 4.747552 -2.041479 -2.939729

C $3.834532-1.846283-0.994909$

H 2.896374 -2.349881-1.273058

H $4.056672-2.1495900 .041037$

C $1.935773-0.2277141 .482144$

H 0.9932620 .2684281 .826033

C 3.0108620 .3834752 .390175

H 3.1159691 .4645592 .218249

H $3.984262-0.0759602 .145329$

C 2.6733310 .1254453 .857596

H 3.4666880 .5484484 .494648

H 1.7385130 .6578394 .101635

C $2.487890-1.3595464 .146738$

H $3.452160-1.8860684 .016863$

H $2.192123-1.5029285 .197967$

C $1.447554-1.9733233 .217129$

H $0.461382-1.5256003 .430900$

H $1.356544-3.0570143 .394935$

C $1.802842-1.7240211 .755754$

H $2.761572-2.2168011 .528053$

H $1.055022-2.1613951 .086316$

C $2.2337432 .095159-0.244884$

H 3.1893572 .3137370 .263881

C $2.3068262 .646298-1.672663$

H $3.1796732 .231672-2.203058$

H $1.4163552 .305368-2.227204$

C $2.3646464 .170174-1.697883$

H 2.376679 4.523195 -2.741739

H $3.3151214 .506019-1.244765$

C $1.2001484 .785541-0.932935$

H $1.2707705 .884999-0.945547$

H $0.2530744 .528128-1.440401$

C 1.1630824 .2698740 .499211

H 0.3014954 .6859941 .044640

H 2.0655724 .6193501 .033181

C 1.1046902 .7470620 .558009

H 0.1331172 .4017660 .166272

H 1.1276472 .4269591 .610242

C -1.887930 0.441030-2.030499

$\mathrm{H}-1.4627120 .161530-3.012521$

C -3.381657 $0.103915-2.068422$

H $-3.8307850 .284060-1.081025$

H -3.534487 -0.962159-2.292166

C $-4.1134210 .945297-3.111155$

$\mathrm{H}-3.7532640 .675876-4.121491$

H -5.188288 $0.703448-3.088562$

C -3.897468 2.436263 -2.885637

H -4.336309 2.720049 -1.912362

H -4.425077 $3.024747-3.653788$

C -2.411168 2.769779-2.881947

$\mathrm{H}-1.9908502 .570421-3.884905$

$\mathrm{H}-2.2522263 .843371-2.686418$

C -1.661219 1.940012 -1.847180

$\mathrm{H}-0.5839582 .152232-1.896007$

H -1.992664 2.226599-0.834981 
C -1.539045 -2.234719-0.990680

$\mathrm{H}-2.613613-2.099085-0.773077$

C -0.987491-3.210684 0.043848

H $0.089483-3.359091-0.149793$

H -1.070527 -2.780993 1.056179

C - $1.699801-4.557324-0.030263$

$\mathrm{H}-2.760626-4.4190770 .247184$

$\mathrm{H}-1.273697-5.2524450 .711340$

C - $1.617339-5.154055-1.430649$

H -2.179019 -6.100907-1.481392

H - $0.564166-5.405466-1.653009$

C -2.126136 -4.175070 -2.482556

$\mathrm{H}-2.008796-4.600263-3.492793$

H -3.209529 -4.012518 -2.337354

C -1.406872 -2.831167-2.392789

$\mathrm{H}-1.801989-2.144099-3.157856$

$\mathrm{H}-0.337131-2.976152-2.620257$

Cl -0.9469861 .0458203 .574405$

C -2.960852 0.2219701 .310697

C -3.619945 1.4531861 .252875

C -3.702978 -0.9576841 .390424$

C -5.0142531 .4954411 .216046$

H -3.049320 2.383894 1.242985

C -5.098867 -0.9068531.357136

$\mathrm{H}-3.203017-1.9243351 .479602$

C -5.7584770 .3162501 .253979$

H -5.521095 2.462750 1.162557

H -5.671570 -1.836550 1.412723

H -6.849857 0.3523871 .220944

$2_{2}$

$E=-5508.50343871$

Pd $1.4129671 .150995-0.000667$

C 1.5243162 .8937520 .917426

P $3.4975891 .410676-0.971200$

Cl -0.6944210 .8815491 .207359$

Cl $0.694306-0.881317-1.207434$

P $4.672605-1.3199570 .108281$

C $4.7408900 .172570-0.745007$

C $3.269625-1.4265541 .288154$

C $6.290785-1.5799230 .996169$

C $4.565699-2.862382-0.937056$

C $3.2782471 .603256-2.815538$

C $4.2524083 .068842-0.510332$

C $6.0383540 .439135-1.495470$

H $6.5674611 .339883-1.136660$

H $5.8913880 .572204-2.583103$

H $6.757445-0.387480-1.398022$

C $5.709819-2.970600-1.950925$

C $3.224870-2.969374-1.662326$

H $4.653206-3.706048-0.227693$

C $5.609786-4.254902-2.770481$

H 5.663387 -2.103153 -2.631176

H $6.692543-2.931702-1.454757$

C $4.263855-4.372544-3.474911$

H $5.746596-5.121948-2.098729$ 
H 6.435497 -4.294370 -3.499686

C $3.118481-4.250760-2.479353$

H 4.177098 -3.566027 -4.225572

H $4.202436-5.322824-4.029893$

H $3.129408-5.121711-1.798225$

H $2.143474-4.275129-2.991662$

H $2.377616-2.901313-0.969824$

H $3.116993-2.099327-2.325786$

C $6.344348-2.7982141 .921753$

C $6.807156-0.3294741 .710259$

H $6.994158-1.7808670 .168115$

C $7.760637-3.0162872 .448634$

H $5.665847-2.6389082 .776621$

H $5.992885-3.7055241 .406975$

C $8.297697-1.7722993 .146402$

H $7.776509-3.8805253 .132306$

H 8.423372 -3.276332 1.603279

C $8.221308-0.5520302 .236123$

H $7.699783-1.5823594 .056279$

H $9.332956-1.9388673 .485270$

H $8.907483-0.6915211 .380720$

H 8.5690380 .3484222 .767685

H 6.7762070 .5301431 .028740

H $6.140893-0.0749012 .549267$

C $3.441155-0.5081842 .501585$

C $2.874164-2.8412481 .728707$

H $2.430271-1.0302170 .686730$

C $2.177006-0.4851183 .353838$

H $4.282575-0.8664033 .118929$

H 3.6991550 .5064432 .163740

C $1.757511-1.8873543 .772395$

H $1.359548-0.0217412 .775622$

H 2.3388520 .1509624 .239761

C $1.593830-2.7834352 .553623$

H $2.520676-2.3215294 .444679$

H $0.821529-1.8485574 .349625$

H $0.785958-2.3861431 .914373$

H $1.292564-3.8010702 .851747$

H $2.717211-3.4984870 .863940$

H $3.676558-3.2942622 .333668$

C $3.1060630 .278374-3.548290$

C $2.1397052 .562639-3.152191$

H $4.2231292 .050758-3.171376$

C $2.0158322 .776411-4.658364$

H $1.2030422 .133935-2.756483$

H $2.2756333 .529835-2.640920$

C $1.8864891 .455006-5.408927$

H $1.1540673 .429379-4.876104$

H $2.9105063 .314188-5.022066$

C $3.0189670 .498783-5.054215$

H $1.8529611 .633258-6.496251$

H $0.9268990 .977890-5.141593$

H $3.9767460 .913741-5.419472$

H $2.881109-0.466014-5.569603$

H $2.190210-0.220436-3.191495$

H $3.943765-0.391059-3.302686$ 
C 4.9737962 .9630130 .830689

C $5.1160783 .808565-1.536463$

H $3.3649653 .700020-0.346522$

C $5.5006005 .187280-1.000876$

H $6.0306133 .238239-1.768182$

H $4.5761163 .933126-2.487042$

C 6.2074675 .1006030 .346499

H $6.1325685 .712595-1.735981$

H $4.5826935 .792192-0.887843$

C 5.3655574 .3365721 .361110

H 6.4445056 .1101250 .720554

H 7.1754384 .5828990 .213764

H 4.4460364 .9054151 .586730

H 5.9102454 .2323472 .314197

H 5.8733872 .3375990 .708919

H 4.3357172 .4442731 .560094

C 1.8973793 .0375152 .257087

C 1.0047724 .0111030 .246980

C 1.7740914 .2691082 .904649

H 2.2746522 .1806962 .817931

C 1.2655105 .3767162 .228095

H 2.0755514 .3585073 .952085

C 0.8746595 .2399330 .896230

H 0.4639676 .0962750 .353817

H $0.6909073 .926298-0.796810$

Pd -1.412934-1.1509620.000806

$\mathrm{H}-2.3777312 .9015550 .969419$

H -2.430281 $1.030208-0.686867$

$\mathrm{H}-2.1900140 .2204983 .191458$

C -3.2249712.969649 1.661932

C $-3.2696451 .426466-1.288325$

C -3.105910 -0.278190 3.548317

C - 4.5658022 .8624810 .936695

C -3.118646 4.251148 2.478787

H -3.117022 2.099692 2.325498

P -3.497581-1.410593 0.971289

C -1.524098 -2.893838 -0.917068

$\mathrm{P}-4.6726321 .319927-0.108452$

C -3.441148 $0.507985-2.501677$

C $-2.8742372 .841139-1.729003$

C $-3.278269-1.6030922 .815636$

C -3.018781 -0.498523 5.054252

H -3.943544 0.3913273 .302713

H -4.653358 3.7060510 .227223

C -5.709919 2.970772 1.950554

C -4.264020 4.373003 3.474339

$\mathrm{H}-3.1296265 .1220081 .797543$

H -2.143637 4.2756372 .991084

C -4.740893 -0.172522 0.744979

C -4.252455 -3.068763 0.510525

C - $1.897108-3.037782-2.256725$

C $-1.004417-4.011050-0.246501$

C $-6.2908301 .579720-0.996360$

$\mathrm{H}-4.2826080 .866102-3.119027$

H -3.699073 -0.506631 -2.163739

C -2.177024 $0.484917-3.353964$ 
C -1.593935 2.783327 -2.553968

$\mathrm{H}-2.7172913 .498454-0.864294$

$\mathrm{H}-3.6766543 .294079-2.333988$

H -4.223207 -2.050464 3.171494

C -2.139847 -2.562607 3.152329

C $-1.886409-1.4548705 .408969$

$\mathrm{H}-3.976599-0.9133285 .419585$

$\mathrm{H}-2.8807720 .4662905 .569570$

H -5.663428 2.1034142.630916

H -6.692646 2.9317511 .454402

C -5.609951 4.255187 2.769938

H -4.1772093.5665934.225109

$\mathrm{H}-4.2026545 .3233624 .029190$

C -6.038383 -0.4390861.495403

$\mathrm{H}-3.365038-3.6999650 .346681$

C -5.116086 -3.808425 1.536726

C -4.973918 -2.962962 -0.830457

$\mathrm{H}-2.274465-2.181067-2.817669$

C - $1.773645-4.269425-2.904159$

C $-0.874123-5.239929-0.895625$

$\mathrm{H}-0.690567-3.9260930 .797281$

H -6.994196 $1.780769-0.168322$

C -6.344456 2.797874-1.922121

C $-6.8071900 .329155-1.710250$

$\mathrm{H}-1.3595270 .021622-2.775738$

$\mathrm{H}-2.338866-0.151231-4.239838$

C -1.757603 1.887131-3.772648

H -0.785993 2.386159-1.914724

$\mathrm{H}-1.2927723 .800955-2.852214$

$\mathrm{H}-1.203132-2.1340352 .756603$

H -2.275897 -3.529808 2.641097

C $-2.015989-2.7763114 .658512$

$\mathrm{H}-1.852825-1.6330266 .496309$

H -0.926776 -0.977906 5.141511

H -5.746820 5.1221332 .098069

H -6.435656 4.294705 3.499147

H -6.567387 -1.339938 1.136697

H -5.891478 -0.571971 2.583065

H -6.7575430 .3874511 .397777$

H -6.030604 -3.238079 1.768467

H -4.576071 -3.932942 2.487282

C -5.500648 -5.1871591 .001212$

C $-5.365700-4.336541-1.360811$

H $-5.873506-2.337547-0.708664$

$\mathrm{H}-4.335876-2.444245-1.559910$

H $-2.075070-4.358969-3.951592$

C - $1.264931-5.376899-2.227482$

$\mathrm{H}-0.463323-6.096160-0.353118$

H -5.665979 2.638453 -2.776987

H -5.992999 3.705272 -1.407495

C -7.760767 3.015834-2.448989

C -8.221373 $0.551597-2.236082$

$\mathrm{H}-6.776174-0.530363-1.028609$

$\mathrm{H}-6.1409620 .074488-2.549257$

$\mathrm{H}-2.5207852 .321223-4.444970$

H $-0.8216121 .848321-4.349866$ 
H -1.154345 -3.429430 4.876285

$\mathrm{H}-2.910754-3.3138945 .022267$

H -6.132590 -5.712439 1.736365

$\mathrm{H}-4.582752-5.7920830 .888163$

C -6.207573 -5.100529 -0.346134

H -4.446191 -4.905398 -1.586446

H -5.910428 -4.232352 -2.313879

$\mathrm{H}-7.7766823 .879964-3.132798$

H -8.423478 $3.275998-1.603652$

C -8.297828 $1.771726-3.146544$

$\mathrm{H}-8.9075110 .691204-1.380668$

H -8.569108 $-0.348944-2.767490$

H $-6.444633-6.110065-0.720137$

H -7.175534 -4.582813 -0.213378

$\mathrm{H}-7.6999481 .581658-4.056417$

H -9.333104 1.938221 -3.485395

H 1.1686776 .3388422 .736907

H -1.167963 -6.339065 -2.736193

TS cis-trans isomerization

$E=-2754.22098227$

Cl $0.4027862 .004478-3.115468$

Pd $1.3452710 .621355-1.454200$

$\mathrm{H}-1.9890132 .109130-1.716914$

H - $0.9205440 .044383-1.787145$

H 0.7591913 .1605680 .221170

C -2.252184 2.481795 -0.717834

C -1.741369 -0.636966 -1.487054

C 0.7855852 .9743911 .310259

C -3.027840 1.4465350 .099296

C $-3.0108233 .797540-0.840539$

$\mathrm{H}-1.2886012 .662197-0.220584$

P 1.0544360 .2245980 .747187

C $2.837421-0.642915-1.429340$

P - $2.045131-0.1244060 .249676$

C -1.211437 -2.063304-1.633864

C -2.889023 -0.387106 -2.474640

C 1.6822761 .7718011 .583723

C 1.2895784 .2202282 .030903

H -0.2466152 .7454551 .613844$

H -3.956831 $1.184827-0.439062$

C -3.409076 2.020147 1.467307

C -3.388987 4.358456 0.523679

$\mathrm{H}-3.9282883 .637915-1.436315$

H -2.397322 4.517438 -1.404709

C -0.626052 0.0011351 .228876

C $2.101417-1.1144751 .537300$

C $2.651225-1.991338-1.754198$

C $4.142965-0.150386-1.301102$

C -3.219871-1.290967 1.090186

H -2.010502 -2.784764 -1.389542

H $-0.396980-2.236472-0.915398$

C -0.739040 -2.306733 -3.064065

C -2.403997 -0.628630 -3.902111

$\mathrm{H}-3.2693180 .639672-2.393009$

H -3.734629 -1.059290 -2.255343 
H 1.6477041 .5632722 .668009

C 3.1260752 .0885951 .198503

C 2.7384114 .5298061 .672194

H 1.2095394 .0638363 .122419

H 0.6407225 .0788101 .791971

H -2.487306 2.1979832.047026

H -4.012348 1.3006912 .044339

C -4.1741163.333644 1.332385

$\mathrm{H}-2.4685064 .6262061 .073943$

$\mathrm{H}-3.9691125 .2891450 .415897$

C - 0.8550350 .0479902 .735005

H $3.027159-1.1051560 .938530$

C $2.513936-0.9129042 .999411$

C $1.444985-2.4798911 .348570$

H $1.647626-2.396821-1.882016$

C $3.750165-2.834345-1.930486$

C $5.237314-0.998435-1.480469$

H $4.3184690 .900499-1.066158$

H -3.505861 -0.7387052 .003535$

C -4.515037-1.580788 0.328166

C - $2.558449-2.5929391 .548189$

H $0.110717-1.635305-3.276672$

H - $0.363224-3.338260-3.164450$

C -1.845954 -2.035296-4.074426

$\mathrm{H}-1.6195410 .112516-4.133260$

H -3.232090 -0.452257 -4.607341

H 3.1525812 .2718290 .109604

H 3.7881431 .2285181 .385834

C 3.6380203 .3262361 .928575

H 3.0967725 .4073182 .234339

H 2.7945384 .7989650 .602202

H -5.141394 3.139680 0.834180

H -4.414152 3.7276062 .333346

H - $0.440377-0.8277953 .266854$

H -0.412244 0.9429163 .207318

$\mathrm{H}-1.9216020 .0827082 .998991$

H $1.625636-0.8880143 .651574$

H 3.0283700 .0498263 .134167

C $3.439644-2.0415683 .449584$

C $2.361312-3.6078781 .806839$

H $0.498782-2.5057101 .914853$

H $1.172838-2.6238340 .294259$

H $3.584068-3.884474-2.185514$

C $5.046963-2.345392-1.784749$

H $6.248884-0.595862-1.380248$

H -4.281248 -2.153466 -0.584630

H $-5.001531-0.649592-0.000522$

C $-5.474641-2.3967511 .190822$

C -3.517312 -3.411134 2.406912

$\mathrm{H}-1.633097-2.3743762 .097349$

$\mathrm{H}-2.259057-3.1848230 .668671$

H - 2.657301 -2.774257 -3.937469

$\mathrm{H}-1.467228-2.173872-5.099574$

H 4.6724533 .5445601 .617114

H 3.6769223 .1199383 .013941

H $3.722532-1.8950154 .504862$ 
H 4.375752 -1.988414 2.864852

C $2.803759-3.4120703 .251555$

H $3.247963-3.6345761 .148301$

H $1.852959-4.5786951 .686683$

H -6.393285 -2.615711 0.623114

H -5.784053 -1.7887712.060292

C -4.828376 -3.687675 1.679885

$\mathrm{H}-3.726598-2.8579873 .340707$

H -3.036883 -4.355797 2.708419

H 3.504022 -4.207733 3.553631

H 1.924826 -3.502328 3.916004

$\mathrm{H}-4.629081-4.3422390 .812040$

H -5.522652 -4.241520 2.332003

H 5.905249 -3.008289 -1.916546

trans-2

$E=-2754.22164473$

Cl -0.1500432 .2384912 .573365$

Pd -1.4612600 .5718191 .523548$

H 2.374422 2.0571171 .604144

H 0.9749150 .1395971 .779467

H -0.374065 3.2129930 .047754

C 2.7176312 .3418130 .600935

C $1.699659-0.6539431 .512221$

C $-0.4847693 .135448-1.048188$

C $3.2715771 .148869-0.178727$

C 3.7328043 .4743530 .693946

H 1.8127262 .7000110 .091315

P - $1.0236720 .388096-0.686259$

C -2.929612 -0.7250691 .361763$

P $2.047392-0.250556-0.240533$

C $1.002756-2.0016421 .705769$

C $2.871036-0.5139762 .492488$

C -1.508503 $2.054052-1.371710$

C - $0.8971364 .484587-1.627704$

H $0.4967722 .835012-1.444524$

H 4.1498230 .7479550 .360124

C $3.7330271 .600355-1.568223$

C $4.2106283 .914072-0.683626$

H 4.6007903 .1423731 .293103

H 3.2853014 .3200431 .239629

C $0.6297980 .041447-1.179640$

C $-2.175167-0.773079-1.606129$

C $-2.781350-2.0888251 .645467$

C $-4.228426-0.2150411 .200836$

C $3.026595-1.606749-1.053939$

H 1.710892 -2.820998 1.491438

H $0.172372-2.1008520 .990782$

C $0.501146-2.1375843 .139836$

C $2.352769-0.6304573 .923830$

H 3.3810080 .4506562 .370974

H $3.623187-1.2988632 .308559$

$\mathrm{H}-1.5216781 .918547-2.468488$

C -2.909158 2.465174 -0.919594

C -2.291213 4.892139-1.165895

H $-0.8828544 .429671-2.732127$ 
H -0.157956 $5.251884-1.345081$

H $2.8562291 .953403-2.137181$

H $4.1662760 .763077-2.138530$

C $4.7542902 .730840-1.474163$

H $3.3624084 .358345-1.235447$

H $4.9748884 .703537-0.599019$

C $0.8412100 .094652-2.687962$

$\mathrm{H}-3.097850-0.728578-1.006970$

C - $2.576413-0.413347-3.040736$

C - $1.658461-2.207405-1.532801$

$\mathrm{H}-1.788670-2.5198421 .783309$

C -3.898062 -2.918897 1.765732

C -5.343052 -1.045789 1.324704

H -4.378765 0.8437250 .975654

H $3.391412-1.130405-1.981919$

C $4.262734-2.070383-0.279170$

C $2.172382-2.801750-1.480324$

H -0.278966 -1.374986 3.314546

H $0.016021-3.1177173 .278268$

C $1.624870-1.9491814 .150725$

H 1.6641280 .2110054 .113146

H $3.190406-0.5256944 .632055$

$\mathrm{H}-2.9059852 .5309070 .183956$

H -3.650575 $1.694333-1.185128$

C -3.314870 3.814731-1.503477

$\mathrm{H}-2.5821175 .855098-1.616193$

H -2.277733 $5.046855-0.072323$

H $5.6682152 .351318-0.981955$

H $5.0550463 .044655-2.487012$

H $0.304546-0.702523-3.232399$

H $0.5238521 .054490-3.134894$

H $1.899838-0.017577-2.963031$

$\mathrm{H}-1.700756-0.428079-3.709601$

H -2.990863 $0.604444-3.089672$

C -3.622894 -1.402853 -3.551909

C -2.699146 -3.194630 -2.046598

$\mathrm{H}-0.737133-2.290211-2.132373$

$\mathrm{H}-1.375040-2.456058-0.500607$

$\mathrm{H}-3.758074-3.9798881 .990070$

C -5.182121-2.403410 1.599482

H -6.345000 -0.625838 1.201009

H $3.943970-2.5664550 .652849$

H $4.890626-1.2165940 .018433$

C $5.082312-3.055917-1.108463$

C $2.993243-3.787269-2.305442$

H $1.297816-2.454305-2.045301$

H $1.775335-3.312412-0.588637$

H $2.343293-2.7846444 .057044$

H 1.225607 -1.995882 5.176483

H -4.312924 4.098102 -1.131439

H -3.405691 3.725864 -2.601747

H -3.900541 -1.151452 -4.588684

$\mathrm{H}-4.540931-1.292221-2.946936$

C -3.138230 -2.845154 -3.463511

$\mathrm{H}-3.571985-3.175177-1.369740$

H -2.297570 -4.220673 -2.009322 
H $5.954833-3.397385-0.528481$

H $5.483880-2.533755-1.995850$

C $4.241305-4.244375-1.559119$

H $3.291554-3.303566-3.253526$

H $2.370481-4.652961-2.582788$

H -3.926065 -3.535432 -3.806464

$\mathrm{H}-2.284053-2.981974-4.151930$

H $3.938140-4.829219-0.671730$

H $4.840790-4.923010-2.186940$

H -6.054205 -3.055746 1.687288

dimethylamine

$E=-135.053077416$

C -1.206298 $0.216821-0.020118$

$\mathrm{H}-1.2856600 .755742-0.991738$

$\mathrm{H}-2.088427-0.4341610 .077662$

H -1.276742 0.9791320 .774267

C $1.2062970 .216822-0.020117$

H 1.2767940 .9790710 .774322

H $2.088421-0.4341800 .077566$

H $1.2856190 .755813-0.991701$

N -0.000001 -0.5514170 .144760$

H $0.000003-1.343351-0.492286$

\section{3}

$E=-2889.31425265$

Pd $1.663362-0.6117921 .021308$

C 2.9889870 .8365610 .748143

N $2.779734-1.1919662 .798104$

P $0.659071-0.197419-1.041061$

Cl $0.339139-2.5392181 .750010$

P $-2.3106040 .216999-0.061385$

C $-1.0699200 .118129-1.246338$

C - 1.6503320 .3534551 .642003

C $-3.4335471 .650964-0.452585$

C -3.516713 -1.204165 -0.011268

C $1.076367-1.698524-2.075940$

C $1.5809991 .152468-1.972940$

C $3.792935-2.1703272 .416446$

C $3.296565-0.1816853 .709780$

H $2.000054-1.7012413 .226127$

C -1.533382 $0.269700-2.687635$

$\mathrm{H}-1.1264471 .168334-3.185117$

$\mathrm{H}-2.6274490 .354762-2.768780$

$\mathrm{H}-1.258800-0.593677-3.321148$

C - $2.827330-2.5091120 .384339$

C $-4.283456-1.400553-1.323411$

H -4.249115 -0.937506 0.772935

C -5.283986 -2.548296-1.212950

$\mathrm{H}-4.814751-0.482636-1.620781$

H -3.562790 -1.626755 -2.127589

C -4.607269 -3.847222 -0.793829

$\mathrm{H}-6.056209-2.281904-0.468292$

H -5.810286 -2.676558 -2.172963

C -3.820477 -3.659268 0.496550

H -3.915919 -4.168325 -1.593896 
H -5.353010 -4.651486 -0.684806

$\mathrm{H}-4.522680-3.4596941 .327207$

$\mathrm{H}-3.282234-4.5832020 .761719$

$\mathrm{H}-2.072153-2.745720-0.378669$

H -2.253958 -2.405823 1.314847

C -4.511514 1.9312120 .597449

C -2.685001 $2.930625-0.826618$

$\mathrm{H}-3.9554551 .316429-1.367178$

C -3.654059 4.005310-1.308266

$\mathrm{H}-1.9347672 .709793-1.596673$

H -2.125309 3.3071760 .043973

C -4.739815 4.287409-0.276274

$\mathrm{H}-3.1010434 .927551-1.549042$

H -4.125290 3.671345 -2.250749

C -5.475984 3.0109910.113010

H -5.449849 $5.039463-0.656531$

H -4.276079 4.7263220.625908

H -6.033312 $2.629534-0.761811$

H -6.226976 3.2195130.892195

$\mathrm{H}-4.0324562 .2721661 .530825$

H -5.069392 1.0159390 .848258

C -0.9967041 .7054131 .933259$

C - $2.597880-0.0408612 .780443$

H $-0.848591-0.4096121 .622259$

C - 0.2381731 .6503113 .253894

$\mathrm{H}-1.7690572 .4918021 .990752$

$\mathrm{H}-0.3172191 .9785111 .112336$

C - 1.1304241 .2107144 .406946

H 0.5898570 .9299103 .133759

H 0.2206082 .6293573 .469191

C - $1.822005-0.1096494 .093074$

$\mathrm{H}-1.8941701 .9881804 .593345$

$\mathrm{H}-0.5424771 .1267195 .335736$

$\mathrm{H}-2.499915-0.3948494 .913775$

$\mathrm{H}-1.070400-0.9132894 .003839$

$\mathrm{H}-3.4161660 .6930042 .873719$

H -3.064339 -1.016090 2.588779

C $0.147619-2.877295-1.810517$

C $2.542774-2.094503-1.901013$

H $0.927372-1.388679-3.125485$

C $0.520738-4.085027-2.663547$

H $0.192791-3.141698-0.740768$

H -0.889683 -2.571739-2.015164

C $1.978185-4.483271-2.462150$

H -0.145411 -4.930706 -2.425807

H $0.355548-3.848604-3.731328$

C $2.907522-3.309665-2.747661$

H $2.237450-5.344719-3.099306$

H $2.120060-4.810588-1.416398$

H $3.957726-3.592818-2.566686$

H $2.838242-3.042022-3.818152$

H $3.205536-1.248223-2.144391$

H $2.712205-2.323524-0.832580$

C $1.7426231 .036598-3.491844$

C $1.0386132 .526430-1.590160$

H $2.5967921 .083922-1.555237$ 


\begin{abstract}
C $2.6450332 .155859-4.010064$
H $2.1836770 .066793-3.766454$

H $0.7658651 .090088-3.999094$

C $2.1259393 .534939-3.620989$

H $2.7500852 .077311-5.104851$

H $3.6572472 .019926-3.587806$

C $1.9321443 .644176-2.113518$

H $1.1582523 .712091-4.125730$

H $2.8119054 .318905-3.981799$

H $1.5045504 .625833-1.849607$

H $2.9116393 .582608-1.606930$

H $0.0216132 .639239-1.999663$

H $0.9395332 .601809-0.497585$

C 2.8115022 .1273751 .265383

C 4.2303810 .5390660 .161196

C 3.8263813 .0843131 .192815

H 1.8682892 .4007221 .742914

C 5.0504322 .7731890 .602500

H 3.6548594 .0841641 .601596

C 5.2485611 .4920470 .088708

H $6.2026201 .229711-0.377365$

H $4.408563-0.453155-0.263569$

H $4.278193-2.6174763 .303545$

H $4.565056-1.6809391 .805933$

H 2.4933790 .5073204 .001671

H 4.0778500 .4057893 .208599

H $3.315286-2.9657641 .828978$

H $3.723892-0.6433154 .618479$

H 5.8434483 .5225360 .543646
\end{abstract}

\title{
5.3.3 Energies and Coordinates Related to Scheme 3 (RE)
}

Table S13. SCF energies and enthalpy/free energy corrections for Scheme 3. Relative energies are given relative to amido complex 4.

\begin{tabular}{|c|c|c|c|c|c|c|}
\hline & Corr(H) & Corr(G) & $\mathrm{E}(\mathrm{SCF})$ & $\mathrm{E}(\mathrm{PCM})$ & $\Delta \mathbf{G}(\mathrm{PCM}) / \mathrm{E}_{\mathrm{H}}$ & $\begin{array}{c}\Delta \mathrm{G}(\mathrm{PCM}) \\
/ \mathrm{kJ} / \mathrm{mol}\end{array}$ \\
\hline 4 & 1.078127 & 0.947293 & -2428.547501 & -2428.553486 & 0 & 0 \\
\hline $\begin{array}{c}\text { TS } \\
\text { (reductive } \\
\text { elimination) }\end{array}$ & 1.076772 & 0.946509 & -2428.527853 & -2428.533439 & 0.019263 & 50.576 \\
\hline 5 & 1.080013 & 0.947993 & -2428.578865 & -2428.585438 & -0.031252 & -82.052 \\
\hline 1 & 0.993434 & 0.867313 & -2754.187606 & -2754.192664 & -0.037424 & -98.257 \\
\hline $\mathrm{ArNMe}_{2}$ & 0.183965 & 0.140037 & -365.9186969 & -365.9226923 & & \\
\hline
\end{tabular}

4

$E=-2428.55348636$

Pd $1.196485-0.3044831 .220964$

P - $2.172345-0.293833-0.301543$

P $0.7204260 .543931-0.895511$

C - $0.9894510 .361513-1.371505$

C -1.330275 $0.397446-2.853797$

$\mathrm{H}-0.925316-0.458082-3.427628$

H -0.950093 $1.308164-3.343076$ 
H -2.414153 $0.391306-3.041432$

C -3.853192 $0.183339-0.913540$

H -3.920876 -0.349796-1.879102

C -5.017250 -0.313086 -0.052485

$\mathrm{H}-5.0012700 .2066740 .920564$

$\mathrm{H}-4.922501-1.3879830 .164723$

C -6.350014 -0.039902 -0.745857

$\mathrm{H}-6.405138-0.648692-1.666547$

$\mathrm{H}-7.181106-0.373840-0.103978$

C -6.511285 $1.432868-1.104969$

$\mathrm{H}-6.5820162 .025354-0.174730$

H -7.457699 $1.593021-1.646017$

C -5.331202 $1.939557-1.927082$

$\mathrm{H}-5.4399013 .016560-2.132610$

H -5.323905 $1.435333-2.910729$

C -4.006774 1.677018-1.217841

H -3.156364 2.037674 -1.813329

$\mathrm{H}-3.9858112 .247837-0.277392$

C -1.953099 0.249736 1.446777

H -0.8491840 .1666891 .582999$

C -2.594096 -0.6105512 .539568$

$\mathrm{H}-2.376846-1.6768902 .395652$

H -3.691559-0.501704 2.498310

C -2.068551 -0.177107 3.906888

H -2.547477 -0.780547 4.694899

H -0.989577 -0.409998 3.931095

C -2.293689 1.3076034.167492

H -3.377707 1.5050874 .262952

$\mathrm{H}-1.8403881 .5964435 .129383$

C -1.733839 2.166190 3.039028

$\mathrm{H}-0.6329602 .0817713 .018754$

H -1.965759 3.2302053.208228

C -2.283221 1.7220081 .688823

$\mathrm{H}-3.3798901 .8507691 .687698$

$\mathrm{H}-1.8850652 .3416580 .875182$

C -2.258479-2.154698 -0.202040

H $-3.091193-2.4186180 .474440$

C -2.540331-2.749734 -1.585348

H -3.516293 -2.407790 -1.965103

$\mathrm{H}-1.780757-2.375411-2.293181$

C -2.508149 -4.274978 -1.570078

H -2.682239-4.659995 -2.587899

$\mathrm{H}-3.342698-4.648376-0.949168$

C - $1.193626-4.804310-1.011944$

$\mathrm{H}-1.201719-5.905896-0.987831$

H $-0.367727-4.511968-1.685372$

C - $0.931318-4.2392690 .377409$

H $0.039881-4.5887940 .763160$

$\mathrm{H}-1.698042-4.6189661 .077224$

C $-0.956451-2.7149220 .376341$

$\mathrm{H}-0.113112-2.337169-0.223599$

H -0.783291 -2.342103 1.397555

C $1.681807-0.217010-2.306795$

H $1.1896040 .129907-3.233018$

C $3.1512370 .205939-2.386638$

H $3.658077-0.020734-1.437228$ 
H $3.2306931 .293599-2.531961$

C $3.876688-0.506312-3.525145$

H $3.455075-0.179114-4.493815$

H $4.937190-0.206573-3.529069$

C $3.751421-2.020429-3.414358$

H $4.248206-2.355502-2.486107$

H $4.274630-2.515181-4.248957$

C $2.286696-2.437068-3.378108$

H $1.811384-2.179155-4.342405$

H $2.193662-3.530537-3.268738$

C $1.541992-1.737863-2.247329$

H $0.475084-2.009445-2.268499$

H $1.933867-2.086639-1.275570$

C $1.2456692 .326285-0.996919$

H $2.3436892 .258653-0.890605$

C 0.7287213 .1334780 .189294

H -0.3697513.2001170.115835

H 0.9495512 .6026161 .131104

C 1.3118794 .5418770 .202078

H 2.4047454 .4792360 .355116

H 0.9107905 .1128561 .055683

C $1.0257845 .269461-1.107239$

H $1.4916006 .268479-1.105246$

H $-0.0637195 .434935-1.194894$

C $1.5029844 .461381-2.308954$

H $1.2402214 .976932-3.247457$

H $2.6060244 .396065-2.288789$

C $0.9239533 .048293-2.303570$

H $1.3012812 .484045-3.171495$

H $-0.1737273 .102432-2.412557$

C $3.150555-0.1464471 .035305$

C $3.922534-1.2906300 .794693$

C 3.8024131 .0869141 .157114

C $5.306131-1.1967830 .634477$

H $3.443746-2.2706870 .719120$

C 5.1873071 .1782261 .000663

H 3.2300451 .9937701 .368716

C 5.9445420 .0397560 .727665

H $5.888594-2.1005940 .433907$

H 5.6767092 .1521121 .091952

H 7.0269230 .1140780 .598353

N 1.272656-1.024930 3.072994

C $1.726187-2.3743193 .256256$

H $1.323369-3.0384042 .474278$

H $2.834824-2.4775493 .245076$

H $1.386136-2.7773634 .234098$

C $1.789776-0.1692154 .106068$

H $2.901356-0.1367024 .138082$

H 1.4339490 .8654643 .975789

H $1.453911-0.5093135 .109308$

TS (reductive elimination)

$E=-2428.53343902$

Pd $1.230189-0.1194021 .179207$

P - $2.195509-0.417698-0.298802$

P $0.5573380 .813636-0.822415$ 
C -1.133984 $0.507482-1.291946$

C -1.549043 $0.747792-2.734483$

$\mathrm{H}-1.4206971 .798331-3.042612$

$\mathrm{H}-2.6068540 .507844-2.919715$

$\mathrm{H}-0.9788470 .138873-3.461302$

C -3.944171 $-0.003345-0.741753$

H -4.042129 -0.388018-1.773065

C $-5.003438-0.7162660 .101848$

$\mathrm{H}-4.947318-0.3530101 .142135$

H -4.819527 -1.801069 0.138022

C -6.401415 -0.443540 -0.448082

H -6.487574 -0.901402-1.450195

$\mathrm{H}-7.156064-0.9384210 .184340$

C -6.686677 $1.050523-0.550068$

H -6.720554 1.4814230 .467109

H -7.681951 1.220792 -0.991201

C $-5.6119901 .769356-1.358395$

H $-5.8069932 .853611-1.379095$

H -5.651741 $1.427158-2.408663$

C $-4.2193001 .502443-0.796932$

$\mathrm{H}-3.4466982 .010753-1.390550$

$\mathrm{H}-4.1532331 .9250730 .217630$

C -1.912017-0.157611 1.502099

$\mathrm{H}-0.796092-0.2265701 .539933$

C -2.426235 -1.219494 2.477380

H -2.201012 -2.236799 2.131019

H -3.524546 -1.151459 2.560792

C -1.789205 -0.9958973 .848559$

H -2.155099 -1.754530 4.559174

H $-0.698290-1.1420943 .752282$

C -2.062015 0.4069174 .379559

$\mathrm{H}-3.1426810 .5142534 .587556$

$\mathrm{H}-1.5447760 .5569695 .340935$

C - 1.6378951 .4734263 .376529

H -0.5390941 .4508963 .259529$

$\mathrm{H}-1.8984042 .4789263 .744593$

C -2.273193 1.2355822 .011152

H -3.371048 1.3165752 .101522

$\mathrm{H}-1.9498181 .9982521 .292518$

C -2.115756 -2.271421 -0.505278

$\mathrm{H}-2.893453-2.7208260 .138521$

C $-2.402578-2.653916-1.960781$

$\mathrm{H}-3.423116-2.353381-2.247209$

$\mathrm{H}-1.715051-2.091552-2.615808$

C - $2.224105-4.148687-2.207998$

$\mathrm{H}-2.407249-4.373526-3.271217$

$\mathrm{H}-2.988837-4.705122-1.636023$

C - $0.839894-4.625820-1.788405$

H -0.739578 -5.710379-1.955365

$\mathrm{H}-0.078376-4.138997-2.423890$

C - $0.570613-4.280489-0.329959$

H $0.447138-4.588577-0.039531$

$\mathrm{H}-1.263974-4.8524060 .313751$

C - $0.743178-2.789181-0.065873$

H $0.031798-2.226719-0.607529$

H -0.563218 -2.576205 0.998372 
C $1.5877230 .307036-2.296514$ H $1.1450340 .803066-3.180158$ C $3.0483810 .749781-2.183506$ H $3.4800610 .345203-1.254673$ H $3.1176161 .845372-2.106985$ C $3.8754490 .277381-3.376250$ H $3.5237710 .787829-4.292253$ H $4.9269590 .575753-3.235202$ C $3.774983-1.229693-3.574766$ H $4.224521-1.734658-2.701871$ H $4.356284-1.543628-4.457345$ C $2.320749-1.663525-3.708475$ H $1.893479-1.234389-4.633568$ H $2.249701-2.759377-3.813093$ C $1.496895-1.201398-2.512436$ H $0.443465-1.496898-2.633954$ H $1.864515-1.704240-1.600898$ C $0.9011512 .651217-0.770562$ H $1.9832392 .677301-0.540446$ C 0.1805413 .3214400 .394024 H -0.906299 3.280047 0.201173 H 0.3647892 .7526931 .320937 C 0.6079864 .7759030 .560932 H 1.6747434 .8061350 .848050 H 0.0504545 .2435451 .389428 C $0.4108185 .567458-0.727128$ H $0.7721936 .601758-0.605184$ H -0.670939 $5.639785-0.943291$ C $1.1067534 .892848-1.903944$ H $0.9147795 .450364-2.835614$ H $2.2002774 .919393-1.745627$ C $0.6624623 .440059-2.057468$ H $1.1813222 .975923-2.910729$ H -0.415294 $3.418393-2.293403$ C $3.212047-0.4808111 .241319$ C $3.714430-1.6400820 .621618$ C 4.0817120 .6130611 .408121 C $5.025580-1.6834250 .150832$ H $3.067846-2.5084640 .478161$ C 5.3897430 .5591500 .930450 H 3.7231121 .5293341 .882009 C $5.874128-0.5849680 .294361$ H $5.384388-2.591482-0.342339$ H 6.0365411 .4328001 .051347 H $6.899343-0.621667-0.079863$ N $2.047255-0.8919902 .855645$ C $2.182915-2.2964853 .099531$ H $1.726755-2.8834522 .289548$ H $3.241791-2.6211743 .200078$ H $1.659298-2.5757464 .035635$ C $2.559056-0.0803793 .919687$ H $3.645525-0.2337844 .099763$ H 2.3996510 .9868513 .711277 H $2.028314-0.3084314 .865585$ 


$$
E=-2428.58543832
$$

Pd 1.3570880 .5286221 .004314 P - $2.112207-0.724099-0.217280$

P $0.2490911 .111611-0.850434$

C $-1.2952480 .339569-1.287663$

C $-1.7584080 .454195-2.726291$

$\mathrm{H}-1.6878811 .494911-3.090681$

$\mathrm{H}-2.8098940 .159092-2.871661$

$\mathrm{H}-1.170826-0.157136-3.438956$

C $-3.919228-0.695997-0.626773$

$\mathrm{H}-3.938166-1.053855-1.672677$

C $-4.808256-1.6429080 .181189$

$\mathrm{H}-4.827885-1.3187421 .235198$

$\mathrm{H}-4.407516-2.6680010 .175743$

C $-6.233421-1.640829-0.367324$

H -6.224797 -2.063654 -1.388404

H $-6.869643-2.3066920 .238133$

C $-6.821205-0.234747-0.409629$

$\mathrm{H}-6.9403940 .1373400 .624277$

H -7.831495 -0.254955 -0.849398

C $-5.9193520 .722527-1.181085$

$\mathrm{H}-6.3318581 .744080-1.157029$

H -5.892359 $0.424033-2.245025$

C $-4.4993350 .720642-0.626138$

$\mathrm{H}-3.8466571 .397979-1.194803$

$\mathrm{H}-4.5136691 .1066180 .406099$

C - $1.789218-0.3362231 .546863$

$\mathrm{H}-0.665417-0.3210631 .528572$

C $-2.250351-1.3644112 .580791$

$\mathrm{H}-1.994501-2.3914972 .283333$

H -3.349300 -1.333974 2.677603

C - $1.623725-1.0517393 .937832$

$\mathrm{H}-1.972854-1.7774534 .690675$

$\mathrm{H}-0.531233-1.1833503 .856386$

C - 1.9261620 .3726754 .390997

$\mathrm{H}-3.0073670 .4583044 .606123$

$\mathrm{H}-1.4067570 .5894075 .339301$

C -1.547024 1.393977 3.324948

$\mathrm{H}-0.4508421 .3966503 .170390$

$\mathrm{H}-1.8267342 .4102163 .647353$

C -2.1952141.0681731.985014

$\mathrm{H}-3.2936111 .1367392 .077024$

$\mathrm{H}-1.8852721 .7950671 .224174$

C $-1.669860-2.535315-0.354676$

$\mathrm{H}-2.368794-3.1144590 .275664$

C -1.829248 -2.991520 -1.808445

$\mathrm{H}-2.880832-2.903916-2.126511$

$\mathrm{H}-1.251142-2.309962-2.454858$

C -1.343986 -4.420896 -2.025951

$\mathrm{H}-1.441403-4.688694-3.090604$

$\mathrm{H}-1.996016-5.117891-1.468371$

C $0.094019-4.598144-1.556303$

H $0.423098-5.639270-1.705940$

H $0.759983-3.965557-2.170241$

C $0.231746-4.197882-0.093899$

H $1.278049-4.3019460 .236982$ 
H $-0.360368-4.8941340 .528463$

C -0.242945 -2.769565 0.148829

H $0.433797-2.055276-0.347223$

$\mathrm{H}-0.174101-2.5347161 .221708$

C $1.3729930 .724657-2.306212$

H $0.8420921 .058405-3.218140$

C $2.7093531 .463471-2.235515$

H $3.1935931 .207148-1.274337$

H $2.5514402 .552960-2.228695$

C $3.6293971 .092212-3.395975$

H $3.1839071 .450923-4.342334$

H $4.5944311 .615923-3.290917$

C $3.849257-0.412882-3.484258$

H $4.388555-0.750954-2.582239$

H $4.487519-0.660987-4.348704$

C $2.520083-1.153056-3.566455$

H $2.018946-0.903856-4.520138$

H 2.686747 -2.243747 -3.578459

C $1.610070-0.778967-2.402699$

H $0.644971-1.301616-2.482436$

H $2.070436-1.112368-1.454207$

C $0.0233312 .968310-1.134560$

H $0.3027333 .152948-2.189962$

C $0.9694613 .773445-0.239818$

H 0.7051263 .5605990 .812198

H $2.0055583 .419992-0.351849$

C $0.8766385 .270924-0.515843$

H $1.2374575 .473611-1.541153$

H 1.5465385 .8254390 .162531

C $-0.5548855 .777169-0.379307$

$\mathrm{H}-0.6148596 .847957-0.635596$

$\mathrm{H}-0.8663315 .6922430 .677987$

C - $1.5131564 .964533-1.243186$

$\mathrm{H}-2.5504505 .308173-1.093270$

H -1.279626 $5.138942-2.309574$

C - $1.4055283 .471566-0.945882$

H -2.106365 $2.897980-1.568986$

$\mathrm{H}-1.7155323 .2957770 .098173$

C $3.689757-0.7790391 .580546$

C $3.314791-2.0702051 .163032$

C $4.869541-0.2333031 .051408$

C $4.071458-2.7645060 .229411$

H $2.397871-2.5211751 .540003$

C $5.619306-0.9387280 .110875$

H 5.2058480 .7576201 .350466

C $5.231474-2.205924-0.310509$

H $3.742036-3.757168-0.087504$

H $6.525179-0.480254-0.293254$

H $5.821658-2.753261-1.047974$

N $2.881662-0.0627392 .521535$

C $2.357263-0.8720223 .618382$

H $1.765491-1.7118833 .240786$

H $3.172753-1.2655374 .256388$

H $1.692119-0.2480854 .228883$

C 3.4646921 .1721013 .034176

H 4.3950520 .9819993 .606459 
H 3.6745501 .8648872 .210756

H 2.7310231 .6549553 .691420

dimethylphenylamine

$E=-365.922692334$

C -0.1881410 .0000080 .000591$

C $0.545356-1.2074680 .000545$

C $0.5453661 .207479-0.000067$

C $1.935875-1.1969790 .000181$

H $0.026669-2.1657620 .000882$

C $1.9358851 .196979-0.000407$

H $0.0266892 .165778-0.000398$

C $2.649765-0.000004-0.000302$

H 2.469694 -2.151070 0.000204

H $2.4697132 .151064-0.000910$

H $3.741421-0.000008-0.000652$

$\mathrm{N}-1.5649240 .0000040 .001196$

C -2.281721 -1.245151-0.001024

H - 2.055382 -1.858251-0.893075

$\mathrm{H}-2.056119-1.8607770 .889436$

$\mathrm{H}-3.360218-1.044310-0.001100$

C -2.281745 1.2451370.000066

$\mathrm{H}-2.0542541 .8608760 .889933$

$\mathrm{H}-2.0573461 .858147-0.892576$

H -3.360238 1.0442840 .002388 


\subsubsection{Energies and Coordinates Related to Figure 6}

Table S14. SCF energies and enthalpy/free energy corrections for the BHA with L2 and L3 (Figure 6). Relative energies are given relative to the arene complexes

\begin{tabular}{|c|c|c|c|c|c|c|}
\hline $\begin{array}{c}\text { L2 }= \\
\text { CyJohnPhos }\end{array}$ & Corr(H) & Corr(G) & $E(S C F)$ & $\mathrm{E}(\mathrm{PCM})$ & $\underset{\mathrm{E}_{\mathrm{H}}}{\Delta \mathrm{G}(\mathrm{PCM}) /}$ & $\begin{array}{c}\Delta G_{M}- \\
(P C M) / k J / \\
\mathrm{mol}\end{array}$ \\
\hline $\begin{array}{c}\text { L2-Pd(Ar)- } \\
\mathrm{NMe}_{2}\end{array}$ & 0.706682 & 0.604333 & -1766.428605 & -1766.435468 & 0.083900 & 228.19 \\
\hline TS (RE) & 0.705397 & 0.603231 & -1766.412589 & -1766.419239 & 0.099027 & 267.91 \\
\hline L2-Pd-ArNMe 2 & 0.708246 & 0.606966 & -1766.465697 & -1766.472336 & 0.049665 & 138.31 \\
\hline L2-Pd-ArCl & 0.622412 & 0.524165 & -2092.071833 & -2092.078714 & 0.042220 & 118.76 \\
\hline TS (OA) & 0.620868 & 0.522851 & -2092.052915 & -2092.059864 & 0.059756 & 163.80 \\
\hline L2-Pd(Ar)-Cl & 0.623903 & 0.527819 & -2092.097159 & -2092.114341 & 0.010247 & 34.81 \\
\hline $\begin{array}{c}1 / 2[\mathrm{~L} 2-\mathrm{Pd}(\mathrm{Ar})- \\
\mathrm{Cl}]_{2}\end{array}$ & & 0.540476 & & -2092.137245 & 0.000000 & 0.00 \\
\hline $\begin{array}{l}\text { Rotation (from } \\
\text { PES Scan) }\end{array}$ & & & & -2092.09375 & 0.030815 & 88.81 \\
\hline $\begin{array}{c}\text { L2-Pd(Ar)-Cl- } \\
\text { distal }\end{array}$ & 0.624061 & 0.526221 & -2092.084268 & -2092.105109 & 0.017881 & 54.86 \\
\hline $\begin{array}{l}\text { TS cis-trans- } \\
\text { isomerization } \\
\text { (distal) }\end{array}$ & 0.623061 & 0.527792 & -2092.081605 & -2092.099103 & 0.025458 & 74.75 \\
\hline $\begin{array}{c}\text { L2-Pd(Ar)-Cl - } \\
\text { o (distal) }\end{array}$ & 0.624267 & 0.526123 & -2092.080486 & -2092.10004 & 0.022852 & 67.91 \\
\hline $\begin{array}{l}\mathrm{L} 2-\mathrm{Pd}(\mathrm{Ar})-\mathrm{Cl}- \\
\mathrm{HNMe}_{2} \text { (distal) }\end{array}$ & 0.725815 & 0.618113 & -2227.187041 & -2227.198023 & 0.002756 & 7.23 \\
\hline \multicolumn{7}{|l|}{$\mathbf{L} 3=\mathrm{PtBu}_{3}$} \\
\hline $\begin{array}{c}\text { L3-Pd(Ar)- } \\
\mathrm{NMe}_{2}\end{array}$ & 0.574313 & 0.485172 & -1307.047417 & -1307.05489 & 0.066379 & 182.19 \\
\hline TS (RE) & 0.573296 & 0.486758 & -1307.032579 & -1307.038278 & 0.084577 & 229.97 \\
\hline L3-Pd-ArNMe 2 & 0.576829 & 0.486307 & -1307.085729 & -1307.091726 & 0.030678 & 88.45 \\
\hline L3-Pd-ArCl & 0.490072 & 0.405493 & -1632.694139 & -1632.698849 & 0.024475 & 72.17 \\
\hline TS (OA) & 0.489164 & 0.40769 & -1632.676202 & -1632.681368 & 0.044153 & 123.83 \\
\hline L3-Pd(Ar)-Cl & 0.49127 & 0.408903 & -1632.708143 & -1632.726115 & 0.000619 & 9.53 \\
\hline $\begin{array}{c}1 / 2[\mathrm{~L} 3-\mathrm{Pd}(\mathrm{Ar})- \\
\mathrm{Cl}]_{2}\end{array}$ & 0.422996 & & -1632.740826 & & 0.000000 & 0.00 \\
\hline $\begin{array}{l}\text { TS cis-trans- } \\
\text { isomerization }\end{array}$ & 0.490489 & 0.40964 & -1632.70275 & -1632.718381 & 0.009089 & 31.77 \\
\hline $\begin{array}{c}\text { L3-Pd(Ar)-Cl - } \\
0\end{array}$ & 0.491569 & 0.409614 & -1632.703519 & -1632.719022 & 0.008423 & 30.03 \\
\hline $\begin{array}{c}\text { L3-Pd(Ar)-Cl- } \\
\mathrm{HNMe}_{2}\end{array}$ & 0.593337 & 0.50085 & -1767.796489 & -1767.80525 & -0.000672 & -1.77 \\
\hline
\end{tabular}




\section{Structures of the dimeric oxidative addition product $[\mathrm{L2}-\mathrm{Pd}(\mathrm{Ar})-\mathrm{Cl}]_{2}$}

There are four possible structures (A-D) for the OA-dimer of L2, which differ in the position of the phosphine at the $\mathrm{Pd}_{2} \mathrm{Cl}_{2}$ ring (cis and trans) and in the orientation of the biaryl moiety (either on the same or opposite side of the $\mathrm{Pd}_{2} \mathrm{Cl}_{2}$ ring)

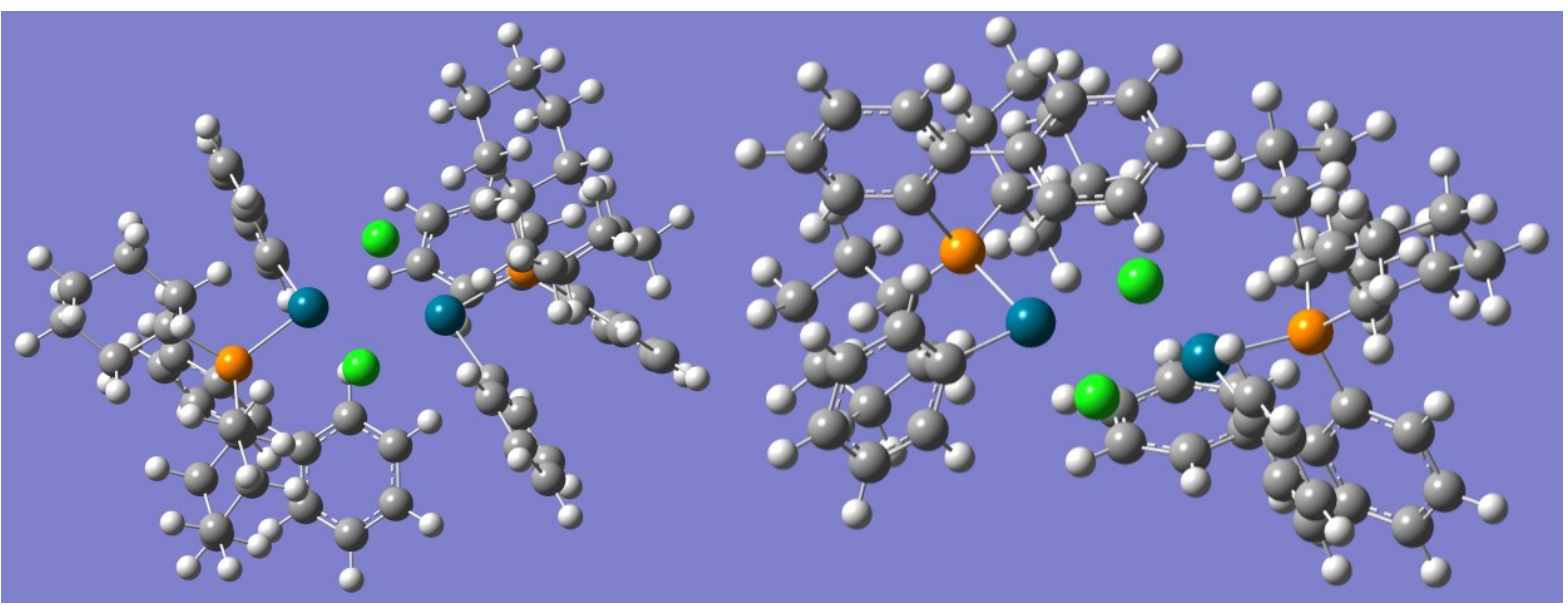

A (trans, L2 same side)

B (cis, L2 different sides)

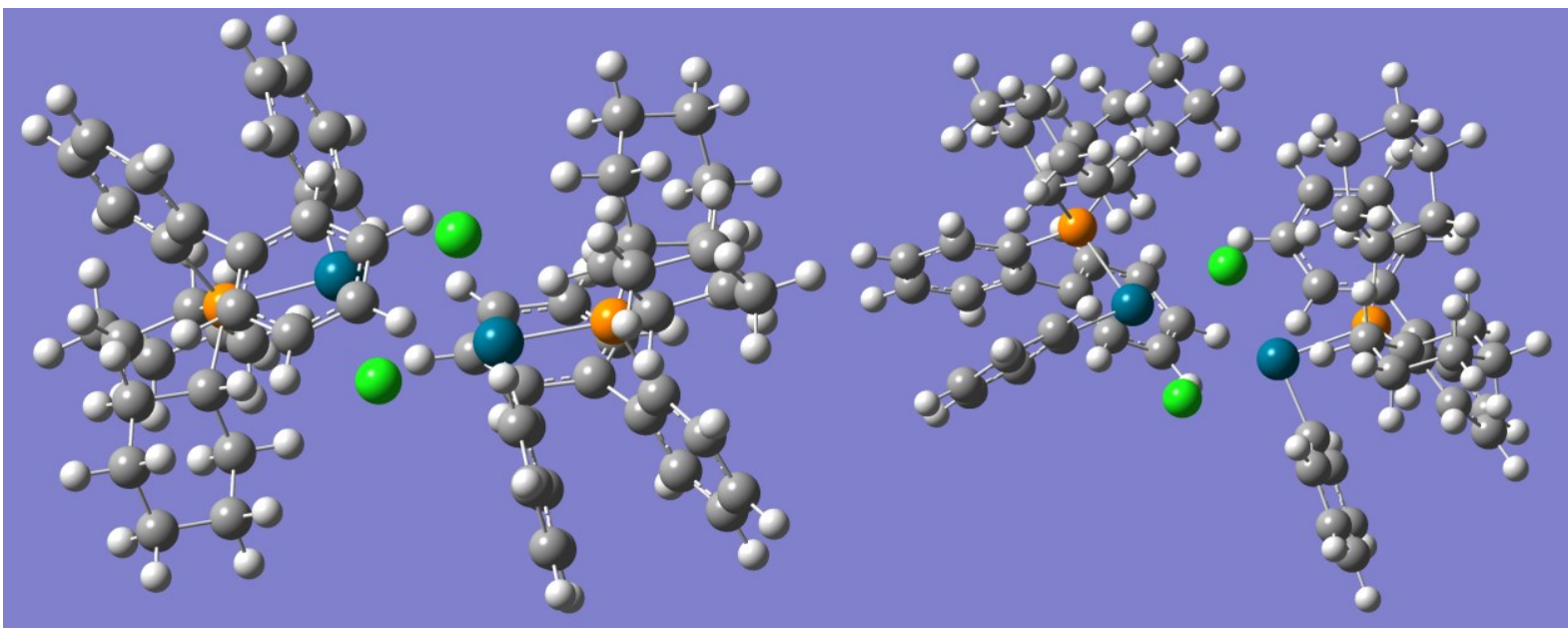

C (trans, L2 different sides)

D (cis, L2 same sides)

Table S15. Energies of structures A-D.

\begin{tabular}{ccccccc}
\hline & Corr(H) & Corr(G) & $\mathbf{E}(\mathbf{S C F})$ & $\mathbf{E}(\mathbf{P C M})$ & $\boldsymbol{\Delta G}(\mathbf{P C M}) / \mathbf{E}_{\mathrm{H}}$ & $\boldsymbol{\Delta} \mathbf{G}(\mathbf{P C M}) / \mathbf{k J} / \mathbf{m o l}$ \\
\hline \hline A & 1.250858 & 1.082106 & -4184.258331 & -4184.273885 & 0.001759 & 4.62 \\
B 1.250414 & 1.080952 & -4184.258316 & -4184.274490 & 0.000000 & 0.00 \\
C 1.250524 & 1.079357 & -4184.254812 & -4184.270609 & 0.002286 & 6.00 \\
D 1.250782 & 1.079984 & -4184.252487 & -4184.268848 & 0.004674 & 12.27 \\
\hline
\end{tabular}


Table S16. Energies for rotation around the Pd-P-C-C bond for L2 in cis-2 (Figure 6).

\begin{tabular}{|c|c|c|c|}
\hline$\circ$ & E(PCM) & $\Delta G(P C M) / E_{H}$ & $\Delta G(P C M) / k J / m o l$ \\
\hline-170 & -2092.114355 & 0.0000 & 0.0 \\
\hline-160 & -2092.113012 & 0.0013 & 3.5 \\
\hline-150 & -2092.111698 & 0.0026 & 6.9 \\
\hline-140 & -2092.111008 & 0.0033 & 8.7 \\
\hline-130 & -2092.110074 & 0.0043 & 11.2 \\
\hline-120 & -2092.107452 & 0.0069 & 18.1 \\
\hline-110 & -2092.102897 & 0.0114 & 30.0 \\
\hline-100 & -2092.097844 & 0.0165 & 43.3 \\
\hline-90 & -2092.095074 & 0.0193 & 50.6 \\
\hline-80 & -2092.092367 & 0.0220 & 57.7 \\
\hline-70 & -2092.087604 & 0.0267 & 70.2 \\
\hline-60 & -2092.091845 & 0.0225 & 59.1 \\
\hline-50 & -2092.095611 & 0.0187 & 49.2 \\
\hline-40 & -2092.098338 & 0.0160 & 42.0 \\
\hline-30 & -2092.099039 & 0.0153 & 40.2 \\
\hline-20 & -2092.099385 & 0.0150 & 39.3 \\
\hline-10 & -2092.098612 & 0.0157 & 41.3 \\
\hline 0 & -2092.102537 & 0.0118 & 31.0 \\
\hline 10 & -2092.09855 & 0.0158 & 41.5 \\
\hline 20 & -2092.101658 & 0.0127 & 33.3 \\
\hline 30 & -2092.104471 & 0.0099 & 25.9 \\
\hline 40 & -2092.105244 & 0.0091 & 23.9 \\
\hline 50 & -2092.104546 & 0.0098 & 25.7 \\
\hline 60 & -2092.101905 & 0.0124 & 32.7 \\
\hline 70 & -2092.097439 & 0.0169 & 44.4 \\
\hline 80 & -2092.093749 & 0.0206 & 54.1 \\
\hline 90 & -2092.094978 & 0.0194 & 50.8 \\
\hline 100 & -2092.095216 & 0.0191 & 50.2 \\
\hline 110 & -2092.095601 & 0.0187 & 49.2 \\
\hline 120 & -2092.100464 & 0.0139 & 36.4 \\
\hline 130 & -2092.103648 & 0.0107 & 28.1 \\
\hline 140 & -2092.104948 & 0.0094 & 24.7 \\
\hline 150 & -2092.10536 & 0.0090 & 23.6 \\
\hline 160 & -2092.107961 & 0.0064 & 16.7 \\
\hline 170 & -2092.111152 & 0.0032 & 8.4 \\
\hline 180 & -2092.11365 & 0.0007 & 1.8 \\
\hline Eq (-171.8) & -2092.114341 & & 0.0 \\
\hline
\end{tabular}

L2 $=$ Cy JohnPhos

L2-Pd(Ar)-NMe 2 $E=-1766.43546799$

Pd $0.0108701 .215729-0.156817$

C $0.958916-1.9706261 .165221$

H $0.544851-2.9875741 .297813$

C $2.362933-2.1197870 .573293$

H $2.761885-1.1288700 .308031$

H $2.321724-2.707764-0.356811$ 
C $3.309705-2.7974241 .559981$

H $2.984018-3.8410031 .727139$

H $4.318395-2.8512701 .120048$

C $3.347462-2.0648122 .895051$

H $3.756090-1.0514572 .735781$

H $4.028559-2.5749233 .595432$

C $1.950350-1.9565253 .493382$

H $1.577859-2.9690563 .735087$

H $1.976442-1.4013374 .445073$

C $-0.253961-2.146986-1.481926$

H $0.065809-3.152562-1.146669$

C $0.750134-1.627389-2.513864$

H $0.436174-0.610320-2.809538$

H $1.749234-1.512239-2.070626$

C $0.808933-2.528725-3.742016$

H $1.206498-3.517113-3.447483$

H $1.520795-2.115595-4.474710$

C $-0.566931-2.704727-4.372936$

H - $0.516235-3.399665-5.226671$

H $-0.903002-1.734436-4.781436$

C - $1.584711-3.192380-3.348442$

H -2.586508 $-3.263996-3.802114$

$\mathrm{H}-1.317343-4.214772-3.024917$

C -1.635330 -2.282200 -2.124677

$\mathrm{H}-2.371783-2.657445-1.398775$

$\mathrm{H}-1.983334-1.282301-2.438395$

C $1.9955231 .249422-0.226788$

C 2.7525981 .4568410 .934212

C $2.6842441 .073934-1.434284$

C 4.1481801 .4331220 .900833

H 2.2529641 .6425791 .888419

C $4.0802091 .049864-1.470872$

H $2.1300240 .951706-2.367568$

C $4.8204341 .215641-0.301083$

H 4.7129781 .5894981 .824470

H $4.5920880 .901939-2.426112$

H $5.9125001 .191756-0.328574$

N $0.0688143 .214522-0.265967$

C 0.6129273 .9414770 .842702

H 0.2610293 .5191121 .798294

H 1.7252173 .9743990 .876826

H 0.2717344 .9983120 .810637

C $0.4866263 .778384-1.517023$

H $1.5860583 .752222-1.691157$

H $0.0049623 .257977-2.361626$

H $0.1849524 .846023-1.578114$

P $-0.225964-1.0748280 .046357$

C - $1.821833-1.4351950 .884380$

C - $2.118811-2.7223401 .354049$

C -2.724519 -0.3852501 .136302$

C -3.268439-2.974752 2.093816

$\mathrm{H}-1.429678-3.5455051 .148931$

C -3.872855 -0.650871 1.899687

C -4.142488 -1.926943 2.380355

H -3.477595 -3.984877 2.453392

H - 4.5730520 .1651202 .093202 
H -5.045170 -2.107080 2.968899

C -2.581310 0.9916220 .583092

C -2.658376 2.110099 1.435995

C -2.656865 1.206091-0.810065

C -2.773636 3.3893260 .911578

H -2.616335 1.9566282 .516902

C $-2.8151772 .491366-1.327786$

$\mathrm{H}-2.6826690 .342570-1.476567$

C $-2.8428003 .584225-0.469337$

$\mathrm{H}-2.7967004 .2496451 .584070$

H -2.885639 2.633685 -2.408054

H -2.930993 $4.594974-0.872956$

C $0.981520-1.2794242 .530280$

H -0.033673 -1.261255 2.958148

H $1.278737-0.2288402 .383600$

TS (reductive elimination)

$E=-1766.41923919$

Pd $0.401491-1.0616160 .210845$

C $0.1129722 .139031-1.211711$

H $-0.5922452 .982088-1.337024$

C $1.4302162 .694147-0.664464$

H $2.1016821 .858482-0.408342$

H 1.2491283 .2557080 .265103

C $2.1167723 .604563-1.678779$

H $1.5031534 .511598-1.833322$

H $3.0814603 .944187-1.268973$

C $2.3237232 .905550-3.016312$

H $3.0191112 .060761-2.872544$

H $2.7990253 .589192-3.738515$

C $1.0039332 .380075-3.567219$

H $0.3386943 .231863-3.800463$

H $1.1654541 .844668-4.517061$

C - 0.9962231 .9315221 .495436

$\mathrm{H}-1.0528252 .9900521 .175965$

C 0.1712511 .7654082 .474011

H 0.2376820 .6945582 .738675

H 1.1303862 .0065021 .994045

C - 0.0265902 .6002483 .733932

H -0.011186 3.672769 3.466990

H 0.8184132 .4417444 .423338

C - 1.3462902 .2686464 .419829

$\mathrm{H}-1.4993082 .9126435 .300975$

$\mathrm{H}-1.3054531 .2304504 .796552$

C -2.517223 2.4017853 .453310

$\mathrm{H}-3.4594532 .1078773 .943853$

$\mathrm{H}-2.6351323 .4628243 .167313$

C -2.309673 1.5696012 .191172

$\mathrm{H}$-3.162862 1.6950361 .508134

$\mathrm{H}-2.2822000 .4993572 .464582$

C $2.397513-0.7688000 .202046$

C $3.070892-0.606564-1.027605$

C $3.009760-0.2282781 .352727$

C $4.2720190 .095398-1.102562$

H $2.639227-1.014636-1.943815$

C 4.2069560 .4782071 .264248 
H $2.527952-0.3334702 .326723$

C 4.8502180 .6548740 .037678

H $4.7592960 .210433-2.075353$

H 4.6399700 .9031432 .174599

H $5.7887211 .209318-0.026384$

N $1.674467-2.6240750 .431190$

C $2.201101-3.398742-0.650978$

H $1.740968-3.107260-1.605614$

H $3.304566-3.302147-0.759053$

H $1.973407-4.471425-0.499601$

C $2.114621-3.0665511 .719298$

H $3.216659-2.9931631 .853586$

H $1.640804-2.4805782 .519636$

H $1.826472-4.1241721 .881602$

P $-0.6729120 .927556-0.043841$

C -2.330143 0.711773 -0.812785

C -3.070134 1.822776-1.241487

C $-2.831588-0.582040-1.059472$

C -4.272869 1.674243-1.922915

$\mathrm{H}-2.6912172 .829509-1.046304$

C $-4.037912-0.717790-1.764335$

C -4.752651 $0.393963-2.195651$

H -4.831134 2.555491-2.247386

H -4.422142 -1.722573 -1.955893

$\mathrm{H}-5.6916780 .260611-2.737986$

C -2.174462 -1.833753 -0.587520

C - $1.768384-2.813106-1.508442$

C - $2.095618-2.1325380 .785888$

C -1.292230 -4.046109-1.070600

$\mathrm{H}-1.824563-2.592085-2.576960$

C -1.636582 -3.3777631 .218514$

$\mathrm{H}-2.453871-1.3996871 .511163$

C $-1.227053-4.3328170 .292274$

$\mathrm{H}-0.964472-4.788765-1.801683$

$\mathrm{H}-1.588226-3.5916652 .288376$

H - $0.851332-5.3002870 .632124$

C $0.3125351 .459208-2.568100$

$\mathrm{H}-0.6571961 .118870-2.965929$

H $0.9249550 .554699-2.411263$

L2-Pd(ArNMe 2 )

$E=-1766.47233642$

Pd $0.028342-1.1032450 .825911$

C $0.0778461 .386518-1.815838$

H $-0.5599492 .182077-2.243069$

C $1.4859811 .942015-1.609825$

H $2.0813581 .206095-1.044076$

H $1.4497032 .855249-0.996174$

C $2.1837352 .250356-2.931368$

H $1.6677763 .090716-3.431043$

H $3.2130242 .590123-2.730441$

C $2.1924321 .046427-3.864541$

H $2.8021950 .245066-3.410284$

H $2.6741621 .304053-4.822026$

C $0.7792160 .526633-4.096642$

H $0.1916341 .292193-4.635357$ 
H $0.794486-0.364481-4.745487$

C -0.7937062 .3491270 .839809$

$\mathrm{H}-0.8699153 .2214980 .161993$

C 0.4773302 .4814981 .685680

H 0.5565171 .5708072 .307240

H 1.3762782 .4866691 .053420

C 0.4442463 .7172892 .577944

H 0.4383924 .6243371 .946095

H 1.3651373 .7682713 .182204

C - 0.7856513 .7200623 .477160

$\mathrm{H}-0.8233004 .6403674 .082643$

H -0.7112842 .8796774 .191052$

C -2.061717 3.5660452 .658527

$\mathrm{H}-2.9414063 .5140633 .320686$

$\mathrm{H}-2.2008354 .4643012 .029578$

C -2.017328 2.3341241.759542

$\mathrm{H}-2.9446502 .2609081 .173115$

H - 1.9722391 .4243272 .385766

C $3.095814-0.9897390 .399629$

C $3.859219-0.927713-0.771832$

C 3.2338850 .0438331 .341154

C $4.7206100 .148449-1.000494$

H $3.785569-1.712232-1.523757$

C 4.0994201 .1033761 .112373

H 2.6162300 .0307942 .240918

C $4.8501501 .167732-0.065208$

H $5.2963210 .179552-1.928785$

H 4.1750621 .9010681 .855394

H $5.5230672 .007824-0.248726$

N 2.182401-2.052977 0.663506

C $1.999011-3.005999-0.421266$

H $1.714126-2.478897-1.340431$

H $2.911445-3.605870-0.612090$

H $1.174620-3.682381-0.157380$

C $2.466450-2.7572481 .914468$

H $3.439239-3.2847371 .865534$

H $2.488676-2.0587732 .758707$

H $1.667370-3.4866072 .098676$

P $-0.6877490 .808732-0.214928$

C -2.439496 $0.587432-0.786914$

C -3.074794 1.651511-1.444037

C -3.134123 -0.641407-0.655873

C -4.355154 1.530281-1.973059

$\mathrm{H}-2.5525722 .606958-1.546921$

C $-4.419793-0.752137-1.211725$

C -5.027906 0.314079-1.862416

$\mathrm{H}-4.8250592 .381545-2.471477$

H -4.951001 -1.700505-1.101609

$\mathrm{H}-6.0337620 .200034-2.274031$

C -2.539408 -1.815071 0.019005

C -2.471378 -3.048476 -0.604874

C - $1.943583-1.6390221 .317630$

C -1.811525 -4.142245 0.001926

$\mathrm{H}-2.901865-3.162671-1.603514$

C - $1.217999-2.7272291 .892853$

H -2.324983 -0.831298 1.951030 
C -1.184705 -3.977786 1.221801

$\mathrm{H}-1.790226-5.110141-0.504037$

$\mathrm{H}-0.923134-2.6795622 .944993$

H $-0.679927-4.8247121 .696239$

C $0.0897160 .196710-2.778517$

H - $0.939398-0.155005-2.955537$

H $0.618013-0.638141-2.281965$

L2-Pd(ArCl)

$E=-2092.07871413$

Pd $1.007227-0.068444-0.554880$

$\mathrm{P}-1.196759-0.2086250 .080758$

C -1.494042 -1.297361 1.564553

$\mathrm{H}-2.527487-1.1186831 .914273$

C -1.336174 -2.785431 1.257129

$\mathrm{H}-0.362545-2.9426760 .762848$

$\mathrm{H}-2.111718-3.1117400 .547020$

C $-1.415066-3.6386682 .520608$

$\mathrm{H}-2.436238-3.5733832 .938650$

H - $1.253960-4.6984282 .263329$

C $-0.415603-3.1841643 .576679$

H $0.610378-3.3333353 .194974$

H - $0.506921-3.8028954 .484130$

C - $0.611415-1.7101473 .907641$

$\mathrm{H}-1.601406-1.5698504 .378976$

H $0.134878-1.3765784 .646812$

C $-0.515284-0.8477212 .654982$

H -0.691922 0.2115992 .902517

H $0.509125-0.9039192 .243180$

C - $2.415528-0.750271-1.221806$

H $-3.330399-1.116515-0.717500$

C $-1.812632-1.886165-2.057574$

$\mathrm{H}-0.863481-1.519424-2.488974$

$\mathrm{H}-1.539920-2.741424-1.423389$

C - $2.752175-2.333415-3.172369$

H -3.664085 -2.773152 -2.728466

H -2.277034 -3.135237 -3.760499

C -3.140747 -1.168590 -4.073957

H -3.853558 -1.497087 -4.847629

H - $2.242078-0.811643-4.608720$

C -3.726322 -0.020553 -3.261343

H -3.954862 $0.838294-3.913175$

H -4.688466 -0.339150 -2.820414

C -2.788624 $0.417244-2.140613$

H -3.243956 1.236394 -1.564662

$\mathrm{H}-1.8583670 .822665-2.580512$

Cl $3.445238-0.190294-2.565637$

C $3.026913-0.673708-0.915579$

C $3.811398-0.1623400 .155373$

C $2.265914-1.870989-0.724159$

C $3.846994-0.8255931 .365538$

H $4.3937000 .745327-0.004591$

C $2.398696-2.5621110 .511552$

H $1.869545-2.402483-1.592568$

C $3.161794-2.0482931 .538769$

H $4.437137-0.4090692 .185066$ 
H $1.902828-3.5293130 .625515$

H $3.248809-2.5919432 .482268$

C - 1.9429131 .3498330 .730626

C -3.281077 1.347860 1.150434

C - 1.1731042 .5178840 .913473

C -3.8620012.4618321.745597

$\mathrm{H}-3.8850240 .4465621 .012488$

C - 1.7674203 .6293581 .534246

C -3.094227 3.608032 1.946382

$\mathrm{H}-4.9088792 .4338792 .056895$

$\mathrm{H}-1.1690024 .5333701 .669592$

H -3.532815 4.4912612.416795

C 0.2398992 .6467860 .477592

C 1.2223763 .0627181 .380829

C $0.6055032 .426608-0.868440$

C 2.5363913 .2771990 .960257

H 0.9522233 .2128452 .428902

C $1.9221942 .651642-1.285968$

H -0.170458 2.211184-1.606226

C $2.8859373 .080555-0.372318$

H 3.2886663 .6021901 .682842

H $2.1879022 .498336-2.333057$

H $3.9113353 .254643-0.706529$

TS (oxidative addition)

$E=-2092.05986444$

Pd -0.393299 $1.344085-0.084493$

P $0.357230-0.816657-0.170870$

C - $0.503664-2.008201-1.295944$

H $0.029566-2.976383-1.290823$

C $-1.936549-2.227178-0.810341$

$\mathrm{H}-2.421912-1.244346-0.691979$

$\mathrm{H}-1.940872-2.6928580 .187817$

C $-2.748077-3.076243-1.782194$

$\mathrm{H}-2.336824-4.102055-1.810296$

$\mathrm{H}-3.783603-3.164974-1.415318$

C -2.728563 -2.488546 -3.187852

H -3.237634 -1.507814 -3.173656

$\mathrm{H}-3.297432-3.127627-3.882603$

C -1.298999-2.301156 -3.682283

$\mathrm{H}-0.816098-3.289817-3.787362$

$\mathrm{H}-1.292316-1.845244-4.685749$

C $-0.488280-1.442373-2.718202$

H $0.550352-1.337171-3.072722$

H -0.915656 -0.423072 -2.684069

C $0.348980-1.6151851 .504521$

$\mathrm{H}-0.722963-1.5457791 .773067$

C $1.121551-0.7345072 .488497$

H $2.190406-0.7317552 .205911$

H 0.7747780 .3090962 .398698

C $0.970749-1.2402453 .918969$

$\mathrm{H}-0.083500-1.1248974 .230700$

H $1.566037-0.6167264 .605565$

C $1.373226-2.7057954 .043428$

H $1.213098-3.0639285 .073385$

H $2.456575-2.8007053 .846146$ 
C $0.610298-3.5778473 .052322$

H $0.942436-4.6263263 .126123$

H -0.463948 -3.572682 3.311973

C $0.780591-3.0740251 .620349$

H $0.208754-3.7100250 .924901$

H $1.843090-3.1626771 .336671$

Cl -1.490671 3.104459 1.303073

C -2.365251 1.5112240.789116

C $-3.2324781 .623834-0.318119$

C -2.667680 0.600167 1.825215

C -4.293831 $0.730441-0.446879$

$\mathrm{H}-3.0560762 .399981-1.065354$

C -3.738397 -0.268071 1.676774

$\mathrm{H}-2.0407300 .5779782 .718626$

C -4.555562 -0.2205340.539635

$\mathrm{H}-4.9386350 .797351-1.327202$

H -3.945739 -0.9944642 .467277$

H -5.400909 -0.903811 0.438493

C $2.115529-0.914183-0.719258$

C $2.721416-2.154538-0.968695$

C $2.8979890 .257708-0.836145$

C $4.072263-2.257611-1.286183$

H $2.123176-3.065835-0.911772$

C $4.2632480 .136625-1.136185$

C $4.850898-1.104701-1.354277$

H 4.515717 -3.238034 -1.475484

H $4.8608941 .047094-1.223938$

H $5.914577-1.169286-1.595528$

C $2.3469781 .632368-0.698694$

C $1.3593482 .095906-1.600447$

C 2.8519622 .5159220 .259214

C $0.9050023 .420557-1.526433$

H $1.0548571 .456536-2.432564$

C 2.3800413 .8288180 .339424

H 3.6086322 .1607620 .962916

C $1.4086934 .282286-0.548224$

H $0.1693823 .777585-2.250545$

H 2.7778644 .5001241 .103845

H $1.0407845 .308421-0.484287$

L2-Pd-Ar-Cl = cis-2

$E=-2092.11434107$

Pd $0.630278-1.240604-0.255128$

C - 1.6038200 .9827901 .395294

$\mathrm{H}-1.6393462 .0577921 .649105$

C -2.997711 0.5742810 .912631

$\mathrm{H}-2.981637-0.4649000 .555096$

$\mathrm{H}-3.3069841 .1963170 .058336$

C -4.020043 0.7007692 .038372

$\mathrm{H}-4.1328391 .7643472 .319646$

H -5.005405 0.3672801 .675675

C -3.602867 -0.101270 3.265052

H -3.575720 -1.173166 3.000180

$\mathrm{H}-4.3495510 .0067284 .068221$

C -2.225993 0.329592 3.756152

H -2.274673 1.3765234.107798 
$\mathrm{H}-1.915785-0.2747004 .623759$

C -1.181667 0.2155102 .651524

$\mathrm{H}-0.2038400 .5822083 .004847$

$\mathrm{H}-1.038641-0.8439382 .382770$

C $-0.9382301 .604510-1.409980$

$\mathrm{H}-1.8072140 .957837-1.632487$

C $0.0438281 .471181-2.574390$

H $0.9444772 .074057-2.355112$

H $0.3773220 .424039-2.664854$

C $-0.5832261 .952700-3.878571$

$\mathrm{H}-1.4145001 .275627-4.145929$

H $0.1501081 .880505-4.697600$

C -1.111571 3.377482 -3.756612

$\mathrm{H}-1.6024223 .687217-4.693111$

$\mathrm{H}-0.2610894 .067570-3.608759$

C -2.073283 $3.514967-2.581817$

$\mathrm{H}-2.4095864 .559261-2.478589$

H -2.979398 $2.913387-2.776173$

C - $1.4320493 .044227-1.278311$

H -2.149339 $3.141677-0.447538$

H - $0.5767423 .700300-1.043337$

Cl $1.369721-3.423172-0.587094$

C -1.191106 -1.970222 -0.466679

C - $1.756785-2.7490920 .548154$

C - $1.920209-1.742811-1.636622$

C -3.055664 -3.240338 0.415817

$\mathrm{H}-1.178376-2.9856081 .443391$

C -3.220229 -2.238836-1.766754

$\mathrm{H}-1.477306-1.181615-2.463152$

C -3.798755 -2.975269 -0.735257

H -3.487256 -3.843025 1.219659

H -3.780791 -2.047013 -2.685765

H $-4.816950-3.358659-0.834974$

P -0.2692860 .8103430 .119664$

C 1.0374271 .8998260 .803986

C 0.7371933 .1804201 .293407

C 2.3423001 .3951600 .957304

C 1.7020243 .9576351 .924012

H -0.276506 3.5733311 .202011

C 3.3016992 .1861271 .608990

C 2.9906523 .4532902 .087936

H 1.4435094 .9508872 .297787

H 4.3137211 .7911121 .723533

H 3.7571214 .0490422 .589062

C 2.8091500 .0764580 .440186

C $3.388882-0.8563581 .325182$

C $2.965674-0.131379-0.947316$

C $4.113317-1.9358550 .840552$

H $3.270340-0.7082172 .401047$

C $3.715556-1.209608-1.424222$

H $2.5809410 .615099-1.644214$

C $4.288443-2.108588-0.534327$

H $4.538357-2.6593321 .539034$

H $3.831984-1.348562-2.500459$

H $4.857663-2.960647-0.909534$ 
cis-trans isomerization

$E=-2092.10210726$

Cl $0.081893-3.682783-1.217943$

Pd $-0.143477-1.642152-0.098303$

H $2.436574-1.658961-1.305661$

C $3.009726-0.715031-1.259547$

P $0.7523310 .404378-0.050637$

C $-1.032446-1.2315151 .589926$

C $2.5746260 .072437-0.018984$

C $4.501735-1.027762-1.214662$

H $2.763082-0.156409$-2.175974

C 0.4008481 .5901951 .327655

C -2.401668 -0.954973 1.549026

C - $0.393505-1.4129582 .820194$

H $3.0976851 .044823-0.010984$

C $2.951558-0.6783151 .261415$

C $4.881246-1.7657410 .063692$

H $5.076319-0.085179-1.278287$

H $4.779941-1.621654-2.099604$

H 0.3838880 .9412152 .220185

C 1.4439352 .6867581 .540001

C - 0.9946092 .1977431 .168122

H -2.907584 -0.814162 0.592041

C -3.126604 -0.862464 2.738730

C - $1.128258-1.3180744 .004526$

H $0.673691-1.6408112 .864841$

H $2.379561-1.6229931 .287637$

H $2.657545-0.1025302 .154263$

C $4.444631-0.9876861 .299265$

H $5.966412-1.9541180 .092269$

H $4.390220-2.7548790 .067654$

H 1.4903153 .3308880 .644413

H 2.4480612 .2570451 .679939

C 1.0791483 .5365032 .756503

C - 1.3605703 .0349892 .387773

$\mathrm{H}-1.0053882 .8353230 .266292$

$\mathrm{H}-1.7391401 .4052391 .010214$

H -4.198057 -0.649083 2.697126

C -2.493067 -1.036077 3.968710

H -0.624138 -1.470935 4.962559

H $4.682297-1.5512472 .215662$

H $5.013229-0.0415691 .359481$

H 1.8236804 .3383852 .887365

H 1.1384542 .9058513 .661673

C - 0.3236534 .1224052 .643388

$\mathrm{H}-1.4344522 .3712093 .267462$

H -2.3595973.478786 2.249612

H - 0.5736874 .6909313 .553559

H -0.3462004 .8461701 .808460$

$\mathrm{H}-3.063023-0.9584644 .897508$

C $0.4857261 .420196-1.562309$

C $1.5083922 .310913-1.929633$

C -0.727154 $1.443195-2.285462$

C $1.3499143 .216943-2.972186$

H $2.4547432 .304303-1.387561$

C -0.873186 2.375839-3.323905 
C $0.1458743 .255433-3.669357$

H $2.1672973 .892387-3.234079$

$\mathrm{H}-1.8141432 .385063-3.878701$

H $0.0023533 .962716-4.489434$

C - $1.8823760 .538507-2.042240$

C $-1.804266-0.824371-2.357219$

C $-3.1027701 .062047-1.596168$

C $-2.918921-1.650100-2.210092$

H $-0.875929-1.244772-2.749987$

C $-4.2197580 .239256-1.461176$

H -3.172601 2.125444 -1.353949

C $-4.128230-1.119599-1.764629$

$\mathrm{H}-2.821546-2.713517-2.436819$

$\mathrm{H}-5.1645300 .661356-1.110416$

H -5.000399 -1.767396 -1.650373

L2-Pd(Ar) $-\mathrm{Cl}$-trans = trans-2

$E=-2092.10498695$

Cl $0.924446-1.5154673 .127288$

Pd -0.1065130 .0617541 .708437$

H 2.800998 -1.101418 1.546783

C $3.257047-0.9770760 .550286$

P $0.748042-0.023876-0.349245$

C -1.173607 1.6271631 .193830

C $2.5788880 .188397-0.171615$

C $4.755855-0.7302010 .687414$

H $3.074350-1.9209880 .014559$

C $0.2175341 .244712-1.600239$

C -2.5519891.4659781.012280

C -0.6404412 .9208311 .266477$

H $2.9848930 .254463-1.196657$

C 2.8673281 .5181340 .532706

C 5.0436950 .5890031 .393991

H $5.226118-0.717667-0.313787$

H $5.215356-1.5678361 .235590$

H $0.1574642 .173996-1.009490$

C $1.1827701 .470716-2.764204$

C - $1.1858670 .935009-2.123833$

$\mathrm{H}-2.9828260 .4648550 .942560$

C -3.385390 2.5827200 .921869

C - 1.4782904 .0344041 .175536

H 0.4339593 .0684411 .407087

H 2.3971101 .4871491 .531884

H $2.4028752 .357570-0.010926$

C 4.3672341 .7523360 .679344

H 6.1296550 .7619691 .464643

H 4.6652640 .5317212 .429798

H $1.2790520 .540952-3.352132$

H $2.1893241 .728649-2.401080$

C $0.6730442 .593728-3.667129$

C $-1.6961402 .066189-3.008114$

$\mathrm{H}-1.149460-0.000852-2.708882$

$\mathrm{H}-1.8759180 .764273-1.286770$

$\mathrm{H}-4.4609742 .4414730 .785081$

C -2.851499 3.8686630 .997145

$\mathrm{H}-1.0516325 .0387041 .245424$ 
H 4.5425152 .6962181 .220171 H $4.8165651 .879974-0.322657$ H $1.3637912 .729800-4.514988$ H $0.6885913 .540691-3.098247$ C $-0.7428412 .332306-4.166504$ $\mathrm{H}-1.8078182 .977698-2.394389$ $\mathrm{H}-2.7024431 .820316-3.383685$ $\mathrm{H}-1.0955273 .181300-4.774005$ $\mathrm{H}-0.7335861 .452956-4.835882$ H -3.504537 4.7412150.919038 C $0.512282-1.607404-1.252886$ C $1.518872-1.970102-2.163711$ C $-0.664901-2.385959-1.194937$ C $1.384320-3.068813-3.004965$ H $2.433510-1.379598-2.225638$ C - $0.785381-3.483121-2.061830$ C $0.219805-3.828678-2.957181$ H $2.190043-3.325243-3.696275$ H -1.696761 -4.082836 -2.007481 H $0.095058-4.695018-3.610813$ C $-1.816123-2.148517-0.281512$ C $-1.703880-2.3894241 .094220$ C -3.066543 -1.804499-0.810679 C -2.819997 -2.269982 1.922416 H $-0.745200-2.6876951 .523564$ C -4.182242 -1.692614 0.018098 H -3.163403 -1.626257 -1.884520 C -4.060410 -1.923156 1.388143 H -2.708463 -2.457590 2.992728 H -5.150328 $-1.421621-0.409854$ H -4.933021 -1.833834 2.039429

L2-Pd( $\mathrm{Ar})-\mathrm{Cl}\left(\mathrm{HNMe}_{2}\right)=\mathbf{3}$

$E=-2227.19723553$

Pd $0.7195730 .410548-1.075620$ C -1.203295 -2.252433 0.128509 H -2.275200 -2.431846 0.340883 C - $0.904028-2.809647-1.269220$ H $0.161555-2.643533-1.479138$ $\mathrm{H}-1.455688-2.268431-2.049079$ C -1.205664 -4.303019-1.341732 H -2.289328 -4.467409-1.196171 H $-0.968667-4.679453-2.350141$ C $-0.427313-5.077612-0.285435$ H $0.652116-5.004511-0.508279$ $\mathrm{H}-0.685717-6.148517-0.321027$ C $-0.680898-4.5103591 .105726$ $\mathrm{H}-1.737065-4.6825091 .385172$ $\mathrm{H}-0.071040-5.0409911 .854598$ C -2.752959 $0.157289-0.324238$ $\mathrm{H}-3.392395-0.4251830 .367692$ C -3.145609 -0.204719-1.758092 $\mathrm{H}-2.4363830 .282624-2.447596$ H -3.070866 -1.289953 -1.914106 C -4.572498 $0.236291-2.076683$ H -5.282262 -0.353724 -1.467280 
H -4.798940 $0.001852-3.129425$

C -4.788820 1.716864-1.797755

$\mathrm{H}-5.8309952 .002331-2.015210$

H -4.146629 2.310386 -2.471663

C $-4.4311622 .044035-0.354309$

$\mathrm{H}-4.5621983 .119876-0.154978$

$\mathrm{H}-5.1226341 .5135420 .326449$

C -2.993579 1.641961-0.042666

$\mathrm{H}-2.7562871 .8735501 .007464$

H -2.316351 2.226655 -0.685776

$\mathrm{Cl}-0.4835592 .037913-2.442360$

C $1.922388-0.940113-0.234094$

C $2.368774-0.8876261 .091656$

C $2.444236-1.957698-1.050505$

C $3.289755-1.8137201 .586568$

H $1.989255-0.1170131 .761116$

C $3.360915-2.889722-0.560424$

H $2.127352-2.040594-2.094445$

C $3.787127-2.8240960 .765951$

H $3.612860-1.7462732 .629221$

H $3.741714-3.673447-1.221498$

H $4.501629-3.5537551 .154560$

N $2.3969631 .220066-2.169239$

H $1.9659672 .141487-2.287796$

C $3.6652541 .342596-1.461171$

H $3.4985671 .827135-0.491070$

H $4.0824860 .343466-1.276845$

H $4.3937991 .932158-2.047089$

C $2.5364240 .628797-3.495340$

H $2.862219-0.416545-3.402854$

H $1.5626000 .666126-4.001413$

H $3.2844671 .171345-4.101962$

P $-1.023550-0.3889660 .149207$

C - 1.0724840 .0583691 .945848

C -1.997628 -0.6782922 .706480$

C - 0.3175821 .0649072 .595606

C - $2.170447-0.4783634 .069588$

H -2.603006 -1.444595 2.218029

C -0.501864 1.242516 3.980071

C -1.404409 0.4878394 .715442

$\mathrm{H}-2.900062-1.0755634 .620888$

H 0.0662592 .0318704 .476613

H - 1.5221220 .6680835 .786432

C 0.6418172 .0003811 .952829

C 1.8897462 .2391692 .553728

C 0.3118952 .7319070 .806887

C 2.7743753 .1734192 .025217

H 2.1751061 .6702323 .442248

C 1.1941393 .6744370 .278219

H -0.6581932.6013810.333869

C 2.4262313 .9002250 .884795

H 3.7419463 .3347492 .506346

H $0.8976824 .219903-0.620235$

H 3.1164294 .6407890 .473081

C -0.375124 -3.016747 1.166728

H $-0.557422-2.6357132 .181284$ 
H $0.693817-2.8566790 .965205$

$\mathbf{L} 3=\mathrm{PtBu}_{3}$

L3-Pd(Ar) $-\mathrm{NMe}_{2}$

$E=-1307.05489025$

Pd $-0.711530-0.966833-0.002633$

P 1.3446230 .1486370 .021086

C -2.019641 0.5184000 .010160

C -2.681699 0.849658 1.200288

C -2.375867 1.201441-1.159277

C -3.645949 1.858869 1.226612

$\mathrm{H}-2.4492780 .3146032 .125172$

C -3.343698 2.208599-1.132265

H -1.902552 $0.951773-2.111229$

C -3.978671 2.5476260 .061094

$\mathrm{H}-4.1440542 .1034512 .169119$

H -3.603808 2.729278 -2.058312

$\mathrm{H}-4.7345753 .3363200 .081044$

$\mathrm{N}-2.116652-2.333957-0.076150$

C -2.886235 -2.589888 1.110204

$\mathrm{H}-2.258051-2.5221192 .012124$

H -3.743689-1.892161 1.236562

H -3.313430 -3.6135391.085109

C -2.942197 -2.416805-1.251288

H -3.800930 -1.710364-1.232405

H -2.356374 -2.210399-2.159910

H -3.368587 -3.435427 -1.357923

C 1.4469331 .9369370 .691592

C 0.5618242 .0403821 .941058

C 2.8579912 .4188511 .035819

C $0.8274962 .887937-0.339701$

H -0.467369 1.7284031 .725328

H 0.9322751 .4567062 .789568

H 0.5331723 .0966522 .256250

H 3.5511522 .3661830 .186912

H 2.8008583 .4748541 .348892

H 3.2976391 .8616271 .873762

H 0.7398273 .8845070 .122929

H $1.4407623 .004439-1.242366$

H - $0.1848432 .570577-0.628137$

C $2.0112170 .127085-1.776016$

C $3.2638440 .966764-2.029303$

C $2.293104-1.320453-2.194530$

C $0.8746740 .601341-2.692841$

H $3.0843952 .040782-1.891137$

H $4.1062470 .674489-1.388839$

H $3.5814840 .825644-3.076176$

H $1.424115-1.971348-2.011964$

H $2.491467-1.333723-3.278872$

H $3.171551-1.753681-1.700131$

H $1.2197430 .551187-3.739094$

H - $0.000358-0.057715-2.587534$

H $0.5493871 .628243-2.497266$

C $2.469536-0.9741661 .097761$

C $3.973685-0.8368220 .858804$ 
$\begin{array}{llll}\text { C } 2.172829 & -0.702823 & 2.576519 \\ \text { C } 2.043533 & -2.430785 & 0.852137 \\ \text { H } 4.268759 & -1.143621 & -0.153038 \\ \text { H } 4.336739 & 0.185424 & 1.024752 \\ \text { H } 4.509534 & -1.494914 & 1.563685 \\ \text { H } 1.093465 & -0.746257 & 2.786922 \\ \text { H } 2.661856 & -1.485852 & 3.179125 \\ \text { H } 2.561916 & 0.262805 & 2.922682 \\ \text { H } 2.609138 & -3.085051 & 1.536744 \\ \text { H } 0.971949 & -2.580622 & 1.072000 \\ \text { H } 2.231808 & -2.781608 & -0.167412\end{array}$

TS (reductive elimination)

$E=-1307.03827756$

Pd $0.616647-1.034112-0.054972$

$\mathrm{P}-1.3988390 .090881-0.012211$

C $2.2532940 .158451-0.014399$

C $2.7103250 .741262-1.208641$

C 2.6203250 .7652871 .198633

C $3.4493961 .923411-1.189256$

H $2.4750170 .279448-2.169609$

C 3.3602091 .9469811 .209744

H 2.3099910 .3275732 .148853

C 3.7741012 .5416300 .017752

H $3.7735412 .364388-2.136100$

H 3.6130472 .4074982 .168978

H 4.3523833 .4680310 .030137

N $2.421182-1.908225-0.017885$

C $3.180863-2.215917-1.193893$

H $2.599419-2.011813-2.103722$

H $4.133782-1.646445-1.257638$

H $3.435734-3.293387-1.208970$

C $3.121595-2.2089491 .196785$

H $4.072989-1.6431041 .301222$

H $2.497931-1.9918642 .075113$

H $3.369045-3.2875511 .235665$

C -1.349450 $1.791296-0.890140$

C -0.580239 1.627571-2.208191

C -2.710865 2.424052 -1.182938

C $-0.5132382 .749742-0.033241$

H $0.3869101 .137187-2.038670$

$\mathrm{H}-1.1312911 .063604-2.967312$

$\mathrm{H}-0.3808192 .630042-2.621720$

$\mathrm{H}-3.3186022 .576875-0.282346$

H -2.548812 $3.413894-1.641948$

$\mathrm{H}-3.2997511 .833633-1.898027$

H $-0.3313173 .666418-0.617435$

$\mathrm{H}-1.0212163 .0516430 .891645$

H 0.4679772 .3207680 .219083

C -1.9188180.3585401.814001

C -3.041582 1.3715402 .041870

C - $2.329343-0.9855452 .425411$

C -0.6680090 .7906592 .591892$

$\mathrm{H}-2.7440622 .3926191 .769524$

$\mathrm{H}-3.9581751 .1225471 .491084$

$\mathrm{H}-3.2960581 .3865583 .115158$ 
H -1.558319 -1.753439 2.259969

$\mathrm{H}-2.435113-0.8520613 .514694$

H -3.290029 -1.360768 2.051126

H -0.938065 0.9172973 .653934

H 0.1085860 .0154152 .521045

H -0.228058 1.7296182 .241453

C - $2.728244-1.000436-0.860498$

C $-4.182761-0.657601-0.535953$

C - $2.531557-0.931976-2.378384$

C -2.440038 -2.458843 -0.471599

$\mathrm{H}-4.426157-0.8094650 .523593$

$\mathrm{H}-4.4398270 .376647-0.799381$

H -4.846699 -1.319976-1.117022

$\mathrm{H}-1.483845-1.118386-2.659077$

$\mathrm{H}-3.148023-1.717400-2.846060$

$\mathrm{H}-2.8479370 .026975-2.807673$

H -3.114873 -3.119601-1.041561

$\mathrm{H}-1.403480-2.737607-0.726455$

H -2.593025 -2.669225 0.591796

L3-Pd-( $\left.\mathrm{ArNMe}_{2}\right)$

$E=-1307.09172647$

Pd $0.480654-0.8701450 .050360$

$\mathrm{P}-1.5221820 .083876-0.023267$

C $3.158839-0.2294920 .043985$

C $3.7855870 .268969-1.105831$

C 3.0598070 .6155591 .165912

C $4.2575491 .582101-1.144047$

H $3.893867-0.351795-1.993360$

C 3.5416831 .9158621 .120035

H 2.5581880 .2649842 .068040

C $4.1402672 .416085-0.038931$

H $4.7281751 .948158-2.059838$

H 3.4338792 .5530322 .001070

H $4.5098543 .442842-0.074616$

N 2.605645 -1.551100 0.094785

C $2.849471-2.375446-1.082377$

H $2.449069-1.887470-1.979091$

H $3.929318-2.581873-1.225820$

H $2.318845-3.327162-0.955595$

C $2.925450-2.2979381 .311603$

H $4.009675-2.5165881 .373234$

H $2.622540-1.7426492 .204773$

H $2.365418-3.2416841 .304069$

C - $1.2901231 .996199-0.006726$

C - $0.0980582 .337940-0.914546$

C $-2.5040772 .827152-0.424783$

C -0.8570772.4168851.401601

H $0.7944321 .765633-0.616094$

H -0.284989 2.139625 -1.975098

H $0.1261913 .413680-0.811937$

H -3.390657 2.6269770.191163

$\mathrm{H}-2.2625863 .898377-0.314894$

H -2.777223 2.668876 -1.476733

$\mathrm{H}-0.5209263 .4666431 .366165$

H -1.667875 2.360177 2.139277 
$\mathrm{H}-0.0108981 .8051351 .750461$

C -2.612903 -0.391305 1.486935

C -3.814897 0.5107081 .769321

C -3.101450 -1.830254 1.291932

C -1.702178 -0.4319902 .723216$

$\mathrm{H}-3.5163901 .5302982 .047684$

$\mathrm{H}-4.5043410 .5784420 .917664$

$\mathrm{H}-4.3836450 .0990682 .620868$

H -2.264829 -2.499710 1.039298

$\mathrm{H}-3.544590-2.1834232 .238142$

H -3.874632 -1.921492 0.518345

$\mathrm{H}-2.267807-0.8601873 .568632$

H -0.821706 -1.064267 2.525900

H -1.342922 0.5556013 .031687

C $-2.488548-0.379973-1.619086$

C $-3.980979-0.047088-1.633690$

C - $1.8095780 .311280-2.805997$

C $-2.297093-1.885323-1.857021$

$\mathrm{H}-4.544409-0.617387-0.882949$

$\mathrm{H}-4.1801251 .020168-1.470863$

H $-4.400596-0.313125-2.619170$

$\mathrm{H}-0.7235150 .130341-2.797987$

H -2.217594 -0.110193 -3.739943

$\mathrm{H}-1.9869781 .393921-2.833351$

$\mathrm{H}-2.691932-2.141251-2.855289$

$\mathrm{H}-1.226813-2.143375-1.818618$

H -2.818467 -2.511721 -1.125322

L3-Pd(ArCl)

$E=-1632.69884924$

Pd $-0.936140-0.3184220 .050589$

P $1.340042-0.007691-0.011164$

Cl -3.379706 -2.196287 0.045949

C -3.047301 -0.4716850 .010980$

C -2.834847 0.2324311 .226380

C -3.270794 0.248510-1.191088

C -2.935066 1.6387651 .224769

$\mathrm{H}-2.778655-0.3160232 .168611$

C -3.357700 $1.636568-1.153459$

$\mathrm{H}-3.434927-0.303713-2.117816$

C -3.215043 2.333362 0.054682

$\mathrm{H}-2.8107452 .1740112 .169107$

$\mathrm{H}-3.5480282 .181254-2.081109$

$\mathrm{H}-3.3073083 .4210930 .072266$

C $1.7299181 .539940-1.076731$

C $0.6909632 .620114-0.736399$

C $3.1382822 .116594-0.928196$

C $1.4842361 .187360-2.547529$

H - $0.3322012 .222440-0.828287$

H 0.8004673 .0286890 .273510

H $0.8076573 .456231-1.446643$

H $3.9232011 .385241-1.161539$

H $3.2573702 .961733-1.627372$

H 3.3254752 .5082330 .080610

H $1.5162372 .116856-3.139598$

H $2.2425850 .511973-2.963583$ 
H $0.4893600 .737005-2.687213$

C $2.221067-1.522715-0.786382$

C $3.655700-1.296148-1.264158$

C $2.207274-2.6661180 .233666$

C $1.354792-2.008504-1.958939$

H $3.713453-0.574371-2.089770$

H $4.318869-0.948920-0.460833$

H 4.065543 -2.248752 -1.640879

H $1.195019-2.8346960 .631718$

H $2.525148-3.591713-0.274068$

H $2.895959-2.5038551 .072515$

H $1.758096-2.967539-2.325698$

H $0.315266-2.173103-1.631927$

H $1.334766-1.314070-2.805254$

C 2.0425760 .2731301 .751142

C 1.5995511 .6594072 .230490

C $1.355381-0.7272792 .692581$

C 3.5603190 .1536661 .892918

H 2.1263602 .4784611 .724622

H 0.5154541 .7977872 .097406

H 1.8214881 .7450233 .307050

H $1.657351-1.7659372 .522483$

H $1.616771-0.4721153 .733404$

H $0.260972-0.6709402 .581908$

H 3.8445120 .3838102 .933903

H $3.922003-0.8612041 .680146$

H 4.1034420.852396 1.243191

TS (oxidative addition)

$E=-1632.68136790$

Pd $-0.659699-1.1661160 .040191$

P 1.2629360 .1047590 .006401

Cl $-2.737647-2.311582-0.015063$

C -2.634544 -0.354051-0.001996

C -2.992792 0.2541031 .213296

C - $2.9213340 .281927-1.221887$

C -3.503952 1.5484291 .200110

$\mathrm{H}-2.849329-0.2843612 .151373$

C -3.430794 $1.576250-1.208400$

$\mathrm{H}-2.722395-0.233300-2.162553$

C -3.715897 2.223154-0.003462

$\mathrm{H}-3.7458462 .0322052 .149991$

H -3.615560 2.082505 -2.159458

H $-4.1225803 .236292-0.004083$

C $1.7070680 .570243-1.800850$

C $0.4040330 .941701-2.522578$

C $2.7245281 .700354-1.964389$

C $2.226753-0.678592-2.520797$

H - $0.3081140 .105019-2.480223$

$\mathrm{H}-0.0974271 .822368-2.108746$

H $0.6346151 .150234-3.581016$

H $3.6761501 .492937-1.457715$

H $2.9447841 .830313-3.037499$

H $2.3432712 .662510-1.597675$

H $2.288526-0.457207-3.599038$

H $3.229395-0.982694-2.195090$ 
H 1.537932 -1.527850 -2.394854

C 1.0592081 .7175271 .020606

C 0.3237071 .3751372 .323771

C 2.3553782 .4572701 .354107

C 0.1234012 .6522410 .246160

H - 0.5974220 .8137132 .115041

H 0.9297960 .7959243 .027565

H 0.0433542 .3167382 .824805

H 2.9333852 .7324430 .462993

H 2.104964 3.3909651 .885498

H 3.0077391 .8762942 .019925

H -0.1498613.493618 0.903716

H $0.5898063 .080554-0.650206$

H -0.809617 2.146067 -0.042579

C $2.714697-0.9078810 .744371$

C $2.580096-2.3537460 .243119$

C $4.117661-0.3843720 .432760$

C $2.536723-0.9795632 .264329$

H $1.579412-2.7558290 .471357$

H $2.747288-2.462190-0.833353$

H $3.327232-2.9790520 .760399$

H 4.2691880 .6479940 .773921

H $4.859085-1.0146110 .952555$

H $4.355681-0.428369-0.638123$

H 3.241672 -1.727336 2.663378

H $2.753949-0.0284742 .766697$

H $1.519407-1.3013702 .534654$

L3-Pd(Ar)-Cl

$E=-1632.72611518$

Pd $0.6652371 .155501-0.043834$

$\mathrm{P}-1.151678-0.2543490 .022312$

Cl $2.1222632 .926388-0.133449$

C $2.115036-0.158729-0.049678$

C $2.813108-0.3498911 .146558$

C $2.528318-0.821418-1.205049$

C $3.883875-1.2437161 .192999$

H 2.5333860 .2049642 .044328

C $3.603146-1.713126-1.149233$

H $2.031959-0.645909-2.158890$

C $4.276590-1.9369930 .049095$

H $4.420301-1.3878602 .134605$

H $3.916781-2.229268-2.060603$

H $5.116364-2.6346120 .087953$

C - $1.052509-1.9603780 .867931$

C - $2.413136-2.5314081 .275766$

C $-0.346331-2.943861-0.073161$

C - $0.165076-1.8490712 .114065$

$\mathrm{H}-2.901271-1.9287222 .053217$

H -3.109033 -2.637638 0.434754

H -2.254904 -3.536153 1.701301

H $0.631349-2.562493-0.401980$

H - $0.165229-3.8774670 .483518$

H - $0.946668-3.204535-0.954057$

H - $0.039546-2.8603392 .534547$

H $0.831242-1.4669771 .862650$ 
H $-0.592041-1.2191162 .900368$

C - $1.831858-0.464647-1.754965$

C -3.000337 -1.443319-1.884581

C - $2.2602420 .898910-2.309824$

C - $0.672567-0.916308-2.652502$

$\mathrm{H}-2.709033-2.477200-1.658551$

$\mathrm{H}-3.851901-1.177298-1.244751$

H -3.356325 -1.430580 -2.928129

$\mathrm{H}-1.4516101 .641466-2.229670$

$\mathrm{H}-2.4821430 .775476-3.382259$

$\mathrm{H}-3.1647631 .301334-1.837629$

H -1.056636 -1.057554 -3.676223

H $0.103782-0.139211-2.687933$

H -0.203833 -1.854529-2.338814

C -2.3428850.882296 1.002400

C -2.015220 0.7942632.496716

C -2.015603 2.3286690.588639

C -3.834153 0.6105720 .805704

$\mathrm{H}-2.324289-0.1568952 .948001$

$\mathrm{H}-0.9428370 .9497052 .688067$

$\mathrm{H}-2.5642811 .5952753 .018007$

$\mathrm{H}-2.2186782 .552630-0.462692$

H -2.623979 3.019281 1.196188

H -0.9623182.5967360.808740

$\mathrm{H}-4.4084211 .3058281 .440772$

H -4.163914 $0.773293-0.228173$

H -4.116657 -0.409113 1.098357

cis-trans isomerization

$E=-1632.71838131$

Pd -0.568029 1.233479-0.006851

P $0.954086-0.4379470 .009035$

Cl $-0.5423113 .565332-0.047046$

C -2.097692 $0.041502-0.000339$

C - $2.677549-0.398746-1.193005$

C -2.767161 -0.154705 1.213092

C -3.911901-1.051284-1.166487

H -2.182258 -0.222337-2.150654

C -4.002567 -0.804496 1.228507

$\mathrm{H}-2.3318100 .2029582 .149672$

C -4.572769-1.265334 0.042087

H -4.360156 -1.391076 -2.103981

H -4.521033 -0.9510952 .179728$

H -5.535928 -1.780372 0.058470

C $0.664610-2.1310370 .858279$

C $1.803752-3.1269120 .609731$

C $0.510862-1.8978542 .364950$

C - $0.643466-2.7876200 .393250$

H $1.833870-3.467296-0.433702$

H $2.793813-2.7413540 .876145$

H $1.619656-4.0188891 .230837$

H $-0.250826-1.1348192 .580805$

H $0.174051-2.8402542 .826070$

H $1.449327-1.6121342 .855899$

H - $0.704240-3.7750790 .879950$

$\mathrm{H}-1.523770-2.2122080 .695740$ 
H $-0.700043-2.951756-0.686190$

C 2.3594660 .4960740 .946914

C $3.553246-0.3766541 .341295$

C 2.8638781 .6629380 .086050

C 1.7677881 .1395752 .213297

H $3.295605-1.1289692 .098070$

H $4.016737-0.8857520 .486656$

H 4.3208110 .2763311 .788063

H $2.0498432 .339710-0.216915$

H 3.5577692 .2560830 .703334

H $3.4193231 .340746-0.803223$

H 2.5849711 .6561912 .743192

H 1.0080741 .8962461 .964075

H 1.3251240 .4211262 .910591

C $1.495629-0.731711-1.804871$

C $0.596608-1.792251-2.448562$

C $1.2654750 .567325-2.590938$

C $2.953938-1.171560-1.961346$

H $0.798604-2.805829-2.079993$

H -0.470097 -1.572608 -2.307157

H $0.794922-1.794880-3.532595$

H $1.8427451 .418396-2.215251$

H $1.5629210 .395900-3.638798$

H $0.2050380 .860172-2.579923$

H $3.144237-1.364820-3.029991$

H $3.666237-0.400319-1.643587$

H $3.180921-2.096965-1.417353$

L3-Pd(Ar)-Cl -trans

$E=-1632.71902157$

Cl -0.5089723 .4062850 .000503$

Pd $0.5135121 .291820-0.013384$

P $-0.878105-0.4938800 .004626$

C $2.1479690 .236508-0.016114$

C $2.8267770 .059551-1.229198$

C $2.813038-0.0298471 .186806$

H $2.3269940 .272810-2.178264$

C $4.151973-0.380043-1.234846$

C $4.137305-0.4706351 .174175$

H 2.3047550 .1138092 .144066

H $4.674034-0.509090-2.186745$

C $4.806947-0.656098-0.035002$

H $4.648381-0.6717132 .119556$

H $5.840910-1.008735-0.041968$

C $-0.300724-2.230666-0.588474$

C $1.026510-2.6674910 .050086$

C -1.328504 -3.328864 -0.282421

C $-0.039336-2.172909-2.097724$

H $1.875103-2.091010-0.328039$

H $1.038900-2.6120561 .141873$

H $1.188866-3.721711-0.228808$

$\mathrm{H}-2.341575-3.106636-0.631437$

$\mathrm{H}-1.000521-4.250469-0.790396$

$\mathrm{H}-1.373225-3.5574310 .790755$

H $0.431508-3.122374-2.399856$

H -0.949321 -2.054570 -2.697575 
H $0.660820-1.365377-2.352434$

C - $1.532713-0.6375051 .796991$

C -1.586957 0.7710362 .404296

C $-2.912374-1.2948671 .903144$

C - $0.543981-1.4405912 .647380$

$\mathrm{H}-0.5803191 .2058182 .488064$

$\mathrm{H}-2.1937751 .4802711 .832747$

$\mathrm{H}-2.0125650 .6925893 .418431$

$\mathrm{H}-2.935957-2.3101211 .489781$

H -3.174928 -1.370376 2.971194

$\mathrm{H}-3.701574-0.7064601 .420562$

$\mathrm{H}-0.835805-1.3308383 .704343$

$\mathrm{H}-0.552188-2.5137762 .417976$

H $0.484058-1.0656442 .547871$

C -2.307262 $0.088224-1.156268$

C -3.131026 1.201866 -0.499031

C - $1.6684590 .710536-2.408529$

C $-3.277042-1.023309-1.571280$

$\mathrm{H}-3.7339320 .8542110 .348662$

H -2.505387 $2.050958-0.189055$

$\mathrm{H}-3.8336821 .578899-1.259786$

H -0.992831 $0.034188-2.944187$

$\mathrm{H}-2.4778230 .992252-3.102051$

$\mathrm{H}-1.1181171 .626457-2.147570$

$\mathrm{H}-4.068156-0.562681-2.184687$

$\mathrm{H}-2.811879-1.803780-2.185427$

H -3.771086 -1.499189-0.713770

L3-Pd(Ar)-Cl(HNMe $\left.{ }_{2}\right)$

$E=-1767.80524962$

Pd $-0.679954-0.8183940 .018923$

P 1.3930480 .3067250 .002548

Cl $0.212495-3.0960090 .171956$

C - $1.8681020 .769017-0.031852$

C -2.357200 1.3306361 .158375

C -2.444860 $1.202064-1.235000$

C -3.365272 2.296152 1.146079

$\mathrm{H}-1.9489231 .0099362 .120746$

C -3.453848 2.168713-1.252505

$\mathrm{H}-2.1079150 .778155-2.186051$

C -3.916601 2.724354-0.061263

$\mathrm{H}-3.7217042 .7170362 .090506$

H -3.880007 2.487868 -2.207882

$\mathrm{H}-4.7035563 .482204-0.072903$

$\mathrm{N}-2.503609-1.9960930 .018868$

$\mathrm{H}-2.007397-2.8552720 .285376$

C -3.540174 -1.698884 0.996730

H -3.093223 -1.613332 1.996308

$\mathrm{H}-4.022441-0.7421320 .755409$

H -4.309365 -2.492069 1.012700

C -3.032282 -2.175822 -1.327180

$\mathrm{H}-3.428156-1.220960-1.700586$

$\mathrm{H}-2.224149-2.513061-1.990098$

H -3.845625 -2.924171-1.343516

C $1.4084332 .186581-0.452725$

C 0.5842332 .9687460 .576519 
C $2.7748592 .893258-0.539130$

C $0.7265882 .334043-1.816392$

H -0.396480 2.5223120 .766965

H 1.1193043 .0876851 .528599

H 0.4120573 .9809870 .175946

H $3.2967372 .706934-1.481061$

H $2.5836553 .977859-0.494580$

H 3.4579752 .6544810 .281046

H $0.6507923 .407909-2.055009$

H $1.2973461 .860326-2.625831$

H -0.288556 $1.923569-1.810775$

C $2.497269-0.575647-1.319541$

C $3.5986190 .291310-1.931855$

C $3.194188-1.825570-0.777313$

C $1.536801-1.021538-2.433320$

H $3.2125861 .110501-2.549506$

H $4.2831370 .703698-1.177908$

H $4.196324-0.354086-2.596130$

H $2.504963-2.506412-0.266281$

H $3.614269-2.366169$-1.641878

H $4.034491-1.569761-0.117977$

H $2.132230-1.426195-3.269173$

H $0.868859-1.815544-2.071327$

H $0.922157-0.199606-2.825378$

C 2.0521340 .1397921 .804351

C 3.2359391 .0476052 .135365

C 0.8642200 .4744732 .719315

C $2.450571-1.2994742 .150375$

H 4.0846180 .8888441 .452916

H 2.9743122 .1135462 .131787

H 3.5861890 .8039883 .152168

H $0.062927-0.2695352 .590242$

H 1.2072030 .4293673 .766279

H 0.4371391 .4681602 .548785

H $2.495133-1.3722503 .249662$

H $1.722269-2.0392811 .789893$

H $3.442768-1.5643281 .770331$ 


\subsubsection{Energies and Coordinates Related to Scheme 4}

Table S17. SCF energies and enthalpy/free energy corrections for Scheme 4.

\begin{tabular}{|c|c|c|c|c|c|c|}
\hline $\begin{array}{c}\text { L1 = } \\
\text { YPhos }\end{array}$ & Corr(H) & Corr(G) & $E(S C F)$ & $\mathrm{E}(\mathrm{PCM})$ & $\underset{H}{\Delta G(P C M) / E}$ & $\begin{array}{l}\Delta \mathrm{G}(\mathrm{PCM} \\
\mathrm{T} / \mathrm{kJ} / \mathrm{mol}\end{array}$ \\
\hline trans-3 & 1.09748 & 0.965979 & -2889.288826 & -2889.305039 & 0.002414 & 6.338 \\
\hline cis-2 & 0.995407 & 0.872005 & -2754.212956 & -2754.227583 & 0 & 0 \\
\hline $\begin{array}{l}\text { cis-trans- } \\
\text { isomerizati } \\
\text { on }\end{array}$ & 0.994954 & 0.873402 & -2754.207516 & -2754.220982 & 0.007998 & 21.00 \\
\hline trans-2 & 0.996002 & 0.872357 & -2754.208345 & -2754.221645 & 0.006290 & 16.52 \\
\hline 3 & 1.097191 & 0.963834 & -2889.30644 & -2889.314253 & -0.008944 & -23.48 \\
\hline cis-2-distal & 0.995497 & 0.871766 & -2754.197246 & -2754.21985 & 0.007494 & 19.68 \\
\hline $\begin{array}{l}\text { cis-trans- } \\
\text { isomerizati } \\
\text { on-distal }\end{array}$ & 0.994833 & 0.872536 & -2754.19156 & -2754.213065 & 0.015049 & 39.511 \\
\hline $\begin{array}{c}\text { trans-2- } \\
\text { distal }\end{array}$ & 0.996253 & 0.873264 & -2754.192414 & -2754.212036 & 0.016806 & 44.125 \\
\hline 3-distal & 1.096963 & 0.962557 & -2889.293933 & -2889.305779 & -0.001747 & -4.588 \\
\hline \multicolumn{7}{|l|}{$\begin{array}{c}\text { L2 }= \\
\text { CyJohnPho } \\
\text { S }\end{array}$} \\
\hline trans-3 & 0.725929 & 0.622068 & -2227.170735 & -2227.186238 & 0.008248 & 21.66 \\
\hline cis-2 & 0.623903 & 0.527819 & -2092.097159 & -2092.114341 & 0 & \\
\hline $\begin{array}{l}\text { cis-trans- } \\
\text { isomerizati } \\
\text { on }\end{array}$ & 0.623099 & 0.527871 & -2092.086411 & -2092.102107 & 0.012286 & 32.256 \\
\hline trans-2 & 0.624368 & 0.527659 & -2092.08837 & -2092.104987 & 0.009194 & 24.14 \\
\hline 3 & 0.726197 & 0.621514 & -2227.186965 & -2227.197236 & -0.003303 & -8.672 \\
\hline cis-2-distal & 0.624061 & 0.526221 & -2092.084268 & -2092.105109 & 0.007634 & 20.04 \\
\hline $\begin{array}{l}\text { cis-trans- } \\
\text { isomerizati } \\
\text { on-distal }\end{array}$ & 0.623061 & 0.527792 & -2092.081605 & -2092.099103 & 0.015211 & 39.937 \\
\hline $\begin{array}{l}\text { trans-2- } \\
\text { distal }\end{array}$ & 0.624267 & 0.526123 & -2092.080486 & -2092.10004 & 0.012605 & 33.093 \\
\hline 3-distal & 0.725815 & 0.618113 & -2227.187041 & -2227.198023 & -0.007491 & -19.67 \\
\hline
\end{tabular}

L1 = YPhos

trans-3

$E=-2889.30503925$

Pd $1.575453-0.5994911 .257487$

C 2.8710930 .8964761 .128832

N $0.567732-2.5978001 .615435$

P $0.865328-0.188597-0.938911$

Cl $2.338581-0.7865113 .495558$

$\mathrm{P}-2.1788080 .206595-0.214382$

C $-0.8584280 .047783-1.301820$

C - -1.6700710 .4699261 .538726$

C -3.321799 $1.563905-0.778595$ 
C $-3.340053-1.263123-0.187465$

C $1.412546-1.599239-2.041888$

C $1.8081471 .234105-1.743570$

C $1.655051-3.5744891 .579093$

C $-0.183557-2.7340182 .858458$

$\mathrm{H}-0.060014-2.7647610 .831617$

C -1.208375 $0.171897-2.777714$

$\mathrm{H}-0.7411801 .048834-3.259256$

$\mathrm{H}-2.2896400 .277650-2.951222$

H -0.901809 -0.710222 -3.368949

C -2.590217 -2.515664 0.258049

C - $4.018990-1.518369-1.536994$

$\mathrm{H}-4.123228-1.0334940 .556689$

C -4.923597-2.746730-1.488963

H -4.609479 $-0.645482-1.854797$

H -3.241989-1.674085 -2.304724

C $-4.161752-3.988633-1.045956$

H $-5.754426-2.557664-0.785283$

H $-5.384441-2.906846-2.476970$

C -3.480228 -3.7529300 .294631$

H -3.396511 -4.234625 -1.804370

H -4.836598 $-4.857705-0.990673$

$\mathrm{H}-4.248194-3.6253401 .078794$

$\mathrm{H}-2.882804-4.6316960 .587493$

$\mathrm{H}-1.759190-2.675547-0.448721$

H -2.143445 -2.357914 1.247742

C -4.504730 1.8498030 .150441

C -2.609659 2.855594-1.182086

H -3.744279 1.144537-1.708967

C $-3.5885653 .833679-1.823525$

$\mathrm{H}-1.7874542 .625742-1.871425$

$\mathrm{H}-2.1465013 .325355-0.300907$

C $-4.7761934 .121769-0.912840$

$\mathrm{H}-3.0653984 .767730-2.083663$

H -3.954305 $3.407703-2.775754$

C -5.476461 $2.833986-0.496174$

H -5.487558 4.802202 -1.407756

H -4.420336 $4.648642-0.008935$

H -5.930465 2.360251 -1.385584

H -6.305344 3.0503190.196977

H -4.133927 2.2826041 .094762

$\mathrm{H}-5.0347340 .9220100 .416499$

C - 1.1476991 .8890501 .784989

C -2.715645 0.0888022 .595712

$\mathrm{H}-0.791964-0.2002831 .642910$

C - 0.5923132 .0314023 .197357

$\mathrm{H}-1.9684142 .6136531 .650074$

H -0.378313 2.130994 1.038923

C -1.6474321.6891584.238679

H 0.2744441 .3576993 .320541

H -0.220849 3.0582533 .344720

C -2.172188 0.279125 4.011640

$\mathrm{H}-2.4829072 .4110804 .175928$

$\mathrm{H}-1.2291131 .7787275 .253399$

$\mathrm{H}-2.9613900 .0305564 .739591$

$\mathrm{H}-1.346017-0.4299434 .186358$ 
$\mathrm{H}-3.6152720 .7136842 .473617$

$\mathrm{H}-3.048011-0.9524852 .470546$

C $0.544517-2.848278-1.943640$

C $2.889486-1.928784-1.824477$

H $1.299908-1.223725-3.073705$

C $1.022326-3.931870-2.905483$

H $0.580363-3.251882-0.918632$

$\mathrm{H}-0.506090-2.586469-2.142106$

C $2.491718-4.270303-2.678506$

H $0.396899-4.834021-2.802279$

H 0.886757 -3.576557 -3.943124

C $3.366669-3.025329-2.771632$

H $2.827275-5.032678-3.400047$

H $2.608205-4.721996-1.676255$

H $4.418619-3.276154-2.559416$

H $3.342280-2.642577-3.808298$

H $3.509535-1.026948-1.943888$

H $3.034156-2.244915-0.776400$

C $2.1315921 .164508-3.240399$

C $1.1465152 .566559-1.406012$

H $2.7740511 .213361-1.215153$

C $3.0138132 .344527-3.645931$

H $2.6550490 .230593-3.491709$

H $1.2092451 .177420-3.844558$

C $2.3686523 .681161-3.302871$

H $3.2413052 .288859-4.723417$

H $3.9798672 .263435-3.115883$

C $2.0117343 .749106-1.823806$

H $1.4510103 .805400-3.907404$

H $3.0373464 .512788-3.579207$

H $1.4922824 .694421-1.594263$

H $2.9342653 .742944-1.217709$

H $0.1697362 .622016-1.912874$

H $0.9425482 .619540-0.327925$

C 2.6352622 .2019581 .567467

C 4.1553710 .5814370 .659378

C 3.6350023 .1750841 .498629

H 1.6658182 .4730361 .985546

C 4.8974062 .8593611 .000299

H 3.4214224 .1904321 .844573

C 5.1559651 .5515800 .590648

H 6.1476781 .2787450 .218907

H $4.385958-0.4391440 .343098$

H $1.284241-4.6059491 .728773$

H $2.366843-3.3237592 .378023$

$\mathrm{H}-1.033835-2.0436502 .868404$

H $0.477617-2.4600233 .691784$

H $2.183538-3.5215100 .619494$

H -0.559447 -3.764828 2.996236

H 5.6784853 .6216640 .946572

cis-2-distal

$E=-2754.21985029$

Pd 2.302494 0.257258 1.733547

P -2.239421 -0.0280660 .248848$

P $0.9473790 .189661-0.103113$ 
C -0.6121050 .2034580 .745433$

C -0.421534 0.4990802 .233397

H -0.162889 1.5492192 .457745

H $0.359918-0.1476002 .701170$

$\mathrm{H}-1.3127880 .2731872 .841402$

C $-3.097419-1.1834721 .422861$

$\mathrm{H}-3.133508-0.5851172 .350459$

C $-4.547105-1.5204271 .065535$

H -4.573622 -2.117396 0.138095

H $-5.127610-0.6062100 .864783$

C -5.206557 -2.315555 2.190597

H -5.294662 -1.667707 3.081510

$\mathrm{H}-6.234848-2.5881671 .903305$

C -4.402701-3.559434 2.551763

$\mathrm{H}-4.411932-4.2580861 .695264$

H -4.880795 -4.091814 3.389399

C -2.958886 -3.209432 2.894945

$\mathrm{H}-2.382508-4.1212253 .117727$

H -2.937846 -2.597883 3.815083

C $-2.288830-2.4368671 .763522$

$\mathrm{H}-1.259990-2.1549242 .032181$

H -2.211580 -3.089833 0.880800

C -2.364573 -0.651367-1.488226

$\mathrm{H}-1.509507-0.155127-1.978818$

C -3.629005 -0.248331-2.257256

$\mathrm{H}-3.8002070 .835259-2.209033$

H -4.510438 -0.727463-1.797492

C -3.522685 -0.672010 -3.720561

H $-4.442458-0.387253-4.255972$

$\mathrm{H}-2.700234-0.105770-4.194057$

C -3.257609 -2.164562 -3.869323

$\mathrm{H}-4.138156-2.726927-3.509598$

H -3.134185 -2.428657 -4.931585

C -2.034667 -2.587138 -3.066204

$\mathrm{H}-1.128007-2.128512-3.501248$

$\mathrm{H}-1.885997-3.677078-3.126981$

C -2.158070 -2.166563-1.606128

H -3.023297 -2.684403-1.160870

H -1.276570 -2.487692 -1.038159

C -3.337305 1.4859520 .325354

$\mathrm{H}-4.3054751 .190349-0.117195$

C -3.599877 1.967001 1.757511

$\mathrm{H}-4.0831841 .1797892 .355695$

H -2.6381012.193023 2.247998

C -4.476339 3.216526 1.775301

$\mathrm{H}-4.6318393 .5435772 .815782$

$\mathrm{H}-5.4752352 .9624691 .376400$

C -3.873461 4.341157 0.944482

$\mathrm{H}-4.5361435 .2211850 .947073$

$\mathrm{H}-2.9232274 .6643611 .406080$

C -3.605331 $3.876860-0.480367$

$\mathrm{H}-3.1149874 .673544-1.063155$

H -4.566052 $3.668419-0.985416$

C -2.740182 2.622035-0.503676

$\mathrm{H}-1.7414402 .850263-0.098125$

$\mathrm{H}-2.5764322 .305278-1.544857$ 
C $1.156433-1.290655-1.200081$

H $0.258124-1.357599-1.836204$

C $2.369476-1.198849-2.131474$

H $3.280993-1.034364-1.536938$

H $2.276587-0.335939-2.807979$

C $2.525378-2.474989-2.954071$

H $1.661861-2.580276-3.637746$

H $3.419848-2.394045-3.592350$

C $2.614395-3.710601-2.067187$

H $3.528883-3.643919-1.451460$

H $2.712658-4.620673-2.681050$

C $1.401806-3.811519-1.149712$

H $0.496112-3.980308-1.761902$

H $1.491245-4.683791-0.482026$

C $1.232883-2.542680-0.322883$

H $0.342873-2.6025830 .323684$

H $2.090850-2.4292960 .360727$

C $1.2354701 .643427-1.214794$

H $2.2751511 .491564-1.556986$

C $1.2033962 .930632-0.390289$

H 0.2044553 .0393950 .066465

H 1.9072082 .8464190 .454529

C $1.5156934 .154984-1.242305$

H $2.5641984 .096729-1.586562$

H $1.4412845 .069407-0.631922$

C $0.5965784 .244394-2.455507$

H $0.8583465 .117280-3.075087$

$\mathrm{H}-0.4422964 .407181-2.113214$

C $0.6549472 .967375-3.286267$

$\mathrm{H}-0.0367913 .029964-4.142626$

H $1.6683332 .856763-3.712245$

C $0.3325721 .737360-2.441643$

H $0.4144450 .825599-3.055699$

H $-0.7146061 .802279-2.106822$

Cl 3.3798530 .3495963 .779647

C 3.9398110 .0586880 .694409

C $4.613750-1.1673850 .730634$

C $4.4676811 .106757-0.064133$

C $5.766642-1.356543-0.032549$

H $4.242230-1.9791811 .359374$

C $5.6241100 .911916-0.823459$

H $3.9833102 .085732-0.068312$

C $6.269322-0.323291-0.823548$

H $6.279103-2.322053-0.001120$

H $6.0225241 .739000-1.417636$

H $7.170718-0.474876-1.422084$

cis-trans-isomerization distal

$E=-2754.21306511$

Pd -2.216431 -0.601668 -1.649633

P $2.157558-0.060043-0.117642$

P -1.026156 0.365647 -0.019692

C $0.5909820 .304486-0.730076$

C $0.6310680 .582418-2.234398$

H $1.1347201 .530542-2.493136$

H -0.390034 0.665969-2.641004 
H $1.102421-0.222826-2.826100$

C $2.837941-1.584445-0.920570$

H $2.930131-1.241263-1.967538$

C $4.234272-2.013697-0.465521$

H $4.195142-2.3673160 .578497$

H $4.936289-1.164307-0.477776$

C $4.761629-3.140679-1.351296$

H $4.917533-2.749009-2.372858$

H $5.749621-3.470354-0.991108$

C $3.789835-4.314720-1.405971$

H $3.723020-4.774606-0.402834$

H $4.176592-5.096926-2.078539$

C $2.399360-3.870389-1.846883$

H $1.700082-4.720917-1.845932$

H $2.439446-3.509185-2.889933$

C $1.856646-2.756905-0.957491$

H $0.876153-2.413394-1.323819$

H $1.701577-3.1530000 .059312$

C $2.240096-0.1634741 .734888$

H 1.4066890 .4924582 .041286

C 3.5149060 .4056742 .372102

H 3.7118851 .4283572 .023022

H $4.383764-0.2071072 .073663$

C 3.3999260 .4202213 .894631

H 4.3297350 .8204144 .329395

H 2.5962071 .1220894 .181979

C $3.090979-0.9578434 .463014$

H 3.950267 -1.628078 4.280746

H $2.965630-0.9038615 .556042$

C $1.849685-1.5475923 .808683$

H $0.963315-0.9506184 .090853$

H $1.661789-2.5683604 .177847$

C $1.978030-1.5688062 .289690$

H $2.816929-2.2273152 .013908$

H $1.081565-2.0088721 .839816$

C $3.4203671 .237117-0.590102$

H 4.2751051 .0418510 .080451

C $3.9746421 .165994-2.019146$

H $4.4356080 .185562-2.208705$

H $3.1618971 .275683-2.752177$

C $5.0124252 .261543-2.251126$

H $5.3827312 .204148-3.287103$

H $5.8857792 .079180-1.598425$

C $4.4487073 .646208-1.959292$

H $5.2185884 .417657-2.119952$

H $3.6342183 .864658-2.673121$

C $3.9051753 .722918-0.538863$

H $3.4561574 .710681-0.346073$

H 4.740617 3.6160890 .177058

C $2.8743052 .631223-0.275284$

H $1.9746932 .805064-0.888134$

H 2.5325482 .6842380 .770329

C -1.228783 -0.6101921.548142

$\mathrm{H}-0.339261-0.4387842 .175031$

C -2.454702 -0.168463 2.357380

H -3.353519 -0.2387921 .725032$ 
$\mathrm{H}-2.3666650 .8845952 .660471$

C -2.637639 -1.038069 3.597971

$\mathrm{H}-1.781374-0.8871554 .282060$

H $-3.534533-0.7091134 .146986$

C -2.741156 -2.515733 3.243543

$\mathrm{H}-3.650662-2.6764642 .637941$

$\mathrm{H}-2.857348-3.1250244 .154441$

C $-1.524047-2.9681212 .447020$

$\mathrm{H}-0.628730-2.9147673 .092619$

$\mathrm{H}-1.626241-4.0231182 .145846$

C - $1.326401-2.1020561 .208840$

$\mathrm{H}-0.447618-2.4238700 .630790$

$\mathrm{H}-2.180445-2.2401010 .526193$

C -1.418909 2.115011 0.447577

$\mathrm{H}-2.4734582 .1081170 .773743$

C -1.291940 3.020816-0.777821

$\mathrm{H}-0.2519302 .972071-1.142814$

$\mathrm{H}-1.9183112 .643292-1.601743$

C - $1.6364114 .466270-0.439721$

$\mathrm{H}-2.6986884 .528036-0.141496$

$\mathrm{H}-1.5271035 .100543-1.334200$

C - 0.7610294 .9880210 .694292

$\mathrm{H}-1.0384546 .0221960 .954897$

H 0.2891925 .0248920 .350086

C - 0.8522584 .0888181 .921735

$\mathrm{H}-0.1739154 .4467302 .713917$

$\mathrm{H}-1.8737234 .1454192 .338825$

C - 0.5351152 .6349261 .580737

H -0.6370372.009725 2.482132

H 0.5158932 .5545291 .256020

$\mathrm{Cl}-1.964508-2.014395-3.501036$

C $-3.896889-0.087280-0.786239$

C -4.685121-1.139468 -0.294309

C $-4.4298591 .207012-0.776902$

C $-5.960173-0.8959340 .217234$

H -4.308023 -2.165536 -0.318106

C -5.709064 $1.445974-0.268395$

$\mathrm{H}-3.8563912 .043450-1.179894$

C -6.4751300 .4000630 .241626$

$\mathrm{H}-6.556384-1.7310690 .595161$

$\mathrm{H}-6.1076542 .464350-0.275703$

H -7.472707 0.5907980 .644367

trans-2-distal

$E=-2754.21203554$

Cl -3.354331 -2.281290 -2.522482

Pd $-2.828673-0.287638-1.389322$

H 1.5062232 .8609151 .149753

H 1.2688060 .3386021 .942427

$\mathrm{H}-1.253857-3.176051-1.433803$

C 1.4783863 .0305400 .063613

C 2.2230010 .0125121 .497919

C -0.634586 -3.265487 -0.525569

C $2.4063232 .057251-0.661265$

C $1.8179614 .488808-0.225445$

H $0.4447002 .820832-0.253540$ 
$\mathrm{P}-1.143273-0.5405900 .083506$

C -2.858787 $1.607963-0.860176$

P $1.9352580 .281698-0.304796$

C $2.412761-1.4724621 .835195$

C 3.3326900 .8611192 .129569

C - $1.204760-2.3466840 .550085$

C $-0.631795-4.716424-0.055135$

H $0.384471-2.941704-0.778807$

H $3.4359762 .188332-0.281816$

C $2.4045942 .363491-2.164420$

C $1.8319144 .777519-1.719522$

H 2.8090524 .7280070 .201725

H 1.0916455 .1393230 .287747

C $0.349679-0.163602-0.789900$

C -1.514281 0.2841321 .737821

C - $2.0237852 .567979-1.450979$

C -3.884530 $2.054850-0.012376$

C $3.215371-0.641465-1.297268$

H $3.339636-1.8325791 .360632$

H $1.591468-2.0665231 .407118$

C $2.523824-1.7081443 .338659$

C 3.4031930 .6232713 .635571

H 3.1686071 .9299341 .933933

H 4.3052320 .6050751 .678906

$\mathrm{H}-0.534148-2.4126711 .428561$

C -2.608566 -2.797405 0.960927

C -2.028155 -5.167931 0.356845

H $0.057550-4.8275900 .803842$

H -0.238737 -5.364912 -0.855183

H $1.3998172 .155316-2.569315$

H $3.1062951 .710335-2.704508$

C $2.7658433 .819203-2.444153$

H $0.8101334 .659115-2.121565$

H $2.1239865 .822484-1.910611$

C $0.175789-0.343378-2.299890$

$\mathrm{H}-2.6174260 .2797791 .681705$

C -1.136946 -0.475269 3.014553

C -1.085015 1.7433691 .858159

$\mathrm{H}-1.2033532 .248639-2.098985$

C -2.211118 3.929908 -1.206786

C -4.0696613.416306 0.235895

H -4.550794 1.3308220 .467786

H $3.105231-0.185454-2.296066$

C $4.665126-0.398564-0.866375$

C $2.930798-2.132380-1.485570$

H $1.559693-1.4787333 .823196$

H $2.713299-2.7767253 .527408$

C $3.614984-0.8489793 .963347$

H 2.4622100 .9708674 .099889

H 4.2081201 .2373984 .069699

H -3.263910 -2.699470 0.079076

$\mathrm{H}-3.029543-2.1454301 .743471$

C -2.605136 -4.249970 1.428043

$\mathrm{H}-2.010188-6.2118980 .710412$

H -2.686621 -5.138947 -0.528512

H $3.8056414 .003586-2.117913$ 
H $2.7442524 .001778-3.530486$

H - $0.186004-1.346936-2.576252$

$\mathrm{H}-0.5572630 .365462-2.723987$

H $1.116078-0.185659-2.849902$

$\mathrm{H}-0.042068-0.5022743 .127591$

H - $1.469128-1.5203882 .975942$

C -1.746299 0.191545 4.246162

C - 1.7063542 .4088013 .079980

H 0.0098371 .7873491 .958779

$\mathrm{H}-1.3387862 .3034220 .951753$

$\mathrm{H}-1.5517764 .662332-1.681527$

C -3.228638 4.359338 -0.354768

$\mathrm{H}-4.8754313 .7418800 .899778$

H $4.837861-0.8470370 .126600$

H $4.8744000 .677733-0.765990$

C $5.637660-1.022860-1.864336$

C $3.911833-2.744664-2.480628$

H $1.899500-2.273063-1.833535$

H $3.009480-2.662781-0.524317$

H $4.599764-1.1680093 .575989$

H $3.646986-1.0008225 .053922$

H -3.631020 -4.558277 1.687069

H -2.007524 -4.343394 2.354796

$\mathrm{H}-1.446280-0.3616335 .151305$

$\mathrm{H}-2.8465940 .1108984 .185273$

C - 1.3572471 .6594074 .358087

H -2.8023942.4392782.950499

$\mathrm{H}-1.3752083 .4583503 .137541$

H $6.673706-0.864724-1.523930$

H $5.544972-0.498315-2.832492$

C $5.358959-2.507250-2.066402$

H $3.739411-2.300013-3.477506$

H $3.710217-3.822487-2.585239$

$\mathrm{H}-1.8457802 .1222905 .230719$

H -0.2680091 .7334434 .537148$

H $5.559594-3.045273-1.121942$

H $6.049536-2.928101-2.814525$

H -3.368948 $5.424614-0.156183$

3-distal

$E=-2889.30577871$

Pd $-2.512729-0.891288-0.850324$

C -3.787087 $0.562014-0.382173$

N -4.131921 -1.987976 -1.787829

P -0.7553370 .0791310 .283612$

Cl - $1.234868-2.777314-1.709355$

P $2.3684150 .126818-0.409516$

C $0.6853960 .149145-0.740004$

C 2.7824420 .1597311 .401544

C $3.199955-1.303086-1.252926$

C $3.2954931 .579783-1.146836$

C - $0.542194-0.9391761 .831879$

C -1.146216 1.7764790 .950446

C -5.164322 -2.423360 -0.859338

C -4.670474 -1.391765 -3.000609

H -3.526668 -2.779178 -2.037719 
C $0.3884170 .243764-2.232558$

$\mathrm{H}-0.609057-0.173381-2.439107$

H $1.079089-0.352175-2.854295$

H $0.3993461 .278543-2.626601$

C $2.5940222 .889055-0.789015$

C $3.5512881 .517029-2.657473$

H $4.2848611 .564786-0.656792$

C $4.3513562 .732284-3.121341$

H $4.0976890 .601667-2.928042$

H $2.5924591 .482225-3.197566$

C $3.6665984 .041375-2.749128$

H $5.3544992 .701647-2.657827$

H $4.5119422 .676608-4.210152$

C $3.3855084 .105707-1.253423$

H $2.7116384 .119342-3.299256$

H $4.2794684 .900633-3.065871$

H $4.3422704 .153084-0.701539$

H $2.8354235 .026901-1.001236$

H $1.6023522 .886225-1.268019$

H 2.3976452 .9517950 .292640

C $4.712666-1.411350-1.049883$

C $2.493646-2.643047-1.048505$

H $3.037414-1.042514-2.314644$

C $3.088577-3.697183-1.975445$

H $1.412116-2.533389-1.229921$

H $2.614820-2.979413-0.006191$

C $4.596331-3.826297-1.786750$

H $2.590259-4.664593-1.805715$

H $2.870285-3.419830-3.022573$

C $5.298941-2.484457-1.965543$

H $5.013881-4.569448-2.485341$

H $4.802167-4.207716-0.769660$

H $5.190867-2.154809-3.015040$

H $6.381265-2.585338-1.782374$

H $4.931203-1.675935-0.001525$

H $5.212193-0.447026-1.235563$

C $2.906057-1.2260972 .044498$

C 3.9995911 .0103941 .787464

H 1.8959130 .6524741 .832415

C $3.020740-1.1192763 .561163$

H $3.804992-1.7302071 .655183$

H $2.054534-1.8589321 .773778$

C $4.202362-0.2517933 .971393$

H $2.087426-0.6874083 .966404$

H $3.107072-2.1272033 .997717$

C 4.1281171 .1150643 .305197

H $5.140656-0.7521603 .670833$

H $4.244533-0.1446455 .067106$

H 5.0134281 .7196953 .559729

H 3.2533701 .6652223 .696986

H 4.9157360 .5561691 .370468

H 3.9288852 .0226721 .367598

C -0.341895-2.423095 1.525009

C - $1.745812-0.7826292 .765241$

H $0.346609-0.5566272 .362025$

C $-0.153201-3.2462332 .793896$ 
H -1.222501 -2.788206 0.973093

H $0.489271-2.5686900 .822489$

C -1.325533 -3.066109 3.750265

$\mathrm{H}-0.031027-4.3092772 .529904$

H 0.778506 -2.945100 3.306605

C - $1.565932-1.5912404 .046423$

$\mathrm{H}-1.158416-3.6268054 .684546$

$\mathrm{H}-2.234188-3.4915913 .287488$

$\mathrm{H}-2.448539-1.4652434 .694874$

H - $0.706318-1.1863254 .612334$

$\mathrm{H}-1.9355180 .2720503 .012459$

$\mathrm{H}-2.638668-1.1440282 .226431$

C -0.206275 2.2974812.035252

C -1.211455 2.776077 -0.202417

$\mathrm{H}-2.1617841 .6845961 .372131$

C - 0.6422213 .6754642 .529028

H -0.147353 1.5997662 .886036

H 0.8113472 .3744061 .613844

C - 0.7556104 .6749191 .383256

H 0.0608274 .0438253 .294900

$\mathrm{H}-1.6239603 .5808173 .026915$

C -1.663599 4.149543 0.276949

H 0.2519274 .8590760 .965815

$\mathrm{H}-1.1177295 .6465641 .757453$

$\mathrm{H}-1.6871324 .857201-0.568178$

$\mathrm{H}-2.7014504 .0747960 .647820$

H -0.210220 $2.849247-0.655060$

$\mathrm{H}-1.8840192 .404343-0.985897$

C -4.100027 1.526539-1.356093

C - 4.5072380 .6159000 .821667

C $-5.0693622 .506541-1.134023$

$\mathrm{H}-3.5711821 .519337-2.314690$

C -5.763040 2.5482060 .075105

$\mathrm{H}-5.2806943 .245287-1.912562$

C -5.4799671.5930311.049695

$\mathrm{H}-6.0197601 .6056152 .001103$

$\mathrm{H}-4.312133-0.1168831 .608646$

H -5.887428 $-3.105599-1.343858$

H -5.705938 $-1.548243-0.472424$

H -3.846582 -1.156426 -3.688260

H -5.196517 -0.458826 -2.753008

H $-4.698228-2.942006-0.010329$

H $-5.377861-2.073071-3.508848$

H -6.520021 3.3157990 .253896

$\mathbf{L} 2={ }^{C y}$ JohnPhos

trans-3

$E=-2227.18623824$

Pd -0.020959 $0.990052-1.003038$

C $1.980505-1.6359610 .042637$

H $1.852469-2.7144180 .262451$

C $2.450026-1.494182-1.414137$

H $2.609129-0.422625-1.612334$

H $1.680416-1.820584-2.127070$

C $3.746338-2.262807-1.649780$ 
H 3.559952 -3.344750 -1.516207 H $4.069512-2.130965-2.695126$ C $4.843130-1.813384-0.692084$ H $5.116428-0.769129-0.924857$ H $5.753320-2.417859-0.836377$ C $4.373185-1.8833000 .755889$ H 4.213662 -2.939554 1.043420 H 5.151666 -1.4910861.429703 C - $0.791058-2.422235-0.181358$ $\mathrm{H}-0.442953-3.1623640 .564301$ C -0.563886 -3.034333-1.567107 $\mathrm{H}-0.710210-2.276759-2.356688$ H $0.480119-3.363926-1.664092$ C -1.487634 -4.220040-1.833397 $\mathrm{H}-1.212491-5.049763-1.157769$ $\mathrm{H}-1.324483-4.589033-2.858621$ C -2.950254 -3.864664 -1.609067 H -3.594247 -4.736276 -1.806703 H -3.255963 -3.084272 -2.331090 C -3.157019-3.354023 -0.190449 $\mathrm{H}-4.211350-3.084497-0.018424$ $\mathrm{H}-2.923465-4.1660200 .521431$ C -2.269569-2.152354 0.111773 H -2.379607 -1.867105 1.166572 H -2.627957 -1.279357 -0.459328 $\mathrm{Cl}-0.1897303 .071736-2.126804$ C $1.5906181 .709184-0.043806$ C 1.7420271 .8989551 .334954 C $2.6743722 .059053-0.867749$ C 2.9284262 .4052431 .875296 H 0.9471281 .6265382 .031159 C $3.8650102 .546824-0.331500$ H $2.5800721 .966709-1.952140$ C 4.0009092 .7203821 .047178 H 3.0111872 .5367632 .957662 H 4.6901022 .806917 -1.000510 H 4.9322353 .1074251 .467805 $\mathrm{N}-1.6117310 .267166-2.354099$ $\mathrm{H}-1.804188-0.696778-2.094153$ C -2.854488 $1.023264-2.228115$ H -3.257682 $0.900539-1.214741$ $\mathrm{H}-2.6283882 .087289-2.380274$ $\mathrm{H}-3.6072130 .691589-2.967271$ C $-1.0900900 .275986-3.719498$ $\mathrm{H}-0.7901221 .303667-3.966946$ H -0.197524 -0.363267 -3.778603 H - $1.844049-0.079312-4.446072$ P $0.262149-0.9105970 .197409$ C - $0.133510-0.7945051 .996451$ C $0.514504-1.7048952 .845881$ C - 1.1175570 .0644192 .545461 C $0.274943-1.7402944 .214961$ H $1.225208-2.4179992 .425424$ C - 1.3381020 .0147253 .932551 C $-0.646636-0.8564364 .766109$ H $0.808243-2.4567654 .843697$ 
$\mathrm{H}-2.0868430 .6885744 .355088$

$\mathrm{H}-0.842602-0.8546275 .840817$

C -2.051610 0.965618 1.799595

C - 1.6708552 .1764561 .206804

C -3.417692 0.6393491 .821199

C -2.6251513.0286340.650373

H -0.629328 2.489701 1.202434

C -4.371033 1.4895951 .265763

H -3.735682 -0.292542 2.296039

C -3.975253 2.692431 0.680398

$\mathrm{H}-2.2884493 .9512310 .174156$

H -5.4277131.212859 1.297955

$\mathrm{H}-4.7189243 .3632600 .243611$

C $3.084265-1.0918090 .956971$

H $2.787748-1.1045342 .013984$

H $3.271092-0.0393920 .707495$

cis-2-distal

$E=-2092.10510868$

Pd $1.778532-0.774470-1.151402$

C - 0.6764601 .4070480 .120947

$\mathrm{H}-1.7430711 .3906860 .391802$

C 0.0631262 .2474321 .169482

H 1.1493802 .1999370 .995217

H -0.1145981 .8459442 .178954$

C -0.395663 3.701462 1.112761

$\mathrm{H}-1.4616343 .7592431 .399961$

H 0.1636094 .2907061 .856982

C - $0.2187504 .292071-0.280357$

H $0.8571884 .308991-0.529860$

$\mathrm{H}-0.5635875 .338475-0.300772$

C - $0.9655733 .464819-1.319237$

$\mathrm{H}-2.0507203 .524481-1.123391$

H $-0.8048593 .872674-2.330259$

C - $0.5297042 .005558-1.281357$

$\mathrm{H}-1.1032091 .410688-2.009352$

H $0.5263811 .925582-1.586800$

C $0.056454-0.9065691 .834015$

H $0.793376-0.1950662 .249486$

C $0.661470-2.3082271 .930307$

H -0.041084 -3.037756 1.490379

H $1.585221-2.3674631 .330836$

C $0.927461-2.6822043 .384761$

H $1.700863-2.0078663 .795162$

H $1.343403-3.7007333 .439995$

C - $0.338080-2.5751664 .228575$

$\mathrm{H}-0.118872-2.8068995 .283134$

$\mathrm{H}-1.063583-3.3359033 .887755$

C $-0.972274-1.1939624 .109391$

$\mathrm{H}-1.914491-1.1510534 .679441$

H $-0.301190-0.4416694 .561658$

C -1.228087 -0.817176 2.652337

$\mathrm{H}-1.6671410 .1905972 .589512$

H -1.967116 -1.514215 2.219668

Cl $3.449033-1.473908-2.557607$

C $2.8063190 .541800-0.140457$ 
C $3.1383551 .747154-0.765609$

C 3.2477810 .2893681 .159799

C $3.8584662 .716611-0.065942$

H $2.8436871 .932678-1.800321$

C 3.9708611 .2646701 .851251

H $3.037699-0.6667981 .643326$

C 4.2650392 .4858321 .248082

H $4.1084693 .659098-0.560396$

H 4.3074661 .0605412 .871222

H 4.825909 3.248459 1.793198

P $-0.080210-0.3382900 .082080$

C -1.326195 -1.437831-0.721148

C - $0.752695-2.645023-1.168622$

C -2.696027 -1.211741 -0.984029

C - $1.488485-3.609200-1.847103$

H $0.311510-2.834283-0.981548$

C -3.419602 -2.189382 -1.683223

C $-2.834828-3.376733-2.110085$

$\mathrm{H}-1.002308-4.528951-2.178669$

$\mathrm{H}-4.476284-1.999541-1.886599$

H -3.430662 -4.115068 -2.651459

C -3.456070 $0.010489-0.597119$

C -3.950283 0.181811 0.701349

C -3.753871 $0.978618-1.563690$

C -4.675611 1.322320 1.040022

H -3.764135 -0.588287 1.450560

C -4.479914 2.119124-1.226038

H -3.400791 $0.837366-2.587968$

C -4.9320612.300395 0.080112

$\mathrm{H}-5.0481021 .4440612 .059913$

$\mathrm{H}-4.6931242 .870906-1.989531$

H -5.497078 3.1966130 .346280

cis-trans isomerization distal

$E=-2092.09910304$

Cl $4.035745-2.0946340 .244815$

Pd $2.315242-0.5600680 .075482$

H $0.808296-3.0495000 .613023$

C -0.265151 -3.004094 0.351882

P $0.089294-0.262327-0.025900$

C $2.4729571 .378508-0.085550$

C - $0.481260-1.896208-0.681954$

C $-0.710443-4.355791-0.196250$

$\mathrm{H}-0.797979-2.7700121 .287728$

C $-0.5641821 .047803-1.166730$

C 2.5681692 .1984601 .042709

C $2.6757991 .924528-1.357768$

H - $1.559479-1.796886-0.889284$

C $0.244003-2.228416-1.991402$

C - $0.009273-4.685445-1.508879$

$\mathrm{H}-1.804443-4.340314-0.358936$

$\mathrm{H}-0.518698-5.1410620 .552131$

H $0.2815421 .147495-1.870832$

C - $1.8060400 .702040-1.992349$

C $-0.7112402 .397475-0.458281$

H 2.4078091 .7920302 .043196 
C 2.8589553 .5563460 .894056

C $2.9638043 .283301-1.496746$

H $2.6224961 .289808-2.246596$

H $1.332187-2.257570-1.801102$

H $0.083561-1.437438-2.741464$

C - $0.211212-3.578256-2.536717$

H $-0.368459-5.648728-1.905014$

H $1.071690-4.806513-1.318619$

H -2.673359 $0.555081-1.335255$

$\mathrm{H}-1.661039-0.240954-2.539313$

C -2.112641 $1.821952-2.983800$

C - $1.0344883 .501974-1.458083$

$\mathrm{H}-1.5190022 .3266880 .287097$

H 0.2070172 .6425760 .093087

H 2.9309884 .1893891 .782361

C $3.0488454 .104878-0.373231$

H $3.1266093 .699218-2.494625$

H $0.338973-3.807130-3.463210$

$\mathrm{H}-1.280041-3.520727-2.813546$

H -3.024368 $1.570789-3.549532$

$\mathrm{H}-1.2943041 .888518-3.723794$

C $-2.2686843 .167886-2.286473$

H -0.165506 3.642915 -2.125038

$\mathrm{H}-1.1694604 .455908-0.923447$

$\mathrm{H}-2.4648073 .961818-3.025146$

H -3.152848 3.129319-1.625135

H $3.2689715 .168963-0.485167$

C -0.585387 -0.054195 1.680150

C $0.392605-0.0677812 .688198$

C - 1.9346870 .1432342 .057397

C 0.0769540 .1424264 .026417

H $1.432028-0.2541422 .399427$

C - 2.2308800 .3837683 .406343

C - 1.2431680 .3901314 .386008

H 0.8674680 .1182804 .779472

H -3.275471 0.5419243 .685038

$\mathrm{H}-1.5110230 .5721415 .429384$

C -3.0971300.076469 1.133544

C -3.879820 1.213821 0.894391

C -3.493343 -1.141732 0.567952

C -4.9935341.1480220.060339

$\mathrm{H}-3.5990912 .1629791 .357276$

C -4.608036 - $1.209161-0.265565$

$\mathrm{H}-2.933033-2.0479730 .804302$

C $-5.353068-0.061650-0.533074$

$\mathrm{H}-5.5824482 .048343-0.129834$

$\mathrm{H}-4.900199-2.167119-0.702165$

H -6.223356 -0.113050 -1.191197

trans-2-distal

$E=-2092.10004044$

Cl $0.147917-2.3002322 .718495$

Pd $1.514953-1.3132161 .103370$

$\mathrm{H}-0.716156-2.8465920 .035138$

C - $1.574326-2.222228-0.264668$

P 0.0602350 .0244090 .087959 
C $3.044641-0.6974420 .015306$

C - $1.048023-0.983601-0.991414$

C -2.512918 -3.023531-1.157345

$\mathrm{H}-2.060877-1.9464870 .680272$

C $0.7614851 .359441-0.984381$

C $4.074342-0.1041830 .764852$

C $3.286017-0.973157-1.337123$

$\mathrm{H}-1.897343-0.330546-1.253108$

C $-0.334769-1.402391-2.280831$

C - $1.837008-3.419930-2.463942$

$\mathrm{H}-3.411239-2.421030-1.377547$

H -2.859115 -3.917799 -0.614951

H $1.4100280 .796948-1.678068$

C - $0.2801552 .132917-1.790046$

C $1.6539552 .333334-0.214873$

H 3.9151580 .1381121 .821516

C 5.3101930 .1882920 .185119

C $4.521158-0.678172-1.918323$

H $2.511486-1.436531-1.952333$

H $0.532256-2.030946-2.013400$

H $0.055936-0.531763-2.829104$

C - $1.275183-2.198724-3.180917$

$\mathrm{H}-2.541583-3.957898-3.118558$

$\mathrm{H}-1.012151-4.122406-2.247845$

H -0.906026 $2.709684-1.088554$

H - $0.9555561 .457015-2.336216$

C $0.4014953 .092753-2.762305$

C $2.3520693 .286017-1.180021$

H 1.0353952 .9106090 .493907

H 2.3987191 .7827780 .375754

H 6.0976090 .6463740 .789726

C $5.535236-0.092663-1.161829$

H $4.691264-0.912357-2.972853$

$\mathrm{H}-0.744181-2.496620-4.099488$

$\mathrm{H}-2.107058-1.544018-3.498143$

$\mathrm{H}-0.3596403 .657269-3.325172$

H $0.9670792 .505107-3.507690$

C $1.3489934 .044603-2.041476$

H $3.0346272 .702746-1.823433$

H $2.9848463 .989711-0.615994$

H $1.8705764 .688615-2.767697$

H $0.7576314 .719321-1.396200$

H $6.4988190 .142688-1.620038$

C - 0.8750770 .9305641 .387546

C -0.059949 1.3407902 .456964

C - 2.2341101 .3127741 .387520

C - 0.5436442 .1371373 .485864

H 0.9789891 .0032502 .481559

C -2.706148 2.110704 2.441305

C - 1.8784602 .5310703 .475310

H 0.1197692 .4328874 .301135

H -3.7616712.3928162.438646

H -2.281792 3.150464 4.279732

C -3.265151 0.8863510 .396779

C -3.550075 $1.637740-0.749223$

C $-4.050448-0.2392740 .675234$ 
C -4.556815 1.240269-1.627472

$\mathrm{H}-2.9815422 .546257-0.951390$

C -5.062216 -0.632661 -0.198201

H $-3.860309-0.8075601 .588645$

C -5.309840 0.098412 -1.359182

H -4.760552 $1.834229-2.521654$

$\mathrm{H}-5.661130-1.5169310 .031767$

H -6.099832 -0.212935-2.046521

\section{3-distal}

$E=-2227.19802270$

Pd 1.9868790 .5580680 .052637

C -1.092044-1.120161-0.901933

H -2.166770 -0.873758 -0.899405

C - $0.666564-1.262567-2.365654$

H $0.422672-1.417912-2.415148$

H $-0.885248-0.338954-2.920666$

C - $1.385952-2.430328-3.036028$

$\mathrm{H}-2.465203-2.199204-3.096042$

$\mathrm{H}-1.031374-2.534618-4.074501$

C -1.197587 -3.732411-2.268801

$\mathrm{H}-0.129184-4.013255-2.281440$

$\mathrm{H}-1.746226-4.551133-2.762428$

C -1.653011-3.574573-0.823903

$\mathrm{H}-2.739419-3.376959-0.802001$

H - $1.488355-4.508561-0.262465$

C -1.060681 1.786004-0.999284

$\mathrm{H}-2.0099061 .384353-1.401291$

C $-0.1870162 .240850-2.175480$

H $0.7654852 .615012-1.767311$

H $0.0610451 .397827-2.837333$

C $-0.8758603 .349028-2.965582$

$\mathrm{H}-1.8017842 .957109-3.426682$

$\mathrm{H}-0.2265103 .669509-3.796472$

C - $1.2167204 .533566-2.069707$

$\mathrm{H}-1.7403775 .315652-2.643475$

H $-0.2788774 .983108-1.699607$

C -2.056233 4.093826 -0.876102

$\mathrm{H}-2.2547764 .948161-0.208897$

H -3.043117 $3.742734-1.234250$

C -1.372423 $2.979073-0.091204$

$\mathrm{H}-1.9961512 .6745360 .762916$

$\mathrm{H}-0.4244583 .3457470 .334409$

Cl 2.0673952 .9631190 .405750

C $2.238231-1.4054270 .009964$

C $2.193736-2.1201421 .217438$

C $2.594693-2.103119-1.152427$

C $2.479316-3.4858881 .258301$

H $1.905922-1.6098122 .141898$

C $2.873991-3.471806-1.115916$

H $2.648821-1.578334-2.110792$

C $2.812993-4.1702820 .089220$

H $2.428179-4.0217862 .210206$

H $3.139402-3.995357-2.038816$

H $3.026454-5.2414870 .117837$

N 4.1279860 .8050310 .179734 


$\begin{array}{llll}\text { H } 4.069412 & 1.824639 & 0.288881 \\ \text { C } 4.739325 & 0.226088 & 1.368600 \\ \text { H } 4.203976 & 0.575952 & 2.261727 \\ \text { H } 4.665718 & -0.869776 & 1.328152 \\ \text { H } 5.803816 & 0.510804 & 1.453973 \\ \text { C } 4.851771 & 0.486237 & -1.042798 \\ \text { H } 4.801652 & -0.595554 & -1.230279 \\ \text { H } 4.390174 & 1.010489 & -1.890698 \\ \text { H } 5.914121 & 0.783384 & -0.972492 \\ \text { P }-0.298564 & 0.349625 & -0.082979 \\ \text { C }-0.989333 & 0.395869 & 1.630481 \\ \text { C }-0.071646 & 0.793865 & 2.615639 \\ \text { C }-2.302481 & 0.050715 & 2.032633 \\ \text { C }-0.410086 & 0.834088 & 3.964371 \\ \text { H } 0.932662 & 1.091792 & 2.300585 \\ \text { C }-2.616224 & 0.071314 & 3.399452 \\ \text { C }-1.688034 & 0.455749 & 4.361489 \\ \text { H } 0.330524 & 1.157261 & 4.699205 \\ \text { H }-3.629971 & -0.204080 & 3.700349 \\ \text { H }-1.970357 & 0.469331 & 5.416872 \\ \text { C }-3.408252 & -0.340293 & 1.115346 \\ \text { C }-3.983893 & 0.573501 & 0.223090 \\ \text { C }-3.943737 & -1.633176 & 1.185156 \\ \text { C }-5.027212 & 0.188055 & -0.615882 \\ \text { H }-3.617393 & 1.599706 & 0.195346 \\ \text { C }-4.986223 & -2.019825 & 0.346047 \\ \text { H }-3.518124 & -2.348782 & 1.892632 \\ \text { C }-5.523511 & -1.113677 & -0.566992 \\ \text { H }-5.459076 & 0.914726 & -1.308188 \\ \text { H }-5.378359 & -3.038014 & 0.403786 \\ \text { H }-6.337534 & -1.417408 & -1.229195 \\ \text { C }-0.909720 & -2.433143 & -0.139689 \\ \text { H }-1.245376 & -2.320151 & 0.902334 \\ \text { H } 0.156606 & -2.686753 & -0.092824\end{array}$

\subsubsection{Energies and Coordinates for Other Aryl Chlorides and Amines}

Energies in Hartree at the PBE0-D3/def2tvp - SDD level with PCM (THF), Coordinates in Angstrom. These calculations were performed with the Stuttgart Dresden basis set MWB28 and the corresponding ECP for Palladium. ${ }^{[11]}$

Table S18. Barriers for oxidative addition (OA) and reductive elimination (RE) for different aryl chlorides and amines; free energies $\mathrm{kJ} / \mathrm{mol}$.

\begin{tabular}{ccccc}
\hline Aryl chlorides & $\Delta \mathrm{G}^{\ddagger}(\mathrm{OA})$ & $\Delta \mathrm{G}^{\ddagger}(\mathrm{RE})$ & Amines & $\Delta \mathrm{G}^{\ddagger}(\mathrm{RE})$ \\
\hline \hline $\mathrm{Ph}-\mathrm{Cl}$ & 47 & 50 & $\mathrm{HNMe}_{2}$ & 50 \\
$p-\mathrm{CN}-\mathrm{Ph}-\mathrm{Cl}$ & 47 & 42 & $\mathrm{HNEt}_{2}$ & 55 \\
$p-\mathrm{Me}-\mathrm{Ph}-\mathrm{Cl}$ & 50 & 51 & piperidine & 58 \\
$p-\mathrm{OMe}-\mathrm{Ph}-\mathrm{Cl}$ & 54 & 55 & ${ }^{t} \mathrm{BuNH}_{2}$ & 64 \\
$m-(\mathrm{OMe})_{2}-\mathrm{Ph}-\mathrm{Cl}$ & 52 & 52 & ${ }^{i} \mathrm{PrNH}_{2}$ & 56 \\
& & & 2,6-diisopropylaniline & 67 \\
\hline
\end{tabular}

Table S19. SCF energies and enthalpy/free energy corrections for other aryl chlorides and amines. 


\section{ARYL CHLORIDES}

\begin{tabular}{|c|c|c|c|c|c|}
\hline $\begin{array}{c}\mathrm{ArCl}= \\
\text { Chlorobenzene }\end{array}$ & Corr(G) & Corr(H) & $\mathrm{E}(\mathrm{PCM})$ & $\begin{array}{c}\Delta \mathbf{G}(\mathrm{PCM}) / \\
\mathrm{E}_{\mathrm{H}}\end{array}$ & $\begin{array}{c}\Delta \mathrm{G}(\mathrm{PCM}) / \mathrm{kJ} / \\
\mathrm{mol}\end{array}$ \\
\hline L1-Pd(ArCl) & 0.86731 & 0.993354 & -2755.348777 & & \\
\hline $\begin{array}{l}\text { TS (oxidative } \\
\text { addition) }\end{array}$ & 0.867732 & 0.992854 & -2755.331178 & 0.018020 & 47.312 \\
\hline L1-Pd(Ar)-Cl & 0.8716 & 0.995269 & -2755.383774 & & \\
\hline $\begin{array}{c}\text { L1-Pd(Ar)- } \\
\mathrm{Cl}\left(\mathrm{HNMe}_{2}\right)\end{array}$ & 0.947141 & 1.078054 & -2429.709788 & & \\
\hline $\begin{array}{l}\text { TS(reductive } \\
\text { elimination) }\end{array}$ & 0.946118 & 1.076641 & -2429.689716 & 0.019050 & 50.015 \\
\hline L1-Pd-ArNMe ${ }_{2}$ & 0.947688 & 1.079946 & -2429.741291 & & \\
\hline
\end{tabular}

$\mathrm{ArCl}=p-$

Chlorotoluene

\begin{tabular}{|c|c|c|c|c|c|}
\hline L1-Pd(ArCl) & 0.890638 & 1.022845 & -2794.633108 & \multirow{3}{*}{0.019167} & \multirow[b]{2}{*}{50.322} \\
\hline $\begin{array}{l}\text { TS (oxidative } \\
\text { addition) }\end{array}$ & 0.892909 & 1.021972 & -2794.616212 & & \\
\hline L1-Pd(Ar)-Cl & 0.894868 & 1.024446 & -2794.671481 & & \multirow{4}{*}{50.819} \\
\hline $\begin{array}{l}\text { L1-Pd(Ar)- } \\
\mathrm{Cl}\left(\mathrm{HNMe}_{2}\right)\end{array}$ & 0.970782 & 1.107175 & -2468.993854 & \multirow{4}{*}{0.019356} & \\
\hline $\begin{array}{l}\text { TS(reductive } \\
\text { elimination) }\end{array}$ & 0.969781 & 1.105811 & -2468.973497 & & \\
\hline $\mathrm{L} 1-\mathrm{Pd}-\mathrm{ArNMe}_{2}$ & 0.973179 & 1.10911 & \multirow[t]{2}{*}{-2469.026661} & & \\
\hline $\begin{array}{c}\mathrm{ArCl}=1-\text { Chloro- } \\
\text { 3,5- } \\
\text { dimethoxybenzene }\end{array}$ & & & & & \\
\hline L1-Pd(ArCl) & 0.925358 & 1.063931 & -2984.248507 & \multirow{4}{*}{0.019782} & \multirow{4}{*}{51.938} \\
\hline $\begin{array}{l}\text { TS (oxidative } \\
\text { addition) }\end{array}$ & 0.927242 & 1.063312 & -2984.230609 & & \\
\hline L1-Pd(Ar)-Cl & 0.930928 & 1.065957 & -2984.283204 & & \\
\hline $\begin{array}{l}\text { L1-Pd(Ar)- } \\
\mathrm{Cl}\left(\mathrm{HNMe}_{2}\right)\end{array}$ & 1.00573 & 1.148424 & -2658.609352 & & \\
\hline $\begin{array}{l}\text { TS(reductive } \\
\text { elimination) }\end{array}$ & 1.005823 & 1.14725 & -2658.589578 & \multirow[t]{2}{*}{0.019867} & \multirow[t]{2}{*}{52.160} \\
\hline L1-Pd-ArNMe ${ }_{2}$ & 1.008345 & 1.150671 & -2658.642717 & & \\
\hline
\end{tabular}

$\mathrm{ArCl}=p-$

Chlorobenzonitrile

\begin{tabular}{cccccc}
\hline \hline L1-Pd(ArCl) & 0.863971 & 0.994061 & -2847.5223 & & \\
TS (oxidative & 0.865077 & 0.993655 & -2847.50555 & 0.017859 & 46.888 \\
addition) & 0.86763 & 0.995915 & -2847.54653 & & \\
L1-Pd(Ar)-Cl & 0.8678 & & \\
L1-Pd(Ar)- & 0.943064 & 1.078748 & -2521.88259 & & \\
Cl(HNMe $)$ & & & & \\
TS(reductive & 0.942089 & 1.077383 & -2521.86558 & 0.016036 & 42.103 \\
elimination) & & & & \\
L1-Pd-ArNMe $_{2}$ & 0.944395 & 1.080643 & -2521.91268 & & \\
\hline
\end{tabular}

$\mathrm{ArCl}=1-$ Chloro-4-

methoxybenzene 


\begin{tabular}{cccccc}
\hline \hline L1-Pd(ArCl) & 0.895525 & 1.028783 & -2869.796511 & & \\
TS (oxidative & 0.898446 & 1.028233 & -2869.778748 & 0.020684 & 54.305 \\
addition) & 0.90114 & 1.030643 & -2869.834925 & & \\
L1-Pd(Ar)-Cl & 0.97656 & 1.113299 & -2544.157322 & & \\
L1-Pd(Ar)- & & & & \\
Cl(HNMe 2 ) & & & & \\
TS(reductive & 0.976025 & 1.112142 & -2544.135674 & 0.021113 & 55.432 \\
elimination) & & & & \\
L1-Pd-ArNMe $_{2}$ & 0.978989 & 1.115291 & -2544.189849 & & \\
\hline
\end{tabular}

\section{AMINES}

\begin{tabular}{|c|c|c|c|c|c|}
\hline $\begin{array}{c}\text { L1-Pd(Ar)- } \\
\mathrm{Cl}\left(\mathrm{HNR}_{2}\right)\end{array}$ & 1.00392 & 1.138094 & -2508.277761 & & \\
\hline $\begin{array}{l}\text { TS (reductive } \\
\text { elimination) }\end{array}$ & 1.003609 & 1.136824 & -2508.256814 & 0.020947 & 54.996 \\
\hline L1-Pd-ArNR 2 & 1.002786 & 1.139213 & -2508.306918 & & \\
\hline
\end{tabular}

piperidine

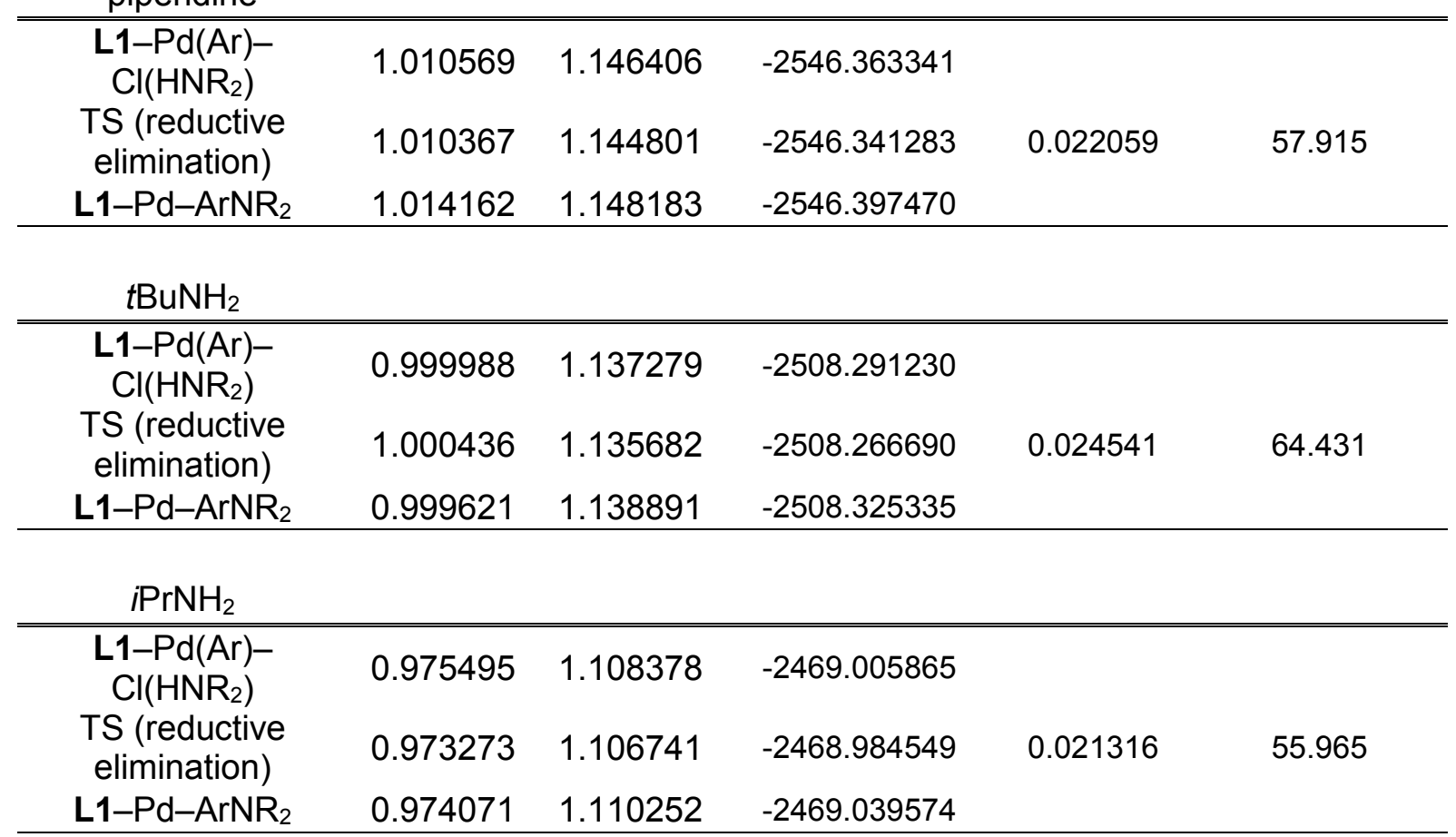

2,6-

diisopropylaniline

\begin{tabular}{|c|c|c|c|c|c|}
\hline $\begin{array}{c}\text { L1-Pd(Ar)- } \\
\mathrm{Cl}\left(\mathrm{HNR}_{2}\right)\end{array}$ & 1.132217 & 1.283669 & -2817.744148 & & \\
\hline $\begin{array}{l}\text { TS (reductive } \\
\text { elimination) }\end{array}$ & 1.131358 & 1.28243 & -2817.718707 & 0.025441 & 66.795 \\
\hline L1-Pd-ArNR 2 & 1.131489 & 1.284612 & -2817.757877 & & \\
\hline
\end{tabular}

\section{Arylchlorides}


$\mathrm{ArCl}=$ Chlorobenzene

L1-Pd(ArCl)

$E=-2755.34877679$

Pd $1.632059-0.1809620 .781421$

P -2.114147 -0.203711 -0.118759

P $0.6589330 .421339-1.230913$

C -1.096177 0.243169-1.431275

C -1.654171 $0.250932-2.840781$

$\mathrm{H}-1.3037501 .128644-3.414210$

$\mathrm{H}-2.7540120 .292091-2.872530$

H -1.370722 -0.637753 -3.437368

C -3.824580 $0.417559-0.461383$

$\mathrm{H}-4.114333-0.148398-1.365847$

C -4.864608 0.0999820 .614991

$\mathrm{H}-4.6194690 .6574711 .534835$

$\mathrm{H}-4.849167-0.9677930 .881781$

C -6.261973 0.4995640 .147287

$\mathrm{H}-6.545735-0.131814-0.714234$

H -6.997343 0.2894710 .940729

C -6.322108 $1.967065-0.261228$

H -6.157818 2.5968290 .632029

H -7.326866 2.219369-0.636849

C -5.260867 2.298728 -1.304601

H -5.283924 $3.372886-1.549188$

H -5.488957 $1.762037-2.243632$

C -3.866894 $1.903676-0.828800$

$\mathrm{H}-3.1078882 .131811-1.589940$

$\mathrm{H}-3.6027782 .5059330 .054670$

C - 1.4826650 .4010031 .499349

$\mathrm{H}-0.4182830 .0526861 .437086$

C -2.085964 -0.207272 2.766977

H -2.148935 -1.302422 2.705282

$\mathrm{H}-3.1173670 .1598892 .904098$

C -1.241929 0.1851583 .978350

$\mathrm{H}-1.690929-0.2298874 .895281$

H $-0.250069-0.2875453 .877351$

C -1.084048 1.6967474 .104242

$\mathrm{H}-2.0645432 .1425264 .354102$

$\mathrm{H}-0.4135641 .9399434 .945094$

C - 0.5692572 .3224552 .812838

H 0.4656491 .9881782 .617489

H - 0.5353543 .4203792 .903390

C - 1.4314471 .9217481 .622770

$\mathrm{H}-2.4539202 .3149201 .763010$

$\mathrm{H}-1.0440052 .3600300 .694472$

C -2.355112 -2.033482 0.150220

$\mathrm{H}-3.109801-2.1682430 .946136$

C -2.874958 -2.684852-1.135135

$\mathrm{H}-3.866623-2.283182-1.399163$

H -2.198641 -2.415461-1.964572

C -2.952126 -4.204172 -1.022832

$\mathrm{H}-3.295071-4.630212-1.979728$

H -3.714934 -4.475239-0.270290

C -1.612714 -4.803998 -0.614797 
H -1.693625 -5.899175 -0.522623

$\mathrm{H}-0.869515-4.610197-1.409299$

C - $1.124953-4.1898500 .690202$

$\mathrm{H}-0.138688-4.5938880 .969819$

$\mathrm{H}-1.818162-4.4722051 .503974$

C - $1.038460-2.6707740 .602377$

$\mathrm{H}-0.245871-2.385498-0.106597$

H $-0.715860-2.2669061 .572052$

C $1.399206-0.612237-2.610141$

H $0.933696-0.260575-3.550336$

C $2.912815-0.437968-2.735669$

H $3.373156-0.679142-1.760138$

H $3.1667700 .611687-2.947500$

C $3.503294-1.331306-3.823844$

H $3.122610-1.003194-4.808376$

H $4.598208-1.205562-3.858878$

C $3.140821-2.796339-3.614349$

H $3.611062-3.156268-2.680798$

H $3.549690-3.416903-4.428516$

C $1.630956-2.971326-3.509525$

H $1.165868-2.710934-4.477908$

H $1.374913-4.026532-3.316043$

C $1.048658-2.083891-2.416149$

H - $0.043940-2.201744-2.361506$

H $1.443976-2.403340-1.433930$

C $1.1506922 .138241-1.842026$

H $1.3849672 .027894-2.918288$

C $2.4111512 .624096-1.119787$

H $2.1829382 .698585-0.040665$

H $3.2164611 .877874-1.188172$

C $2.8833343 .973826-1.650565$

H $3.2067883 .857246-2.701444$

H $3.7706644 .311295-1.089620$

C $1.7786715 .022013-1.579769$

H $2.1186235 .974902-2.017900$

H $1.5499885 .231475-0.518695$

C $0.5096764 .536627-2.272206$

H -0.294983 5.283341 -2.166810

H $0.7005204 .436904-3.356472$

C $0.0525683 .190210-1.718426$

H -0.869276 2.853282 -2.214299

H $-0.2040303 .316915-0.653322$

Cl $1.825505-2.1717833 .194019$

C $2.730418-0.8435682 .465879$

C $3.598392-1.1051341 .367160$

C 2.8509670 .3754363 .184417

C $4.620311-0.1730661 .074010$

H $3.615110-2.0975680 .912285$

C 3.8493911 .2783772 .845920

H 2.1862720 .5622624 .029690

C 4.7530420 .9951541 .807666

H $5.318246-0.3992060 .264338$

H 3.9348282 .2114103 .407546

H 5.5515371 .7015911 .572335

TS (oxidative addition) 


$$
E=-2755.33117846
$$

Pd $1.4002610 .174919-1.328903$

P - 2.198878 0.0642090.080481

P 0.7421100 .4092960 .857807

C - 0.9851450 .3403341 .272945

C - 1.4262300 .7615532 .664454

$\mathrm{H}-0.9216631 .6849922 .999016$

$\mathrm{H}-1.2263260 .0011763 .442081$

$\mathrm{H}-2.5025090 .9848632 .727240$

C $-3.727589-0.5560990 .919878$

$\mathrm{H}-4.0368350 .3022911 .543107$

C $-4.890684-0.864310-0.026108$

$\mathrm{H}-4.624065-1.723865-0.664511$

H -5.085657 -0.019462 -0.704350

C $-6.154179-1.1979080 .763415$

H $-6.478384-0.2975571 .316288$

H $-6.974162-1.4477230 .070826$

C $-5.921658-2.3372391 .749208$

H -5.717443 -3.265929 1.185889

H $-6.834128-2.5266012 .337275$

C $-4.741545-2.0450662 .669300$

$\mathrm{H}-4.557465-2.8985203 .341543$

$\mathrm{H}-4.986317-1.1852973 .319412$

C -3.479311-1.728921 1.873557

$\mathrm{H}-2.637517-1.5068812 .543178$

H -3.187333 -2.618840 1.295739

C $-1.664820-1.091501-1.246380$

$\mathrm{H}-0.620813-0.721056-1.418959$

C $-2.398095-1.033310-2.588629$

H -2.516969 $-0.001473-2.944887$

$\mathrm{H}-3.415633-1.448646-2.483935$

C $-1.622748-1.839223-3.629938$

$\mathrm{H}-2.150292-1.808382-4.597036$

H $-0.642749-1.354574-3.788792$

C - $1.404409-3.282239-3.189714$

H -2.379597 -3.801946 -3.153915

H -0.794319 -3.819935 -3.933535

C $-0.753616-3.351778-1.813475$

H $0.270165-2.941621-1.867085$

H $-0.659372-4.398301-1.480759$

C - $1.538824-2.543576-0.787499$

$\mathrm{H}-2.545655-2.984727-0.678417$

$\mathrm{H}-1.055178-2.5923300 .195781$

C $-2.7928851 .576575-0.838480$

H -3.590250 1.259464 -1.534599

C -3.371929 2.6042140.138396

$\mathrm{H}-4.2556622 .1956540 .654100$

H -2.618595 2.8100330 .919176

C -3.749537 $3.909652-0.554946$

$\mathrm{H}-4.1279484 .6294690 .189020$

$\mathrm{H}-4.5829393 .719694-1.255648$

C - $2.5744724 .498494-1.324118$

$\mathrm{H}-2.8756445 .426971-1.835648$

$\mathrm{H}-1.7763274 .777243-0.612577$

C -2.024568 $3.489020-2.322923$

$\mathrm{H}-1.1478583 .900506-2.848737$ 
H -2.7879013.290900 -3.097751

C -1.638670 2.181554-1.641190

$\mathrm{H}-0.7969012 .358360-0.956081$

$\mathrm{H}-1.2565001 .468110-2.386276$

C 1.3949062 .0015611 .589726

H 1.1875691 .9745802 .675011

C 2.9058052 .1292161 .393275

H 3.1129692 .1029480 .308787

H 3.4361531 .2650061 .822148

C 3.4449863 .4242311 .993526

H 3.3179903 .3963803 .091371

H 4.5294943 .5021281 .810126

C 2.7225284 .6453731 .436146

H 2.9494324 .7370510 .358288

H 3.0953915 .5670571 .912262

C 1.2138754 .5211291 .615809

H 0.9713024 .5449142 .693976

H 0.6988475 .3849471 .163048

C 0.6885973 .2232991 .012032

$\mathrm{H}-0.3983193 .1363391 .163360$

H $0.8530083 .235961-0.080863$

C $1.683970-0.8536011 .880413$

H $2.705496-0.8036621 .458949$

C $1.149850-2.2545761 .601412$

H 0.098614 -2.301009 1.939648

H $1.144314-2.4306820 .514010$

C $1.965025-3.3329622 .306208$

H $2.981039-3.3497991 .874497$

H $1.525687-4.3260182 .112985$

C $2.064205-3.0739773 .804314$

H $2.694114-3.8380014 .289082$

H $1.059854-3.1647304 .257454$

C $2.609307-1.6777904 .078763$

H $2.652062-1.4849745 .163609$

H $3.649158-1.6156443 .709030$

C $1.772426-0.6042363 .387159$

H 2.2007430 .3875493 .598046

H $0.757865-0.6039393 .819554$

Cl $2.527083-0.159734-3.444876$

C $3.303139-0.770793-1.848351$

C $4.347630-0.001614-1.304398$

C $3.237165-2.153574-1.602293$

C $5.218069-0.593109-0.396052$

H $4.4401341 .051798-1.571150$

C $4.126195-2.721763-0.695973$

H $2.479512-2.758157-2.102129$

C $5.109994-1.949047-0.075581$

H 5.9997000 .0178250 .062715

H $4.049321-3.790005-0.478395$

H $5.803807-2.4052610 .633186$

$$
\text { L1-Pd(Ar)-Cl }
$$

$E=-2755.38377435$

Pd $1.009919-0.2420291 .381453$

P $-2.068425-0.234073-0.301295$ 
P $0.8705070 .530046-0.765779$

C -0.815644 $0.425465-1.294662$

C -1.153278 $0.680617-2.757991$

$\mathrm{H}-0.2401650 .882216-3.333699$

$\mathrm{H}-1.8039331 .559335-2.920978$

$\mathrm{H}-1.633115-0.173654-3.268413$

C -3.661310 0.355264-1.033256

$\mathrm{H}-3.5135510 .101404-2.098386$

C -4.949064 -0.344408 -0.592640

H -5.159041 -0.1136480 .464625$

H -4.852941 -1.437958 -0.662185

C -6.121343 $0.118973-1.455999$

H -5.956579 -0.224920 -2.493276

H -7.050009 -0.365374 -1.114022

C -6.277365 $1.635528-1.448317$

$\mathrm{H}-6.5687701 .963802-0.434131$

H -7.097929 $1.938099-2.118387$

C $-4.9797122 .333178-1.840931$

H -5.094863 $3.426877-1.774365$

H -4.741803 2.108466 -2.896532

C $-3.8243181 .876029-0.957918$

$\mathrm{H}-2.8837402 .378223-1.230300$

$\mathrm{H}-4.0416082 .1636600 .083430$

C -1.934308 0.1951601.489151

$\mathrm{H}-0.986246-0.3014921 .817867$

C -3.000685 -0.443572 2.388554

$\mathrm{H}-3.104162-1.5200582 .189711$

H -3.977240 0.018977 2.162899

C -2.652047 -0.2227503 .859468$

$\mathrm{H}-3.439596-0.6633954 .491708$

$\mathrm{H}-1.715093-0.7608564 .082158$

C -2.466956 1.254467 4.186475

$\mathrm{H}-3.4345361 .7806914 .083031$

$\mathrm{H}-2.1588651 .3701595 .237568$

C -1.441559 1.899822 3.261444

$\mathrm{H}-0.4483401 .4567043 .451473$

$\mathrm{H}-1.3576762 .9792023 .466562$

C -1.8114001.6853931.798219

$\mathrm{H}-2.7778192 .1738901 .595209$

$\mathrm{H}-1.0759842 .1481491 .132115$

C -2.240543 -2.087530 -0.290034

H -3.197329 -2.313920 0.213087

C -2.314982 -2.606593 -1.729747

$\mathrm{H}-3.185100-2.175989-2.251857$

$\mathrm{H}-1.421938-2.258676-2.275834$

C $-2.380736-4.129223-1.788404$

$\mathrm{H}-2.394277-4.459140-2.839769$

H -3.333058 $-4.470008-1.342928$

C -1.219722 -4.767145 -1.036825

$\mathrm{H}-1.295710-5.865695-1.073693$

H -0.271103 -4.503182 -1.538094

C -1.180990 -4.283239 0.406341

$\mathrm{H}-0.321677-4.7153000 .942832$

$\mathrm{H}-2.085640-4.6399550 .931868$

C -1.114977 -2.762369 0.498756

H -0.140706 -2.414402 0.116381 
$\mathrm{H}-1.137168-2.4657521 .557666$

C $1.888035-0.396446-2.034475$

H $1.461243-0.095930-3.009459$

C $3.381487-0.057495-2.068441$

H $3.834375-0.261454-1.087390$

H $3.5330681 .013808-2.266608$

C $4.110093-0.872711-3.133945$

H $3.746113-0.579074-4.136152$

H $5.184892-0.630737-3.109400$

C $3.895802-2.368873-2.943997$

H $4.338510-2.676129-1.979614$

H $4.420848-2.938148-3.728210$

C $2.409768-2.703463-2.942870$

H $1.985618-2.480111-3.939128$

H $2.252325-3.781620-2.772913$

C $1.662690-1.899679-1.885586$

H $0.585484-2.112035-1.936978$

H $1.996912-2.211807-0.881886$

C $1.5349072 .258599-0.944126$

H $2.6101952 .123030-0.730308$

C 0.9802763 .2128640 .108748

$\mathrm{H}-0.0975133 .360360-0.081308$

H 1.0658922 .7639501 .112368

C 1.6868064 .5636620 .059526

H 2.7483334 .4247610 .333950

H 1.2579045 .2431630 .813922

C $1.6013275 .185868-1.329571$

H $2.1590936 .135797-1.362918$

H $0.5470385 .437018-1.546882$

C $2.1137214 .228634-2.399591$

H $1.9945674 .672147-3.401705$

H $3.1977764 .067656-2.257595$

C $1.3996032 .880562-2.334843$

H $1.7971132 .209578-3.112796$

H $0.3293363 .025970-2.559343$

Cl $0.957886-1.1735533 .530069$

C $2.966110-0.2441761 .307665$

C $3.631249-1.4705501 .225315$

C 3.7015810 .9380061 .406314

C $5.025652-1.5042931 .183215$

H $3.065000-2.4034301 .200213$

C 5.0976970 .8954821 .368427

H 3.1964841 .8999631 .513161

C $5.763663-0.3219081 .241059$

H $5.537474-2.4677231 .110613$

H 5.6653921 .8272011 .439717

H $6.855104-0.3514441 .204340$

$$
\text { L1-Pd(Ar)-Cl(HNMe } \left.{ }_{2}\right)
$$

$E=-2429.70978839$

Pd $1.195010-0.3096631 .212721$

P -2.167819 -0.292086 -0.300582

P $0.7238350 .544357-0.897832$

C $-0.9861650 .362078-1.372925$

C -1.328693 0.397629-2.854791 
H -0.922902 -0.457257-3.428955

$\mathrm{H}-0.9508161 .309108-3.344451$

$\mathrm{H}-2.4127500 .389455-3.041212$

C -3.849113 0.186649 -0.910798

$\mathrm{H}-3.917881-0.347733-1.875579$

C -5.013178 -0.307835 -0.048642

$\mathrm{H}-4.9968910 .2135360 .923562$

$\mathrm{H}-4.918894-1.3823960 .170388$

C -6.345934 -0.035514 -0.742402

H -6.401429 -0.646387-1.661688

$\mathrm{H}-7.177086-0.367643-0.099666$

C -6.506719 1.436474-1.104924

$\mathrm{H}-6.5773072 .031163-0.176086$

$\mathrm{H}-7.4530811 .595622-1.646360$

C -5.326467 $1.940976-1.928162$

H -5.434932 $3.017520-2.136197$

H -5.319164 1.434451-2.910626

C -4.002262 1.679824-1.217990

H -3.151559 2.039114 -1.813899

$\mathrm{H}-3.9817812 .252657-0.278820$

C - 1.9459820 .2524561 .447633

$\mathrm{H}-0.8411660 .1748421 .583400$

C -2.582518 -0.6102472 .541262$

$\mathrm{H}-2.353943-1.6746932 .401162$

$\mathrm{H}-3.680796-0.5121142 .495748$

C -2.067119 -0.169176 3.910159

$\mathrm{H}-2.547368-0.7730614 .696944$

H -0.987230 -0.395995 3.942918

C -2.301218 1.314930 4.165882

$\mathrm{H}-3.3866351 .5075884 .254948$

$\mathrm{H}-1.8545741 .6083565 .129495$

C -1.739627 2.172793 3.037873

$\mathrm{H}-0.6384072 .0914793 .021274$

$\mathrm{H}-1.9751643 .2364713 .203766$

C -2.283939 1.7235201 .687404

$\mathrm{H}-3.3811991 .8463911 .684681$

$\mathrm{H}-1.8882352 .3442900 .873381$

C -2.257524 -2.152743 -0.200662

$\mathrm{H}-3.087932-2.4132260 .479959$

C -2.547757 -2.749365 -1.581539

H -3.522619 -2.402435 -1.959498

$\mathrm{H}-1.787805-2.382181-2.292765$

C $-2.526090-4.274795-1.561364$

$\mathrm{H}-2.705732-4.662014-2.577386$

H -3.361701 -4.639967 -0.937012

C $-1.214156-4.812178-1.004851$

$\mathrm{H}-1.231005-5.913547-0.976021$

$\mathrm{H}-0.387957-4.529304-1.682006$

C $-0.942728-4.2432190 .381226$

H $0.026983-4.5987930 .765136$

$\mathrm{H}-1.710240-4.6132701 .085337$

C - $0.955571-2.7187380 .372176$

$\mathrm{H}-0.113181-2.352257-0.235804$

$\mathrm{H}-0.771744-2.3405811 .389590$

C $1.684558-0.212539-2.311698$

H $1.1926320 .138552-3.236416$ 
C $3.1543340 .210040-2.390303$

H $3.662237-0.020656-1.442212$

H $3.2340451 .298249-2.531219$

C $3.878555-0.497313-3.532690$

H $3.456526-0.164900-4.499396$

H $4.939302-0.198453-3.535946$

C $3.751920-2.011824-3.429595$

H $4.248789-2.352271-2.503307$

H $4.274325-2.502718-4.266954$

C $2.286810-2.427204-3.395004$

H $1.811281-2.163226-4.357531$

H $2.192815-3.521172-3.291827$

C $1.543501-1.733755-2.259702$

H $0.476338-2.004486-2.281581$

H $1.935957-2.089018-1.290569$

C $1.2487762 .327191-0.995052$

H $2.3472882 .259911-0.893484$

C 0.7368953 .1309530 .195851

H -0.361992 3.195658 0.129081

H 0.9637242 .5987561 .135242

C 1.3174744 .5404460 .208674

H 2.4111854 .4794040 .356249

H 0.9193675 .1087141 .065493

C $1.0238055 .270500-1.097558$

H $1.4874546 .270513-1.095320$

H $-0.0664495 .433743-1.179878$

C $1.4974004 .466238-2.303196$

H $1.2296044 .983393-3.239387$

H $2.6006554 .403158-2.287896$

C $0.9210813 .052067-2.298668$

H $1.2963492 .490576-3.169279$

H $-0.1770863 .104304-2.403529$

C $3.150470-0.1513541 .037472$

C $3.921566-1.2946800 .791746$

C 3.8028891 .0800611 .172953

C $5.306268-1.2017800 .640928$

H $3.441295-2.2728870 .704814$

C 5.1888731 .1703811 .025730

H 3.2304861 .9858711 .387938

C 5.9460950 .0329260 .748407

H $5.888431-2.1049430 .436534$

H 5.6791512 .1427451 .128063

H 7.0293980 .1064960 .626737

N $1.272147-1.0333353 .069120$

C $1.727604-2.3816883 .252358$

H $1.321190-3.0470992 .473583$

H $2.836336-2.4832543 .234771$

H $1.393935-2.7831644 .232974$

C $1.786544-0.1762134 .102008$

H $2.897877-0.1351064 .128974$

H 1.4220320 .8558033 .975703

H $1.457939-0.5217345 .105739$

TS (reductive elimination)

$E=-2429.68971575$ 
Pd $1.227937-0.1198921 .176189$ P -2.200962 -0.410414 -0.290041 P $0.5635240 .785553-0.837246$ C - $1.1311350 .486584-1.300189$ C -1.544684 $0.702092-2.747088$ $\mathrm{H}-1.3904381 .741356-3.080051$ $\mathrm{H}-2.6091140 .485672-2.923284$ H -0.992921 $0.061717-3.461199$ C -3.945952 $0.016437-0.736051$ H -4.051031 -0.383953-1.760658 C -5.011123 -0.6701300 .121829$ $\mathrm{H}-4.947787-0.2908091 .155953$ $\mathrm{H}-4.839912-1.7562770 .175146$ C -6.407379 $-0.390011-0.428740$ $\mathrm{H}-6.501777-0.863215-1.422947$ H -7.165961 -0.8655160 .213759$ C -6.675416 $1.105426-0.554572$ $\mathrm{H}-6.7013341 .5533970 .455441$ H -7.669894 $1.280099-0.995782$ C -5.594726 $1.798205-1.377523$ $\mathrm{H}-5.7770972 .884188-1.415569$ H -5.641489 $1.439263-2.421898$ C -4.203682 $1.524252-0.815371$ $\mathrm{H}-3.4266972 .013600-1.419191$ $\mathrm{H}-4.1299421 .9626840 .191923$ C -1.910462 -0.124072 1.505654 H -0.794739 -0.2030641 .540541$ C -2.432270 -1.164144 2.500208 H -2.219264 -2.189472 2.170259 H -3.529475 -1.082750 2.585998 C $-1.787919-0.9245213 .865300$ H -2.159937 -1.666652 4.589895 H -0.699032 -1.085088 3.768179 C -2.042399 0.4901334 .373750 $\mathrm{H}-3.1207150 .6129644 .585356$ H -1.518630 0.650464 5.329922 C - 1.6121211 .5352733 .350978 H -0.5142271 .5001393 .228762$ $\mathrm{H}-1.8608182 .5492443 .703389$ C -2.2564231.2808231.992860 $\mathrm{H}-3.3530141 .3729272 .087260$ $\mathrm{H}-1.9301752 .0290041 .260497$ C -2.143021 -2.268166 -0.465887 $\mathrm{H}-2.925221-2.6969250 .186554$ C $-2.437657-2.671872-1.914143$ $\mathrm{H}-3.453395-2.360016-2.205382$ $\mathrm{H}-1.741759-2.132726-2.579752$ C - $2.284097-4.173620-2.134121$ $\mathrm{H}-2.472350-4.415012-3.192808$ H -3.057136 -4.706655 -1.551148 C - $0.907481-4.665819-1.707108$ H -0.825534 -5.754870 -1.853628 H - $0.138845-4.203897-2.352610$ C -0.630257 -4.297438 -0.255782 H $0.382494-4.6172590 .039343$ H - $1.332523-4.8449880 .399388$ 
C $-0.777056-2.798436-0.020229$

H $0.005675-2.260373-0.575145$

$\mathrm{H}-0.589796-2.5675461 .038886$

C $1.5849260 .245947-2.306110$

H $1.1392270 .726543-3.196668$

C $3.0483780 .684363-2.211827$

H $3.4861700 .295685-1.279168$

H $3.1228511 .780872-2.157316$

C $3.8649050 .184746-3.400737$

H $3.5080450 .677025-4.324645$

H $4.9184090 .482442-3.273856$

C $3.757661-1.325746-3.566612$

H $4.210427-1.814032-2.685819$

H $4.332435-1.660806-4.445673$

C $2.300882-1.756140-3.682796$

H $1.870300-1.344117-4.614098$

H $2.224657-2.853574-3.764568$

C $1.485300-1.266095-2.492138$

H $0.429732-1.557987-2.603867$

H $1.853395-1.753403-1.572319$

C $0.9235622 .620499-0.816079$

H $2.0080032 .640427-0.597011$

C 0.2215253 .3150190 .345723

H -0.867398 3.2828850.163807

H 0.4081232 .7574731 .278963

C 0.6660314 .7671130 .486679

H 1.7360524 .7900970 .762160

H 0.1221205 .2528651 .313818

C $0.4634315 .541640-0.810890$

H $0.8369946 .573697-0.708177$

H -0.619792 5.622358 -1.016436

C $1.1392444 .842575-1.985154$

H $0.9424735 .388276-2.922800$

H $2.2347044 .860258-1.839368$

C $0.6786803 .392278-2.112065$

H $1.1833432 .910264-2.963948$

H -0.401925 $3.378100-2.335428$

C $3.214730-0.4588631 .247181$

C $3.732739-1.6207450 .645640$

C 4.0695460 .6486841 .399341

C $5.044940-1.6539000 .177075$

H $3.097562-2.4992640 .514529$

C 5.3788100 .6046130 .923956

H 3.6987281 .5665531 .860290

C $5.878891-0.5423830 .305290$

H $5.416104-2.564387-0.302272$

H 6.0142071 .4881121 .033235

H $6.905043-0.571247-0.066986$

N $2.053550-0.8647462 .866006$

C $2.200583-2.2648523 .127062$

H $1.751153-2.8655552 .323610$

H $3.262110-2.5788643 .233979$

H $1.677441-2.5365394 .065687$

C $2.554460-0.0356463 .921538$

H $3.641219-0.1794234 .108099$

H 2.3886531 .0274423 .698445 
H $2.021040-0.2552904 .867872$

L1-Pd-( $\left.\mathrm{ArNMe}_{2}\right)$

$E=-2429.74129086$

Pd 1.3550620 .5157651 .010136

P -2.111205 -0.711973 -0.220224

P $0.2655471 .106621-0.850751$

C -1.286434 0.349074 -1.287899

C -1.747593 0.469310 -2.726786

$\mathrm{H}-1.6719571 .510677-3.088470$

$\mathrm{H}-2.8001860 .179482-2.874006$

$\mathrm{H}-1.162262-0.143217-3.440246$

C -3.916234 -0.677434 -0.638466

H -3.931545 -1.036693 -1.683920

C $-4.812333-1.6202550 .166556$

$\mathrm{H}-4.836404-1.2941591 .219875$

$\mathrm{H}-4.414749-2.6465850 .164970$

C -6.234652 -1.614824 -0.389245

$\mathrm{H}-6.222073-2.039305-1.409597$

H -6.875898 -2.277851 0.214015

C -6.818026 -0.207092 -0.436746

$\mathrm{H}-6.9414860 .1669450 .595954$

$\mathrm{H}-7.826059-0.224938-0.881762$

C -5.909261 $0.746260-1.204911$

$\mathrm{H}-6.3187661 .769100-1.184543$

$\mathrm{H}-5.8776050 .446070-2.268248$

C -4.492148 $0.740993-0.642512$

$\mathrm{H}-3.8349101 .415869-1.208819$

$\mathrm{H}-4.5108511 .1283110 .389089$

C - $1.796499-0.3234751 .545544$

$\mathrm{H}-0.672086-0.3079021 .533271$

C -2.264741 -1.349947 2.578080

$\mathrm{H}-2.006845-2.3776252 .284507$

$\mathrm{H}-3.364375-1.3192522 .666430$

C -1.648709-1.035566 3.939683

H -2.007083 -1.758235 4.691102

H - $0.556177-1.1719603 .868622$

C - 1.9494930 .3909164 .387711

$\mathrm{H}-3.0315680 .4801644 .596811$

$\mathrm{H}-1.4347180 .6081305 .338432$

C - 1.5625831 .4093523 .321551

$\mathrm{H}-0.4657231 .4095963 .171831$

$\mathrm{H}-1.8418892 .4266713 .640678$

C -2.2060851.0810211.979999

$\mathrm{H}-3.3047941 .1470142 .069773$

$\mathrm{H}-1.8977361 .8079131 .218611$

C -1.675669 -2.525335 -0.353761

$\mathrm{H}-2.380204-3.0998820 .274534$

C - $1.832644-2.982238-1.807625$

$\mathrm{H}-2.883037-2.890944-2.128513$

$\mathrm{H}-1.250492-2.303314-2.453186$

C - $1.352571-4.413678-2.023025$

$\mathrm{H}-1.447353-4.681274-3.087969$

H -2.009606 $-5.107730-1.467647$ 
C $0.082840-4.597221-1.548018$

H $0.407910-5.639732-1.696749$

H $0.754032-3.967378-2.159070$

C $0.216393-4.198166-0.084910$

H $1.260897-4.3067160 .250104$

H -0.381644 -4.892017 0.534544

C -0.252330 -2.767586 0.156570

H $0.431949-2.056959-0.334222$

$\mathrm{H}-0.188010-2.5347551 .230111$

C $1.3838770 .709564-2.308334$

H $0.8541991 .050729-3.218154$

C $2.7284491 .433403-2.240660$

H $3.2140661 .168288-1.282752$

H $2.5827212 .524441-2.229487$

C $3.6387071 .056666-3.407206$

H $3.1926911 .425009-4.349605$

H $4.6101991 .568824-3.304563$

C $3.841023-0.450386-3.504123$

H $4.382538-0.798698-2.607433$

H $4.470583-0.701344-4.374085$

C $2.503351-1.175692-3.580508$

H $1.998625-0.917251-4.529829$

H $2.658127-2.268050-3.597995$

C $1.604804-0.796197-2.409588$

H $0.633990-1.308690-2.484799$

H 2.067679 -1.137257 -1.465039

C $0.0571632 .965194-1.134834$

H $0.3303153 .145842-2.192547$

C $1.0166283 .764237-0.248487$

H 0.7577363 .5570080 .805981

H $2.0491103 .402735-0.366478$

C $0.9345645 .261744-0.528690$

H $1.2897225 .457996-1.557239$

H 1.6141505 .8125610 .143009

C $-0.4913215 .781254-0.383629$

H -0.543539 $6.851579-0.643540$

H -0.7957405 .7032100 .676276$

C - $1.4632614 .974028-1.237217$

$\mathrm{H}-2.4963945 .326857-1.080171$

H -1.236782 5.142799-2.306032

C - $1.3654973 .481368-0.935344$

H -2.077213 2.910707 -1.548720

$\mathrm{H}-1.6657363 .3126990 .112773$

C $3.663370-0.7867091 .597213$

C $3.314625-2.0908631 .196976$

C $4.824300-0.2190521 .047784$

C $4.073943-2.7743770 .256941$

H $2.416975-2.5632141 .592971$

$\mathrm{ArCl}=p$-Chlorotoluene

L1-Pd(ArCl) 
$E=-2794.63310792$

Pd $1.5571480 .296527-0.765007$

P - 2.195387 0.091356 0.021028

P $0.575963-0.1163851 .285007$

C - $1.197749-0.1220441 .410677$

C - 1.8435830 .0307212 .777810

$\mathrm{H}-1.5485580 .9619793 .297811$

$\mathrm{H}-1.594939-0.7970953 .462163$

$\mathrm{H}-2.9426330 .0615972 .730454$

C $-3.865360-0.6257420 .383382$

$\mathrm{H}-4.2562520 .0405561 .173768$

C $-4.863087-0.569559-0.775393$

H -4.520950 -1.236366 -1.584903

H -4.918619 $0.442621-1.204880$

C $-6.248111-1.016753-0.313773$

H $-6.632050-0.2894760 .424493$

H -6.950915 -0.996972 -1.162410

C $-6.211683-2.4042100 .316954$

H -5.944833 -3.145498 -0.458170

H -7.212693 -2.685353 0.682019

C $-5.189332-2.4765451 .445957$

H $-5.139846-3.4985591 .854978$

H -5.514373 -1.825239 2.277827

C -3.806480 -2.036411 0.976957

H -3.077115 -2.074964 1.797895

H -3.443460 -2.740750 0.212292

C -1.482803 - $0.647674-1.505914$

$\mathrm{H}-0.427126-0.274462-1.441322$

C - $2.052032-0.178313-2.847645$

$\mathrm{H}-2.1307420 .915696-2.899270$

H -3.073253 -0.574123 -2.980046

C -1.163383 -0.672661 -3.987143

$\mathrm{H}-1.588094-0.356717-4.953790$

H $-0.183701-0.172799-3.901690$

C - $0.978941-2.186026-3.959866$

$\mathrm{H}-1.943317-2.671169-4.198654$

H - $0.275082-2.497902-4.749242$

C - $0.502050-2.673716-2.596422$

H $0.520832-2.304819-2.403737$

H -0.450636 -3.774534-2.577089

C - $1.411871-2.173093-1.481555$

$\mathrm{H}-2.425177-2.588619-1.625979$

$\mathrm{H}-1.056991-2.520713-0.504122$

C $-2.5705551 .852889-0.465068$

$\mathrm{H}-3.2902921 .828671-1.303460$

C -3.209540 2.6042430 .707005

$\mathrm{H}-4.1745372 .1483000 .980997$

H -2.555016 2.5040331 .589838

C -3.4147314.0841920.398830

$\mathrm{H}-3.8444324 .5906381 .278429$

$\mathrm{H}-4.1577974 .186469-0.412999$

C - $2.1145124 .753956-0.026747$

H -2.288544 $5.815983-0.264094$

$\mathrm{H}-1.4014334 .7342520 .817237$

C - $1.5018724 .030371-1.218188$

H - $0.5405544 .487792-1.502458$ 
H -2.169510 4.141909-2.092443

C -1.288000 2.549416 -0.928817

H $-0.5230292 .432267-0.146761$

H -0.865483 2.058755 -1.816833

C 1.1643841 .1199402 .563512

H 0.7050770 .8453793 .530949

C 2.6845521 .1028202 .732643

H 3.1452141 .2692861 .741434

H 3.0285300 .1132113 .069966

C 3.1632042 .1657833 .718346

H 2.7908301 .9157174 .728696

H 4.2638382 .1487783 .782623

C 2.6725793 .5575673 .339173

H 3.1320053 .8550412 .378779

H 3.0007944 .2999424 .085040

C 1.1564093 .5763283 .190915

H 0.6908663 .3818204 .174473

H 0.8097374 .5745972 .875188

C 0.6895682 .5226982 .194110

H -0.4070712 .5339132 .103975$

H 1.0860902 .7659811 .191083

C $1.289567-1.6989081 .990416$

H $2.375808-1.4925661 .960804$

C $1.048498-2.8852211 .063395$

H -0.032659 -3.110766 1.056234

H $1.329495-2.6111030 .032670$

C $1.814609-4.1211161 .523048$

H 2.899045 -3.922322 1.439924

H $1.603301-4.9716400 .853661$

C $1.476505-4.4827922 .965297$

H $2.075474-5.3458113 .299847$

H $0.418900-4.8004383 .016856$

C $1.682209-3.2954013 .899513$

H $1.384247-3.5594214 .927842$

H $2.758698-3.0477963 .942159$

C $0.909220-2.0671943 .423155$

H $1.082110-1.2237804 .110551$

H -0.171709 -2.286754 3.458365

Cl $1.7949741 .952332-3.421376$

C $2.6733640 .708256-2.531889$

C $3.5351771 .076289-1.462041$

C $2.785825-0.587510-3.100634$

C $4.5167760 .153371-1.031265$

H $3.5838362 .116388-1.133486$

C $3.745400-1.467911-2.629175$

H $2.139931-0.859975-3.937017$

C $4.638217-1.110115-1.595472$

C $5.645784-2.099429-1.089967$

H $5.157714-2.877223-0.477445$

H $6.160490-2.614304-1.915760$

H $6.407776-1.615214-0.462921$

H $5.2004970 .459268-0.234706$

H $3.813253-2.463063-3.077191$

TS (oxidative addition) 
$E=-2794.61621241$

$\mathrm{Pd}-1.2044490 .4336821 .393727$

P $2.313317-0.092685-0.125994$

P $-0.6066670 .496064-0.823367$

C $1.0892150 .242914-1.292019$

C $1.5131710 .551534-2.717663$

H $1.0801971 .499090-3.083596$

H $1.216570-0.225577-3.446581$

H $2.6019160 .672564-2.827969$

C $3.750387-0.886995-0.981301$

H $4.116494-0.088254-1.651242$

C $4.909864-1.260860-0.054440$

H $4.585768-2.0633410 .630122$

H $5.202632-0.4091710 .578625$

C $6.112256-1.744220-0.861650$

H $6.500340-0.902577-1.463674$

H $6.926950-2.039217-0.180704$

C $5.745520-2.898197-1.787735$

H $5.474246-3.778734-1.177355$

H $6.618002-3.196936-2.390903$

C $4.569403-2.536733-2.688132$

H $4.287223-3.397016-3.316195$

H $4.871997-1.731698-3.382480$

C $3.366504-2.071432-1.873905$

H $2.528378-1.801289-2.530222$

H $3.012614-2.905058-1.248964$

C $1.722506-1.1381981 .266952$

H $0.717767-0.6755481 .447272$

C $2.495714-1.0798462 .586569$

H $2.706194-0.0461272 .891065$

H $3.473615-1.5801222 .475930$

C $1.688495-1.7728703 .683296$

H $2.243861-1.7410694 .634643$

H $0.755010-1.2039323 .843192$

C $1.344660-3.2120243 .316883$

H 2.274508 -3.809087 3.281189

H $0.715612-3.6654294 .100010$

C $0.651217-3.2909991 .962193$

H - $0.335591-2.7998212 .022250$

H $0.466293-4.3405761 .681129$

C $1.467043-2.5944630 .879849$

H $2.431815-3.1199300 .765224$

H $0.951830-2.647698-0.087206$

C 3.0744611 .3971680 .702155

H 3.8606281 .0390011 .391004

C $3.7146512 .318826-0.340182$

H $4.5399251 .805232-0.858718$

H $2.9588272 .556711-1.109476$

C 4.2337743 .6143540 .276042

H $4.6523114 .259519-0.513526$

H 5.0688313 .3801470 .961216

C 3.1446174 .3467021 .048363

H 3.5475955 .2653301 .504609

H 2.352747 4.666218 0.347035

C 2.5365343 .4411252 .110912

H 1.7196163 .9573722 .640568 
H 3.3030613 .2076372 .872614

C 2.0082342 .1461081 .505161

H 1.1663272 .3712560 .834588

H 1.5834951 .5086052 .294895

C - $1.1365142 .106542-1.612592$

H - $0.9833222 .005593-2.702462$

C -2.617828 2.388281-1.359563

$\mathrm{H}-2.7732262 .438574-0.267232$

H -3.246423 $1.557052-1.714581$

C -3.062370 3.695807-2.008046

$\mathrm{H}-2.9912773 .599097-3.107044$

H -4.124799 $3.886587-1.782914$

C -2.203186 4.869198 -1.551163

$\mathrm{H}-2.3697515 .038290-0.471680$

$\mathrm{H}-2.5099475 .796398-2.062441$

C - $0.7227274 .591655-1.786299$

$\mathrm{H}-0.5296594 .535791-2.873249$

$\mathrm{H}-0.1087835 .424676-1.404786$

C - $0.2930453 .282718-1.132749$

H $0.7729783 .084551-1.323152$

$\mathrm{H}-0.4055683 .368407-0.036673$

C -1.697758 $-0.720510-1.750879$

H -2.690685 -0.571795-1.286489

C - $1.262188-2.148721-1.440026$

$\mathrm{H}-0.233520-2.289884-1.818933$

$\mathrm{H}-1.222327-2.285779-0.347908$

C -2.188549 -3.184710 -2.066620

H -3.181028 -3.109284-1.589455

$\mathrm{H}-1.816928-4.201290-1.854777$

C $-2.338652-2.972138-3.567710$

$\mathrm{H}-3.047795-3.701053-3.993609$

$\mathrm{H}-1.367096-3.157446-4.061585$

C $-2.786649-1.548091-3.872338$

$\mathrm{H}-2.866171-1.391143-4.960829$

H -3.800606 -1.390727 -3.460379

C - $1.838537-0.519356-3.260997$

$\mathrm{H}-2.2000880 .494293-3.491568$

H -0.848877 -0.613248 -3.738625

$\mathrm{Cl}-2.3236340 .3037473 .524522$

C -3.147973 -0.360240 1.946489

C -4.1553510 .4291491 .365042$

C -3.186335 -1.756171 1.800939

C $-5.074368-0.1672000 .511260$

H -4.1828511 .5023841 .556785$

C $-4.125800-2.3245140 .947476$

$\mathrm{H}-2.468135-2.3791062 .335362$

C $-5.072278-1.5486440 .266569$

C $-6.037366-2.166274-0.701646$

H -5.669846 -2.079965 -1.739417

H -6.184451 -3.236886 -0.498064

H -7.021154 -1.674672 -0.665715

$\mathrm{H}-5.8222860 .4641630 .022441$

H -4.121837 -3.4098360.811719

L1-Pd(Ar)-Cl 
$E=-2794.67148115$

Pd $1.0277540 .544312-1.356871$

P -2.152547 -0.152054 0.141069

P 0.6970660 .6005200 .918072

C - 1.0089400 .3557181 .330414

C -1.479144 0.8027332 .703509

$\mathrm{H}-1.0266001 .7633473 .001707$

$\mathrm{H}-1.2395170 .0845363 .509986$

$\mathrm{H}-2.5651080 .9732982 .751901$

C $-3.728840-0.6126240 .984853$

$\mathrm{H}-4.0294550 .3393181 .459168$

C $-4.855729-1.0120850 .027445$

$\mathrm{H}-4.599654-1.968497-0.459580$

H -4.974085 -0.273040 -0.779375

C $-6.172862-1.1650080 .784306$

$\mathrm{H}-6.478212-0.1758181 .171114$

$\mathrm{H}-6.967182-1.4842410 .090706$

C $-6.052151-2.1447211 .946010$

H -5.873582 -3.159309 1.546090

H -7.001152 -2.194933 2.503469

C $-4.903550-1.7689712 .875320$

H -4.798922 -2.515490 3.678795

H -5.129472 -0.807808 3.372149

C $-3.589842-1.6443882 .110177$

$\mathrm{H}-2.767394-1.3768682 .786394$

$\mathrm{H}-3.333533-2.6246751 .683237$

C - $1.547906-1.528951-0.934808$

$\mathrm{H}-0.483378-1.236499-1.074221$

C $-2.162514-1.648766-2.332650$

$\mathrm{H}-2.149213-0.688866-2.864910$

H -3.219869 -1.956853 -2.253653

C -1.381812 -2.672792 -3.153505

$\mathrm{H}-1.831655-2.763918-4.154883$

$\mathrm{H}-0.361201-2.281412-3.310606$

C - $1.330091-4.033560-2.469760$

$\mathrm{H}-2.348194-4.463749-2.439420$

H -0.716087 -4.734159-3.057981

C -0.796648 -3.927221-1.046048

H $0.269581-3.644648-1.068597$

H $-0.844344-4.905025-0.540006$

C - $1.568896-2.891014-0.237333$

$\mathrm{H}-2.614943-3.230501-0.143841$

$\mathrm{H}-1.162979-2.8109620 .780192$

C $-2.6655221 .188083-1.050451$

H -3.378224 $0.747991-1.769357$

C -3.350646 2.336651 -0.306182

$\mathrm{H}-4.2944931 .9937830 .146841$

H -2.698786 2.6541390.526711

C -3.631341 3.529511-1.216004

H -4.086309 4.342667 -0.627583

$\mathrm{H}-4.3812953 .236995-1.973098$

C - $2.3728604 .014988-1.924105$

$\mathrm{H}-2.6091844 .861620-2.588154$

$\mathrm{H}-1.6558524 .396588-1.175406$

C - $1.7288672 .884023-2.712932$

H -0.790926 3.202425 -3.192769 
H -2.404346 $2.575068-3.530333$

C -1.444078 $1.676714-1.827900$

$\mathrm{H}-0.6644591 .976573-1.089767$

$\mathrm{H}-1.0349100 .876057-2.465565$

C 1.1743632 .2771711 .579426

H 1.0116972 .2340132 .671435

C 2.6458342 .6047621 .329696

H 2.8064702 .6487890 .238861

H 3.3048751 .8083231 .707628

C 3.0240873 .9465551 .949954

H 2.9304913 .8774913 .049188

H 4.0828884 .1714491 .742706

C 2.1315735 .0701191 .434868

H 2.324545 5.216078 0.356651

H 2.3855926 .0226451 .927443

C 0.6559694 .7424281 .634358

H 0.4276344 .7191782 .715503

H 0.0231845 .5342421 .200632

C 0.2971913 .3934021 .020408

H -0.765966 3.160829 1.182439

H $0.4446413 .444306-0.073407$

C $1.775832-0.5507631 .908663$

H $2.761244-0.4836941 .414720$

C $1.273928-1.9826111 .751218$

H $0.272417-2.0522452 .211901$

H $1.150216-2.2206370 .685314$

C $2.220425-2.9817012 .404058$

H $3.183912-2.9612931 .864150$

H $1.823306-4.0045542 .295596$

C $2.444880-2.6498863 .874547$

H $3.172675-3.3456804 .322953$

H $1.497541-2.7927744 .426217$

C $2.910450-1.2091624 .051549$

H $3.023206-0.9687885 .121542$

H $3.911560-1.0937683 .598336$

C $1.956778-0.2155563 .390424$

H 2.3462530 .8050873 .523167

H $0.979062-0.2483143 .901714$

Cl $1.2319920 .490472-3.687065$

C $2.710799-0.449418-1.163572$

C $3.9236700 .184370-0.894620$

C $2.706541-1.833637-1.351677$

C $5.095401-0.564084-0.757372$

H $3.9738491 .268205-0.790062$

C $3.879096-2.572606-1.207227$

H $1.784266-2.347786-1.627045$

C $5.095794-1.954634-0.894439$

C $6.356689-2.758364-0.753457$

H $6.203365-3.641592-0.113947$

H $6.707579-3.125374-1.732268$

H $7.168293-2.160160-0.315137$

H $6.034106-0.045370-0.539698$

H $3.845361-3.657353-1.349765$

L1-Pd(Ar)-Cl(HNMe 2$)$ 
$E=-2468.99385364$

Pd $1.051644-0.3014531 .198507$

P - $2.324004-0.291871-0.281588$

P $0.5613530 .536719-0.913972$

C - $1.1536400 .353528-1.371345$

C - $1.5117100 .378955-2.849643$

$\mathrm{H}-1.112289-0.480152-3.421988$

$\mathrm{H}-1.1385651 .286826-3.349562$

$\mathrm{H}-2.5976860 .369917-3.024656$

C -4.011728 $0.182907-0.877072$

H $-4.091229-0.359146-1.836721$

C -5.166262 -0.3044300 .001867$

$\mathrm{H}-5.1393060 .2248130 .969594$

H -5.069515 -1.377160 0.228653

C -6.506608 $-0.037755-0.679343$

H -6.572215 $-0.656194-1.592893$

H -7.330631 -0.364491-0.024763

C $-6.6713861 .431189-1.052258$

$\mathrm{H}-6.7317132 .033554-0.127650$

$\mathrm{H}-7.6236621 .585943-1.584534$

C -5.500254 $1.928756-1.892584$

H $-5.6110843 .003535-2.108389$

H -5.503732 $1.414053-2.870814$

C -4.168319 $1.673512-1.194817$

$\mathrm{H}-3.3242462 .027851-1.802986$

H -4.137661 2.254135 -0.260748

C -2.083019 0.266855 1.459709

$\mathrm{H}-0.9764100 .1932711 .582811$

C - $2.704472-0.5883212 .567727$

$\mathrm{H}-2.473820-1.6531342 .434010$

H -3.803539 -0.4940542 .533696$

C -2.174991 -0.1342353 .926973$

$\mathrm{H}-2.644891-0.7326304 .724146$

$\mathrm{H}-1.094214-0.3583363 .949472$

C -2.410004 1.351380 4.172811

$\mathrm{H}-3.4948421 .5422684 .272336$

$\mathrm{H}-1.9533881 .6539805 .128890$

C -1.8631012.2010183.031448

H -0.761969 2.1220093.003025

H -2.099258 3.2655323.191036

C -2.4217441.7390961.691156

$\mathrm{H}-3.5192781 .8592911 .700075$

$\mathrm{H}-2.0371092 .3539230 .867281$

C $-2.414421-2.151720-0.165998$

H -3.235990 -2.405858 0.527645

C $-2.723722-2.759059-1.538066$

$\mathrm{H}-3.702981-2.413820-1.906045$

H - $1.972629-2.399002-2.262280$

C -2.704244 -4.284324 -1.505532

H -2.897886 -4.679647 -2.515858

$\mathrm{H}-3.532221-4.642784-0.867263$

C - $1.386086-4.819545-0.961816$

$\mathrm{H}-1.404562-5.920611-0.923530$

H $-0.568369-4.543919-1.652134$

C - $1.095354-4.2394280 .415728$

H -0.121201 -4.593433 0.789728 
H $-1.854088-4.6021321 .133060$

C - $1.105693-2.7150950 .393656$

$\mathrm{H}-0.271273-2.355408-0.229035$

H -0.906993 -2.328299 1.405035

C $1.507042-0.228673-2.333884$

H $1.0043050 .116346-3.255080$

C $2.9756380 .194365-2.432612$

H $3.495032-0.028024-1.488892$

H $3.0523521 .281496-2.583392$

C $3.687694-0.521330-3.577413$

H $3.255122-0.196987-4.542229$

H $4.748166-0.221709-3.594454$

C $3.563395-2.035029-3.460845$

H $4.069562-2.367355-2.536660$

H $4.077676-2.532381-4.299459$

C $2.098907-2.450735-3.408411$

H $1.613644-2.193906-4.368027$

H $2.006476-3.543980-3.296418$

C $1.366756-1.749550-2.270582$

H $0.299393-2.020350-2.280438$

H $1.768590-2.098366-1.303094$

C $1.0871672 .318436-1.026507$

H $2.1869942 .249579-0.942607$

C 0.5964693 .1271140 .169952

H -0.503424 3.1916420.122982

H 0.8403272 .5983321 .107016

C 1.1773014 .5365270 .167107

H 2.2733834 .4758510 .296161

H 0.7940555 .1083121 .028359

C $0.8615145 .261434-1.136823$

H $1.3248426 .261571-1.146343$

H $-0.2300245 .424080-1.201504$

C $1.3150874 .452454-2.346995$

H $1.0319154 .966003-3.280663$

H $2.4184624 .389404-2.349601$

C $0.7389723 .038322-2.327469$

H $1.1004992 .473219-3.201597$

H - $0.3607633 .090005-2.415097$

C $3.005724-0.1619040 .988767$

C $3.765069-1.3103520 .735072$

C 3.6760411 .0613571 .091424

C $5.144153-1.2277100 .543460$

H $3.279322-2.2874530 .667372$

C 5.0574351 .1357900 .902773

H 3.1216851 .9781531 .307328

C $5.817705-0.0025930 .615563$

H $5.709745-2.1401650 .328308$

H 5.5547792 .1083030 .976148

N $1.152466-1.0072243 .060653$

C $1.606713-2.3549373 .251110$

H $1.185033-3.0276162 .486796$

H $2.714700-2.4604303 .215536$

H 1.288677 -2.744568 4.241707

C $1.685283-0.1409584 .076366$

H $2.797055-0.1026634 .085513$

H 1.3216560 .8907513 .944891 
H $1.371465-0.4749745 .088763$

C 7.3060300 .0778810 .429473

H 7.6180761 .0813460 .104735

H $7.839555-0.1420051 .369841$

H $7.658992-0.646647-0.319772$

TS (reductive elimination)

$E=-2468.97349658$

Pd $1.067262-0.0584251 .212359$

P - $2.327261-0.459246-0.314275$

P $0.4028530 .833221-0.808637$

C - $1.2730160 .480053-1.302207$

C - $1.6694930 .688787-2.754858$

$\mathrm{H}-1.5499491 .735315-3.079328$

$\mathrm{H}-2.7214680 .432417-2.951119$

$\mathrm{H}-1.0810270 .074833-3.462817$

C -4.078425 $-0.096943-0.792351$

H -4.149548 -0.496881-1.820026

C -5.132048 -0.8267300 .043965$

H -5.099958 -0.4510271 .080890$

$\mathrm{H}-4.921956-1.9061970 .094905$

C -6.528347 -0.595639-0.528867

$\mathrm{H}-6.588564-1.067230-1.526512$

H -7.279366 -1.102036 0.098756

C -6.849615 $0.889363-0.653145$

$\mathrm{H}-6.9084631 .3315340 .358045$

H -7.842569 1.029110 -1.109974

C -5.781948 $1.624997-1.455532$

$\mathrm{H}-6.0037282 .703653-1.492852$

H -5.798220 1.268838 -2.501790

C -4.391241 1.400451-0.871206

H -3.623298 $1.920630-1.460605$

$\mathrm{H}-4.3501501 .8373270 .138525$

C -2.080564 -0.166840 1.486867

$\mathrm{H}-0.963107-0.2007311 .539441$

C -2.576456 -1.230275 2.469808

$\mathrm{H}-2.315495-2.2449172 .141220$

H $-3.677460-1.1945492 .537027$

C -1.965930 -0.9686023 .846264$

$\mathrm{H}-2.319275-1.7276234 .562698$

H $-0.869858-1.0836883 .767331$

C -2.287108 0.4328524 .353611

$\mathrm{H}-3.3730590 .5107834 .546817$

$\mathrm{H}-1.7869140 .6116385 .319103$

C - -1.8825191 .4979143 .340881$

$\mathrm{H}-0.7821371 .5084473 .238022$

H -2.178925 2.499700 3.691300

C -2.492204 1.2212871.971089

$\mathrm{H}-3.5931481 .2681492 .046769$

$\mathrm{H}-2.1845521 .9847201 .246287$

C -2.198392 $-2.313179-0.493790$

$\mathrm{H}-2.973467-2.7715870 .146761$

C $-2.458255-2.722388-1.947093$

H -3.481069 -2.448153 -2.251030 
$\mathrm{H}-1.774444$-2.154959 -2.601651

C -2.245688 -4.216414 -2.170509

$\mathrm{H}-2.410097-4.460083-3.232656$

H $-3.006360-4.780354-1.600474$

C $-0.857845-4.658765-1.726009$

$\mathrm{H}-0.733102-5.743338-1.875634$

H $-0.097983-4.165495-2.358579$

C $-0.615376-4.286810-0.269357$

H $0.404154-4.5704670 .039263$

$\mathrm{H}-1.306344-4.8628590 .373279$

C - $0.820631-2.795199-0.030128$

H -0.049864 -2.226425 -0.570919

H -0.657818 -2.561492 1.032445

C $1.4648780 .337845-2.264978$

H $1.0210200 .815620-3.157972$

C $2.9132820 .816041-2.139959$

H $3.3455390 .427766-1.204711$

H $2.9556561 .913440-2.070006$

C $3.7641410 .355809-3.320776$

H $3.4102940 .851252-4.244151$

H $4.8068060 .680720-3.171269$

C $3.702037-1.154614-3.509579$

H 4.154368 -1.642553 -2.628442

H $4.299383-1.460553-4.384258$

C $2.259969-1.624296-3.654406$

H $1.832080-1.211993-4.586851$

H 2.216646 -2.722247 -3.751951

C $1.412740-1.174018-2.470072$

H $0.368147-1.495821-2.600604$

H $1.782621-1.661701-1.551466$

C $0.6986482 .679925-0.774099$

H $1.7776132 .737046-0.535389$

C -0.048588 3.342965 0.377895

$\mathrm{H}-1.1321903 .2716630 .176582$

H 0.1420742 .7880531 .311869

C 0.3393684 .8097040 .533098

H 1.4026644 .8709210 .828091

H -0.236967 5.2709281 .352294

C $0.1316955 .582588-0.764625$

H $0.4645136 .627351-0.650729$

H $-0.9498455 .623835-0.989815$

C $0.8545584 .914775-1.928988$

H $0.6553715 .457586-2.867820$

H $1.9457114 .972074-1.762445$

C $0.4501793 .449168-2.070925$

H $0.9883932 .990454-2.915104$

$\mathrm{H}-0.6246833 .396499-2.315203$

C $3.055096-0.3985391 .288405$

C $3.569918-1.5587570 .683144$

C 3.9162220 .7021811 .432398

C $4.873829-1.5889930 .196037$

H $2.935658-2.4387050 .556125$

C 5.2183170 .6554200 .937120

H 3.5565571 .6234481 .895434

C $5.727096-0.4832900 .302459$

H $5.234145-2.500333-0.292334$ 
H 5.8532121 .5415221 .037005

N $1.887533-0.7956912 .907746$

C $2.041354-2.1938913 .174844$

H $1.597245-2.8002862 .372724$

H $3.104316-2.5018063 .285634$

H $1.517491-2.4646564 .113382$

C 2.3894550 .0394903 .957971

H $3.477041-0.1014654 .142546$

H 2.220739 1.101033 3.729808

H $1.858855-0.1768354 .906600$

C $7.132923-0.532235-0.222761$

H $7.179187-1.023813-1.207175$

H $7.5557440 .477293-0.331351$

H $7.800416-1.0961590 .451167$

L1-Pd-( $\left.\mathrm{ArNMe}_{2}\right)$

$E=-2469.02666065$

Pd 1.1365050 .7754281 .042473

P - $2.145644-0.884631-0.217877$

P $0.0550831 .135395-0.880768$

C -1.3891320.190342 -1.320669

C -1.807190 $0.175205-2.777598$

$\mathrm{H}-1.8560341 .196634-3.197064$

H -2.805209-0.262147 -2.939937

H - $1.119511-0.391206-3.435582$

C $-3.935119-1.041388-0.676196$

$\mathrm{H}-3.894262-1.483661-1.688637$

C -4.765123 -1.990138 0.190395

$\mathrm{H}-4.841481-1.5801571 .211494$

H -4.279982 -2.973834 0.282659

C -6.169770 -2.155571-0.384974

H -6.098408 -2.657457-1.367054

H -6.764076 -2.822051 0.261052

C -6.871073 $-0.812650-0.555042$

$\mathrm{H}-7.047950-0.3698150 .442063$

H -7.864199 -0.953778 -1.011430

C $-6.0314630 .152037-1.385238$

$\mathrm{H}-6.5276601 .133573-1.453833$

H -5.952404 -0.228202 -2.420133

C $-4.6305840 .316115-0.805927$

$\mathrm{H}-4.0234390 .996539-1.419124$

H -4.7015870.7846890.189027

C - $1.919410-0.3787081 .530634$

H -0.802913 -0.240904 1.540155

C -2.297622 -1.397084 2.607385

$\mathrm{H}-1.926800-2.4046862 .371346$

$\mathrm{H}-3.395678-1.4799512 .678852$

C -1.744009 -0.9501803 .958578$

$\mathrm{H}-2.034338-1.6697064 .741721$

H - $0.641863-0.9679463 .907464$

C -2.208056 0.4547374 .328514

$\mathrm{H}-3.2972360 .4336424 .517947$

$\mathrm{H}-1.7386270 .7746235 .273694$

C -1.914562 1.455842 3.217090 
$\mathrm{H}-0.8214951 .5701613 .083154$

$\mathrm{H}-2.3107182 .4503643 .479357$

C -2.489346 0.9926041 .884112

$\mathrm{H}-3.5907930 .9419351 .951266$

$\mathrm{H}-2.2439911 .7103971 .091587$

C - $1.542133-2.653983-0.246816$

$\mathrm{H}-2.209906-3.2601680 .391968$

C - $1.610522-3.194315-1.678963$

$\mathrm{H}-2.653692-3.216207-2.033362$

$\mathrm{H}-1.071426-2.497219-2.342359$

C -0.996620 -4.584521-1.807540

$\mathrm{H}-1.033204-4.911055-2.859573$

$\mathrm{H}-1.605351-5.308237-1.235279$

C $0.433503-4.612303-1.284880$

H $0.855823-5.626733-1.370162$

H $1.064492-3.954368-1.909212$

C $0.482973-4.1306060 .158300$

H $1.520804-4.1282610 .529398$

H $-0.072012-4.8432600 .796139$

C -0.117923 -2.737543 0.309655

H $0.512625-1.995703-0.205733$

H - $0.103565-2.4425611 .369442$

C $1.2654030 .775779-2.274065$

H $0.7379560 .995075-3.221885$

C $2.5167491 .651633-2.211937$

H $2.9853641 .511277-1.219577$

H $2.2495282 .716742-2.287587$

C $3.5156541 .301654-3.311989$

H $3.0740491 .544641-4.296167$

H $4.4166211 .930245-3.213665$

C $3.892309-0.174000-3.280950$

H $4.419975-0.386197-2.335119$

H $4.592344-0.413883-4.098617$

C $2.650677-1.053521-3.358669$

H $2.171690-0.925140-4.346979$

H $2.927570-2.119466-3.284513$

C $1.654846-0.699104-2.260784$

H $0.750639-1.320843-2.343940$

H $2.100845-0.923747-1.274305$

C $-0.3396272 .940466-1.286300$

$\mathrm{H}-0.0552763 .087040-2.346354$

C $0.5004573 .890225-0.427723$

H 0.2338713 .7170910 .630936

H $1.5691963 .638894-0.498915$

C $0.2652975 .351029-0.799843$

H $0.6272385 .525723-1.829937$

H $0.8613366 .010667-0.147131$

C -1.212008 5.718510-0.719122

$\mathrm{H}-1.3721276 .760012-1.043989$

H - 1.5373155 .6681790 .336248

C -2.064974 4.761922 -1.545031

$\mathrm{H}-3.1345215 .007211-1.433421$

H -1.826634 $4.893543-2.616508$

C -1.814996 3.308587-1.152696

$\mathrm{H}-2.4416962 .629954-1.748473$

$\mathrm{H}-2.1270313 .166430-0.104000$ 
C $3.544936-0.2785191 .760748$

C $3.265708-1.6149641 .417113$

C 4.6975650 .3025421 .218212

C $4.082134-2.3108530 .540321$

H $2.373212-2.1035891 .806344$

C $5.505564-0.4108790 .331505$

H 4.9693331 .3290181 .457522

C $5.220521-1.725457-0.033249$

H $3.819521-3.3406920 .281385$

H $6.3877780 .082637-0.085832$

N 2.663224 0.4417362 .633117

C $2.203626-0.3161353 .793789$

H $1.718607-1.2486293 .488341$

H $3.042449-0.5564194 .476229$

H 1.4596870 .2827324 .334773

C 3.1349871 .7587503 .044494

H 4.0674551 .6941533 .641120

H 3.3035662 .3934962 .166530

H 2.3541262 .2339253 .651088

C $6.072513-2.485009-1.006674$

H $6.410260-3.445055-0.585121$

H $5.514068-2.714805-1.929273$

H $6.964047-1.910106-1.294265$

$\mathrm{ArCl}=1-$ Chloro-3,5-dimethoxybenzene

L1-Pd(ArCl)

$E=-2984.24850733$

Pd $1.2057430 .186887-0.758756$

P - 2.5396940 .0329040 .026287

P 0.1999020 .3765811 .302681

C - 1.5718750 .3607261 .411417

C -2.216683 0.8959652 .674124

$\mathrm{H}-1.7975960 .4199543 .579659$

$\mathrm{H}-3.3024710 .7185192 .717807$

$\mathrm{H}-2.0855881 .9865972 .813390$

C $-4.191869-0.5754690 .597674$

$\mathrm{H}-4.5955100 .2926211 .150782$

C -5.190866 -0.899091-0.515000

$\mathrm{H}-4.830007-1.770337-1.087596$

H -5.274094 -0.066585-1.230205

C $-6.563726-1.2191910 .071641$

$\mathrm{H}-6.966773-0.3108830 .555308$

H -7.266470 -1.476965 -0.737194

C -6.492705 -2.346121 1.095976

$\mathrm{H}-6.207566-3.2823880 .582564$

$\mathrm{H}-7.486361-2.5256571 .537325$

C $-5.468391-2.0457902 .184943$

$\mathrm{H}-5.392580-2.8926112 .885948$

H -5.808956 -1.179333 2.780881

C $-4.098240-1.7374581 .590910$

H -3.364338 -1.510946 2.376882

H -3.721631 -2.632179 1.070950

C - $1.739803-1.154907-1.127872$ 
H $-0.722962-0.688046-1.210540$

C -2.307174 -1.265451-2.544147

$\mathrm{H}-2.488760-0.278145-2.990873$

H -3.281368 -1.783622 -2.519137

C - $1.335994-2.050161-3.424951$

$\mathrm{H}-1.753492-2.153593-4.439727$

$\mathrm{H}-0.409051-1.460970-3.529564$

C -1.011417 -3.422749 -2.844582

$\mathrm{H}-1.917937-4.054756-2.884506$

H - $0.255725-3.929058-3.467507$

C -0.534364 -3.329207-1.399548

H $0.443804-2.818153-1.357893$

$\mathrm{H}-0.382410-4.336706-0.979117$

C -1.522391 -2.547912 -0.542901

$\mathrm{H}-2.484807-3.088909-0.510468$

$\mathrm{H}-1.164959-2.4672880 .491245$

C - $2.9556731 .494986-1.054769$

H $-3.6586281 .155318-1.836824$

C -3.637964 2.581216-0.217578

$\mathrm{H}-4.5986112 .2163300 .180656$

H -3.001743 2.802264 0.656821

C -3.867754 3.864125-1.010170

$\mathrm{H}-4.3246184 .626502-0.358286$

$\mathrm{H}-4.5968603 .667387-1.817282$

C -2.573536 4.383845 -1.622584

$\mathrm{H}-2.7639725 .300533-2.203936$

$\mathrm{H}-1.8745634 .664564-0.814102$

C -1.927969 3.317697-2.497065

$\mathrm{H}-0.9726113 .676483-2.912676$

$\mathrm{H}-2.5854833 .112565-3.362029$

C - $1.6858592 .025619-1.725967$

H -0.920669 2.194148 -0.952083

$\mathrm{H}-1.2511981 .273932-2.399967$

C 0.7195341 .9963202 .093313

H 0.2498562 .0304003 .094819

C 2.2354482 .0984542 .270089

H 2.7160611 .9510051 .286475

H 2.6035811 .2923862 .923376

C 2.6572173 .4469712 .847994

H 2.2727303 .5390833 .880466

H 3.7567333 .4941272 .921402

C 2.1301344 .6083252 .014583

H 2.5993754 .5763541 .014442

H 2.4187585 .5719772 .465641

C 0.6171614 .5188581 .857531

H 0.1391764 .6510332 .845529

H 0.2445365 .3383671 .220255

C 0.2038183 .1738501 .272309

H -0.890758 3.111492 1.176529

H 0.6115473 .0788310 .248761

C $0.889341-0.8176002 .596165$

H $1.061291-0.2185053 .510859$

C $2.233230-1.3837702 .128851$

H $2.061911-1.9474901 .193353$

H $2.920686-0.5728331 .846480$

C $2.867878-2.2886703 .179286$ 
H $3.131520-1.6859794 .068024$ H $3.816139-2.7067822 .799116$ C $1.922470-3.4099213 .595527$ H $2.372953-4.0225914 .393856$ H $1.766264-4.0852712 .734307$ C $0.572539-2.8571944 .040975$ H $-0.115029-3.6823734 .290879$ H $0.706191-2.2725924 .969602$ C $-0.045852-1.9633352 .969638$ $\mathrm{H}-1.024314-1.5796603 .293610$ H $-0.240702-2.5741472 .072710$ Cl $1.4561910 .728945-3.862636$ C $2.3929680 .091365-2.514714$ C $3.1187950 .992862-1.693667$ C $2.647940-1.301784-2.461981$ C $4.1563470 .474516-0.884089$ H $3.0393462 .072269-1.821660$ C $3.654173-1.772949-1.620221$ H $2.103878-1.999699-3.096700$ C $4.443331-0.889603-0.856279$ H $5.237642-1.271705-0.221379$ O $3.813182-3.114807-1.589043$ O $4.8277351 .395395-0.163246$ C $4.732222-3.669285-0.692240$ H $4.497617-3.3994430 .352999$ H $4.659986-4.758871-0.801553$ H $5.768656-3.360564-0.917202$ C 5.7808640 .9640970 .766902 H 6.1507961 .8621641 .277434 H 5.3372720 .2832781 .514600 H 6.6311370 .4562540 .278507

TS (oxidative addition)

$E=-2984.23060934$

Pd $0.8287820 .269808-1.425435$

P -2.686374 0.0357590 .153334

P 0.2914000 .3688290 .810499

C - 1.4130110 .2326571 .298063

C - 1.7866090 .5179972 .742730

$\mathrm{H}-1.2971801 .4300353 .127922$

$\mathrm{H}-1.514670-0.2969783 .438968$

$\mathrm{H}-2.8646640 .6907472 .883732$

C -4.149833 -0.707940 1.008880

$\mathrm{H}-4.4500720 .0743871 .729158$

C -5.352419 -0.964317 0.097421

H -5.092723 -1.744982 -0.637714

H -5.611652 -0.063952 -0.480597

C $-6.559256-1.4243970 .911492$

$\mathrm{H}-6.882232-0.5986831 .571270$

H -7.407198 -1.633313 0.239306

C $-6.235173-2.6490551 .759340$

$\mathrm{H}-6.030069-3.5059261 .092112$

H -7.107349 -2.934010 2.369615

C -5.017718 -2.406064 2.644363 
H -4.768280 -3.315853 3.213812

H -5.256846 -1.627883 3.392052

C -3.811494 -1.959650 1.824565

$\mathrm{H}-2.944174-1.7719272 .471900$

H -3.517522 -2.773395 1.144212

C -2.206574 -0.967693-1.309327

$\mathrm{H}-1.190797-0.537893-1.512924$

C -3.028026 $-0.818158-2.592018$

$\mathrm{H}-3.2253790 .235056-2.832856$

H -4.013049 -1.301345 -2.469769

C -2.279320 -1.470227 -3.754015

$\mathrm{H}-2.870830-1.378584-4.679416$

$\mathrm{H}-1.340462-0.913205-3.921872$

C -1.950219 -2.932916 -3.476571

$\mathrm{H}-2.889482-3.515302-3.440644$

H - $1.356536-3.352341-4.304799$

C - $1.211844-3.098331-2.153438$

H $-0.217939-2.619614-2.221790$

H - $1.037739-4.164913-1.937337$

C $-1.980816-2.448514-1.008786$

$\mathrm{H}-2.955848-2.955423-0.894749$

$\mathrm{H}-1.440794-2.567571-0.061986$

C -3.360738 $1.614034-0.578312$

$\mathrm{H}-4.2006741 .349226-1.245583$

C -3.8811672.534604 0.529320

$\mathrm{H}-4.7231102 .0636021 .061589$

H -3.077802 2.675949 1.273541

C -4.316942 $3.894571-0.007172$

$\mathrm{H}-4.6495134 .5337860 .826877$

H -5.194650 $3.760684-0.665475$

C $-3.2017894 .571969-0.792693$

H -3.544660 5.540835 -1.190418

$\mathrm{H}-2.3592914 .792625-0.112661$

C $-2.7133203 .671826-1.919601$

$\mathrm{H}-1.8788154 .145651-2.461339$

$\mathrm{H}-3.5266933 .537773-2.656295$

C -2.270163 2.310121-1.397179

$\mathrm{H}-1.3821022 .431256-0.759564$

H -1.939840 1.678386-2.234999

C 0.9549211 .9041821 .647195

H 0.7854651 .7843352 .732981

C 2.4553632 .0692161 .407951

H 2.6274722 .1348300 .321606

H 3.0120421 .1803921 .743498

C 3.0057653 .3182032 .088187

H 2.9254473 .2059493 .185337

H 4.0793293 .4214331 .860055

C 2.2499024 .5687621 .654407

H 2.4350104 .7439240 .579061

H 2.6307345 .4568592 .185129

C 0.7505634 .4112251 .880719

H 0.5500884 .3464612 .965899

H 0.2096375 .3014781 .518230

C 0.2136303 .1583961 .197065

H -0.865060 3.0458481 .385906

H 0.3318863 .2591770 .102716 
C $1.296235-0.9596151 .678569$

H $2.290726-0.8643181 .204407$

C $0.752448-2.3377071 .317576$

H -0.278198 -2.4176461.709000

H $0.684478-2.4220000 .221351$

C $1.610891-3.4673021 .875726$

H $2.584548-3.4578431 .358064$

H $1.147610-4.4407521 .643181$

C $1.824027-3.3260473 .377838$

H $2.495085-4.1179903 .750244$

H 0.859174 -3.466719 3.898976

C $2.375456-1.9488813 .727083$

H $2.500085-1.8466964 .818014$

H $3.383539-1.8375513 .285762$

C $1.478282-0.8353123 .191800$

H 1.9052970 .1431433 .459712

H $0.495743-0.8936193 .689314$

Cl $1.841489-0.099351-3.584274$

C $2.839676-0.290315-1.975186$

C $3.6930000 .775316-1.656957$

C $3.118322-1.601060-1.560092$

C $4.7280620 .547589-0.753445$

H $3.5168381 .781292-2.035016$

C $4.181826-1.802726-0.681680$

H $2.501961-2.444852-1.866533$

C $4.986345-0.736795-0.250907$

H $5.805318-0.9061010 .442313$

O $4.371716-3.078798-0.272265$

O $5.4362251 .640220-0.389659$

C $5.417983-3.3598000 .612214$

H $5.296164-2.8321091 .575240$

H $5.390752-4.4411680 .797708$

H 6.400709 -3.096279 0.182891

C 6.4466911 .5055850 .567109

H 6.8642322 .5079000 .727292

H 6.0533281 .1269421 .527468

H 7.2552210 .8357720 .224075

L1-Pd(Ar)-Cl

$E=-2984.28320382$

Pd $0.579679-0.1438541 .290013$

P $-2.583386-0.267396-0.216672$

P $0.3063070 .514012-0.886708$

C - $1.4053030 .364988-1.312453$

C -1.830370 $0.551051-2.763114$

H -0.954895 $0.742427-3.398059$

$\mathrm{H}-2.5050491 .411699-2.925338$

H -2.322563 -0.332135 -3.208725

C -4.228807 $0.253493-0.882162$

H -4.133601 -0.042349-1.942406

C $-5.472590-0.455474-0.341153$

H $-5.628824-0.1855490 .716123$

H -5.353453 -1.548487 -0.371812

C $-6.702436-0.055404-1.154525$ 
H -6.587308 -0.438641 -2.184691

H -7.598204 -0.545847 -0.740899

C -6.895375 $1.456161-1.199598$

H -7.137055 $1.820120-0.184510$

$\mathrm{H}-7.7596371 .712004-1.833261$

C -5.639422 2.165329-1.694092

H -5.777597 $3.258045-1.666006$

H $-5.4558971 .901436-2.751478$

C -4.424983 1.772014-0.860920

$\mathrm{H}-3.5140112 .282837-1.207759$

H -4.589943 2.0991280 .178501

C -2.354937 0.2565181 .538594

$\mathrm{H}-1.386772-0.2179011 .840791$

C -3.365318 -0.3407092 .526462$

H -3.469728 -1.426796 2.389326

H -4.3566640 .1037252 .330499$

C -2.939689-0.040365 3.962518

H -3.686528 -0.4536704 .659381$

H - $1.985722-0.5577444 .161104$

C -2.753903 1.4537114 .200770

$\mathrm{H}-3.7315751 .9653914 .121491$

H -2.392030 1.6277965 .226394

C -1.7855632.0570373.189869

$\mathrm{H}-0.7792131 .6325923 .350845$

$\mathrm{H}-1.7026423 .1464703 .332754$

C -2.2285971.7619941.761384

$\mathrm{H}-3.2080612 .2334941 .582427$

$\mathrm{H}-1.5321402 .1925061 .034259$

C -2.716860 -2.121046 -0.101256

$\mathrm{H}-3.641867-2.3371870 .462223$

C -2.855472 -2.712663 -1.507729

$\mathrm{H}-3.760040-2.323892-2.003560$

H - $1.999235-2.375679-2.116033$

C -2.893020 $-4.237225-1.488517$

$\mathrm{H}-2.953780-4.619351-2.520391$

H -3.814089 -4.572256 -0.977653

C - $1.682245-4.816143-0.768304$

$\mathrm{H}-1.737517-5.916384-0.747803$

H $-0.765946-4.560352-1.330070$

C - $1.579722-4.2609310 .645706$

H -0.685225 -4.650309 1.156718

$\mathrm{H}-2.448946-4.6073411 .234262$

C $-1.539821-2.7363780 .660643$

$\mathrm{H}-0.592719-2.3909200 .213453$

H - $1.515532-2.3884771 .703782$

C $1.266621-0.464942-2.163973$

H $0.772111-0.236795-3.126507$

C $2.744167-0.084686-2.307773$

H $3.248867-0.174983-1.336117$

H $2.8428490 .966433-2.616429$

C $3.460234-0.973476-3.321255$

H $3.052114-0.786591-4.332124$

H $4.526228-0.695252-3.359823$

C $3.304194-2.451239-2.986394$

H $3.780216-2.656647-2.010813$

H $3.825842-3.073718-3.731732$ 
C $1.829117-2.824771-2.916762$

H $1.373529-2.694723-3.915858$

H $1.707339-3.889059-2.655135$

C $1.088344-1.960162-1.904210$

H $0.015944-2.201068-1.910647$

H $1.456632-2.191503-0.891748$

C $0.9410872 .236512-1.182060$

H $2.0284952 .111471-1.034279$

C $0.4609513 .236317-0.134827$

H - $0.6260833 .387168-0.258268$

H 0.6111332 .8287530 .878728

C $1.1744104 .575742-0.288824$

H $2.2511784 .433892-0.084818$

H 0.8061185 .2903960 .465177

C $0.9934965 .141907-1.692829$

H $1.5543206 .084039-1.805224$

H -0.071917 5.394786-1.844415

C $1.4208514 .137851-2.757493$

H $1.2303834 .541242-3.765660$

H $2.5115203 .974260-2.687878$

C $0.7080462 .798341-2.584908$

H $1.0475432 .092248-3.359410$

H -0.375265 2.939561 -2.739811

Cl $0.676525-0.9417403 .488242$

C $2.523929-0.1231431 .079619$

C $3.189115-1.3398330 .952447$

C 3.2235881 .0800341 .072212

C $4.570467-1.3425650 .741720$

H $2.670728-2.2968331 .003953$

C 4.6083231 .0644840 .875622

H 2.7353632 .0447481 .206870

C $5.293989-0.1431160 .690842$

H $6.367637-0.1504260 .520776$

O 5.2131582 .2744090 .853077

O $5.137616-2.5590410 .566837$

C 6.5965842 .3361490 .667447

H $6.9020801 .917953-0.308882$

H 6.8729223 .3980760 .697132

H 7.1442931 .8046701 .466140

C $6.516215-2.6399340 .353082$

H $6.757943-3.7056200 .249978$

H $6.822034-2.116274-0.570778$

H $7.089960-2.2264641 .201550$

$$
\text { L1-Pd(Ar)-Cl(HNMe } 2)
$$

$E=-2658.60935171$

Pd $0.701609-0.1636831 .218447$

P -2.665510 -0.332363 -0.253056

P $0.2082480 .448489-0.973223$

C - $1.5056340 .207271-1.408877$

C $-1.8640110 .074536-2.880991$

$\mathrm{H}-1.461539-0.838801-3.359583$

$\mathrm{H}-1.4936700 .926217-3.473300$

H -2.949954 $0.043704-3.053845$ 
C -4.360487 $0.050996-0.892698$

H -4.429840 -0.592894 -1.788105

C $-5.508440-0.3569090 .033922$

H -5.492326 0.2750260 .938217

H -5.394753 -1.396724 0.376366

C -6.851451-0.189001-0.673208

H -6.904233 -0.905134 -1.513286

H -7.671624-0.455713 0.012697

C -7.040101 1.226964-1.205747

H -7.113652 $1.926299-0.353152$

H -7.993265 $1.305618-1.752965$

C -5.874982 $1.649622-2.094160$

$\mathrm{H}-6.0035162 .692232-2.426538$

H -5.866333 1.030839 -3.010090

C -4.541489 1.495432 -1.370253

H -3.701075 $1.797329-2.010844$

H -4.525553 2.173966 -0.504496

C -2.428759 0.4219891 .414392

H - 1.3202610 .3851671 .539069

C -3.032133 -0.313778 2.614013

H - $2.779612-1.3819462 .601316$

H -4.132981-0.246235 2.571956

C - 2.5118880 .3018903 .911824

H -2.970754-0.210786 4.772650

H - 1.4272560 .1009983 .957939

C - 2.7747501 .8011763 .987777

H -3.862998 1.9819944 .067267

H -2.323933 2.218865 4.902374

C -2.2437272.525739 2.756192

H - 1.1413142 .4646772 .734460

H -2.499524 3.5969772 .794467

C - 2.7943681 .9045011 .478385

H -3.8938972.004381 1.476270

$\mathrm{H}-2.4215122 .4283310 .588514$

C - $2.732250-2.1694310 .067046$

H -3.548834 -2.355247 0.787761

C -3.036805 -2.927092 -1.229228

H -4.020805 -2.635870 -1.629848

H -2.290976 -2.639291-1.990339

C -2.998323 -4.439333-1.030219

H -3.189154 -4.944982 -1.990683

H -3.820526 -4.735662 - 0.353731

C -1.672561-4.896089-0.435570

H - $1.677363-5.986545-0.277297$

H - $0.859485-4.687458-1.154208$

C - $1.386515-4.1653740 .869225$

H -0.407093 -4.464193 1.276059

H - $2.139243-4.4564131 .624580$

C -1.415577-2.653037 0.679685

H $-0.587306-2.3547420 .017717$

H -1.218594 -2.155056 1.641524

C $1.162339-0.449013-2.310264$

H $0.649718-0.217066-3.260959$

C $2.620727-0.004269-2.460401$

H $3.136793-0.082191-1.493340$

H $2.6669481 .054335-2.756821$ 
C $3.367746-0.845609-3.491592$

H $2.945401-0.661366-4.497138$

H $4.420875-0.521899-3.531795$

C $3.277407-2.333909-3.179634$

H $3.769829-2.533738-2.211038$

H $3.819751-2.921661-3.938537$

C $1.820273-2.772202-3.106989$

H $1.352717-2.644533-4.100808$

H $1.746463-3.845267-2.863654$

C $1.049719-1.956148-2.076127$

$\mathrm{H}-0.012588-2.247115-2.076361$

H $1.436909-2.184169-1.068569$

C $0.7236912 .213408-1.258957$

H $1.8252162 .155602-1.192994$

C $0.2600363 .128665-0.130178$

H -0.840960 $3.186661-0.150918$

H 0.5330552 .6897170 .844419

C $0.8360584 .532126-0.280652$

H $1.9351814 .483606-0.176169$

H 0.4739725 .1816420 .533373

C $0.4815345 .131028-1.637516$

H $0.9388076 .127429-1.752646$

H -0.612308 $5.283376-1.688355$

C $0.9083824 .214517-2.778804$

H $0.5986464 .637959-3.748649$

H $2.0116344 .154976-2.803166$

C $0.3413442 .806361-2.612789$

H $0.6870982 .162736-3.437803$

H - $0.7605802 .844016-2.679398$

C $2.649297-0.0726300 .960205$

C $3.363673-1.2565990 .766042$

C 3.3155811 .1535070 .931276

C $4.728814-1.2067030 .473880$

H $2.887328-2.2375210 .802628$

C 4.6853791 .1949860 .654385

H 2.8011802 .1006811 .098958

C 5.4059510 .0183230 .407270

H 6.4673920 .0548790 .175356

N $0.815598-0.6563533 .146261$

C $1.271605-1.9756513 .480005$

H $0.834591-2.7286732 .804625$

H $2.378122-2.0884273 .434020$

H $0.970854-2.2490424 .513954$

C 1.3633390 .3179204 .050187

H 2.4751150 .3529724 .041478

H 1.0008081 .3294223 .806414

H 1.0607030 .1022455 .097541

O 5.2462472 .4273830 .622560

O $5.328159-2.3996710 .238283$

C 6.6098152 .5435750 .345377

H $6.8638362 .153595-0.657144$

H 6.8498493 .6144410 .376751

H 7.2307952 .0195201 .094280

C $6.683745-2.420812-0.097614$

H $6.956234-3.473354-0.249560$

H $6.886515-1.863976-1.030443$ 
H $7.316096-2.0040780 .706883$

TS (reductive elimination)

$E=-2658.58957784$

Pd 0.7190850 .1233601 .184885

P -2.690999 -0.473197 -0.223050

P $0.0617550 .637029-0.964192$

C - $1.6264510 .242166-1.373994$

C -2.035750 $0.194213-2.837153$

$\mathrm{H}-1.9125651 .165428-3.344120$

H -3.090928 -0.085534 -2.976127

$\mathrm{H}-1.457912-0.541024-3.428462$

C $-4.438501-0.151327-0.743242$

H $-4.537104-0.731531-1.678641$

C $-5.501437-0.6790190 .223000$

H -5.443325 -0.1181961 .171396$

H $-5.323323-1.7363530 .472336$

C -6.898172 $-0.512756-0.370827$

H -6.986579 -1.157368 -1.264122

H -7.655079 -0.8708440 .345598$

C -7.176376 $0.933783-0.763395$

$\mathrm{H}-7.2078041 .5556180 .149740$

$\mathrm{H}-8.1709761 .019233-1.229974$

C -6.098392 $1.475084-1.696045$

$\mathrm{H}-6.2875962 .535446-1.928294$

H -6.140749 $0.934493-2.659244$

C $-4.7066151 .315595-1.093182$

$\mathrm{H}-3.9322491 .693749-1.775011$

$\mathrm{H}-4.6363431 .928077-0.180839$

C -2.413008 0.1303401 .494111

$\mathrm{H}-1.2976960 .0587991 .550269$

C -2.940172 -0.7150322 .656382$

$\mathrm{H}-2.727122-1.7829922 .516181$

$\mathrm{H}-4.037534-0.6189532 .721868$

C -2.301076 -0.234211 3.958934

$\mathrm{H}-2.675869-0.8347594 .803385$

$\mathrm{H}-1.211698-0.4082983 .896854$

C -2.557790 1.2484954 .204596

$\mathrm{H}-3.6369851 .4062124 .386801$

$\mathrm{H}-2.0375431 .5778265 .118484$

C -2.123568 2.0941093.012972

$\mathrm{H}-1.0251192 .0389472 .903578$

$\mathrm{H}-2.3743303 .1544913 .176937$

C -2.7613891.5994971.719545

$\mathrm{H}-3.8585801 .7057541 .790488$

$\mathrm{H}-2.4317832 .2042300 .866144$

C -2.610546 -2.331487 -0.064028

$\mathrm{H}-3.398126-2.6481650 .643685$

C -2.877246 -2.989585-1.421485

$\mathrm{H}-3.892347-2.749611-1.776517$

$\mathrm{H}-2.178202-2.565247-2.162543$

C $-2.700075-4.504008-1.370578$

H $-2.868411-4.931303-2.372428$

$\mathrm{H}-3.475065-4.938098-0.712844$ 
C -1.323804 -4.891238 -0.845086

$\mathrm{H}-1.225216-5.987636-0.795721$

H -0.550955 -4.538579 -1.551518

C - $1.074467-4.2682380 .521698$

$\mathrm{H}-0.061968-4.5139640 .881292$

H - $1.779362-4.7030051 .254124$

C - $1.245319-2.7538910 .487185$

$\mathrm{H}-0.460594-2.309382-0.142643$

$\mathrm{H}-1.078291-2.3381151 .491706$

C $1.104022-0.150718-2.302026$

H $0.6625000 .148012-3.270915$

C $2.5594240 .322571-2.273254$

H $2.9775830 .139652-1.271867$

H $2.6178121 .408324-2.441908$

C $3.413882-0.392314-3.316552$

H $3.082095-0.096703-4.329451$

H $4.460646-0.059021-3.221070$

C $3.324560-1.907776-3.185783$

H $3.751978-2.216980-2.216281$

H $3.925156-2.398641-3.969653$

C $1.874272-2.368891-3.256959$

H $1.466085-2.145580-4.260071$

H $1.808017-3.462507-3.129713$

C $1.025883-1.672177-2.199949$

H -0.023896 -1.994921 -2.275479

H $1.379423-1.971015-1.197826$

C $0.4101192 .447520-1.270952$

H $1.4936802 .511142-1.056338$

C - $0.2953263 .336517-0.252738$

$\mathrm{H}-1.3841973 .271530-0.426444$

H -0.108124 2.9548320 .764935

C $0.1487714 .790598-0.372999$

H $1.2184644 .861805-0.104997$

H -0.3958935 .4162390 .353582$

C - $0.0531775 .320906-1.788191$

H $0.3202556 .354827-1.871185$

$\mathrm{H}-1.1363235 .363402-2.005514$

C $0.6233494 .423839-2.818785$

H $0.4262614 .793291-3.838844$

H $1.7186804 .467927-2.677937$

C $0.1642192 .973714-2.684279$

H $0.6693992 .347243-3.436079$

H $-0.9164332 .918016-2.901245$

C $2.712115-0.1167691 .304371$

C $3.259836-1.3464310 .908855$

C 3.5048441 .0389891 .248465

C $4.562381-1.4018330 .414957$

H $2.684879-2.2720810 .933417$

C 4.8068760 .9666010 .752638

H 3.1228902 .0184401 .536938

C $5.356681-0.2506210 .323505$

H $6.369909-0.300218-0.065434$

N $1.557544-0.2789112 .981259$

C $1.758134-1.6043693 .483818$

H $1.350133-2.3550682 .792218$

H $2.829567-1.8474863 .654555$ 
H $1.229211-1.7323524 .449853$

C 2.0199310 .7427023 .872433

H 3.1076980 .6691234 .089770

H 1.8276811 .7420553 .457978

H 1.4802460 .6824994 .839027

O 5.4811902 .1408700 .695378

O $4.986559-2.6262890 .010192$

C $6.271469-2.752796-0.523189$

H $6.404875-3.811913-0.779653$

H $6.399211-2.147854-1.439137$

H $7.050901-2.4630590 .204170$

C 6.7767732 .1512260 .175603

H $6.8046471 .807343-0.874405$

H 7.125821 3.1915030 .214399

H 7.4671401 .5246570 .768952

L1-Pd-( $\left.\mathrm{ArNMe}_{2}\right)$

$E=-2658.64271696$

Pd 0.7714390 .8765581 .093744

P - $2.416160-0.878581-0.247359$

P $-0.2438841 .182725-0.870800$

C -1.660806 $0.210173-1.339346$

C -2.041525 $0.179621-2.806093$

$\mathrm{H}-2.0813471 .197587-3.234569$

H -3.034828 $-0.259730-2.990161$

$\mathrm{H}-1.336912-0.390720-3.442793$

C $-4.183129-1.094071-0.764381$

$\mathrm{H}-4.092178-1.525457-1.778229$

C $-5.008701-2.0799030 .064102$

H -5.133731 -1.685064 1.086293

H -4.493187 -3.047326 0.162555

C -6.385955 -2.287498 -0.561837

H -6.262608 -2.777878-1.544595

$\mathrm{H}-6.979370-2.9797480 .057378$

C -7.127290 -0.968256-0.746990

$\mathrm{H}-7.354865-0.5403250 .246335$

H -8.098062 -1.139935 -1.239637

C -6.293010 0.032281-1.539327

$\mathrm{H}-6.8207450 .996430-1.618653$

H -6.163098 $-0.336086-2.573393$

C -4.921028 $0.239485-0.907401$

$\mathrm{H}-4.3154480 .947463-1.490494$

$\mathrm{H}-5.0459560 .6939910 .088704$

C -2.265223 -0.3435191 .501088$

$\mathrm{H}-1.153147-0.1799621 .547310$

C -2.659622 -1.356523 2.576757

$\mathrm{H}-2.258037-2.3581872 .367416$

$\mathrm{H}-3.757713-1.4625162 .609164$

C $-2.165824-0.8813233 .941366$

H - $2.471367-1.5963894 .722840$

$\mathrm{H}-1.062593-0.8788413 .931445$

C -2.669336 0.5190624 .275709

$\mathrm{H}-3.7638410 .4792094 .427972$

H -2.239171 0.8602545 .232122 
C -2.357307 1.5115553 .161459

$\mathrm{H}-1.2627801 .6465533 .063132$

H -2.781985 2.5011373 .396536

C -2.876904 1.0188621 .816354

$\mathrm{H}-3.9783920 .9439761 .848952$

$\mathrm{H}-2.6221741 .7317551 .022423$

C -1.757872 -2.627743 -0.230395

$\mathrm{H}-2.428833-3.2439180 .395351$

C - $1.765223-3.191673-1.654995$

$\mathrm{H}-2.795739-3.248730-2.041846$

$\mathrm{H}-1.225715-2.489665-2.312817$

C -1.107849 -4.565363 -1.741430

$\mathrm{H}-1.100096-4.907636-2.789274$

H -1.715760 -5.297449 -1.178979

C $0.303101-4.545071-1.168144$

H $0.755784-5.548862-1.224087$

H $0.937853-3.879102-1.780506$

C $0.294401-4.0413070 .268489$

H $1.321514-3.9963430 .661177$

H - $0.262332-4.7597600 .898728$

C $-0.351820-2.6643820 .375817$

H $0.278148-1.913275-0.126493$

H -0.381987 -2.353344 1.430737

C $1.0049970 .826716-2.231502$

H $0.4985861 .049486-3.190100$

C $2.2572341 .698394-2.141279$

H $2.7135081 .547915-1.145114$

H $1.9947742 .765049-2.208907$

C $3.2680271 .360686-3.235076$

H $2.8439281 .632119-4.219470$

H $4.1750451 .975153-3.107172$

C $3.629710-0.119385-3.237024$

H $4.152235-0.357546-2.295150$

H $4.328536-0.346974-4.059939$

C $2.383586-0.990948-3.328598$

H $1.901546-0.843200-4.312552$

H $2.654766-2.059967-3.275575$

C $1.393560-0.647814-2.222163$

H $0.490147-1.269809-2.304612$

H $1.843142-0.877670-1.238374$

C $-0.6536772 .976023-1.311806$

$\mathrm{H}-0.3531513 .110181-2.369150$

C $0.1575543 .951615-0.454249$

$\mathrm{H}-0.1265773 .7925420 .602018$

H $1.2309663 .714952-0.500855$

C -0.091266 $5.402432-0.855717$

H $0.2864155 .564735-1.882196$

H $0.4840106 .081418-0.204202$

C $-1.5747115 .750470-0.807005$

$\mathrm{H}-1.7434756 .783755-1.153154$

$\mathrm{H}-1.9175055 .7142550 .243390$

C - $2.4002844 .767485-1.629847$

H -3.474896 4.999277 -1.539913

$\mathrm{H}-2.1461884 .883924-2.699480$

C -2.136029 3.325061-1.207848

$\mathrm{H}-2.7438372 .626196-1.800042$ 
$\mathrm{H}-2.4610083 .197437-0.161141$

C $3.163791-0.0048861 .898773$

C $3.038550-1.3725621 .605365$

C 4.2135030 .7197681 .320063

C $3.903378-1.9729290 .695774$

H $2.233289-1.9803822 .009562$

C 5.0747150 .1004360 .412702

H 4.3705381 .7778321 .509811

C $4.940073-1.2527820 .088493$

H $5.612812-1.729771-0.618731$

N 2.227676 0.6269682 .775436

C $1.756268-0.1971233 .882976$

H $1.290970-1.1184423 .518693$

H $2.581381-0.4608424 .572791$

H 0.9904380 .3626434 .434588

C 2.5959561 .9593773 .240614

H 3.5214581 .9408423 .849709

H 2.7304852 .6373952 .390306

H 1.7719312 .3541033 .847624

O $6.0196820 .901229-0.126002$

O $3.661575-3.2785020 .433925$

C $4.376641-3.911289-0.588086$

H $5.454084-3.983662-0.356318$

H $3.966654-4.925698-0.676209$

H $4.253274-3.391957-1.554885$

C $6.9505090 .351983-1.014545$

H $7.6238541 .167346-1.308073$

H $7.545998-0.449414-0.542026$

H $6.466252-0.050560-1.921356$

$\mathrm{ArCl}=p$-chlorobenzonitrile

L1-Pd(ArCl)

$E=-2847.53169880$

Pd -1.400394 0.3289490 .820076

P $2.312112-0.048912-0.073496$

P $-0.4744770 .117190-1.304453$

C $1.2908660 .017948-1.456415$

C $1.8936210 .245760-2.828694$

H $1.418315-0.395921-3.592912$

H $2.9696840 .018892-2.874315$

H $1.7910511 .286220-3.192793$

C $3.922202-0.815216-0.567243$

H $4.318238-0.091795-1.303556$

C $4.962401-0.9293950 .549122$

H $4.611234-1.6544861 .302848$

H 5.0925780 .0297251 .073058

C $6.302242-1.400161-0.011925$

H $6.700576-0.621267-0.687158$

H $7.034193-1.5051660 .805096$

C $6.166213-2.710748-0.778603$

H $5.887546-3.514307-0.072861$

H $7.136201-3.002861-1.212034$

C $5.101027-2.613136-1.865217$ 
H 4.979166 -3.583222 -2.373161

H 5.430199 -1.896668 -2.639863

C $3.763652-2.154142-1.293995$

H $3.000255-2.076316-2.080612$

H $3.395992-2.913167-0.586148$

C $1.536948-0.9502071 .332448$

H $0.531181-0.4551371 .355882$

C $2.157215-0.7723192 .719596$

H 2.3674090 .2824792 .943016

H $3.123744-1.3025182 .766151$

C $1.214437-1.3376673 .780349$

H $1.670845-1.2344574 .778015$

H $0.299410-0.7205763 .794095$

C $0.853127-2.7950263 .512586$

H $1.753683-3.4204283 .653462$

H $0.117596-3.1470544 .254707$

C $0.324309-3.0000432 .097317$

H -0.650721 -2.494512 1.980762

H $0.148933-4.0705491 .903262$

C $1.284318-2.4309761 .060832$

H $2.240974-2.9798391 .110879$

H $0.890218-2.5653150 .045678$

C 2.8092721 .5877170 .669105

H 3.5300171 .3900221 .482909

C $3.4936822 .454349-0.392636$

H $4.4279151 .980274-0.734233$

H $2.8348802 .516620-1.276111$

C 3.7915063 .8618030 .114484

H $4.2470874 .458344-0.692434$

H 4.5436473 .8054390 .922336

C 2.5376274 .5433670 .646489

H 2.7777655 .5506301 .023253

H $1.8183774 .681524-0.180916$

C 1.8918613 .7023221 .739227

H 0.9646084 .1748372 .101378

H 2.5741023 .6514552 .607661

C 1.5815322 .2905601 .255345

H 0.7958822 .3290340 .485173

H 1.1498801 .7075642 .081564

C $-0.9660071 .573110-2.378423$

H - $0.5290221 .379791-3.376298$

C -2.478956 1.714271-2.545168

$\mathrm{H}-2.9342421 .799913-1.541565$

$\mathrm{H}-2.9063730 .810747-3.004596$

C -2.849189 2.932757 -3.387311

$\mathrm{H}-2.4878872 .780359-4.420616$

$\mathrm{H}-3.9459703 .021872-3.454380$

C -2.241480 4.214771-2.832077

$\mathrm{H}-2.6845224 .427551-1.841764$

$\mathrm{H}-2.4932285 .073043-3.476130$

C $-0.7314874 .078434-2.682218$

H $-0.2735913 .971866-3.682497$

$\mathrm{H}-0.3008704 .992161-2.239533$

C $-0.3702192 .866442-1.832047$

H $0.7218022 .765281-1.744324$

H -0.751907 $3.014352-0.804664$ 


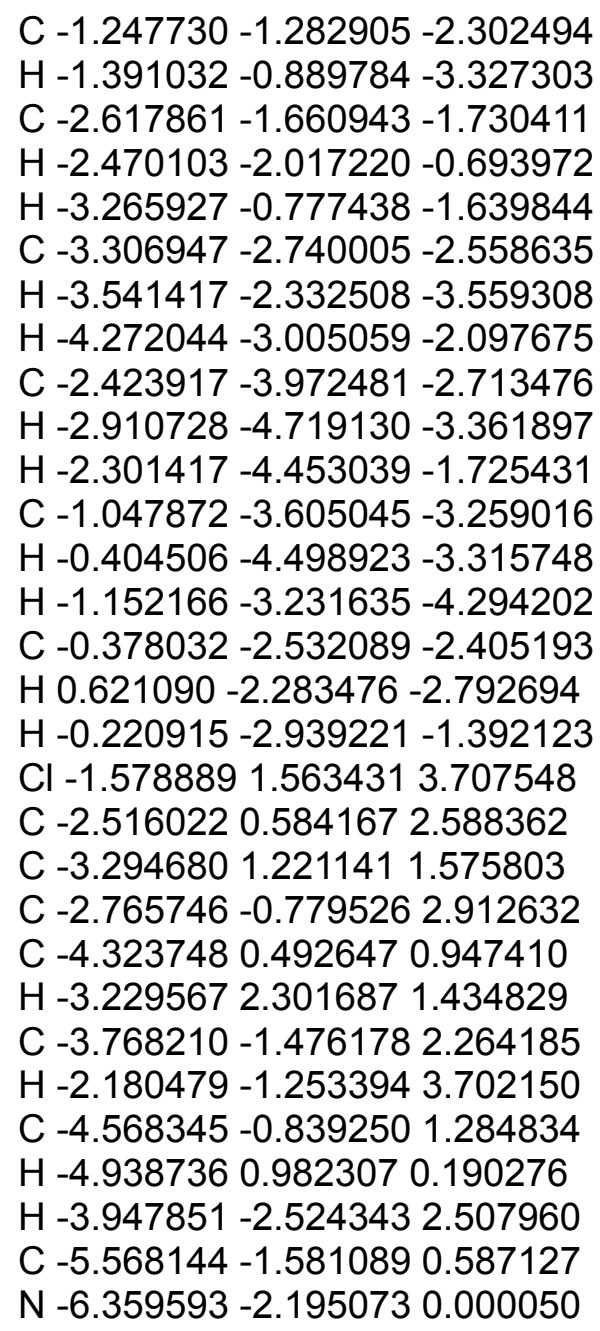

TS (oxidative addition)

$E=-2847.51464662$

Pd -1.166665 0.5219041 .358548

P $2.361831-0.113767-0.148047$

P $-0.5643340 .428352-0.871794$

C $1.1288390 .139214-1.325590$

C $1.5473200 .348191-2.771330$

H $1.1310551 .278998-3.195024$

H $1.229633-0.468069-3.445710$

H $2.6373590 .439310-2.895364$

C $3.783273-0.985928-0.950929$

H $4.150811-0.243651-1.682178$

C $4.948195-1.300714-0.009230$

H $4.624127-2.0478910 .735346$

H $5.254635-0.4074730 .556599$

C $6.138085-1.853513-0.790133$

H $6.526985-1.062126-1.456316$

H $6.957214-2.104705-0.097089$

C $5.752217-3.069488-1.624610$

H $5.480837-3.900265-0.948121$

H $6.615821-3.419353-2.212766$

C $4.569401-2.765843-2.537478$

H $4.273193-3.668078-3.096264$ 
H $4.870713-2.017903-3.293458$

C $3.379255-2.229984-1.748300$

H $2.536268-2.001881-2.414331$

H $3.025002-3.011377-1.059153$

C $1.774187-1.0414961 .327935$

H $0.780077-0.5486081 .483871$

C $2.565493-0.8956022 .629935$

H 2.7962020 .1546272 .852366

H $3.533886-1.4188272 .546297$

C $1.762835-1.4911553 .785741$

H $2.330995-1.3969574 .725280$

H $0.840624-0.8959393 .914341$

C $1.391492-2.9479773 .533393$

H $2.311550-3.5606923 .532830$

H $0.766384-3.3311974 .356295$

C $0.680843-3.1191882 .196122$

$\mathrm{H}-0.298961-2.6107632 .227722$

H $0.478224-4.1842051 .997838$

C $1.492756-2.5185061 .055147$

H $2.448298-3.0656320 .968990$

H $0.964739-2.6366100 .101065$

C 3.1418771 .4284260 .556941

H 3.9338761 .1156881 .261116

C $3.7765392 .265460-0.557797$

H $4.5907411 .707799-1.047408$

H $3.0133252 .454659-1.333227$

C $4.3141473 .597145-0.043072$

H $4.7298724 .178934-0.881735$

H 5.1542963 .4052640 .648922

C 3.2398024 .3943280 .684370

H 3.6553415 .3402691 .067565

H $2.4429374 .668642-0.030334$

C 2.6359293 .5749811 .817093

H 1.8292774 .1366772 .315445

H 3.4088743 .3918032 .586037

C 2.0906792 .2433451 .314281

H 1.2418332 .4239740 .639216

H 1.6718241 .6678822 .153088

C -1.095948 $1.977922-1.772315$

$\mathrm{H}-0.9360951 .800064-2.851291$

C -2.579700 2.274179-1.551573

$\mathrm{H}-2.7461712 .396250-0.466302$

$\mathrm{H}-3.2035561 .421271-1.861011$

C -3.019349 3.535724 -2.288584

H -2.9394013.365992 -3.377819

$\mathrm{H}-4.0838623 .739453-2.085996$

C -2.164580 4.737626-1.903564

$\mathrm{H}-2.3385114 .977583-0.838632$

H -2.468854 $5.628589-2.476774$

C - $0.6825144 .447015-2.110441$

H -0.482854 4.319490 -3.190018

H $-0.0714285 .304070-1.781336$

C -0.255669 3.184901-1.369031

H $0.8111892 .975252-1.541439$

H $-0.3719563 .342579-0.281162$

C -1.666623 $-0.850692-1.697005$ 
H -2.659587 -0.645795 -1.255840

C -1.259476 -2.254245 -1.260206

$\mathrm{H}-0.233389-2.446210-1.623326$

$\mathrm{H}-1.219893-2.295707-0.159725$

C -2.202103 -3.325837-1.797577

H -3.198753 -3.198804 -1.340706

$\mathrm{H}-1.845828-4.325463-1.497158$

C -2.346239 -3.241392 -3.311753

$\mathrm{H}-3.071298-3.989327-3.670876$

$\mathrm{H}-1.377947-3.488003-3.785367$

C -2.770255 -1.841443 -3.737907

$\mathrm{H}-2.845871-1.778320-4.835963$

$\mathrm{H}-3.781626-1.636563-3.343025$

C -1.800673 -0.781535-3.219410

$\mathrm{H}-2.1396900 .215501-3.539921$

H $-0.811723-0.938325-3.681952$

L1-Pd(Ar)-Cl

$E=-2847.56481953$

Pd $0.824948-0.2286711 .310680$

P - $2.316220-0.258821-0.241420$

P $0.5859900 .558875-0.826157$

C $-1.1172160 .429213-1.281213$

C - $1.5202260 .692087-2.726829$

$\mathrm{H}-0.6356060 .909298-3.340359$

$\mathrm{H}-2.1875671 .564058-2.853247$

$\mathrm{H}-2.011623-0.164079-3.222346$

C $-3.9475740 .313548-0.896220$

$\mathrm{H}-3.8441430 .067456-1.968460$

C $-5.203945-0.407371-0.402171$

$\mathrm{H}-5.369608-0.1843700 .664592$

H -5.095185 -1.499069-0.481657

C -6.420232 0.042905-1.209728

$\mathrm{H}-6.297241-0.293724-2.255115$

H -7.325336 -0.456724 -0.829015

C $-6.5974381 .556902-1.186571$

$\mathrm{H}-6.8482601 .876010-0.158733$

$\mathrm{H}-7.4511521 .850735-1.817845$

C $-5.3288532 .275516-1.632830$

H -5.456642 $3.367002-1.555034$

H -5.135072 $2.059660-2.699225$

C $-4.1283941 .831420-0.804848$

$\mathrm{H}-3.2084502 .348795-1.116436$

H -4.302964 2.110775 0.246666

C -2.1071240.156921 1.545596

$\mathrm{H}-1.135661-0.3251681 .828061$

C -3.117669 -0.512826 2.486299

H -3.206797 -1.589572 2.282406

H -4.112570 -0.069859 2.307058

C $-2.710075-0.2960953 .942561$

$\mathrm{H}-3.460587-0.7579364 .603843$

$\mathrm{H}-1.754358-0.8173264 .120786$

C - 2.5410901 .1818314 .274754

$\mathrm{H}-3.5228031 .6884344 .219053$ 
$\mathrm{H}-2.1892631 .2952535 .312171$

C -1.571666 1.8567583.311380

$\mathrm{H}-0.5614811 .4345373 .453809$

$\mathrm{H}-1.5019462 .9359453 .522247$

C -2.001767 1.6465191.864114

$\mathrm{H}-2.9872422 .1142011 .710042$

$\mathrm{H}-1.3085162 .1323311 .169826$

C -2.456822 -2.114196 -0.241510

$\mathrm{H}-3.386299-2.3606620 .301662$

C -2.587022 -2.621593-1.681431

$\mathrm{H}-3.485853-2.200116-2.160437$

$\mathrm{H}-1.724269-2.255394-2.263190$

C -2.632283 -4.144502-1.750995

$\mathrm{H}-2.688190-4.465452-2.803630$

H -3.558305 -4.503781 -1.266503

C -1.428962 -4.770593-1.058449

$\mathrm{H}-1.490019-5.869732-1.101462$

H $-0.508079-4.488311-1.599893$

C - $1.332211-4.2984370 .385853$

H $-0.442978-4.7214890 .879008$

$\mathrm{H}-2.206229-4.6738480 .948652$

C - $1.286289-2.7774160 .489622$

$\mathrm{H}-0.336238-2.4103450 .066214$

$\mathrm{H}-1.266184-2.4909361 .551419$

C $1.567961-0.340961-2.140581$

H $1.096342-0.038602-3.093953$

C $3.0527700 .024071-2.234380$

H $3.551124-0.182948-1.276278$

H $3.1786441 .099775-2.426198$

C $3.749501-0.767493-3.338438$

H $3.338625-0.467958-4.320307$

H $4.820096-0.507926-3.356335$

C $3.567093-2.268928-3.158019$

H $4.057633-2.581958-2.218851$

H $4.066689-2.819559-3.971236$

C $2.088264-2.628633-3.096500$

H $1.617467-2.401615-4.070585$

H $1.955807-3.710952-2.932544$

C $1.373716-1.849283-1.999073$

H 0.298972 -2.079339-2.006983

H $1.754862-2.168019-1.014122$

C $1.2174662 .298675-1.009409$

H $2.3030472 .178799-0.843307$

C 0.6917903 .2338030 .075094

H -0.394817 3.366003 -0.070077

H 0.8236782 .7774801 .070534

C 1.3747754 .5960390 .010605

H 2.4489304 .4721780 .239548

H 0.9668895 .2610720 .789007

C $1.2220075 .229866-1.367424$

H $1.7635256 .188413-1.413897$

H $0.1559885 .466905-1.538070$

C $1.7041684 .291363-2.467602$

H $1.5358204 .742104-3.459200$

H $2.7957024 .146957-2.373426$

C $1.0148732 .931298-2.387348$ 
H $1.3906582 .274164-3.187541$

$\mathrm{H}-0.0660463 .060633-2.565794$

Cl $0.902325-1.1735103 .449121$

C $2.770740-0.2015591 .166372$

C $3.454939-1.4172941 .048745$

C 3.4945250 .9918171 .247158

C $4.840562-1.4349430 .942704$

H $2.904319-2.3591871 .041979$

C 4.8823860 .9799201 .145759

H 2.9803261 .9443811 .385030

C $5.565121-0.2327090 .971946$

H $5.372278-2.3827720 .836836$

H 5.4457311 .9139791 .195720

C $6.988971-0.2444240 .831449$

N $8.142586-0.2525980 .708824$

L1-Pd(Ar)-Cl(HNMe $\left.{ }_{2}\right)$

$E=-2521.89315466$

Pd $0.981782-0.3108781 .181169$

P - $2.414185-0.282208-0.265388$

P $0.4730560 .517825-0.935695$

C -1.245813 0.340554-1.371337

C - $1.6155470 .354471-2.847221$

$\mathrm{H}-1.230018-0.514568-3.413740$

$\mathrm{H}-1.2379001 .253559-3.359307$

H -2.702917 $0.354929-3.012435$

C -4.101556 $0.201273-0.852333$

$\mathrm{H}-4.192121-0.350366-1.805507$

C $-5.253166-0.2687160 .040006$

$\mathrm{H}-5.2161560 .2708261 .001700$

H -5.162288 -1.339633 0.277605

C $-6.5961110 .000014-0.635261$

H -6.672208 $-0.628050-1.541358$

$\mathrm{H}-7.417753-0.3137790 .028452$

C $-6.7530881 .465911-1.023122$

$\mathrm{H}-6.8032742 .078861-0.104913$

H -7.707707 1.621383 -1.550781

C $-5.5844041 .946154-1.876783$

$\mathrm{H}-5.6891573 .019217-2.103515$

H -5.597947 $1.421000-2.849315$

C $-4.2494821 .689543-1.185163$

$\mathrm{H}-3.4073682 .031475-1.803136$

H $-4.2087602 .280228-0.257858$

C -2.152037 0.2926791 .467918

$\mathrm{H}-1.0446180 .2158961 .582545$

C -2.768853 -0.5500552 .588137$

$\mathrm{H}-2.538736-1.6162652 .465260$

H -3.867873 -0.456143 2.556351

C -2.236464 -0.082089 3.941425

$\mathrm{H}-2.706231-0.6712524 .745380$

$\mathrm{H}-1.156635-0.3083193 .965753$

C -2.467272 1.4064484 .172346

$\mathrm{H}-3.5512901 .6009374 .271930$

H -2.008364 1.7179555 .124370 
C -1.9207912.242338 3.020826

$\mathrm{H}-0.8198192 .1603322 .990442$

H -2.153252 3.3091013.169756

C -2.485255 1.7682511 .687316

$\mathrm{H}-3.5823271 .8907731 .700059$

$\mathrm{H}-2.1038022 .3747640 .855940$

C -2.516156-2.139411-0.130965

$\mathrm{H}-3.329848-2.3805740 .576317$

C -2.848251 -2.759489-1.492009

$\mathrm{H}-3.830008-2.411410-1.850288$

H -2.104718 -2.413399-2.230730

C -2.839266 -4.284366 -1.441842

$\mathrm{H}-3.049741-4.689829-2.444636$

$\mathrm{H}-3.660896-4.629263-0.788210$

C $-1.517599-4.823012-0.910355$

$\mathrm{H}-1.543515-5.923269-0.858810$

$\mathrm{H}-0.707591-4.561837-1.615254$

C -1.203310 -4.2295930 .456335$

H - $0.226565-4.5867050 .820545$

$\mathrm{H}-1.954132-4.5785231 .188500$

C - $1.203793-2.7054450 .417075$

$\mathrm{H}-0.376379-2.359044-0.222462$

H -0.989590 -2.308755 1.421646

C $1.402190-0.266193-2.355014$

H $0.8962780 .078555-3.274368$

C $2.8735940 .142734-2.467462$

H $3.398589-0.084161-1.527833$

H $2.9608801 .228788-2.619723$

C $3.569224-0.582361-3.616583$

H $3.130731-0.254383-4.577175$

H $4.632112-0.293236-3.644252$

C $3.430419-2.094546-3.497108$

H $3.945416-2.433213-2.579960$

H $3.930138-2.597052-4.341035$

C $1.962921-2.496590-3.426871$

H $1.469022-2.237851-4.381373$

H $1.861539-3.588491-3.310918$

C $1.249146-1.785511-2.283481$

H $0.179593-2.047465-2.280026$

H $1.658204-2.135091-1.318893$

C $1.0115692 .294412-1.061431$

H $2.1115412 .220688-0.981801$

C 0.5275753 .1154100 .129396

$\mathrm{H}-0.5718423 .1853540 .082187$

H 0.7675152 .5934751 .071506

C 1.1162204 .5215360 .114699

H 2.2123094 .4567480 .242173

H 0.7375255 .1017180 .972199

C $0.8017275 .237729-1.194303$

H $1.2713296 .234606-1.212627$

H $-0.2888525 .406794-1.257525$

C $1.2476734 .416595-2.399026$

H $0.9647344 .923925-3.335945$

H $2.3506184 .347577-2.404692$

C $0.6635403 .006020-2.367289$

H $1.0193352 .432304-3.238072$ 
H -0.436095 $3.062977-2.451843$

C $2.927775-0.1664270 .975839$

C $3.693050-1.3180600 .735131$

C 3.5920081 .0639581 .088434

C $5.069945-1.2413880 .560532$

H $3.207906-2.2947980 .669028$

C 4.9695701 .1520020 .918482

H 3.0287161 .9752741 .301083

C 5.7209670 .0000090 .639088

H $5.652295-2.1428700 .357437$

H 5.4745372 .1173470 .996690

N $1.078349-1.0058153 .045093$

C $1.523441-2.3559523 .243216$

H $1.106071-3.0282112 .476503$

H $2.631083-2.4671873 .222196$

H $1.192020-2.7401084 .230861$

C $1.610473-0.1371094 .059692$

H $2.722320-0.1111964 .080301$

H 1.2579360 .8968503 .917959

H $1.283601-0.4614715 .070361$

C 7.1345010 .0892260 .440054

N 8.2807170 .1620980 .274026

TS (reductive elimination)

$E=-2521.87705134$

Pd $0.953340-0.0559681 .196839$

P -2.416709 $-0.440868-0.294884$

P $0.3456300 .732972-0.884652$

C $-1.3399780 .397096-1.347846$

C $-1.7314380 .514967-2.812272$

$\mathrm{H}-1.5585221 .526645-3.213563$

$\mathrm{H}-2.7964380 .301095-2.987257$

$\mathrm{H}-1.179326-0.180879-3.471933$

C -4.158122 -0.065521-0.794669

H -4.239328 -0.534767 -1.791874

C -5.229038 -0.7094930 .089073$

$\mathrm{H}-5.188221-0.2634071 .097453$

H $-5.045576-1.7875650 .215174$

C $-6.619117-0.485422-0.501442$

H -6.690762 -1.025960 -1.462618

H -7.382030 -0.9271840.159588

C -6.9041530.994120 -0.732626

$\mathrm{H}-6.9533991 .5093700 .243872$

$\mathrm{H}-7.8930141 .124290-1.200738$

C -5.818775 $1.644432-1.583399$

$\mathrm{H}-6.0143192 .722554-1.698626$

H -5.842542 $1.214618-2.601419$

C -4.434498 $1.429748-0.980355$

$\mathrm{H}-3.6537061 .888733-1.602784$

H -4.385535 $1.936279-0.004346$

C -2.160228 -0.021935 1.483537

$\mathrm{H}-1.044348-0.0585261 .546830$

C -2.666147 -1.009336 2.538310

H -2.409809 -2.045910 2.283874 
H -3.767113 -0.9614612.593880

C -2.061774 -0.655323 3.896619

H -2.427368 -1.358426 4.662015

$\mathrm{H}-0.966502-0.7886043 .833616$

C -2.372190 0.7811874 .301421

$\mathrm{H}-3.4580570 .8827174 .482561$

$\mathrm{H}-1.8753541 .0236625 .254514$

C -1.952962 1.7681343.218234

$\mathrm{H}-0.8520431 .7635503 .121921$

H -2.242073 2.7947473 .494520

C -2.5589261.4008781.868249

$\mathrm{H}-3.6593681 .4611141 .936330$

$\mathrm{H}-2.2433762 .1092191 .092701$

C -2.337287 -2.304613 -0.337407

$\mathrm{H}-3.115639-2.6926010 .344225$

C -2.626435 -2.815861-1.752569

$\mathrm{H}-3.643938-2.534537-2.067260$

$\mathrm{H}-1.933451-2.322758-2.456074$

C -2.462887 $-4.328897-1.859605$

$\mathrm{H}-2.647557-4.649475-2.897580$

$\mathrm{H}-3.234346-4.821286-1.240073$

C $-1.084615-4.780060-1.394819$

H -0.996934 -5.876528 -1.457808

H - $0.317184-4.365003-2.072823$

C - $0.811282-4.3010780 .024704$

H $0.202860-4.5917840 .344060$

$\mathrm{H}-1.510962-4.8008130 .719464$

C -0.967082 -2.789262 0.144670

H -0.190324 -2.291633-0.454455

H -0.778487 -2.475789 1.182595

C $1.3934130 .118672-2.303144$

H $0.9481290 .536645-3.224824$

C $2.8488680 .587731-2.227217$

H $3.2844360 .272503-1.266611$

H $2.9043271 .686372-2.248065$

C $3.6867410 .021870-3.370824$

H $3.3327910 .445947-4.328759$

H $4.7340560 .343675-3.254040$

C $3.602965-1.497519-3.437285$

H $4.052473-1.922125-2.522225$

H $4.194489-1.880514-4.284514$

C $2.154123-1.956019-3.542925$

H $1.729989-1.610035-4.503412$

H $2.093460-3.057214-3.555682$

C $1.315953-1.403675-2.396007$

H 0.265656 -1.716099 -2.502881

H $1.676278-1.829564-1.443086$

C $0.7000892 .565184-0.943913$

H $1.7841482 .600612-0.725127$

C -0.0075703 .3097450 .182914$

$\mathrm{H}-1.0954783 .264493-0.000243$

H 0.1780022 .7977021 .142409

C 0.4304114 .7687060 .255570

H 1.5002794 .8098880 .529451

H -0.115909 5.290070 1.058954

C $0.2237405 .479704-1.077285$ 
H $0.5926426 .516944-1.023595$

H -0.859907 $5.545785-1.285557$

C $0.9026764 .729523-2.217688$

H $0.7017045 .228506-3.179929$

H $1.9981014 .760746-2.074726$

C $0.4510943 .272057-2.275663$

H $0.9593232 .752851-3.103233$

H -0.629634 $3.239152-2.497292$

C $2.929909-0.2934391 .295988$

C $3.516470-1.4723180 .789761$

C 3.7445090 .8519021 .417638

C $4.840465-1.4930830 .379557$

H $2.917553-2.3787570 .684958$

C 5.0676930 .8334431 .003808

H 3.3239431 .7816591 .805379

C $5.635094-0.3365300 .468246$

H 5.270332 -2.409422 -0.031201

H 5.6747191 .7384691 .077469

N $1.754968-0.6737552 .951551$

C $1.967624-2.0492763 .287370$

H $1.550857-2.7131972 .516382$

H $3.041794-2.3074223 .414960$

H $1.454248-2.2965244 .238070$

C 2.2073410 .2374743 .960313

H 3.2982280 .1596274 .160783

H 1.9902461 .2769903 .676662

H 1.6803960 .0464914 .916541

C $6.988112-0.3485360 .015355$

N $8.087640-0.357852-0.358804$

L1-Pd-( $\left.\mathrm{ArNMe}_{2}\right)$

$E=-2521.92479207$

Pd 1.0317240 .8711431 .092572

P -2.137625 -0.935114 -0.232557

P $0.0250881 .128081-0.900923$

C - $1.3796470 .130033-1.345165$

C -1.748848 $0.058342-2.814238$

$\mathrm{H}-1.7736311 .063311-3.272722$

$\mathrm{H}-2.746287-0.373136-2.992812$

$\mathrm{H}-1.046217-0.539186-3.426966$

C $-3.895041-1.181329-0.766834$

H -3.780637 -1.623584 -1.773486

C $-4.722890-2.1698770 .056283$

H -4.877998 -1.7652351.070418

$\mathrm{H}-4.195596-3.1282740 .177665$

C -6.082061 -2.405934 -0.598847

H -5.928268 -2.906423 -1.572088

$\mathrm{H}-6.678849-3.0998030 .015110$

C -6.839099-1.100827 -0.817392

H -7.097180 -0.664962 0.164954

H -7.794930 -1.293911-1.330629

C $-6.002496-0.096236-1.602071$

H $-6.5429510 .858530-1.704622$

H -5.842505 -0.473747 -2.628522 
C -4.649199 $0.139496-0.940566$

$\mathrm{H}-4.0409750 .851332-1.516388$

$\mathrm{H}-4.8058760 .6012870 .047776$

C -2.013237-0.352183 1.504573

H -0.903226 -0.1947821 .571435$

C -2.439839-1.336885 2.594575

$\mathrm{H}-2.027827-2.3421692 .425455$

$\mathrm{H}-3.537620-1.4470512 .590131$

C - $1.999430-0.8252623 .964395$

$\mathrm{H}-2.336005-1.5201184 .751122$

H $-0.896778-0.8231623 .999835$

C -2.513454 0.5832354 .242915

$\mathrm{H}-3.6130530 .5484374 .351787$

$\mathrm{H}-2.1217550 .9482155 .206893$

C -2.155883 1.5459973 .116530

$\mathrm{H}-1.0582631 .6781623 .059014$

H -2.5861942.5420743.309473

C -2.6277031.019941 1.766690

$\mathrm{H}-3.7294710 .9498761 .762687$

$\mathrm{H}-2.3442111 .7111250 .963616$

C - $1.463353-2.675647-0.162239$

$\mathrm{H}-2.138723-3.2827570 .467461$

C - $1.442680-3.272210-1.573244$

H -2.465219 -3.338881-1.978745

$\mathrm{H}-0.889871-2.585157-2.235713$

C $-0.784122-4.647117-1.613000$

$\mathrm{H}-0.754508-5.015045-2.651310$

$\mathrm{H}-1.403041-5.365714-1.045283$

C $0.616356-4.612271-1.015303$

H $1.068955-5.616682-1.034077$

H $1.263323-3.964792-1.633940$

C $0.576856-4.0691230 .406476$

H $1.593239-4.0229790 .829509$

H $0.006962-4.7687321 .045561$

C $-0.066880-2.6882270 .465993$

H $0.569257-1.951529-0.050865$

H -0.115895 -2.347666 1.511383

C $1.2781000 .760725-2.249653$

H $0.7504420 .913287-3.210107$

C $2.4880731 .693375-2.228046$

H $2.9773731 .604634-1.240298$

H $2.1740562 .743147-2.332156$

C $3.4811431 .351606-3.336766$

H $3.0079351 .548567-4.316369$

H $4.3557122 .021073-3.277663$

C $3.922935-0.104909-3.275926$

H $4.499628-0.268089-2.350418$

H $4.604057-0.341329-4.109140$

C $2.724940-1.046088-3.279204$

H $2.214339-0.985508-4.258017$

H $3.061462-2.090377$-3.167153

C $1.735020-0.692692-2.174773$

H $0.859372-1.357994-2.215169$

H $2.204994-0.855292-1.187021$

C $-0.4025922 .909260-1.368941$

H - $0.0884363 .029729-2.423617$ 
C $0.3875763 .906356-0.516864$

H 0.0891893 .7646540 .538166

H $1.4636103 .677575-0.545766$

C $0.1321035 .347441-0.948030$

H $0.5258775 .494431-1.970613$

H $0.6900186 .043418-0.299486$

C -1.355159 5.682043 -0.930733

$\mathrm{H}-1.5267896 .707127-1.298608$

$\mathrm{H}-1.7157945 .6621960 .114137$

C -2.157953 4.677059-1.749564

$\mathrm{H}-3.2357084 .901219-1.682795$

$\mathrm{H}-1.8860504 .775500-2.816490$

C -1.889662 3.245128 -1.295468

$\mathrm{H}-2.4802272 .529671-1.885263$

H -2.233051 $3.135406-0.252824$

C $3.291134-0.0562321 .928780$

C $3.177698-1.4561861 .768639$

C 4.3406770 .5967331 .245450

C $4.008883-2.1448220 .904827$

H $2.401421-2.0104022 .291759$

C $5.172467-0.0982830 .384449$

H 4.4916311 .6678251 .359373

C $5.014862-1.4784560 .186654$

H $3.880980-3.2210910 .776128$

H $5.9600740 .436169-0.149752$

N 2.4281430 .6504462 .808678

C $1.897549-0.1064533 .936799$

H $1.346025-0.9877203 .594492$

H $2.702352-0.4279124 .625335$

H 1.1890470 .5297334 .481068

C 2.8670741 .9812163 .217544

H 3.8077091 .9403393 .800647

H 3.0029082 .6271342 .342900

H 2.0790862 .4307913 .833547

C $5.850371-2.189788-0.725135$

N $6.519257-2.768665-1.477176$

$\mathrm{ArCl}=$ 1-Chloro-4-methoxybenzene

L1-Pd(ArCl)

$E=-2869.79651086$

$\mathrm{Pd}-1.3260680 .1964411 .018180$

P $2.327304-0.091899-0.105858$

P $-0.4658340 .591812-1.086315$

C $1.2727900 .377870-1.386022$

C $1.8802220 .905183-2.674004$

H $1.6369961 .968244-2.857890$

H $1.5455450 .351819-3.568706$

H $2.9796430 .856292-2.681183$

C $3.873864-0.794968-0.842936$

H $4.3237780 .076045-1.353081$

C $4.902109-1.2937430 .174843$

H $4.497192-2.1744580 .701845$

H $5.101680-0.5323390 .944447$ 
C $6.204109-1.681780-0.521958$

H $6.660809-0.774523-0.957528$

H 6.924915 -2.066678 0.217470

C $5.971517-2.707830-1.625293$

H $5.630607-3.655648-1.170417$

H $6.918007-2.935567-2.141631$

C $4.919921-2.228097-2.619665$

H $4.728679-3.001961-3.380415$

H $5.304222-1.346215-3.164130$

C $3.617953-1.855983-1.917898$

H $2.868155-1.499437-2.637277$

H $3.191287-2.756813-1.451000$

C $1.558445-1.2899861 .061960$

H $0.546314-0.8242831 .194197$

C $2.177723-1.4259192 .455011$

H $2.374275-0.4478112 .913260$

H $3.150606-1.9421572 .383752$

C $1.239732-2.2248733 .357698$

H $1.691470-2.3400804 .356269$

H $0.315166-1.6387343 .500648$

C $0.901408-3.5904012 .769313$

H $1.811244-4.2185782 .771255$

H $0.168965-4.1098713 .409203$

C $0.376660-3.4781901 .342409$

H -0.604981 -2.974053 1.344673

H $0.216720-4.4795720 .910599$

C $1.327531-2.6761720 .462707$

H $2.293007-3.2084580 .393497$

H $0.932287-2.588808-0.556912$

C 2.9534831 .2768240 .997011

H 3.6607100 .8333131 .721084

C 3.6959932 .3280380 .166446

H $4.5854941 .887396-0.311717$

H $3.0340922 .662053-0.651777$

C 4.1153853 .5332561 .002504

H 4.6110254 .2778260 .358440

H 4.8689873 .2139261 .745299

C 2.9301144 .1541471 .729846

H 3.2602925 .0066402 .345264

H 2.2191064 .5602300 .987781

C 2.2201163 .1152452 .587552

H 1.3388683 .5544673 .082193

H 2.8985772 .7857983 .395958

C 1.7872841 .9090581 .762422

H 1.0204242 .2162891 .037107

H 1.2883641 .1738392 .410870

C $-0.9032822 .284928-1.754681$

$\mathrm{H}-0.5635162 .326631-2.806012$

C -2.411447 2.540068 -1.729015

$\mathrm{H}-2.7666812 .403214-0.691260$

H -2.944299 $1.793691-2.337871$

C -2.762760 3.941296-2.222379

$\mathrm{H}-2.5063784 .021536-3.294645$

$\mathrm{H}-3.8514644 .102088-2.152823$

C -2.014535 5.020778-1.449850

H -2.354762 $5.015413-0.398179$ 
H -2.254713 6.019429-1.850025

C - $0.5115244 .773062-1.486322$

H $-0.1479424 .883369-2.524329$

H $0.0205735 .531967-0.888608$

C $-0.1722833 .376659-0.978750$

H $0.9135763 .201978-1.020319$

$\mathrm{H}-0.4608923 .2960870 .085168$

C - $1.474327-0.494906-2.234017$

H - $2.508728-0.203208-1.970329$

C - $1.333596-1.967058-1.863190$

$\mathrm{H}-0.301691-2.288069-2.091575$

$\mathrm{H}-1.477912-2.086644-0.776151$

C -2.319240 -2.838910-2.632896

H -3.345144 -2.587623 -2.308767

H -2.169811 -3.900263 -2.373833

C - $2.188352-2.636155-4.138532$

$\mathrm{H}-2.945774-3.229008-4.677506$

$\mathrm{H}-1.204452-3.017630-4.467942$

C -2.296349 -1.162644 -4.515940

$\mathrm{H}-2.150897-1.031693-5.601164$

$\mathrm{H}-3.318950-0.804680-4.294678$

C -1.295335 -0.309303 -3.739299

$\mathrm{H}-1.4020270 .749074-4.026079$

H -0.270850 -0.608601-4.019660

$\mathrm{Cl}-1.8364110 .4947074 .112563$

C -2.584032 -0.2399372 .693021$

C -3.3580750 .5539221 .799838$

C -2.721149-1.654645 2.651987

C $-4.274352-0.0780960 .918439$

$\mathrm{H}-3.4303941 .6324861 .955470$

C -3.588549 -2.246608 1.756977

H -2.150524 -2.263899 3.355250

C -4.373921-1.461104 0.882274

$\mathrm{H}-4.8806110 .5534550 .269442$

H -3.684106 -3.3324271 .704140$

O $-5.173133-2.1511220 .036419$

C -5.951233 -1.430789-0.874666

$\mathrm{H}-5.326826-0.819509-1.551612$

H -6.505158 -2.166978 -1.471052

H $-6.673157-0.767752-0.364753$

TS (oxidative addition)

$E=-2869.77874807$

$\mathrm{Pd}-1.0080240 .6475441 .422270$

P $2.407109-0.222805-0.163885$

P $-0.4672650 .570555-0.813861$

C $1.1919400 .179602-1.317874$

C $1.6040480 .426034-2.758492$

H $1.2360211 .396365-3.135541$

H $1.231388-0.340630-3.463162$

H $2.6960360 .460789-2.894975$

C $3.781574-1.111366-1.028657$

H $4.182835-0.340872-1.711418$

C $4.930641-1.539871-0.112524$ 
H $4.571876-2.3165980 .584384$ H $5.278130-0.6997010 .507869$

C $6.094342-2.095048-0.930089$ H $6.520701-1.280237-1.543074$ H $6.899918-2.429569-0.256606$ C $5.653886-3.233496-1.843127$ H $5.342724-4.093902-1.223156$ H $6.501367-3.583301-2.454440$ C $4.487977-2.814811-2.732055$ H $4.151970-3.662687-3.350404$ H $4.824870-2.031776-3.435718$ C $3.322354-2.280483-1.905946$ H $2.490766-1.971426-2.553285$ H 2.934215 -3.090152 -1.270329 C $1.762349-1.2221471 .241324$ H $0.782449-0.7087081 .416092$ C $2.537286-1.1860662 .560616$ H $2.788707-0.1592062 .856978$ H $3.494190-1.7265832 .453841$ C $1.702184-1.8365183 .662305$ H $2.256834-1.8174904 .614387$ H $0.792390-1.2283213 .815515$ C $1.303829-3.2646323 .308340$ H $2.210695-3.8963113 .278634$ H $0.658378-3.6877744 .095143$ C $0.608142-3.3302441 .954051$ H - $0.363223-2.8099932 .009614$ H $0.389683-4.3753261 .680465$ C $1.445562-2.6698380 .865805$ H $2.388400-3.2335120 .750866$ H $0.924572-2.709239-0.099115$ C 3.2744031 .2193430 .646422 H 4.0300430 .8122391 .342091 C $3.9830502 .077809-0.405481$ H $4.7697691 .498829-0.914889$ H $3.2483442 .360856-1.180204$ C 4.5953863 .3390240 .196371 H $5.0614203 .941922-0.599995$ H 5.4102093 .0516700 .885654 C 3.5632264 .1594770 .958264 H 4.0332135 .0509051 .404253 H 2.7978964 .5286190 .251840 C 2.8882153 .3141832 .030070 H 2.1108313 .8954052 .551807 H 3.6339513 .0328282 .796201 C 2.2660772 .0559491 .436545 H 1.4467942 .3373590 .759218 H 1.7896371 .4609172 .230277 C $-0.8970462 .193696-1.637685$ $\mathrm{H}-0.7815272 .050993-2.727540$ C -2.345759 2.595233-1.357246 H -2.466558 $2.691254-0.263218$ $\mathrm{H}-3.0441921 .803862-1.670868$ C -2.707067 $3.912744-2.036908$ $\mathrm{H}-2.6721113 .776966-3.133509$ H -3.745704 4.191462 -1.792965 
C -1.749960 $5.030636-1.639152$

$\mathrm{H}-1.8752265 .245296-0.562219$

$\mathrm{H}-1.9992255 .962174-2.173291$

C $-0.3013024 .633842-1.899770$

H -0.140699 4.530699-2.988536

H $0.3832515 .428711-1.559323$

C $0.0443383 .316384-1.214399$

H $1.0877403 .032269-1.420969$

H $-0.0354703 .443814-0.119536$

C -1.672995 -0.585067-1.673173

H $-2.637606-0.343477-1.189187$

C -1.344623 -2.034088 -1.328243

$\mathrm{H}-0.345623-2.270618-1.737813$

$\mathrm{H}-1.275579-2.140562-0.234351$

C -2.374822 -3.008414 -1.889828

H -3.344907 -2.840731-1.391409

$\mathrm{H}-2.079765-4.044870-1.655126$

C $-2.551625-2.831785-3.392867$

H -3.332122 -3.511258 -3.773135

H -1.614870 -3.112643 -3.908615

C $-2.889745-1.385527-3.732954$

H -2.991372 -1.256224 -4.823356

H -3.873329 -1.132285 -3.295532

C -1.841074 -0.420534-3.184729

$\mathrm{H}-2.1231760 .612139-3.440995$

H $-0.877155-0.613431-3.685109$

Cl -2.094470 0.7650053 .560169

C -2.940526 -0.070418 2.040934

C -3.949729 0.643109 1.381481

C $-2.989793-1.4759562 .069880$

C $-4.885052-0.0381960 .603670$

$\mathrm{H}-3.9707511 .7319361 .435662$

C -3.928922 -2.144626 1.302034

$\mathrm{H}-2.268707-2.0313092 .670677$

C $-4.865688-1.4361610 .537279$

$\mathrm{H}-5.6244330 .5414470 .049986$

$\mathrm{H}-3.951019-3.2357821 .272647$

O $-5.699979-2.180858-0.227091$

C -6.612032 -1.520551-1.054782

$\mathrm{H}-6.103857-0.859810-1.780756$

$\mathrm{H}-7.159905-2.296075-1.605297$

H -7.335127 $-0.918181-0.475985$

L1-Pd(Ar)-Cl

$E=-2869.83492459$

Pd -0.8543730 .6289651 .342816$

P $2.290880-0.263920-0.125366$

P $-0.4951600 .676403-0.927054$

C $1.1957680 .323577-1.323622$

C $1.7087690 .746598-2.688935$

H $1.3196121 .734491-2.987453$

H $1.4349930 .048066-3.501851$

H $2.8036560 .850361-2.723707$

C $3.845032-0.816962-0.954282$ 
H $4.2094760 .116984-1.419674$ C $4.933953-1.2903670 .013245$ H $4.613283-2.2310610 .492532$ H $5.089160-0.5636740 .824989$ C $6.247337-1.521846-0.730014$ H $6.617883-0.552013-1.108788$ H $7.012492-1.892758-0.029373$ C $6.078777-2.487338-1.897532$ H $5.832193-3.490237-1.504358$ H $7.029058-2.594897-2.444596$ C $4.966702-2.036303-2.837851$ H 4.824501 -2.771482 -3.645988 H 5.258280 -1.089229 -3.327494 C $3.654783-1.832503-2.086782$ H $2.858559-1.510532-2.770600$ H $3.332393-2.796554-1.667902$ C $1.585198-1.6069550 .932005$ H $0.540369-1.2464461 .061398$ C $2.173139-1.7782442 .336012$ H $2.217462-0.8236372 .876108$ H 3.208465 -2.155749 2.267058 C $1.315900-2.7541083 .139012$ H $1.746495-2.8828614 .144714$ H $0.321832-2.2966873 .287684$ C $1.181556-4.1030332 .443059$ H 2.168798 -4.600409 2.420960 H $0.514698-4.7652953 .017955$ C $0.673842-3.9497911 .014329$ H -0.370987 -3.595640 1.027313 H $0.660878-4.9246050 .500563$ C $1.524163-2.9620830 .223399$ H $2.546270-3.3703120 .139601$ H $1.137131-2.847489-0.798142$ C 2.8730541 .0312111 .083820 H 3.5462450 .5397851 .807481 C 3.6400892 .1401290 .359878 H $4.5667491 .741821-0.083052$ H $3.0220202 .505389-0.479165$ C 3.9826863 .3047981 .285035 H 4.4958854 .0929500 .710652 H 4.7024922 .9586882 .048760 C 2.7481303 .8625571 .982138 H 3.0290314 .6863152 .657509 H 2.0666534 .2959391 .228426 C 2.0233752 .7676562 .751384 H 1.1010683 .1401583 .222453 H 2.666957 2.408749 3.574100 C 1.6747521 .5895991 .850136 H 0.9264081 .9460221 .104566 H 1.2069290 .8104042 .474162 C $-0.8539012 .384136-1.583451$ H - $0.6831292 .335821-2.674028$ C -2.303269 $2.807050-1.347520$ $\mathrm{H}-2.4723712 .856647-0.258205$ H -3.008991 2.057253 -1.736515 C $-2.5853104 .173338-1.965497$ 
H -2.484683 4.102251 -3.063979

$\mathrm{H}-3.6290734 .467295-1.768393$

C -1.625989 5.233527-1.436305

$\mathrm{H}-1.8203735 .387998-0.359526$

$\mathrm{H}-1.8112296 .202539-1.927403$

C - $0.1731424 .809687-1.621583$

H $0.0649874 .775407-2.700321$

H $0.5056915 .556434-1.177676$

C $0.0888103 .437713-1.009713$

H $1.1359663 .135843-1.161054$

H -0.0669813 .4943980 .082638$

C - $1.635726-0.396261-1.936746$

$\mathrm{H}-2.619884-0.269417-1.452131$

C -1.227779 -1.858016-1.783533

H - $0.227297-1.987685-2.233329$

$\mathrm{H}-1.131211-2.109253-0.717769$

C -2.227405 -2.791789-2.453543

$\mathrm{H}-3.193831-2.716156-1.924154$

$\mathrm{H}-1.895926-3.838012-2.348214$

C -2.414087 -2.437024 -3.923920

$\mathrm{H}-3.179101-3.082860-4.384848$

H - $1.471569-2.635261-4.466691$

C -2.786375 -0.968782 -4.095529

H -2.872799 -0.714842 -5.164868

$\mathrm{H}-3.782916-0.793660-3.651316$

C - $1.779137-0.041317-3.417942$

H -2.101934 $1.002729-3.547896$

H $-0.800021-0.132691-3.919325$

$\mathrm{Cl}-1.0922570 .5728393 .670075$

C - $2.601209-0.2447451 .119280$

C -3.764302 0.4701100 .847962

C -2.697398 -1.629868 1.293716

C $-4.993748-0.1810500 .689873$

$\mathrm{H}-3.7397971 .5561520 .757306$

C -3.910636 -2.288366 1.133045

$\mathrm{H}-1.817306-2.2105251 .575094$

C -5.069892 -1.570327 0.814853

H -5.881779 0.4150690 .476105

H -3.987886 -3.370025 1.262756

O -6.203352 -2.294819 0.660899

C -7.390979-1.618954 0.372867

H -7.332120 -1.067123 -0.583285

$\mathrm{H}-8.178357-2.3798190 .292615$

H -7.666093 -0.906292 1.171388

$$
\text { L1-Pd(Ar)-Cl(HNMe } \left.{ }_{2}\right)
$$

$E=-2544.15732230$

Pd $0.904235-0.2758391 .193888$

P -2.468759 -0.331322 -0.284784

P $0.3932520 .579181-0.906810$

C - $1.3163610 .357105-1.367136$

C - $1.6758740 .391318-2.844831$

$\mathrm{H}-1.254267-0.449854-3.427866$

$\mathrm{H}-1.3270061 .314796-3.333546$ 
H -2.761349 $0.355735-3.019667$

C -4.168683 $0.107945-0.873079$

H $-4.235622-0.424320-1.839174$

C -5.309805 -0.4185240 .000791$

H -5.295026 0.0995540 .974798

H -5.186234 -1.491177 0.214599

C $-6.657172-0.177125-0.676027$

H $-6.708426-0.785934-1.596935$

H -7.472042 -0.532171-0.024733

C -6.858865 1.291651-1.030965

$\mathrm{H}-6.9331711 .881084-0.099070$

$\mathrm{H}-7.8152601 .429097-1.560603$

C -5.701365 1.828302 -1.866113

$\mathrm{H}-5.8391362 .902511-2.068839$

H -5.693138 $1.325509-2.850490$

C -4.362646 1.597893-1.172686

H -3.528395 $1.980371-1.777379$

$\mathrm{H}-4.3451722 .167827-0.231695$

C -2.240075 0.2114491 .463245

$\mathrm{H}-1.1317370 .1629941 .584689$

C $-2.839995-0.6718112 .561044$

$\mathrm{H}-2.585407-1.7293572 .413610$

H -3.940978 -0.602223 2.529596

C -2.319046 -0.222810 3.925247

$\mathrm{H}-2.773701-0.8419364 .715401$

$\mathrm{H}-1.233341-0.4220713 .943751$

C -2.588269 1.253745 4.190078

$\mathrm{H}-3.6771021 .4178444 .293669$

$\mathrm{H}-2.1371311 .5549475 .149191$

C -2.0634942.130310 3.058716

H -0.960838 2.077772 3.027903

$\mathrm{H}-2.3244453 .1868513 .232043$

C -2.612979 1.6722951 .713297

$\mathrm{H}-3.7130151 .7665871 .724612$

$\mathrm{H}-2.2435732 .3061330 .896918$

C -2.512374 -2.194158-0.192402

$\mathrm{H}-3.328218-2.4772230 .496769$

C -2.804731-2.791559-1.572506

H -3.791828 -2.466176 -1.937620

H -2.062020 -2.403425 -2.290818

C -2.747145 -4.316157-1.559567

H $-2.929286-4.703148-2.575246$

H -3.566840 $-4.703421-0.927469$

C $-1.416842-4.825232-1.020272$

$\mathrm{H}-1.407838-5.926831-0.996172$

H $-0.605209-4.520294-1.705443$

C - $-1.142872-4.2557240 .365057$

$\mathrm{H}-0.160936-4.5903600 .736551$

$\mathrm{H}-1.893611-4.6461131 .076247$

C -1.190774 -2.731942 0.362274

H -0.364416 -2.343994 -0.254276

$\mathrm{H}-1.003006-2.3533101 .378780$

C $1.358469-0.145221-2.335365$

H $0.8472560 .197251-3.252873$

C $2.8157160 .316460-2.427852$

H $3.3383770 .097716-1.485185$ 
H $2.8649971 .406609-2.567265$

C $3.548330-0.368603-3.578333$

H $3.109593-0.045711-4.540827$

H $4.600644-0.041095-3.589857$

C $3.462873-1.886123-3.477715$

H $3.975553-2.214795-2.555675$

H $3.991297-2.361515-4.320327$

C $2.009389-2.339732-3.432743$

H $1.519855-2.084996-4.390722$

H $1.944670-3.436161-3.332848$

C $1.257235-1.669802-2.289150$

H $0.197184-1.967670-2.304355$

H $1.665750-2.018603-1.324419$

C $0.8733062 .374870-0.999628$

H $1.9744852 .333619-0.914980$

C 0.3610183 .1584330 .204499

H - 0.7400253 .1970850 .155764

H 0.6155482 .6256371 .136435

C 0.9077004 .5814090 .217652

H 2.0047624 .5459550 .347507

H 0.5097175 .1345281 .084388

C $0.5758085 .312318-1.078915$

H $1.0148876 .323363-1.077117$

$\mathrm{H}-0.5192885 .449229-1.143223$

C $1.0500674 .527646-2.297143$

H $0.7552095 .044106-3.225574$

H $2.1546204 .491499-2.299173$

C $0.5085363 .099781-2.293284$

H $0.8843192 .553023-3.173041$

H - $0.5920833 .125580-2.381358$

C $2.855869-0.0778720 .985159$

C $3.650276-1.1932990 .716708$

C 3.4920301 .1662331 .103777

C $5.031366-1.0799060 .518891$

H $3.198012-2.1857590 .641230$

C 4.8643661 .2930560 .914301

H 2.9119872 .0620221 .339239

C 5.6456220 .1725720 .606473

H $5.610926-1.9774180 .297763$

H 5.3606422 .2626200 .998067

N $1.029492-1.0118473 .042528$

C $1.522538-2.3491453 .206919$

H $1.117952-3.0192862 .431172$

H $2.633102-2.4225183 .167260$

H $1.218522-2.7660604 .190811$

C $1.541292-0.1494064 .072262$

H $2.651502-0.0792034 .078106$

H 1.1475010 .8736003 .961210

H $1.241226-0.5108785 .079390$

O 6.9695180 .3937270 .419233

C $7.784877-0.6922550 .096287$

H $8.803890-0.301070-0.022793$

H $7.790592-1.4588790 .892603$

H $7.476817-1.174582-0.849716$ 
TS (reductive elimination)

$E=-2544.13567420$

Pd 0.9142760 .1043421 .323997

P $-2.374191-0.584038-0.408025$

P $0.2090551 .047743-0.668080$

C - $1.3934950 .544015-1.265439$

C -1.752058 $0.822263-2.716665$

$\mathrm{H}-2.7531640 .454144-2.986544$

$\mathrm{H}-1.0614090 .346434-3.438153$

$\mathrm{H}-1.7542561 .898567-2.954321$

C -4.132752 -0.384630 -0.953245

$\mathrm{H}-4.110676-0.708429-2.009435$

C -5.131021 -1.292167 -0.230748

H -5.191862 -0.996827 0.830545

H -4.797976 -2.341352 -0.245696

C $-6.516583-1.177741-0.861744$

$\mathrm{H}-6.474392-1.573800-1.892615$

$\mathrm{H}-7.231294-1.813662-0.314747$

C -7.004060 $0.266260-0.896169$

$\mathrm{H}-7.1618800 .6185180 .139450$

$\mathrm{H}-7.9840840 .327486-1.396205$

C -5.993982 $1.179143-1.582789$

H -6.339155 2.225161-1.554048

H -5.919658 $0.906563-2.651426$

C -4.614996 $1.069051-0.940604$

$\mathrm{H}-3.8872441 .717353-1.448207$

$\mathrm{H}-4.6717861 .4276550 .098699$

C -2.244513 -0.412667 1.419681

$\mathrm{H}-1.133360-0.3270001 .526983$

C -2.662394 -1.602021 2.288255

$\mathrm{H}-2.258129-2.5487891 .905863$

$\mathrm{H}-3.761164-1.7047162 .283612$

C -2.172367 -1.382358 3.719109

$\mathrm{H}-2.473974-2.2324174 .352221$

$\mathrm{H}-1.068371-1.3632703 .709766$

C -2.688402 -0.072607 4.303927

$\mathrm{H}-3.785927-0.1336914 .423822$

$\mathrm{H}-2.2743050 .0825625 .313291$

C -2.349626 1.1086583 .402396

$\mathrm{H}-1.2535451 .2486583 .376690$

$\mathrm{H}-2.7800062 .0401573 .804166$

C -2.842725 0.8762791 .978744

$\mathrm{H}-3.9442620 .7943611 .986430$

$\mathrm{H}-2.5863211 .7256821 .334198$

C -2.020334 -2.389868 -0.722347

H -2.758871 -2.985768 -0.156099

C -2.171185 -2.709174 -2.213033

$\mathrm{H}-3.206977-2.533871-2.544793$

$\mathrm{H}-1.536051-2.014491-2.789120$

C -1.771146 -4.145206 -2.537297

$\mathrm{H}-1.861021-4.319736-3.621744$

H -2.481425 -4.838233 -2.050645

C $-0.359765-4.457923-2.057700$

$\mathrm{H}-0.100415-5.505308-2.281105$

H $0.361573-3.830862-2.611948$ 
C -0.224037 -4.177798 -0.567207

H $0.808305-4.367770-0.230145$

H - $0.868026-4.878267-0.004296$

C - $0.613793-2.743149-0.230181$

H $0.103706-2.048195-0.691302$

H - $0.523371-2.5739980 .853038$

C $1.3691750 .798446-2.113433$

H $0.9122711 .314176-2.978212$

C $2.7529071 .404096-1.873962$

H $3.1849540 .961572-0.962594$

H $2.6776852 .486931-1.689795$

C $3.6902301 .157092-3.053087$

H $3.3168241 .704945-3.938351$

H $4.6862581 .573019-2.827237$

C $3.799537-0.324734-3.389221$

H $4.280031-0.848857-2.544905$

H $4.451337-0.474636-4.265651$

C $2.421963-0.926499-3.639301$

H $1.985327-0.476174-4.550024$

H $2.502839-2.008811-3.836400$

C $1.487142-0.683433-2.459717$

H $0.489067-1.097665-2.669132$

H $1.875648-1.216056-1.575566$

C $0.2710272 .911875-0.491642$

H $1.3197843 .083323-0.183508$

C -0.6152103 .3866610 .654884$

$\mathrm{H}-1.6678743 .1953340 .380787$

$\mathrm{H}-0.4053312 .7900051 .558735$

C - 0.4295154 .8736950 .937780

H 0.5987875 .0443331 .305012

$\mathrm{H}-1.1052815 .1936401 .748300$

C $-0.6631485 .713725-0.312876$

$\mathrm{H}-0.4743786 .779681-0.104516$

$\mathrm{H}-1.7268335 .637646-0.604166$

C $0.2027105 .233295-1.471975$

H -0.011604 5.819243 -2.381099

H $1.2666795 .411614-1.231246$

C -0.002999 $3.744747-1.743396$

H $0.6344263 .424087-2.582145$

H -1.047317 $3.580439-2.059317$

C $2.858894-0.4677371 .315855$

C $3.186407-1.6744760 .669425$

C 3.8674830 .4961701 .440060

C $4.440720-1.8701360 .106899$

H $2.438302-2.4636800 .570291$

C 5.1333850 .3030820 .877943

H 3.6617741 .4434721 .942244

C $5.425333-0.8784770 .191924$

H $4.680162-2.795128-0.422703$

H 5.8756801 .0968630 .972465

N $1.718182-0.7152422 .987234$

C $1.756951-2.1208383 .259458$

H $1.216624-2.6883342 .488860$

H $2.791162-2.5253953 .317461$

H $1.261519-2.3362334 .226970$

C 2.3542980 .0698814 .003407 
H $3.432984-0.1717534 .127577$ H 2.2692121 .1425623 .781204 H $1.859856-0.0993184 .980091$ O $6.606634-1.146602-0.421046$ C $7.588788-0.155653-0.422933$ H $8.442358-0.553134-0.987813$ H $7.2388950 .771830-0.913157$ H 7.9278820 .0969560 .598581

$$
\begin{aligned}
& \text { L1-Pd-(ArNMe }) \\
& E=-2544.18984861
\end{aligned}
$$

Pd 0.9311580 .9748461 .043625

P - $2.196473-0.978042-0.170593$

P $-0.0750981 .088081-0.945526$

C - $1.4611600 .039108-1.340984$

C $-1.833300-0.117328-2.802049$

$\mathrm{H}-1.9278400 .862597-3.304757$

H -2.799032 -0.624865 -2.954153

$\mathrm{H}-1.094819-0.692691-3.393908$

C -3.954024 -1.284800 -0.672809

$\mathrm{H}-3.848896-1.809633-1.640118$

C -4.756186 -2.205837 0.248299

H -4.893713 -1.715115 1.226529

H -4.215991 -3.145193 0.441771

C -6.126355 -2.509356 -0.353422

H -5.988706 -3.089929-1.283733

H -6.702775 -3.152947 0.330893

C $-6.900691-1.233773-0.665474$

$\mathrm{H}-7.140550-0.7171810 .281723$

H -7.865995 -1.477260 -1.138136

C $-6.090864-0.294410-1.552334$

H -6.642873 $0.643545-1.725017$

H -5.950024 -0.758588 -2.545605

C -4.725542 $0.009213-0.945045$

$\mathrm{H}-4.1388490 .671797-1.596526$

$\mathrm{H}-4.8633680 .5569680 .001058$

C -2.070249 -0.3037401 .529883$

H $-0.966702-0.0831701 .558528$

C - $2.412324-1.2440292 .686524$

$\mathrm{H}-1.958757-2.2372772 .559097$

H -3.503194 -1.405061 2.732729

C - $1.943204-0.6323084 .004969$

$\mathrm{H}-2.209430-1.2957124 .844266$

$\mathrm{H}-0.841638-0.5718013 .988082$

C -2.520137 0.7621014.225466

$\mathrm{H}-3.6113250 .6775094 .383044$

$\mathrm{H}-2.1100731 .2021365 .149745$

C -2.257869 1.6750023 .033246

$\mathrm{H}-1.1715471 .8567432 .921770$

H -2.733328 2.6572933 .187788

C -2.750466 1.0473091 .735171

$\mathrm{H}-3.8467170 .9180571 .778683$

$\mathrm{H}-2.5339841 .7043500 .883627$

C -1.488877 -2.702601-0.015405 
H -2.133931 -3.285348 0.666934

C - $1.491317-3.379663-1.389628$

$\mathrm{H}-2.523627-3.508368-1.752849$

H $-0.989298-2.712676-2.110659$

C $-0.782253-4.729710-1.377667$

H $-0.774779-5.154825-2.394489$

$\mathrm{H}-1.352096-5.437409-0.748231$

C $0.635482-4.609921-0.834232$

H $1.127191-5.596247-0.818709$

H $1.232434-3.969490-1.508355$

C $0.621778-3.9958810 .558961$

H $1.649304-3.8867340 .943899$

H $0.104873-4.6853031 .251826$

C -0.076403 -2.640400 0.573262

H $0.515159-1.9046650 .005934$

$\mathrm{H}-0.108633-2.2515811 .602116$

C $1.1892300 .663272-2.272457$

H $0.6825710 .804849-3.246195$

C $2.4209521 .567819-2.242324$

H $2.8787601 .493981-1.238147$

H 2.134216 2.621235 -2.381514

C $3.4388991 .178914-3.312371$

H $3.0024871 .366678-4.310862$

H $4.3280541 .826917-3.236940$

C $3.844165-0.286648-3.212485$

H $4.398784-0.445664-2.272372$

H $4.536167-0.550888-4.029485$

C $2.619384-1.193245-3.233109$

H $2.134392-1.132121-4.224867$

H $2.916639-2.248581-3.101677$

C $1.616103-0.796039-2.156762$

H $0.729564-1.446330-2.196356$

H $2.069098-0.940945-1.159040$

C - $0.5377302 .826839-1.527961$

$\mathrm{H}-0.2414282 .884115-2.593403$

C $0.2425993 .893390-0.754196$

H -0.038375 3.8130140 .311778

H $1.3226903 .686357-0.782375$

C - $0.0494735 .298013-1.273805$

H $0.3241015 .385937-2.310768$

H $0.5045456 .045582-0.681668$

C -1.542694 5.604427 -1.251588

$\mathrm{H}-1.7420456 .599501-1.682882$

$\mathrm{H}-1.8848505 .646273-0.201209$

C -2.338210 4.531678-1.987331

$\mathrm{H}-3.4193444 .737457-1.913809$

$\mathrm{H}-2.0882914 .565890-3.063690$

C -2.029678 3.138323-1.447286

$\mathrm{H}-2.6172162 .373968-1.975359$

H -2.347446 $3.089515-0.391703$

C 3.3722640 .2117921 .931024

C $3.206622-1.1453671 .618619$

C 4.4980640 .8655841 .407554

C $4.089716-1.8122880 .776982$

H $2.341503-1.6899201 .995656$

C 5.3788030 .2051340 .559043 
H 4.6846431 .9150741 .628430

C $5.182421-1.1353980 .223921$

H $3.899117-2.8597850 .542490$

H 6.2385370 .7263950 .134085

N 2.3937580 .8924792 .734600

C 1.9721300 .1567563 .922372

H $1.586302-0.8325793 .655375$

H 2.8082470 .0327214 .638657

H 1.1592000 .7092214 .411288

C 2.7269372 .2674293 .084149

H 3.6342912 .3246263 .719669

H 2.8759172 .8646202 .176525

H 1.8810392 .7019183 .631394

O $6.071756-1.686434-0.633442$

C $5.851530-2.996937-1.068522$

H $6.653406-3.233513-1.779492$

H $5.891175-3.721432-0.235184$

H $4.878611-3.099956-1.582017$

Amines

$\mathrm{R}=\mathrm{Et}$

L1-Pd(Ar)-Cl(HNR 2$)$

$E=-2508.27776073$

Pd $1.320125-0.1109551 .099270$

P - $2.287274-0.337723-0.230908$

P $0.5827420 .391275-1.076169$

C -1.149009 $0.131295-1.432794$

C -1.574201-0.045850 -2.882749

$\mathrm{H}-1.209092-0.982138-3.346620$

$\mathrm{H}-1.2232240 .777614-3.524328$

$\mathrm{H}-2.668054-0.065748-3.000793$

C -3.991729 $0.134159-0.784827$

H $-4.140815-0.520614-1.662117$

C $-5.103594-0.2100720 .209915$

$\mathrm{H}-5.0076800 .4251171 .107014$

H -5.018295 -1.251794 0.555141

C $-6.4755010 .013285-0.422667$

H -6.612058 -0.714336-1.243253

$\mathrm{H}-7.266847-0.1995190 .314056$

C -6.625295 $1.426572-0.974462$

$\mathrm{H}-6.6121692 .145558-0.135207$

H -7.604944 $1.542608-1.465289$

C -5.497067 $1.771783-1.940720$

H -5.597451 $2.810782-2.293441$

H -5.571523 $1.130733-2.838160$

C -4.131954 1.573025-1.290148

$\mathrm{H}-3.3168041 .813890-1.987560$

$\mathrm{H}-4.0345232 .275193-0.449381$

C - 1.9129150 .4091621 .415392

$\mathrm{H}-0.7972510 .4273191 .418312$

C -2.367712 -0.3601342.657963

H -2.052021-1.410805 2.618645 
H -3.471135 -0.3605512.706526

C -1.781992 0.286892 3.911009

$\mathrm{H}-2.126871-0.2595564 .803621$

$\mathrm{H}-0.6843470 .1658763 .869811$

C -2.148075 1.7623884 .021452

$\mathrm{H}-3.2354941 .8585354 .197834$

$\mathrm{H}-1.6530912 .2118614 .897762$

C - 1.7811032 .5297852 .756639

$\mathrm{H}-0.6852272 .5723072 .651300$

H -2.130953 3.5728402 .821522

C -2.3644811.8675071.516727

$\mathrm{H}-3.4659511 .8959611 .577738$

$\mathrm{H}-2.0792142 .4191380 .611406$

C -2.452244 -2.168031 0.081947

$\mathrm{H}-3.172453-2.3042250 .908687$

C - $2.976080-2.907314-1.153477$

$\mathrm{H}-3.983763-2.555691-1.424990$

$\mathrm{H}-2.320158-2.677808-2.011451$

C $-3.012983-4.417218-0.935174$

H -3.364382 -4.916701 -1.852586

H -3.756362 -4.648895 -0.150862

C -1.654732 -4.961410 -0.512762

$\mathrm{H}-1.714224-6.046995-0.333520$

$\mathrm{H}-0.933493-4.819767-1.338113$

C - $1.142929-4.2390790 .726372$

H -0.140343 -4.601643 1.002004

$\mathrm{H}-1.802794-4.4677061 .583229$

C - $1.098057-2.7322080 .507060$

H $-0.360596-2.496271-0.274486$

H $-0.730567-2.2298831 .412659$

C $1.456525-0.504631-2.468359$

H $0.869756-0.280844-3.376485$

C $2.892037-0.047678-2.743295$

H $3.502703-0.151177-1.835855$

H $2.9096671 .018931-3.012137$

C $3.530597-0.859759-3.866646$

H $2.999925-0.661045-4.816481$

H $4.570731-0.526758-4.014050$

C $3.485116-2.353810-3.574139$

H $4.082581-2.561169-2.668201$

H $3.947721-2.925077-4.395574$

C $2.050380-2.811257-3.346623$

H $1.476446-2.681018-4.282566$

H $2.015755-3.887477-3.108706$

C $1.380882-2.013355-2.233473$

H $0.326252-2.315977-2.138312$

H $1.862379-2.245737-1.267471$

C $1.0329982 .164456-1.433258$

H $2.1376352 .149057-1.416385$

C $0.5743983 .087201-0.309034$

$\mathrm{H}-0.5282053 .089867-0.287647$

H 0.9041122 .6865550 .663881

C $1.0739154 .512828-0.510434$

H $2.1774344 .521373-0.447283$

H 0.7111255 .1618940 .303609

C $0.6418665 .063007-1.865246$ 
H $1.0479706 .076014-2.019923$

H - $0.4589055 .164138-1.876951$

C $1.0673234 .138592-3.000496$

H $0.7029984 .524093-3.967089$

H $2.1704734 .127576-3.066686$

C $0.5698342 .711517-2.781536$

H $0.9071942 .065961-3.608235$

H $-0.5343942 .702229-2.800210$

C 3.2309540 .0001130 .634426

C $3.981323-1.1690000 .458917$

C 3.8768891 .2355280 .504366

C $5.333993-1.1041530 .120172$

H $3.510533-2.1484310 .574577$

C 5.2307401 .2986640 .167900

H 3.3254782 .1664500 .657803

C $5.9636580 .130310-0.036246$

H $5.898243-2.029921-0.024032$

H 5.7143992 .2740100 .063411

H $7.0213060 .181299-0.305319$

N $1.767120-0.4632603 .030162$

C $2.323187-1.7500493 .371022$

H $3.401014-1.8140523 .092784$

H $2.310459-1.8607294 .476015$

C 2.4883400 .5612453 .752714

H 3.5586830 .5923403 .446541

H 2.5056510 .2896694 .829440

C $1.586540-2.9234642 .761228$

H $0.536346-2.9402083 .091822$

H $1.590319-2.8628131 .660860$

H $2.053135-3.8770253 .053209$

C 1.8965761 .9489193 .619315

H 1.8323802 .2472592 .561364

H 0.8827531 .9888334 .043364

H 2.5160042 .6921224 .144606

TS (reductive elimination)

$E=-2508.25681380$

Pd 1.2939680 .0698171 .013923

P $-2.333317-0.400344-0.169001$

P $0.4591470 .413753-1.120859$

C $-1.2709430 .100529-1.426178$

C $-1.739077-0.094990-2.859039$

$\mathrm{H}-1.4830860 .761416-3.503100$

H -2.830856 -0.210768 -2.936225

$\mathrm{H}-1.311457-0.990954-3.348351$

C -4.079542 -0.014002 -0.652972

$\mathrm{H}-4.249336-0.691228-1.509359$

C -5.123766 -0.372484 0.407547

$\mathrm{H}-4.9958790 .2829671 .286179$

H -4.987438 -1.404567 0.765433

C $-6.535773-0.200809-0.147921$

H $-6.696449-0.944762-0.949226$

H -7.276889 -0.425737 0.636018

C $-6.7597131 .198698-0.709412$ 
$\mathrm{H}-6.7196511 .9305880 .117791$

$\mathrm{H}-7.7690001 .278723-1.144265$

C -5.699441 $1.560727-1.743870$

$\mathrm{H}-5.8502622 .590806-2.104784$

H -5.807025 $0.903892-2.626388$

C -4.293404 $1.410835-1.172280$

H -3.528769 $1.663078-1.920536$

$\mathrm{H}-4.1655842 .129250-0.349137$

C - 1.9207690 .3889791 .446066

$\mathrm{H}-0.8032160 .3915401 .402419$

C -2.315119 -0.346929 2.728181

H -2.025136 -1.405076 2.694559

$\mathrm{H}-3.412326-0.3234902 .850149$

C -1.6441620.321916 3.926506

$\mathrm{H}-1.922467-0.2046604 .853606$

H -0.549953 0.2123393.814502

C -2.000032 1.8006134 .027475

$\mathrm{H}-3.0754241 .8999344 .263837$

$\mathrm{H}-1.4557402 .2675254 .864548$

C -1.703621 2.5367332.726409

$\mathrm{H}-0.6146172 .5674192 .558773$

H -2.041203 3.5838822 .788699

C -2.356752 1.8516181 .533570

$\mathrm{H}-3.4539071 .8938721 .648555$

$\mathrm{H}-2.1088292 .3790940 .603831$

C -2.397970 -2.2299810.191796

$\mathrm{H}-3.106076-2.3862641 .025756$

C -2.892698 -3.014701-1.027492

H -3.920413 -2.720477-1.292729

H -2.258897 -2.759609-1.894856

C $-2.845578-4.521777-0.792161$

$\mathrm{H}-3.176308-5.050097-1.701091$

H $-3.568900-4.7851470 .000930$

C - $1.456397-4.987009-0.375404$

$\mathrm{H}-1.455550-6.072381-0.184970$

H - $0.749678-4.814330-1.207240$

C -0.977599-4.224183 0.852564

H $0.045151-4.5295921 .126326$

$\mathrm{H}-1.619598-4.4793821 .715641$

C -1.013569 -2.719370 0.616867

$\mathrm{H}-0.293104-2.453598-0.169964$

H $-0.666018-2.1882401 .513115$

C $1.353696-0.522886-2.468349$

H $0.827082-0.290969-3.412112$

C $2.816211-0.103867-2.631097$

H $3.340734-0.221657-1.671802$

H $2.8863840 .961789-2.894799$

C $3.527719-0.931280-3.697893$

H $3.089575-0.710756-4.689272$

H $4.586503-0.629646-3.748635$

C $3.414088-2.425362-3.424329$

H $3.940237-2.657180-2.481528$

H $3.915246-3.004231-4.217510$

C $1.954352-2.841667-3.298267$

H $1.447309-2.692404-4.269375$

H $1.873945-3.917382-3.068182$ 
C $1.237660-2.026881-2.227805$ H $0.175255-2.313093-2.181191$ H $1.671008-2.256806-1.237989$ C $0.8745092 .178096-1.576482$ H $1.9736582 .201651-1.451620$ C $0.2870673 .160076-0.569129$ $\mathrm{H}-0.8128003 .131483-0.657865$ H 0.5324872 .8325180 .454851 C $0.7766074 .582565-0.815869$ H $1.8667434 .623426-0.638219$ H $0.3175735 .274957-0.090667$ C $0.4803295 .032313-2.242473$ H $0.8865886 .041054-2.423173$ H -0.614532 5.110117-2.374155 C $1.0324794 .043104-3.262990$ H $0.7641264 .356339-4.285559$ H $2.1367134 .048792-3.216216$ C $0.5326692 .624843-2.996687$ H $0.9505351 .932212-3.744128$ H $-0.5641922 .600307-3.119362$ C $3.298263-0.1105630 .950668$ C $3.868286-1.3580090 .625444$ C 4.0454411 .0493510 .658720 C $5.120863-1.4347560 .019218$ H $3.317613-2.2806970 .814598$ C 5.2931840 .9587410 .045373 H 3.6333352 .0362760 .877122 C $5.845357-0.280789-0.280692$ H $5.528721-2.418176-0.231649$ H $5.8367381 .878634-0.188261$ H $6.824073-0.346231-0.760327$ N $2.407018-0.1016272 .740192$ C $2.716013-1.3717953 .355423$ H 3.729629 -1.729694 3.067687 H $2.757324-1.2170094 .449065$ C 2.9698551 .0371073 .432526 H 4.0185621 .2281593 .115548 H 3.0281310 .7851174 .506924 C $1.697906-2.4555883 .061008$ H $0.707944-2.1775013 .454885$ H $1.586634-2.6138261 .977889$ H $1.999482-3.4110993 .518557$ C 2.1584772 .3090513 .281515 H 1.9976032 .5563342 .220798 H 1.1678232 .1951823 .745566 H 2.670382 3.159400 3.759227

$$
\begin{aligned}
& \text { L1-Pd-(ArNR } 2) \\
& E=-2508.30691842 \\
& \text { Pd } 1.3842520 .3178640 .591414 \\
& P-2.412483-0.648750-0.060304 \\
& P-0.0234721 .047185-0.988286 \\
& C-1.6893730 .435660-1.175006 \\
& C-2.4062650 .739113-2.475924
\end{aligned}
$$


H -2.351874 $1.813159-2.732553$

$\mathrm{H}-3.4794130 .492840-2.448317$

H $-1.9981220 .195732-3.350362$

C -4.251131-0.403021-0.091647

$\mathrm{H}-4.517710-0.691834-1.124945$

C -5.059696 -1.305426 0.842334

$\mathrm{H}-4.840140-1.0375301 .889526$

H -4.775398 -2.361950 0.722255

C -6.556248 -1.1371870.590837

H -6.791580 -1.496379 -0.427529

H -7.127807 -1.775307 1.284271

C -6.9894280 .3183410 .725122$

$\mathrm{H}-6.8645200 .6356291 .776461$

H -8.062429 0.421956 0.496157

C $-6.1593831 .230719-0.171201$

H -6.448480 2.283590 -0.022126

H -6.373826 $0.997566-1.230226$

C -4.6656601 .0598160 .084042$

$\mathrm{H}-4.0744901 .704811-0.580416$

$\mathrm{H}-4.4326641 .3851731 .110663$

C - $1.719941-0.4820171 .630676$

$\mathrm{H}-0.622937-0.5249531 .396036$

C -2.063400 -1.586534 2.632077

$\mathrm{H}-1.926010-2.5884742 .201170$

H -3.126007 -1.515538 2.919713

C - $1.199625-1.4473383 .883413$

$\mathrm{H}-1.473297-2.2237634 .616516$

$\mathrm{H}-0.150578-1.6380733 .604300$

C -1.311829-0.0617234.510139

$\mathrm{H}-2.3297830 .0657814 .922328$

H -0.621217 0.026054 5.365332

C - 1.0479831 .0397443 .490236

H 0.0012060 .9972573 .140910

$\mathrm{H}-1.1907932 .0322393 .947776$

C - 1.9422840 .8883862 .266627

$\mathrm{H}-2.9993800 .9994932 .566768$

$\mathrm{H}-1.7275961 .6757971 .533706$

C -2.225684 -2.467294 -0.450361

$\mathrm{H}-2.841375-3.0451440 .262608$

C -2.731345 -2.741924-1.870293

$\mathrm{H}-3.811277-2.535071-1.941810$

H - $2.233724-2.041865-2.562682$

C $-2.453385-4.171901-2.321218$

$\mathrm{H}-2.803520-4.309339-3.357228$

H -3.038968 $-4.872984-1.698777$

C - $0.974230-4.513791-2.202664$

$\mathrm{H}-0.790749-5.552270-2.522743$

H $-0.394721-3.867286-2.886079$

C $-0.489651-4.300362-0.775146$

H $0.588432-4.515147-0.693341$

$\mathrm{H}-1.001548-5.020810-0.110688$

C $-0.761018-2.881108-0.288546$

$\mathrm{H}-0.126492-2.166251-0.836021$

$\mathrm{H}-0.457178-2.7929990 .763607$

C $0.7362470 .716488-2.680024$

H $0.0547081 .168279-3.424984$ 
C $2.1133211 .357035-2.857391$

H $2.7683291 .023532-2.031584$

H $2.0409312 .451366-2.769049$

C $2.7473041 .004101-4.200803$

H 2.153949 $1.461190-5.013932$

H $3.7551091 .446681-4.266926$

C $2.809888-0.501603-4.422956$

H $3.483541-0.950537-3.669865$

H $3.247127-0.730967-5.408664$

C $1.426390-1.124740-4.287229$

H $0.778010-0.747074-5.099039$

H $1.478154-2.219271-4.415438$

C $0.799339-0.785001-2.940977$

H - $0.211791-1.212220-2.866201$

H $1.388604-1.253163-2.130801$

C - $0.1653432 .933584-1.107849$

$\mathrm{H}-0.1584463 .172761-2.189104$

C $1.0299403 .630635-0.454802$

H 1.0253443 .3735850 .619984

H $1.9781873 .234285-0.845403$

C $0.9729825 .144951-0.632715$

H $1.0711305 .387102-1.707044$

H $1.8324715 .618646-0.129569$

C $-0.3355855 .722564-0.105777$

$\mathrm{H}-0.3865426 .807455-0.296159$

H -0.366532 5.5975320 .992179

C -1.538509 5.012574-0.717110

$\mathrm{H}-2.4750215 .401064-0.282861$

$\mathrm{H}-1.5826875 .234405-1.799233$

C - $1.4539123 .501961-0.520161$

$\mathrm{H}-2.3320272 .999235-0.948789$

$\mathrm{H}-1.4777923 .2853470 .561777$

C $4.244240-0.7514951 .237899$

C $4.085036-1.049058-0.114038$

C $5.476109-0.9934911 .853410$

C $5.143815-1.578717-0.849452$

H $3.102753-0.839238-0.560938$

C $6.535741-1.5192241 .117034$

H $5.615983-0.7676962 .913166$

C $6.373414-1.813300-0.236791$

H $5.004238-1.803880-1.909653$

H $7.496503-1.6997741 .605339$

H $7.206400-2.223797-0.812407$

N $3.121259-0.2149651 .984918$

C $2.649885-1.1926992 .982342$

H $3.446107-1.3836233 .730940$

H $1.817565-0.7132073 .520181$

C 3.4622381 .0550882 .656744

H 4.2149490 .8771493 .452004

H 2.5433351 .3926843 .160991

C 3.9463862 .1207981 .698624

H 4.0908683 .0708072 .233838

H 4.9002301 .8419291 .227844

H 3.1980352 .2675850 .903654

C $2.191523-2.4937262 .362539$

H $1.431734-2.2885181 .591441$ 
H $3.023333-3.0307991 .884415$

H $1.756734-3.1497323 .130911$

Piperidine

L1-Pd(Ar)-Cl(HNR2)

$E=-2546.36334135$

Pd $1.2503920 .720883-0.225088$

P -2.304952 -0.054035 0.393418

P $0.400737-1.435479-0.026739$

C - $1.347776-1.4865150 .323758$

C $-1.916638-2.7419280 .967535$

$\mathrm{H}-1.564205-2.9149952 .002488$

$\mathrm{H}-1.662564-3.6486070 .396333$

H -3.014908 -2.729156 1.028079

C - $4.087152-0.5258390 .224715$

$\mathrm{H}-4.267942-1.1170401 .140702$

C -5.072690 0.6448910 .253315

$\mathrm{H}-4.9423351 .256181-0.655804$

$\mathrm{H}-4.8782531 .3096671 .108737$

C -6.509997 0.1324340.311782

H -6.665375 -0.386176 1.275221

$\mathrm{H}-7.2109470 .9826030 .301583$

C -6.817644 -0.826815 -0.832469

$\mathrm{H}-6.784818-0.271598-1.787473$

$\mathrm{H}-7.843267-1.218235-0.737797$

C $-5.810331-1.970140-0.888890$

H -6.017295 -2.622757 -1.752165

H -5.919603 -2.602464 0.011151

C -4.379970 -1.446338 -0.964591

H -3.653831 -2.270495-1.001755

H -4.254404 -0.885410 -1.902867

C -1.848769 $1.187950-0.891277$

$\mathrm{H}-0.7332541 .166857-0.859284$

C -2.268014 2.639903 -0.644624

$\mathrm{H}-2.0102472 .9698470 .370180$

H -3.363789 2.729202 -0.738933

C - $1.5720033 .550643-1.653058$

$\mathrm{H}-1.8865694 .594171-1.489851$

$\mathrm{H}-0.4900803 .504741-1.441260$

C - $1.8391613 .134664-3.094209$

$\mathrm{H}-2.9056623 .299717-3.335755$

$\mathrm{H}-1.2628913 .771847-3.784398$

C -1.502061 1.665611 -3.322144

H $-0.4134481 .513405-3.214188$

H -1.763489 $1.360742-4.348328$

C -2.222825 $0.777660-2.314976$

$\mathrm{H}-3.3124970 .894897-2.448626$

$\mathrm{H}-1.989968-0.281808-2.480941$

C -2.2571200.901208 1.994923

H -2.959579 1.7488831 .902003

C -2.708167 0.0150403 .160272

$\mathrm{H}-3.748906-0.3168423 .017541$

H -2.087827 -0.897688 3.172240 
C -2.582623 0.7290474 .502724

$\mathrm{H}-2.8847060 .0482755 .314988$

H -3.290197 1.5776134 .528533

C - 1.1698151 .2490844 .733924

$\mathrm{H}-1.1087511 .7814375 .696708$

$\mathrm{H}-0.4735150 .3939064 .804246$

C - 0.7350642 .1563493 .590970

H 0.3021142 .4983103 .737361

$\mathrm{H}-1.3639023 .0653713 .585990$

C - 0.8508831 .4528802 .243426

$\mathrm{H}-0.1319220 .6187382 .205451$

H -0.546962 2.141710 1.440214

C $1.173777-2.4830631 .315407$

H $0.521812-3.3683671 .422491$

C $2.583938-2.9933221 .005634$

H $3.248970-2.1458750 .783849$

H $2.574418-3.6273920 .106636$

C $3.158918-3.7895842 .174122$

H $2.569463-4.7145022 .316296$

H $4.185692-4.1080701 .931605$

C $3.141912-2.9854393 .467796$

H $3.799396-2.1049293 .352759$

H $3.553229-3.5801354 .299778$

C $1.728798-2.5175763 .791108$

H $1.090976-3.3983293 .990357$

H $1.718693-1.9117664 .712604$

C $1.135596-1.7173272 .637899$

H $0.097184-1.4313032 .866781$

H $1.698824-0.7754352 .514892$

C $0.800036-2.422681-1.552733$

H $1.903446-2.478720-1.518162$

C $0.429721-1.669983-2.826549$

H -0.668711 -1.585292 -2.877087

H $0.822706-0.639960-2.784095$

C $0.929303-2.398262-4.069287$

H $2.034446-2.413585-4.059036$

H $0.634355-1.847864-4.977923$

C $0.404394-3.829147-4.119263$

H $0.809468-4.359999-4.996251$

H -0.692935 -3.804163 -4.250836

C $0.733068-4.587978-2.838295$

H $0.300173-5.601481-2.870278$

H $1.827798-4.719992-2.764056$

C $0.239774-3.842635-1.600081$

H $0.507839-4.405065-0.691362$

H - $0.862831-3.784235-1.624382$

C $3.1392790 .162444-0.260463$

C 3.9357270 .3268210 .879743

C $3.721504-0.380112-1.412228$

C $5.271017-0.0801050 .881207$

H 3.5131360 .7681171 .786268

C $5.058670-0.783768-1.409884$

H $3.131262-0.499049-2.324300$

C $5.837001-0.645488-0.261354$

H 5.8724480 .0462271 .786023

H $5.493656-1.211636-2.317649$ 
H $6.881132-0.967248-0.259565$

C 2.2427593 .3303820 .739531

C $2.3706533 .084882-1.623044$

C 2.0676224 .8457910 .636216

H 3.3309303 .1187350 .866129

H 1.7448842 .9599021 .651438

C $2.2308284 .591740-1.848022$

H $3.4569982 .836746-1.594033$

H $1.9529172 .539244-2.485793$

C $2.7281015 .366710-0.634104$

H 2.4914895.3362101.528942

H 0.9878815 .0777090 .617510

H $2.7851644 .886258-2.755074$

H $1.1690154 .833268-2.028201$

H $2.5528516 .448530-0.757409$

H $3.8220695 .234503-0.546638$

N $1.7012222 .654806-0.415068$

TS (reductive elimination)

$E=-2546.34128272$

Pd $1.1950660 .561507-0.390065$

P -2.421290 0.2469870.441404

P $0.072650-1.437318-0.121523$

C -1.656006 -1.289166 0.282870

C - $2.380409-2.4977980 .853341$

$\mathrm{H}-2.353975-3.3638180 .172482$

$\mathrm{H}-3.445617-2.3037201 .050013$

$\mathrm{H}-1.962175-2.8466421 .816722$

C -4.245566 0.0319630 .213110

H -4.542147 -0.573449 1.088830

C -5.056710 1.328239 0.272187

$\mathrm{H}-4.7967281 .961860-0.592796$

H -4.8109231 .9110341 .173239$

C -6.553288 1.0275650 .238973

$\mathrm{H}-6.8295130 .4821731 .159568$

$\mathrm{H}-7.1250661 .9694670 .253724$

C -6.934469 $0.190292-0.976714$

$\mathrm{H}-6.7706080 .787749-1.891967$

H -8.009414 -0.050725 -0.953372

C $-6.101150-1.083894-1.059389$

H $-6.353845-1.650492-1.969970$

$\mathrm{H}-6.349534-1.741380-0.206296$

C -4.607589-0.776585-1.036408

H -4.010551-1.698135-1.081046

H -4.344685 -0.197904-1.935547

C - $1.7730971 .505763-0.736082$

$\mathrm{H}-0.6716831 .348033-0.610367$

C -2.013896 2.984643 -0.423007

$\mathrm{H}-1.8283043 .2163170 .633999$

H -3.068682 $3.240718-0.621215$

C -1.102314 3.841570-1.300676

$\mathrm{H}-1.2714734 .908882-1.085842$

H $-0.0539473 .621918-1.030653$

C -1.308662 3.557381-2.784338 
$\mathrm{H}-2.3187313 .894430-3.082131$

$\mathrm{H}-0.5958864 .143932-3.386047$

C -1.166863 2.071382 -3.093089

$\mathrm{H}-0.1219001 .756942-2.917074$

$\mathrm{H}-1.3841071 .873050-4.154946$

C -2.078612 1.230542 -2.206073

$\mathrm{H}-3.1314491 .492572-2.412790$

$\mathrm{H}-1.9610030 .163084-2.426917$

C -2.276709 1.0772012 .106484

$\mathrm{H}-2.8782972 .0038272 .078411$

C -2.8349970.164065 3.202692

H -3.907081 -0.0295873 .038246$

$\mathrm{H}-2.327661-0.8139543 .140467$

C -2.631390 0.7498394.596488

$\mathrm{H}-3.0167710 .0482865 .353998$

H -3.231390 1.6729704 .693382

C - 1.1670081 .0750454 .860373

$\mathrm{H}-1.0454301 .5168285 .862494$

$\mathrm{H}-0.5794330 .1392814 .857047$

C - 0.6225252 .0127383 .791221

H 0.4480242 .2141103 .959330

$\mathrm{H}-1.1375112 .9881473 .865901$

C -0.8161841.440705 2.391482

$\mathrm{H}-0.2002860 .5364912 .275109$

H -0.4310842.150227 1.644205

C $0.795803-2.5729591 .174052$

H $0.133107-3.4564091 .229023$

C $2.205931-3.0571570 .827132$

H $2.860010-2.1867230 .661945$

H $2.199899-3.625762-0.114840$

C $2.792850-3.9293301 .933809$

H 2.212976 -4.8681982.008366

H $3.822477-4.2175431 .666667$

C $2.773106-3.2231063 .283377$

H $3.435399-2.3408203 .234628$

H $3.177798-3.8803624 .070497$

C $1.362124-2.7699553 .636128$

H $0.716660-3.6568373 .774860$

H $1.355775-2.2279784 .596860$

C $0.777393-1.8886132 .538893$

H - $0.252484-1.5930112 .791649$

H $1.365439-0.9567412 .467543$

C $0.303573-2.469111-1.663092$

H $1.405502-2.546469-1.725705$

C $-0.160763-1.726984-2.911575$

$\mathrm{H}-1.258385-1.615026-2.862417$

H $0.266419-0.710073-2.920263$

C $0.210534-2.479984-4.184744$

H $1.311470-2.509778-4.276485$

H -0.160095 -1.936709 -5.069848

C - $0.332711-3.904495-4.168599$

H $-0.012838-4.451292-5.070763$

$\mathrm{H}-1.437110-3.868617-4.202379$

C $0.100182-4.649132-2.910581$

H - $0.341617-5.659119-2.890208$

H $1.196286-4.789756-2.928620$ 
C $-0.280618-3.880933-1.646967$

H $0.047021-4.437389-0.754871$

$\mathrm{H}-1.380420-3.808879-1.588702$

C $3.1719520 .335711-0.107919$

C 3.6774120 .3156891 .204875

C $3.911362-0.307854-1.117196$

C $4.880144-0.3257341 .492725$

H 3.1201910 .7952732 .012906

C $5.111120-0.949501-0.816125$

H $3.536346-0.324939-2.143140$

C $5.609333-0.9647720 .488100$

H $5.247069-0.3319802 .523207$

H $5.659641-1.454469-1.616409$

H $6.548782-1.4710520 .719041$

C $2.8774602 .550856-1.932780$

C 2.6238553 .1297970 .406497

C $4.3231153 .043480-1.934271$

H $2.2234463 .361181-2.331416$

H $2.7575831 .707524-2.630418$

C 4.0617033 .6404280 .474312

H 1.9464524 .0002730 .242534

H 2.3199542 .7020471 .374490

C $4.5152014 .137725-0.892190$

H $4.5904533 .405355-2.941301$

H $4.9881582 .194418-1.703511$

H 4.1359284 .4398211 .230708

H 4.7175212 .8165420 .801665

H $5.5656994 .469836-0.860209$

H $3.9152825 .023180-1.176868$

N $2.4062772 .155751-0.630783$

\section{L1-Pd-(ArNR $)$}

$E=-2546.39746986$

Pd $1.366603-0.611813-0.437732$

P - $2.3535760 .662348-0.127965$

P $0.051122-0.4962231 .367207$

C - 1.5837250 .2119881 .336368

C -2.201063 0.6337982 .654905

$\mathrm{H}-2.166612-0.1799683 .402697$

H -3.262463 0.9152892 .566768

$\mathrm{H}-1.6996911 .5017053 .125370$

C -4.191178 0.5796340 .106741

$\mathrm{H}-4.3843621 .3667190 .858701$

C -5.025266 $0.926357-1.128070$

$\mathrm{H}-4.8689130 .154945-1.901108$

H -4.705585 $1.882469-1.570066$

C -6.510219 $0.985627-0.777944$

H -6.680525 $1.823726-0.077936$

H -7.100367 $1.211346-1.681001$

C $-6.990118-0.311760-0.137235$

$\mathrm{H}-6.930832-1.125296-0.883053$

H $-8.051703-0.2293250 .146906$

C $-6.138488-0.6832011 .071726$

H -6.465494 -1.648390 1.491016 
$\mathrm{H}-6.2872640 .0675471 .869289$

C -4.657196 -0.745918 0.714986

$\mathrm{H}-4.047645-0.9960681 .594106$

H -4.493017 - $1.558911-0.010081$

C - $1.801060-0.357868-1.548338$

$\mathrm{H}-0.688501-0.302508-1.399518$

C -2.130946 $0.147792-2.953812$

$\mathrm{H}-1.9261051 .221456-3.066670$

$\mathrm{H}-3.2064650 .013710-3.160781$

C -1.319680 -0.637364 -3.981185

$\mathrm{H}-1.565510-0.294784-4.999685$

$\mathrm{H}-0.250524-0.411318-3.824380$

C -1.545509 -2.141663 -3.861630

$\mathrm{H}-2.579716-2.372518-4.176886$

H - $0.884279-2.681516-4.559743$

C - $1.338921-2.641998-2.435660$

$\mathrm{H}-0.276617-2.545534-2.145009$

$\mathrm{H}-1.591240-3.712624-2.365249$

C $-2.158432-1.837865-1.435084$

$\mathrm{H}-3.235120-1.981090-1.637008$

$\mathrm{H}-1.970404-2.190396-0.413162$

C -2.078020 2.418498-0.701605

H -2.729092 2.603647 -1.575397

C -2.461517 3.3974230 .412590

$\mathrm{H}-3.5326783 .3085260 .655088$

$\mathrm{H}-1.9109053 .1195731 .327361$

C -2.138750 4.8434460.049870

$\mathrm{H}-2.3927195 .5044630 .894440$

H -2.777664 5.158554 -0.795263

C $-0.6761905 .009750-0.341835$

$\mathrm{H}-0.4693726 .055054-0.623371$

$\mathrm{H}-0.0359244 .7855360 .530390$

C $-0.3169004 .065231-1.480577$

H $0.7470794 .167773-1.748132$

$\mathrm{H}-0.8901664 .349461-2.382373$

C - $0.6190742 .614705-1.122232$

H $0.0356622 .284188-0.301005$

H $-0.3696411 .961562-1.971260$

C 0.9587280 .5566622 .634617

H 0.3469070 .5424693 .556575

C 2.3447470 .0107812 .977499

H $2.922658-0.0731862 .037676$

H $2.269499-1.0055243 .393397$

C 3.0854930 .9084303 .965605

H 2.5549160 .8964014 .935685

H 4.0922430 .5014084 .159161

C 3.1804472 .3437703 .463882

H 3.7993142 .3654582 .550011

H 3.6880352 .9811424 .207038

C 1.7997772 .8968933 .133999

H 1.2061552 .9813294 .063105

H 1.8822653 .9177432 .723480

C 1.0683301 .9971332 .145044

H 0.0640012 .3936051 .932073

H 1.6138961 .9901861 .183766

C $-0.083288-2.1003962 .363238$ 
H $0.054171-1.8155303 .424402$

C $1.027057-3.0829601 .981296$

H $0.896222-3.3428230 .915011$

H $2.014505-2.6013322 .040365$

C $0.990138-4.3444432 .838746$

H $1.214562-4.0763863 .887678$

H $1.782747-5.0430642 .522141$

C $-0.372859-5.0243752 .775425$

H -0.401707 -5.900897 3.443815

H - $0.534718-5.4084251 .751444$

C -1.493637 -4.049526 3.119704

H -2.475430 -4.5410913.014707

$\mathrm{H}-1.406661-3.7536434 .181305$

C -1.434370 -2.799766 2.246318

$\mathrm{H}-2.253547-2.1094252 .492235$

H -1.592621 -3.095951 1.195159

C $3.7416900 .684190-1.145805$

C $3.0540821 .882731-1.411435$

C $4.8980770 .745697-0.358210$

C $3.5005403 .088825-0.891250$

H $2.1325141 .853669-1.994549$

C 5.3337481 .9631890 .169642

H $5.468246-0.154301-0.135440$

C $4.6446153 .140925-0.090319$

H $2.9364134 .001050-1.098766$

H 6.2319501 .9793240 .791810

H 4.9868814 .0899790 .327067

C $3.078507-0.510594-3.152126$

C $3.991070-1.744961-1.286156$

C $2.366058-1.746675-3.666677$

H $4.080153-0.419998-3.622067$

H $2.5126230 .389633-3.420420$

C $3.295450-3.015073-1.742763$

H $5.018395-1.704557-1.707063$

H $4.069043-1.741648-0.190704$

C $3.081497-3.021769-3.248370$

H $2.275385-1.677683-4.762383$

H $1.345957-1.749581-3.251461$

H $3.894354-3.881666-1.420823$

H $2.323101-3.070514-1.220530$

H $2.505076-3.908347-3.556689$

H $4.057550-3.085293-3.762573$

N $3.242930-0.547109-1.687982$

$\mathrm{R}=t \mathrm{Bu}$

$$
\text { L1-Pd(Ar)-Cl( } \left.\mathrm{H}_{2} \mathrm{NR}\right)
$$

$E=-2508.29123032$

Pd -1.405238 -0.510153 -0.635084

P $2.263894-0.2570210 .005722$

P -0.4255150 .5071521 .227383$

C 1.3422910 .3505391 .329852

C 2.0072630 .5073742 .686473

H 2.0507251 .5551143 .035311 
H 3.0430860 .1369672 .700778

H $1.489512-0.0616023 .478655$

C 4.0458500 .1600050 .276679

H $4.289984-0.4158461 .187590$

C $4.984389-0.336467-0.826654$

H $4.7838070 .221491-1.757259$

H $4.803941-1.398145-1.053924$

C $6.442815-0.135481-0.421975$

H $6.666100-0.7873640 .442085$

H $7.106507-0.465040-1.237548$

C $6.7345281 .313226-0.047946$

H $6.6307941 .946526-0.947709$

H 7.7793851 .4188600 .285561

C 5.7754131 .8158151 .025517

H 5.9694392 .8773321 .248050

H 5.9535911 .2638811 .966498

C 4.3218151 .6320220 .601215

H 3.6360231 .9911931 .380152

H $4.1298582 .250205-0.287707$

C $1.6828140 .374343-1.628893$

H $0.5777220 .363205-1.481810$

C $1.982272-0.488788-2.857771$

H $1.678919-1.531933-2.701185$

H $3.067763-0.497647-3.058581$

C $1.2410790 .065238-4.072399$

H $1.461248-0.552558-4.957726$

H $0.156443-0.032353-3.888508$

C $1.5887911 .524478-4.341190$

H $2.6494001 .591591-4.645681$

H $0.9994321 .909018-5.189274$

C $1.3707492 .388590-3.104823$

H $0.2938122 .449657-2.875312$

H $1.7055303 .421635-3.292589$

C $2.1028591 .821736-1.894482$

H $3.1880401 .851757-2.094680$

H $1.9205432 .443035-1.008055$

C $2.284041-2.116018-0.174260$

H $2.902254-2.365496-1.054772$

C $2.900589-2.7653271 .068382$

H $3.957864-2.4749511 .174633$

H 2.377680 -2.380295 1.962000

C $2.798478-4.2876371 .038088$

H $3.218320-4.7048641 .967815$

H $3.424387-4.6747110 .213583$

C $1.363695-4.7570200 .835899$

H $1.321746-5.8573420 .794598$

H $0.753549-4.4526871 .705475$

C $0.774745-4.148598-0.428682$

$\mathrm{H}-0.277058-4.447199-0.561503$

H $1.318773-4.534996-1.309574$

C $0.863101-2.627902-0.410881$

H $0.221474-2.2484060 .404990$

H $0.433367-2.234904-1.344191$

C $-1.103310-0.3172222 .762258$

$\mathrm{H}-0.6354500 .1828603 .629585$

C -2.619027 -0.160066 2.885344 
H -3.088319 -0.629156 2.002267

$\mathrm{H}-2.9093640 .9015002 .868159$

C -3.150564 -0.824637 4.152153

$\mathrm{H}-2.747307-0.2954515 .034831$

H -4.246573 -0.717863 4.200865

C - $2.755227-2.2949714 .222554$

$\mathrm{H}-3.255313-2.8423233 .402864$

H -3.112281 -2.746882 5.162225

C - $1.246925-2.4653824 .084305$

$\mathrm{H}-0.744970-2.0178504 .961495$

$\mathrm{H}-0.977209-3.5345654 .083821$

C $-0.730125-1.7964772 .815801$

H $0.360430-1.9150982 .727864$

$\mathrm{H}-1.171710-2.3025541 .938078$

C - 0.9293352 .2802381 .486725

$\mathrm{H}-2.0180782 .2624131 .301705$

C -0.308892 3.160859 0.407364

H 0.7849283 .1762470 .560469

H -0.491697 2.720268 -0.584198

C -0.861229 4.580120 0.461551

$\mathrm{H}-1.9366694 .5513070 .211188$

H $-0.3797205 .204089-0.309523$

C -0.6682075.196872 1.842370

$\mathrm{H}-1.1191606 .2018851 .885266$

H 0.4130585 .3334372 .028535

C -1.2498194.307247 2.935865

$\mathrm{H}-1.0586624 .7442143 .930016$

$\mathrm{H}-2.3480954 .2627422 .820981$

C - 0.6900102 .8874512 .868702

$\mathrm{H}-1.1473152 .2726553 .659548$

H 0.3931552 .9123263 .077725

C $-2.6393180 .963079-1.068290$

C $-3.7413751 .300984-0.271715$

C -2.427232 $1.699093-2.242369$

C -4.578466 2.364146-0.617708

$\mathrm{H}-3.9589750 .7342000 .634923$

C -3.258406 2.767615 -2.583849

$\mathrm{H}-1.6020191 .439511-2.909482$

C -4.336538 $3.110824-1.769622$

H -5.4295392.607910 0.024561

$\mathrm{H}-3.0617533 .330732-3.500649$

H $-4.9892103 .945343-2.036523$

$\mathrm{N}-2.002980-1.670304-2.132245$

C $-3.208838-2.484492-2.064120$

C $-3.373810-3.013487-0.639006$

C $-4.466373-1.699625-2.461616$

C $-3.033053-3.671825-3.022030$

$\mathrm{H}-2.472556-3.564340-0.326308$

H -3.519729 -2.177007 0.063419

H -4.238721 -3.690196 -0.558641

$\mathrm{H}-4.363781-1.303849-3.485227$

H -5.369512 -2.331418 -2.433208

H -4.612732 -0.842152 -1.789447

H -3.912819 -4.336231 -3.013131

$\mathrm{H}-2.890037-3.314978-4.054823$

H -2.144317 -4.257435 -2.741665 
H -1.984797 -1.213069 -3.045643

TS (reductive elimination)

$E=-2508.26668972$

$\mathrm{Pd}-1.348924-0.468305-0.639368$

P $2.354217-0.197551-0.054254$

P -0.3229640 .3079971 .271720$

C 1.4531120 .2675281 .338329

C 2.1464050 .4189372 .679523

H 2.195143 1.4644493 .037049

H 3.1819710 .0455532 .675753

H $1.637659-0.1543653 .473976$

C 4.1290860 .2535960 .204912

H $4.412095-0.3694541 .072797$

C $5.054510-0.137933-0.950376$

H $4.8088780 .471359-1.837107$

H $4.904619-1.188417-1.242142$

C $6.5160690 .087638-0.570741$

H $6.783971-0.6095900 .243843$

H $7.167719-0.166692-1.422223$

C $6.7705851 .518721-0.111712$

H $6.6231622 .203864-0.966269$

H 7.8197861 .6383770 .203018

C 5.8233951 .9191141 .013897

H 5.9875372 .9707291 .298818

H 6.0438111 .3158041 .913451

C 4.3663631 .7117630 .613083

H 3.6902432 .0014481 .428166

H $4.1326922 .374683-0.232634$

C $1.7071340 .542220-1.617508$

H $0.6075010 .454921-1.440525$

C $2.009366-0.186744-2.929326$

H $1.787389-1.259538-2.858046$

H $3.082796-0.100922-3.173500$

C $1.1762430 .419981-4.056711$

H $1.391853-0.099631-5.004309$

H $0.1100560 .234512-3.833326$

C $1.4180311 .917536-4.204662$

H $2.4561382 .082178-4.547209$

H $0.7649832 .335819-4.987761$

C $1.2076562 .651842-2.885880$

H $0.1416352 .616408-2.604134$

H $1.4642363 .718258-2.992820$

C $2.0319672 .029346-1.765301$

H $3.1026882 .144782-2.009439$

H $1.8568152 .556437-0.818363$

C $2.428802-2.032069-0.408767$

H $3.031713-2.172739-1.323484$

C $3.103714-2.7732240 .748386$

H $4.149373-2.4460350 .861886$

H $2.586012-2.5030821 .685998$

C $3.068769-4.2877260 .563797$

H $3.527074-4.7783571 .437843$

H $3.692756-4.559229-0.307101$ 
C $1.652373-4.8014840 .340740$

H $1.659301-5.8922440 .184508$

H $1.050573-4.6183741 .249075$

C $1.003001-4.093215-0.840446$

H - $0.037410-4.431118-0.976191$

H $1.540822-4.359158-1.768777$

C $1.022298-2.580949-0.655732$

H $0.394259-2.3191810 .211508$

H $0.549790-2.093464-1.523997$

C $-0.928775-0.6599742 .750698$

H $-0.509763-0.1784873 .652992$

C -2.454240 -0.626706 2.852999

$\mathrm{H}-2.865942-1.0727141 .930125$

H -2.828044 0.4078362 .885406

C -2.954252 -1.4031944.067401

H -2.610294 -0.897099 4.987987

$\mathrm{H}-4.056133-1.3856654 .098364$

C -2.445282 -2.839959 4.063705

H -2.889269 -3.376700 3.205385

H -2.779113 -3.371730 4.969666

C $-0.926140-2.8857253 .944066$

$\mathrm{H}-0.473585-2.4522204 .854493$

$\mathrm{H}-0.574601-3.9296163 .889581$

C - $0.444495-2.1067332 .725169$

H $0.653200-2.1387612 .648036$

H -0.837321 -2.591048 1.812679

C -0.9556612 .0196711 .665814$

$\mathrm{H}-2.0397131 .9308871 .464355$

C - 0.3955133 .0218240 .662710

H 0.6962643 .0936960 .816227

H $-0.5562132 .647180-0.359815$

C - 1.0330784 .3962600 .825005

$\mathrm{H}-2.1045374 .3215290 .567963$

H -0.5893705 .1069220 .107736$

C - 0.8801814 .9133722 .250812

$\mathrm{H}-1.3895875 .8840072 .368434$

H 0.1905145 .0971392 .456796

C - 1.4141173 .9071553 .264312

$\mathrm{H}-1.2580214 .2750414 .292086$

H -2.5064993.8052543.131483

C -0.7677112.533744 3.092729

$\mathrm{H}-1.1933801 .8324203 .827256$

H 0.3102802 .6067523 .318400

C $-2.9122060 .713127-1.101800$

C $-4.0024520 .783660-0.212974$

C -2.672575 $1.829898-1.930826$

C $-4.7935531 .930703-0.144209$

$\mathrm{H}-4.225480-0.0518110 .450874$

C -3.467159 2.968579-1.853285

$\mathrm{H}-1.8455841 .802762-2.645947$

C - $4.5368073 .034555-0.956465$

$\mathrm{H}-5.6231341 .9578310 .568369$

$\mathrm{H}-3.2425493 .819508-2.502786$

H -5.159196 $3.929608-0.895435$

$\mathrm{N}-2.547118-0.932087-2.191949$

C $-3.546429-1.988129-2.272137$ 
$\begin{array}{lll}\text { C }-3.653901 & -2.707879 & -0.929377 \\ \text { C }-4.915350 & -1.434119 & -2.688159 \\ \text { C }-3.064800 & -2.991788 & -3.329435 \\ \text { H }-2.667379 & -3.085439 & -0.617515 \\ \text { H }-4.019579 & -2.035277 & -0.142092 \\ \text { H }-4.349717 & -3.557803 & -1.001584 \\ \text { H }-4.842071 & -0.926788 & -3.663726 \\ \text { H }-5.662924 & -2.237631 & -2.781913 \\ \text { H }-5.278963 & -0.699135 & -1.956659 \\ \text { H }-3.783664 & -3.818478 & -3.452853 \\ \text { H }-2.946637 & -2.499110 & -4.307955 \\ \text { H }-2.088891 & -3.409516 & -3.038871 \\ \text { H }-2.486401 & -0.420707 & -3.066838\end{array}$

L1-Pd-(ArNHR)

$E=-2508.32533534$

Pd $1.5022010 .260146-0.239495$

P - 2.267371 -0.342989 -0.458276

$\mathrm{P}-0.0843131 .2086341 .021410$

C - 1.7983030 .8068710 .734804

C -2.882940 1.6936981 .323339

$\mathrm{H}-2.9480751 .6340442 .423625$

$\mathrm{H}-3.8881671 .4461470 .949501$

$\mathrm{H}-2.7412062 .7633381 .080619$

C $-3.950438-0.989311-0.026755$

$\mathrm{H}-4.603800-0.106240-0.146295$

C $-4.485547-2.068943-0.969829$

H -3.866514 -2.977046 - 0.873537

H $-4.413058-1.749966-2.021110$

C $-5.933260-2.413715-0.628872$

$\mathrm{H}-6.567922-1.530305-0.824610$

H -6.297314 -3.210914 -1.297262

C -6.085017 -2.829642 0.829944

H -5.545687 -3.780805 0.991197

H -7.143272 -3.030622 1.062964

C $-5.519525-1.7724551 .772158$

H $-5.592338-2.1114272 .818210$

H $-6.129940-0.8536401 .700728$

C $-4.070669-1.4384351 .432592$

H -3.674558 -0.6636752 .103336$

H -3.446442 -2.330996 1.594135

C $-1.070946-1.720895-0.662225$

$\mathrm{H}-0.107030-1.136512-0.658402$

C $-1.131180-2.525902-1.962318$

$\mathrm{H}-1.194999-1.875611-2.845171$

$\mathrm{H}-2.033683-3.161202-1.977090$

C $0.107456-3.412654-2.075489$

H 0.066929 -3.999582 -3.007999

H $0.996930-2.761907-2.148004$

C $0.267055-4.332175-0.870901$

H $-0.559987-5.066368-0.867510$

H $1.201040-4.910534-0.953619$

C $0.247042-3.5439540 .432737$

H $1.137966-2.8941350 .484624$ 
H $0.303317-4.2262201 .296423$

C - $0.998991-2.6712830 .531813$

$\mathrm{H}-1.893950-3.3196800 .546323$

H $-0.996690-2.1014551 .468579$

C $-2.4946630 .323726-2.190350$

H - $2.871538-0.496544-2.828193$

C -3.522711 $1.459033-2.193381$

$\mathrm{H}-4.5111891 .089339-1.876818$

H -3.216087 2.212452 -1.447341

C -3.643473 2.125152 -3.560466

$\mathrm{H}-4.3684042 .953837-3.508045$

H -4.054242 $1.397690-4.284301$

C -2.294362 $2.622410-4.062598$

$\mathrm{H}-2.3990433 .081364-5.059198$

$\mathrm{H}-1.9254103 .416243-3.388320$

C -1.281784 $1.485482-4.097589$

$\mathrm{H}-0.2947731 .856070-4.420201$

$\mathrm{H}-1.5979350 .742000-4.852748$

C $-1.1500410 .808987-2.738168$

$\mathrm{H}-0.7101251 .509335-2.013085$

H $-0.428024-0.018280-2.798064$

C -0.0025603 .0812170 .959759$

H -0.747848 3.4786051 .672924

C 1.3758643 .6026851 .368475

H 2.1294993 .1230550 .716222

H 1.6198933 .2952292 .397073

C 1.4737825 .1215231 .253867

H 0.7951285 .5858011 .992863

H 2.4915025 .4540441 .518077

C $1.0988545 .609928-0.140068$

H $1.8425325 .234362-0.866796$

H $1.1419396 .710493-0.189796$

C -0.282735 5.105899 -0.539199

$\mathrm{H}-1.0419705 .5620450 .122312$

$\mathrm{H}-0.5310865 .427925-1.564671$

C $-0.3662903 .587949-0.432773$

$\mathrm{H}-1.3718573 .234678-0.707534$

H $0.3321853 .129518-1.156936$

C 0.3211680 .9211542 .834645

H 1.3738371 .2567652 .892922

C $0.314362-0.5676353 .164174$

H -0.724396 -0.935965 3.085566

H $0.904795-1.1122692 .408642$

C $0.842967-0.8416784 .567947$

H $1.911971-0.5624774 .610509$

H $0.795308-1.9211984 .789309$

C $0.075198-0.0486495 .619785$

H $0.502467-0.2165966 .622333$

H -0.965664 -0.419036 5.659556

C 0.0578281 .4393695 .287739

H -0.539770 1.9921876 .031749

H 1.0861351 .8394975 .356233

C -0.4816441 .6927583 .881388$

$\mathrm{H}-0.4787872 .7734493 .668336$

$\mathrm{H}-1.5346491 .3674763 .838797$

C $3.600636-1.802584-0.642132$ 
C $3.757752-1.7772660 .749730$

C $3.774603-3.010303-1.323682$

C $4.087552-2.9401381 .437827$

H $3.557571-0.8443331 .280951$

C $4.084846-4.178748-0.627633$

H $3.652293-3.037672-2.409941$

C $4.247334-4.1475190 .754659$

H $4.192919-2.9074152 .524774$

H $4.201437-5.117536-1.174361$

H $4.487753-5.0619451 .301376$

N $3.205100-0.620212-1.344847$

C $4.2693100 .376594-1.699068$

C $4.8265301 .017493-0.434030$

C $5.388047-0.296534-2.493229$

C $3.5739161 .436298-2.547994$

H 3.9991931 .4292080 .166749

H 5.3819960 .2928550 .178410

H $5.5173111 .829904-0.704018$

H $5.001718-0.751891-3.419846$

H $6.1527150 .441425-2.777619$

H 5.879227 -1.084730 -1.903042

H $4.2828762 .225434-2.837919$

H $3.1584070 .997569-3.470365$

H $2.7402451 .878045-1.975922$

H $2.786560-0.911463-2.228344$

$\mathrm{R}=i \mathrm{Pr}$

L1-Pd(Ar)-Cl( $\left.\mathrm{H}_{2} \mathrm{NR}\right)$

$E=-2469.00586500$

Pd -1.217452 $0.893117-0.598795$

P $2.212298-0.364809-0.232717$

P $-0.620803-0.8635700 .811058$

C $1.114703-1.2744450 .737050$

C $1.549315-2.6618871 .183661$

H $1.175173-3.4791390 .537729$

H $1.209069-2.8917602 .205817$

H $2.642698-2.7790161 .203135$

C $3.939128-0.7337490 .326022$

H $4.043994-1.8047990 .075081$

C $5.0388620 .006790-0.439102$

H $4.9895181 .083499-0.203186$

H $4.897258-0.082102-1.526932$

C $6.414304-0.531497-0.051169$

H $6.496305-1.579276-0.392957$

H $7.1993980 .029640-0.583117$

C $6.644085-0.4735261 .454800$

H 6.6884170 .5839991 .772660

H $7.621581-0.9137931 .709256$

C $5.525150-1.1737682 .218338$

H $5.681543-1.0761753 .304616$

H $5.549159-2.2566211 .997537$

C $4.159352-0.6118961 .837525$

H $3.351952-1.1158952 .386953$ 
H 4.1159210 .4467512 .132428

C $1.8945941 .452695-0.165450$

H $0.7803561 .513065-0.195880$

C $2.4218912 .299139-1.327202$

H $2.1327891 .875641-2.297813$

H $3.5253002 .311790-1.300235$

C $1.8698683 .719418-1.226699$

H $2.2792224 .332147-2.046151$

H $0.7796753 .657731-1.388664$

C 2.1700054 .3684210 .119084

H 3.2574434 .5487630 .208706

H 1.6873635 .3576590 .177432

C 1.7177533 .4905141 .280536

H 0.6168593 .4190481 .286922

H 2.0103533 .9399662 .243290

C 2.3014042 .0886691 .164171

H 3.4024942 .1549551 .206139

H 1.9850091 .4547782 .003220

C $2.258936-0.772189-2.053948$

H $3.027694-0.130492-2.520658$

C $2.648082-2.239319-2.263165$

H $3.656669-2.436409-1.866797$

H $1.954799-2.875331-1.685636$

C $2.596904-2.641261-3.734186$

H $2.849577-3.709128-3.835857$

H $3.374012-2.083684-4.287918$

C $1.236166-2.348903-4.352352$

H $1.231600-2.623662-5.419440$

H $0.470854-2.978560-3.863469$

C $0.867037-0.882511-4.173481$

H -0.136119 -0.680437 -4.582242

H $1.570507-0.254894-4.750513$

C $0.906662-0.472975-2.705529$

H $0.122480-1.017804-2.156932$

H $0.6472200 .593140-2.612137$

C -1.487289-2.496802 0.531354

H - $0.935944-3.2353721 .140418$

C -2.950693 -2.546656 0.978868

$\mathrm{H}-3.527571-1.7625920 .467207$

$\mathrm{H}-3.030503-2.3411402 .056947$

C -3.577282 -3.9057180 .678321$

H -3.082855 -4.683705 1.289393

H -4.636827 -3.897040 0.981332

C $-3.447738-4.272291-0.794678$

H -4.015166 -3.540958 -1.397923

H -3.897272 $-5.259681-0.989760$

C - $1.987240-4.253846-1.227427$

$\mathrm{H}-1.437564-5.046298-0.686903$

$\mathrm{H}-1.894975-4.488872-2.300991$

C - $1.341394-2.904945-0.934307$

H -0.274609 -2.925616 -1.206382

$\mathrm{H}-1.810266-2.128052-1.562800$

C -1.129320 -0.454642 2.554270

$\mathrm{H}-2.233049-0.4643032 .495037$

C - 0.7118250 .9618062 .937529

H 0.3895141 .0091072 .963801 
$\mathrm{H}-1.0359901 .6720162 .157992$

C -1.260386 1.3585164.303223

$\mathrm{H}-2.3638901 .3973374 .252336$

H -0.925667 2.375385 4.566588

C - 0.8396830 .3624885 .378405

$\mathrm{H}-1.2773710 .6357136 .352459$

H 0.2572930 .4131915 .505352

C - $1.230298-1.0616304 .999291$

$\mathrm{H}-0.876007-1.7739025 .762665$

$\mathrm{H}-2.332268-1.1446814 .984877$

C - $0.686263-1.4478873 .625712$

H - $1.003786-2.4716803 .370048$

H $0.417682-1.4557553 .658403$

C -3.149084 $0.555620-0.473408$

C -3.822155 -0.065359-1.534329

C -3.8874220.944927 0.651376

C $-5.190811-0.329598-1.452138$

H -3.274863 -0.362995 -2.432182

C -5.256022 0.6801260 .730594

H -3.394955 1.4484381 .487018

C $-5.9129040 .033258-0.315541$

H -5.695407 -0.824798 -2.286697

$\mathrm{H}-5.8118190 .9811141 .623136$

H -6.982793 -0.178050 -0.249646

$\mathrm{N}-1.4504172 .447104-1.823672$

C -2.319134 3.569397-1.519384

C -2.214421 4.630554-2.612529

C - $1.9771464 .159178-0.159025$

$\mathrm{H}-1.1808945 .007154-2.684757$

$\mathrm{H}-2.4898714 .212401-3.594296$

$\mathrm{H}-2.8831835 .483232-2.412780$

H -2.669621 4.971186 0.111839

$\mathrm{H}-2.0336913 .3829210 .619446$

$\mathrm{H}-0.9531284 .565420-0.158131$

$\mathrm{H}-1.6980382 .115619-2.758553$

H -3.383623 3.248927 -1.474199

TS (reductive elimination)

$E=-2468.98454899$

Pd - $1.257233-0.502411-0.865065$

P $2.230854-0.2511460 .419794$

P -0.4350881 .2302640 .421134$

C 1.2632131 .0741010 .944545

C 1.7369841 .8701402 .149403

H 2.8089051 .7324102 .355884

H 1.2185291 .6020813 .089732

H 1.5949422 .9546872 .015571

C 4.0161130 .1790650 .660118

H 4.1036620 .2485321 .759485

C $5.000395-0.9011010 .204440$

H $4.955974-0.998135-0.893928$

H 4.729907 -1.885310 0.616539

C $6.424555-0.5399380 .620629$

H $6.488701-0.5570521 .723787$

H $7.127054-1.3076380 .258004$

C 6.8321390 .8390550 .114430 
H $6.8876790 .816744-0.989158$

H 7.8438681 .0927910 .469671

C 5.8305601 .9075970 .538980

H 6.1149152 .8877890 .123740

H 5.8538032 .0179801 .638578

C 4.4153091 .5475450 .099108

H 3.6932022 .3150600 .410690

H $4.3795951 .521224-1.000114$

C $1.912333-0.710404-1.338993$

H $0.799514-0.609745-1.392486$

C $2.250018-2.137321-1.777398$

H $1.850135-2.881398-1.076008$

H $3.345342-2.274324-1.791878$

C $1.673835-2.398827-3.168058$

H $1.923222-3.423604-3.487423$

H $0.573170-2.347686-3.099305$

C $2.163439-1.384345-4.193800$

H $3.250785-1.512654-4.347984$

H $1.687867-1.570570-5.170248$

C $1.8899710 .041414-3.730982$

H $0.8000350 .214184-3.690912$

H $2.2997080 .769701-4.449416$

C $2.4771730 .290013-2.346991$

H $3.5738380 .171785-2.398065$

H $2.2793341 .317177-2.015176$

C $2.050051-1.8527991 .363115$

H $2.738375-2.5893280 .910496$

C $2.433777-1.6609792 .833870$

H $3.489119-1.3594302 .926731$

H $1.833367-0.8337043 .251445$

C $2.197572-2.9253633 .655225$

H $2.451820-2.7354704 .710653$

H $2.887759-3.7150943 .306627$

C $0.763918-3.4243903 .529339$

H $0.627674-4.3519914 .108387$

H $0.078348-2.6770653 .968240$

C $0.394421-3.6418512 .068041$

$\mathrm{H}-0.657213-3.9584501 .974759$

H $1.005059-4.4647921 .653295$

C $0.616916-2.3766721 .248932$

H - $0.069725-1.5913701 .597269$

H $0.338543-2.5539180 .198918$

C - 1.3969751 .5463951 .991727

$\mathrm{H}-0.8539722 .3347162 .544694$

C -2.8211892.046040 1.736137

$\mathrm{H}-3.3494871 .3322031 .084677$

$\mathrm{H}-2.8019113 .0056441 .198321$

C -3.601972 2.221152 3.036107

$\mathrm{H}-3.1513623 .0404093 .626834$

$\mathrm{H}-4.6336332 .5326712 .805313$

C -3.606609 0.949046 3.874049

$\mathrm{H}-4.1469400 .1592753 .323018$

$\mathrm{H}-4.1547041 .1088594 .817122$

C -2.184361 0.4801494 .153095

$\mathrm{H}-1.6688021 .2295314 .781612$

H -2.190095 -0.459116 4.731333 
C -1.4051320.287766 2.857597

$\mathrm{H}-0.370482-0.0207393 .074769$

$\mathrm{H}-1.861646-0.5309332 .274390$

C $-0.6933472 .841776-0.486084$

$\mathrm{H}-1.7929262 .878105-0.599436$

C - $0.1043772 .793823-1.891399$

H $0.9932682 .721516-1.807590$

H -0.446826 $1.879495-2.405006$

C $-0.4630014 .038911-2.695181$

$\mathrm{H}-1.5563534 .062504-2.854806$

$\mathrm{H}-0.0037693 .990896-3.696574$

C - $0.0316375 .310347-1.972231$

$\mathrm{H}-0.3388406 .203101-2.541337$

H $1.0723985 .338449-1.922203$

C $-0.5933075 .360623-0.555771$

$\mathrm{H}-0.2319066 .260122-0.030337$

$\mathrm{H}-1.6938975 .449951-0.603318$

C - 0.2313454 .1059270 .235671

H -0.660526 4.163414 1.248683

H 0.8650844 .0587610 .357601

C -3.267472 $-0.562079-0.810123$

C -3.911087-1.1376830.299350

C -3.973933 0.389433 -1.571864

C -5.210438 -0.7634500 .640044$

$\mathrm{H}-3.387665-1.8721430 .916234$

C -5.268842 $0.759517-1.218135$

$\mathrm{H}-3.4955990 .854551-2.438075$

C -5.900452 $0.189296-0.109601$

H -5.683879-1.218849 1.514723

H $-5.7908341 .510916-1.817481$

H -6.9155240 .4840160 .164426$

$\mathrm{N}-2.240156-1.888778-1.946101$

C -2.593257 -3.237922 -1.563602

C - $1.499662-4.232851-1.949981$

C -3.949918 -3.641277-2.129535

H -2.669903 -3.262719-0.461012

H - $0.538682-3.937349-1.503757$

$\mathrm{H}-1.744162-5.251136-1.605170$

$\mathrm{H}-1.366682-4.263402-3.043456$

$\mathrm{H}-4.731511-2.946075-1.788599$

$\mathrm{H}-3.924811-3.620903-3.232865$

H -4.231559-4.660595-1.823250

$\mathrm{H}-2.677634-1.612243-2.819465$

\section{L1-Pd-(ArNHR)}

$E=-2469.04340455$

Pd 1.3456940 .6244290 .678661

P - $2.187687-0.764986-0.019910$

P $0.0946830 .937422-1.152582$

C -1.479243 0.116390-1.311708

C -2.082254 0.004990-2.697799

$\mathrm{H}-2.1334820 .987517-3.202756$

H -3.112568 -0.384745 -2.692717

$\mathrm{H}-1.515174-0.659042-3.378703$ 
C -4.029198 -0.782132 -0.235639

H -4.174939-1.370404-1.160032

C -4.797303 -1.496789 0.878217

H -4.678785 -0.933957 1.819932

$\mathrm{H}-4.389211-2.5026631 .061257$

C -6.282379-1.592943 0.537157

$\mathrm{H}-6.405145-2.242853-0.348264$

$\mathrm{H}-6.825842-2.0848371 .360241$

C $-6.883631-0.2232070 .243638$

$\mathrm{H}-6.8679690 .3827821 .167814$

H -7.943018 -0.323052 -0.043145

C $-6.1001580 .506478-0.841902$

$\mathrm{H}-6.5169041 .513242-1.006502$

H -6.209725 -0.034737 -1.799507

C -4.619588 $0.607460-0.490598$

$\mathrm{H}-4.0584411 .120083-1.283834$

$\mathrm{H}-4.5037631 .2268020 .412624$

C -1.710126 -0.0988491 .618977$

$\mathrm{H}-0.6017920 .0210731 .449214$

C - $1.903289-0.9870092 .848437$

$\mathrm{H}-1.565269-2.0172312 .670790$

H -2.973757 -1.0517913.110665

C -1.134056 -0.3911214.026510

$\mathrm{H}-1.264665-1.0194954 .922794$

H -0.056251 -0.4071553.780653

C - 1.5587141 .0447504 .315653

$\mathrm{H}-2.6007891 .0411184 .685361$

$\mathrm{H}-0.9453371 .4682525 .128308$

C -1.471673 1.918329 3.069651

$\mathrm{H}-0.4169282 .0235432 .758949$

$\mathrm{H}-1.8470992 .9323913 .283358$

C -2.236820 1.3057801 .903291

$\mathrm{H}-3.3130681 .2621132 .150209$

$\mathrm{H}-2.1349831 .9305751 .006573$

C - $1.756158-2.5806820 .076037$

$\mathrm{H}-2.359481-3.0367230 .882313$

C -2.106244 -3.267887-1.247931

H -3.189549 -3.209781-1.440522

$\mathrm{H}-1.613691-2.718590-2.068520$

C -1.660560 -4.726097-1.280186

$\mathrm{H}-1.899309-5.166507-2.261969$

H -2.237944 $-5.300180-0.532565$

C $-0.174643-4.862490-0.976229$

H $0.124621-5.923248-0.982803$

H $0.408445-4.366209-1.772796$

C $0.160828-4.2183730 .361908$

H $1.242188-4.2863250 .559751$

H $-0.343206-4.7794391 .170779$

C - $0.271582-2.7572250 .407052$

H $0.325576-2.168368-0.305079$

H - $0.039118-2.3288371 .392864$

C $1.0722700 .323611-2.637030$

H $0.4522830 .519457-3.532508$

C $2.4045101 .052660-2.812003$

H $2.9768770 .951816-1.870945$

H 2.239764 2.129765 -2.966996 
C $3.2170490 .494036-3.977924$

H $2.6851040 .703226-4.924406$

H $4.1844441 .019706-4.045474$

C $3.435341-1.008427-3.850050$

H $4.054787-1.209713-2.958287$

H $3.994359-1.393941-4.718901$

C $2.106505-1.737273-3.696961$

H $1.519635-1.629306-4.627868$

H $2.273505-2.818786-3.557342$

C $1.306914-1.179800-2.525751$

H $0.340830-1.699231-2.436465$

H $1.854071-1.370279-1.583462$

C - $0.1759522 .729592-1.687259$

H -0.043492 2.749518-2.786499

C $0.8697223 .654367-1.057768$

H 0.7478393 .6061420 .039837

H $1.8877803 .284753-1.249300$

C $0.7198765 .093278-1.542619$

H $0.9367915 .135030-2.626032$

H $1.4674885 .739586-1.052963$

C - $0.6867015 .625293-1.292450$

H -0.795195 6.644377 -1.699377

H $-0.8508785 .706415-0.202211$

C -1.742273 4.696583-1.882560

$\mathrm{H}-2.7541825 .065110-1.644183$

$\mathrm{H}-1.6606924 .705012-2.985014$

C -1.571055 3.266113-1.380146

$\mathrm{H}-2.3438032 .606350-1.799710$

H -1.722604 3.254379-0.287016

C $3.623474-0.7946781 .339308$

C $3.331071-2.1647341 .395403$

C $4.621655-0.3531590 .459055$

C $3.989724-3.0636860 .563391$

H $2.556289-2.5178762 .081534$

C $5.281819-1.262071-0.361664$

H 4.8603990 .7086590 .391260

C $4.969116-2.620790-0.324936$

H $3.736950-4.1255740 .615510$

H $6.048706-0.896274-1.048737$

H $5.485906-3.326511-0.978102$

N 2.8948550 .1091592 .157551

C 3.6039801 .2385672 .785289

C 4.6969630 .7597153 .731137

C 2.5859532 .1320423 .469785

H 4.2624150 .1968804 .574142

H 5.4133400 .1037793 .214506

H 5.253202 1.612177 4.148457

H 3.0788373 .0013053 .928844

H 1.8420132 .4795622 .736077

H 2.0524841 .5872664 .267020

H $2.393480-0.4113762 .876609$

H 4.0577421 .8172071 .966676

2,6-diisopropylaniline 


$$
\text { L1-Pd(Ar)-Cl( } \left.\mathrm{H}_{2} \mathrm{NR}\right)
$$

$E=-2817.74414753$

Pd $-0.9705770 .103429-0.384747$

P $2.782301-0.613708-0.021587$

P 0.5357191 .3573900 .852121

C 2.1603020 .6501860 .961851

C 3.0659061 .0559782 .115822

H 2.8562910 .5128353 .057814

H 2.9841762 .1272772 .349838

H 4.1339270 .8870281 .896232

C $4.484434-0.176246-0.619850$

H $5.071102-0.2036610 .316612$

C $5.123327-1.189781-1.571070$

H $4.564632-1.207975-2.522182$

H $5.067181-2.209192-1.157680$

C $6.578273-0.821711-1.853148$

H $7.161452-0.925460-0.920091$

H $7.013356-1.535353-2.571214$

C $6.7091930 .606092-2.371428$

H $6.2202100 .678443-3.359973$

H $7.7696300 .858187-2.532172$

C $6.0599551 .605630-1.420059$

H $6.1306512 .627169-1.826939$

H $6.6142721 .613313-0.463855$

C $4.6009151 .252976-1.151408$

H $4.1459951 .951310-0.432808$

H $4.0272571 .355984-2.085630$

C $1.685818-1.033905-1.449876$

H $0.670444-0.940083-1.001125$

C $1.751602-2.470014-1.979544$

H $1.617928-3.196409-1.166201$

H $2.745832-2.659364-2.420898$

C $0.657905-2.692615-3.023233$

H $0.720274-3.724727-3.403097$

H - $0.322414-2.584996-2.526628$

C $0.736617-1.692014-4.168674$

H $1.669874-1.849777-4.740656$

H - $0.092807-1.859678-4.874355$

C $0.705422-0.262967-3.643884$

$\mathrm{H}-0.275007-0.054931-3.180837$

H $0.8259020 .460421-4.466200$

C $1.790496-0.031662-2.600492$

H $2.777761-0.147072-3.078912$

H $1.7358890 .992816-2.216201$

C $3.064454-2.2354860 .848351$

H $3.302390-2.9642540 .053211$

C $4.230146-2.2443691 .841992$

H $5.175270-1.9771831 .344500$

H $4.057098-1.4853332 .622414$

C $4.375811-3.6166442 .495316$

H $5.204592-3.5973023 .221355$

H $4.657615-4.3539271 .721730$

C $3.084495-4.0646533 .168589$

H $3.205776-5.0711893 .599867$

H $2.862771-3.3882014 .013697$ 
C $1.916373-4.0383292 .191135$

H $0.978940-4.3104972 .701077$

H $2.073485-4.8016261 .407315$

C $1.772216-2.6686501 .539164$

H $1.522599-1.9154062 .303209$

H $0.925660-2.6645680 .835782$

C - 0.0269811 .5347262 .623938

H 0.8592691 .8489833 .203942

C - 1.1207032 .5843332 .840821

$\mathrm{H}-1.9780502 .3677302 .185684$

H - 0.7575873 .5844602 .561333

C - 1.5931272 .6087954 .292170

$\mathrm{H}-0.7673682 .9525494 .942622$

H -2.4040393.346999 4.401926

C -2.055654 1.2347154 .759827

H -2.935508 0.9285884 .165755

$\mathrm{H}-2.3836101 .2737265 .811395$

C -0.948368 0.2041954 .580899

$\mathrm{H}-0.1020120 .4597365 .244884$

H -1.293766 -0.7970714 .887076$

C -0.4663230 .1621783 .136705$

H $0.361485-0.5541063 .027403$

$\mathrm{H}-1.277861-0.2100332 .487802$

C 0.6418923 .1268590 .279706

H -0.3897403 .4952310 .425484$

C $0.9510153 .216097-1.211880$

H $1.9720912 .831175-1.381605$

H $0.2685312 .561827-1.779001$

C $0.8665824 .652610-1.716537$

$\mathrm{H}-0.1764495 .005449-1.622357$

H $1.1136784 .694003-2.790189$

C $1.7850375 .574744-0.922547$

H $1.6763826 .616876-1.264442$

H $2.8355385 .291308-1.118398$

C 1.5147805 .4724160 .574217

H 2.2217486 .1042701 .136647

H 0.5051785 .8657610 .791088

C 1.5990314 .0272871 .059634

H 1.3964733 .9804072 .141394

H 2.6290553 .6575380 .913211

C -2.505980 $1.306055-0.075920$

C -3.5262690.904864 0.791756

C $-2.6261522 .526722-0.748355$

C -4.6301441 .7293891 .013208$

H -3.478975 -0.0652911 .292426$

C -3.738635 $3.344757-0.532248$

$\mathrm{H}-1.8559062 .852134-1.450692$

C - 4.7395432 .9546090 .355879

$\mathrm{H}-5.4149211 .3997551 .699081$

$\mathrm{H}-3.8201434 .293991-1.069163$

H -5.606674 3.5971800 .526177

$\mathrm{N}-2.154963-1.184034-1.433153$

$\mathrm{H}-2.335846-0.745138-2.331672$

C -3.351773 -1.648684 -0.919638

C $-4.601630-1.278667-1.495096$

C $-3.349100-2.4863340 .231290$ 
$\begin{array}{llll}\text { C }-5.783667 & -1.786570 & -0.957441 \\ \text { C }-4.558019 & -2.961836 & 0.737562 \\ \text { C }-5.775726 & -2.628424 & 0.150397 \\ \text { H }-6.739726 & -1.500442 & -1.401065 \\ \text { H }-4.551122 & -3.612705 & 1.614883 \\ \text { H }-6.712299 & -3.011592 & 0.561947 \\ \text { C }-4.623993 & -0.320058 & -2.668630 \\ \text { C }-5.897978 & 0.511013 & -2.765719 \\ \text { C }-4.346197 & -1.049985 & -3.987060 \\ \text { H }-3.804964 & 0.401704 & -2.494413 \\ \text { H }-6.105955 & 1.024878 & -1.815513 \\ \text { H }-5.792320 & 1.276350 & -3.550378 \\ \text { H }-6.774739 & -0.102650 & -3.027921 \\ \text { H }-3.414301 & -1.635619 & -3.943351 \\ \text { H }-5.161324 & -1.756711 & -4.210250 \\ \text { H }-4.268266 & -0.342428 & -4.828761 \\ \text { C }-2.032888 & -2.902446 & 0.855196 \\ \text { C }-1.453797 & -4.093449 & 0.090186 \\ \text { C }-2.112507 & -3.185641 & 2.350459 \\ \text { H }-1.339331 & -2.050545 & 0.727126 \\ \text { H }-1.339528 & -3.844986 & -0.974365 \\ \text { H }-0.471918 & -4.398099 & 0.487898 \\ \text { H }-2.129691 & -4.960244 & 0.165543 \\ \text { H }-2.592085 & -2.356960 & 2.893596 \\ \text { H }-2.680336 & -4.103910 & 2.569750 \\ \text { H }-1.103151 & -3.321946 & 2.768907\end{array}$

TS (reductive elimination)

$E=-2817.71870665$

Pd $0.9372570 .054649-0.077409$

P -2.905383 $0.249398-0.650088$

P $-0.732077-0.2215621 .477758$

C -2.427390 -0.0857210 .969851$

C -3.506098 -0.0885042 .039881$

$\mathrm{H}-3.688265-1.0872312 .478142$

$\mathrm{H}-4.4815500 .2642591 .671012$

$\mathrm{H}-3.2567900 .5797352 .883236$

C -4.599907 -0.441410 -0.934804

H $-5.2376950 .166721-0.268192$

C -5.130034 -0.253777 -2.358197

$\mathrm{H}-4.518512-0.850171-3.056552$

H -5.040178 $0.795054-2.680509$

C -6.584872 $-0.705587-2.457477$

H -7.210848 $-0.037805-1.838187$

H -6.942539 -0.592626 -3.493644

C -6.761636 -2.143809-1.984503

$\mathrm{H}-6.229746-2.819903-2.678375$

$\mathrm{H}-7.824257-2.432441-2.025266$

C -6.207245 -2.338574 -0.577696

H $-6.300400-3.392342-0.269610$

H $-6.810308-1.7514830 .138757$

C $-4.749848-1.897859-0.484763$

$\mathrm{H}-4.365700-2.0229030 .536706$

H -4.135232 -2.547846 -1.126178 
C -1.727162 -0.382433-1.911953

$\mathrm{H}-0.752340-0.107818-1.436258$

C - $1.7474810 .254796-3.303971$

$\mathrm{H}-1.7886951 .350868-3.255126$

$\mathrm{H}-2.648418-0.067806-3.853733$

C - $0.498223-0.170422-4.074678$

$\mathrm{H}-0.5055250 .281231-5.079678$

H $0.3892470 .231857-3.553134$

C - $0.379618-1.687081-4.174908$

H -1.197574 -2.073838-4.810055

H $0.560808-1.963328-4.679342$

C $-0.459794-2.344290-2.802467$

H $0.422153-2.054020-2.204710$

H -0.436532 -3.442189-2.894431

C -1.705192 -1.905401-2.040684

$\mathrm{H}-2.603480-2.242649-2.587567$

$\mathrm{H}-1.734999-2.372460-1.048406$

C -3.0977712.056163-1.070196

H -3.474158 2.123712 -2.106838

C $-4.1122892 .719619-0.133829$

$\mathrm{H}-5.1057452 .256840-0.245669$

H -3.796458 2.5400790 .908731

C -4.222191 4.221555-0.375556

H -4.935790 4.6620220.339522

$\mathrm{H}-4.6420074 .397435-1.382614$

C -2.865665 4.905311 -0.272678

$\mathrm{H}-2.9606425 .983694-0.478101$

$\mathrm{H}-2.4896614 .8154550 .762602$

C $-1.8657114 .264947-1.225516$

$\mathrm{H}-0.8765844 .738883-1.123605$

H -2.187890 4.444647 -2.267549

C -1.742893 $2.763800-0.985664$

$\mathrm{H}-1.3191452 .5826630 .013004$

H -1.022650 2.331604 -1.695529

C -0.4997250 .9482222 .916275$

$\mathrm{H}-1.2095060 .6409123 .705411$

C 0.9188120 .8565983 .480058

H 1.6270341 .1259912 .677591

H $1.170366-0.1768163 .762664$

C 1.1106311 .7903064 .670980

H 0.4642561 .4558385 .503129

H 2.1477991 .7230365 .038464

C 0.7662393 .2315214 .313471

H 1.4956783 .6000373 .570490

H 0.8646623 .8843395 .196137

C - 0.6370873 .3351373 .726537

$\mathrm{H}-1.3784513 .0828144 .506543$

H -0.851520 4.372677 3.419737

C -0.8200932.390998 2.542748

$\mathrm{H}-1.8460772 .4586262 .149360$

H -0.1473362.699202 1.721324

C - $0.467083-1.8760702 .320560$

H $0.633359-1.9288212 .419659$

C -0.887738 -3.004527 1.384651

$\mathrm{H}-1.973628-2.9233701 .194661$

H $-0.386925-2.8709370 .413998$ 
C - $0.557250-4.3779271 .957385$

H $0.540500-4.4809932 .015514$

H -0.912217 -5.168075 1.274697

C $-1.152253-4.5653713 .347390$

$\mathrm{H}-0.863496-5.5447703 .762944$

H -2.255554 -4.570377 3.274997

C - $0.719358-3.4406834 .279873$

$\mathrm{H}-1.174597-3.5667765 .276158$

H $0.374792-3.4924684 .426352$

C - $1.077965-2.0702813 .709955$

$\mathrm{H}-0.736841-1.2860994 .402545$

H -2.175426 -1.979677 3.651975

C $2.521046-1.1496510 .366494$

C $3.409034-0.7298651 .370860$

C $2.371363-2.5299280 .132904$

C $4.060604-1.6669602 .168434$

H 3.5927220 .3351531 .525533

C $3.040090-3.4563560 .930292$

H $1.717078-2.883040-0.667689$

C $3.880195-3.0360681 .962774$

H $4.728616-1.3157892 .959748$

H $2.899162-4.5233490 .736500$

H 4.399751 -3.765209 2.587693

N $2.615920-0.090184-1.249110$

C $3.8361920 .578531-1.289381$

C $4.965191-0.072839-1.856882$

C $3.9741951 .880934-0.745864$

C $6.1833790 .602347-1.911776$

C $5.2225932 .504393-0.806928$

C $6.3223211 .883560-1.387883$

H $7.0507440 .109371-2.354805$

H $5.3361703 .509234-0.395587$

H $7.2878822 .393118-1.424485$

C $4.822749-1.475626-2.419092$

C $6.117616-2.279289-2.414403$

C $4.212959-1.443322-3.825733$

H $4.130248-2.017004-1.751672$

H $6.577142-2.287456-1.415012$

H $5.916165-3.321725-2.705285$

H $6.855485-1.880818-3.128769$

H $3.258897-0.892554-3.854695$

H $4.895830-0.935841-4.525254$

H $4.030330-2.461798-4.204726$

C $2.7781512 .632457-0.197780$

C $2.0013693 .275173-1.347183$

C 3.1125183 .6543180 .880372

H 2.0996061 .8891850 .271953

H $1.6970902 .515349-2.082521$

H $1.0953403 .778906-0.976516$

H $2.6233134 .022891-1.864770$

H 3.6912813 .2042841 .701886

H 3.6909274 .5054790 .486918

H 2.1847184 .0677221 .305483

H $2.613320-0.874736-1.887395$ 


$$
\text { L1-Pd-(ArNHR) }
$$

$E=-2817.75787699$

Pd $-0.886896-0.0256490 .240382$

P $2.9333170 .028815-0.723615$

P $0.905607-0.2628631 .566397$

C $2.574039-0.2108670 .937384$

C $3.691274-0.6027981 .884153$

H $3.646540-0.0316482 .830265$

H $4.695444-0.4208991 .470445$

H 3.678331 -1.672209 2.170639

C $4.6265710 .768145-0.877856$

H $5.288237-0.031542-0.497895$

C $5.0658511 .069148-2.312574$

H $4.4303501 .870286-2.727340$

H $4.9286020 .192043-2.963383$

C $6.5243261 .519161-2.349326$

H $7.1666430 .677984-2.031113$

H $6.8175131 .758377-3.384473$

C $6.7697442 .713877-1.434991$

H $6.2183763 .587664-1.827553$

H $7.8362692 .991176-1.444546$

C $6.3018132 .429753-0.012024$

H 6.4402503 .3191660 .623636

H 6.9294391 .6333600 .428108

C 4.8415961 .9908440 .018824

H 4.5165621 .7731871 .045329

H $4.2077842 .820002-0.331855$

C $1.6734491 .056720-1.571188$

H $0.7378490 .582365-1.162766$

C $1.6113951 .001165-3.098403$

H $1.655818-0.029431-3.475157$

H $2.4756001 .530967-3.535410$

C $0.3210151 .660700-3.580028$

H $0.2676041 .633287-4.680554$

H -0.529825 $1.059138-3.210773$

C $0.1910013 .094178-3.076809$

H $0.9727873 .713202-3.553811$

H -0.774103 3.525113 -3.390524

C $0.3488853 .172808-1.563224$

$\mathrm{H}-0.4851972 .643474-1.066523$

H $0.3111114 .220477-1.222524$

C $1.6399762 .509483-1.101317$

H $2.5017613 .060116-1.519160$

H $1.7260502 .552168-0.007923$

C $3.044025-1.511115-1.774730$

H $3.354321-1.214442-2.793069$

C $4.096808-2.463604-1.199084$

H $5.096412-2.001494-1.233543$

H $3.870118-2.636302-0.132713$

C $4.130138-3.801714-1.930453$

H $4.871977-4.465724-1.457518$

H 4.473986 -3.640204 -2.968458

C $2.757212-4.459937-1.956789$

H $2.797775-5.414140-2.506753$

H $2.450701-4.705745-0.923936$ 
C $1.725972-3.526741-2.576585$

H $0.727226-3.991672-2.566442$

H $1.981048-3.360264-3.639560$

C $1.672486-2.187433-1.850320$

H $1.296342-2.331823-0.827210$

H $0.936366-1.528269-2.333123$

C $0.873737-1.8597922 .563952$

H $1.702816-1.7844193 .292456$

C -0.422987 -2.058643 3.348324

$\mathrm{H}-1.272683-2.0025182 .643081$

H - $0.566480-1.2397694 .067695$

C - $0.447257-3.3886534 .098751$

H $0.320412-3.3676964 .893906$

$\mathrm{H}-1.416802-3.5119804 .609581$

C $-0.173426-4.5732863 .181140$

H $-0.999404-4.6680802 .452717$

H $-0.157790-5.5137503 .756117$

C $1.134352-4.3800192 .424699$

H $1.975324-4.3907673 .142111$

H $1.308915-5.2190171 .729819$

C $1.138746-3.0603981 .663015$

H $2.093026-2.9226761 .133695$

H $0.354555-3.0850000 .885649$

C 0.8477360 .9736342 .995876

H 1.2701170 .4569443 .879847

C -0.5907081 .3949043 .308945$

$\mathrm{H}-1.0021611 .8809162 .408247$

$\mathrm{H}-1.2400280 .5253383 .479753$

C - 0.6648462 .3526264 .492934

$\mathrm{H}-0.3335331 .8314545 .410316$

$\mathrm{H}-1.7125252 .6503084 .664385$

C 0.2085503 .5812544 .269570

H 0.1910564 .2403845 .153525

H - 0.2114464 .1697943 .432898

C 1.6397373 .1842903 .924163

H 2.246605 4.080186 3.709740

H 2.1047352 .6973804 .801015

C 1.6824862 .2248672 .738814

H 2.7181581 .9576662 .483059

H 1.2712972 .7393751 .852530

C -3.362900 1.1921230.496289

C -4.197729 0.5236531 .396773

C -3.026589 2.531526 0.740515

C -4.646298 1.1741092 .543428

H -4.484766 -0.5089771 .199248$

C -3.482923 3.1727661 .886140

$\mathrm{H}-2.3800913 .0612330 .036173$

C -4.2907132.4969172.801409

H -5.286804 0.6336123 .244568

H -3.1990514.2124212.066144

H -4.6451963.0001043.703157

$\mathrm{N}-2.8684750 .539305-0.674129$

C -3.795329 -0.308078-1.389099

C -3.801367 -1.703207-1.209329

C -4.714693 0.320344-2.261739

C -4.738355 -2.452631-1.934391 

C -5.617552 -0.472461 -2.969785
C -5.633838 -1.854076-2.806934
$\mathrm{H}-4.755768-3.537817-1.808972$
$\mathrm{H}-6.328201-0.003336-3.652130$
$\mathrm{H}-6.351517-2.463272-3.361600$
C -4.690978 $1.825619-2.463099$
C -6.053844 2.421935-2.798143
C -3.658197 2.206523 -3.528057
$\mathrm{H}-4.3850942 .282579-1.509026$
H -6.814817 2.119557-2.063699
H -5.994290 3.520836 -2.794007
$\mathrm{H}-6.4042782 .121906-3.798118$
$\mathrm{H}-2.6543021 .815509-3.300396$
$\mathrm{H}-3.9449091 .791490-4.507044$
H -3.577962 $3.300255-3.629637$
C - $2.837624-2.448532-0.310234$
C - $1.879355-3.294041-1.146263$
C -3.552103 -3.291979 0.742253
$\mathrm{H}-2.210420-1.7023910 .228288$
$\mathrm{H}-1.323496-2.663008-1.854692$
$\mathrm{H}-1.145738-3.797865-0.498669$
$\mathrm{H}-2.413283-4.069167-1.719852$
H -4.234896 -2.686692 1.357368
$\mathrm{H}-4.142836-4.1058530 .291372$
$\mathrm{H}-2.817138-3.7540751 .418824$
H -2.492542 $1.238190-1.310098$

\subsubsection{Catalytic cycle for $p$-F-phenylchloride}

Table S20. SCF energies and enthalpy/free energy corrections for the catalytic cycle with $p$ fluorochlorobenzene and $\mathrm{N}$-methyaniline.

\begin{tabular}{ccccc}
\hline & Corr(G) & $\mathbf{E}(\mathbf{P C M})$ & $\Delta \mathbf{G}(\mathbf{P C M}) / \mathbf{E}_{\mathbf{H}}$ & $\boldsymbol{\Delta G}(\mathbf{P C M}) / \mathbf{k J} / \mathbf{m o l}$ \\
ArCl & 0.052648 & -790.718616 & & \\
HNPhMe & 0.114619 & -326.653575 & & \\
ArNPhMe & 0.179741 & -656.706817 & & \\
& & & & \\
Energies with L1 & & & & 151.68 \\
\hline L1-Pd(Ar)-NMe 2 & 0.987851 & -2719.360698 & 0.057772 & 225.80 \\
TS (RE) & 0.987292 & -2719.331907 & 0.086004 & 129.18 \\
L1-Pd-ArNMe 2 & 0.987945 & -2719.369362 & 0.049202 & 110.88 \\
L1-Pd-ArCl & 0.857987 & -2853.385267 & 0.042231 & 162.54 \\
TS (OA) & 0.859197 & -2853.366800 & 0.061908 & 29.44 \\
L1-Pd(Ar)-Cl & 0.862592 & -2853.420890 & 0.011214 & 0.00 \\
1/2 [L1-Pd(Ar)-Cl] 2 & 0.8763615 & -2853.445873 & 0.000000 & 51.54 \\
TS cis-trans-isomerization & 0.864559 & -2853.414441 & 0.019630 & 45.45 \\
L1-Pd(Ar)-Cl-o & 0.863149 & -2853.415350 & 0.017311 & 23.02 \\
L1-Pd(Ar)-Cl-HNMe 2 & 1.003524 & -3180.103224 & 0.008768 & \\
& & & & \\
\hline
\end{tabular}




\begin{tabular}{ccccc}
\hline Energies with L2 & & & & \\
\hline \hline L2-Pd(Ar)-NMe 2 & 0.646204 & -2057.245547 & 0.061612 & 161.76 \\
TS (RE) & 0.644585 & -2057.217099 & 0.088442 & 232.20 \\
L2-Pd-ArNMe 2 & 0.644735 & -2057.255299 & 0.050391 & 132.30 \\
L2-Pd-ArCl & 0.514804 & -2191.270932 & 0.043720 & 114.79 \\
TS (OA) & 0.513427 & -2191.251575 & 0.061699 & 161.99 \\
L2-Pd(Ar)-Cl & 0.518632 & -2191.307806 & 0.010674 & 28.02 \\
1/2 [L2-Pd(Ar)-Cl] 2 & 0.531721 & -2191.331569 & 0.000000 & 0.00 \\
Rotation & 0.519451 & -2191.287944 & 0.031355 & 82.32 \\
L2-Pd(Ar)-Cl (distal) & 0.516835 & -2191.298256 & 0.018427 & 48.38 \\
TS cis-trans-isomerization & 0.518345 & -2191.295381 & 0.022812 & 59.89 \\
L2-Pd(Ar)-Cl-o & 0.518412 & -2191.298299 & 0.019961 & 52.41 \\
L2-Pd(Ar)-Cl-HNMe 2 (distal) & 0.65751 & -2517.985853 & 0.010461 & 27.46 \\
Energies with L3 & & & & \\
\hline L3-Pd(Ar)-NMe 2 & 0.525255 & -1597.857593 & 0.048661 & 127.76 \\
TS (RE) & 0.526761 & -1597.834430 & 0.073330 & 192.53 \\
L3-Pd-ArNMe 2 & 0.526074 & -1597.875377 & 0.031696 & 83.22 \\
L3-Pd-ArCl & 0.395587 & -1731.891042 & 0.024437 & 64.16 \\
TS (OA) & 0.398124 & -1731.873346 & 0.044669 & 117.28 \\
L3-Pd(Ar)-Cl & 0.399439 & -1731.918951 & 0.000380 & 1.00 \\
1/2 [L3-Pd(Ar)-Cl] 2 & 0.414560 & -1731.934451 & 0.000000 & 0.00 \\
TS cis-trans-isomerization & 0.400446 & -1731.911126 & 0.009212 & 24.18 \\
L3-Pd(Ar)-Cl - o & 0.400129 & -1731.911946 & 0.008074 & 21.20 \\
L3-Pd(Ar)-Cl-HNMe 2 & 0.540873 & -2058.592921 & 0.006800 & 17.85 \\
\hline
\end{tabular}

\subsubsection{Energies and coordinates related to Table 4}

Table S21. SCF energies and enthalpy/free energy corrections for the formation of the complex $\mathrm{LPd}(\mathrm{ArCl})$. Compounds related to Table 4.

\begin{tabular}{|c|c|c|c|c|c|c|c|c|}
\hline & $\begin{array}{l}\text { Corrpbeo( } \\
\text { G) }\end{array}$ & $\begin{array}{c}\mathrm{E}(\mathrm{PCM})_{\mathrm{PB}} \\
\mathrm{E}^{-}\end{array}$ & $\begin{array}{l}\text { Corrm06 } \\
\text { (G) }\end{array}$ & $\begin{array}{c}\mathrm{E}(\mathrm{PCM})_{\mathrm{M0}} \\
6\end{array}$ & $\underset{B E_{0} / E_{H}}{\Delta G(P C M) P}$ & $\begin{array}{c}\Delta \mathrm{G}(\mathrm{PCM}) \\
\mathrm{PBEO} / \\
\mathrm{kJ} / \mathrm{mol}\end{array}$ & $\begin{array}{c}\Delta \mathbf{G}(\mathrm{PCM})_{\mathrm{M} 06} \\
/ \mathrm{E}_{\mathrm{H}}\end{array}$ & $\begin{array}{c}\Delta G(P C \\
M)_{\text {M06/ }} \\
\mathrm{kJ} / \mathrm{mol}\end{array}$ \\
\hline $\mathrm{dba}$ & 0.214722 & -730.86848 & 0.210495 & -731.14959 & & & & \\
\hline $\mathrm{PhCl}$ & 0.062051 & -691.52644 & 0.060739 & -691.73795 & & & & \\
\hline
\end{tabular}

\section{Energies with L1}

\begin{tabular}{ccccccccc}
\hline \hline $\mathrm{L}_{2} \cdot \mathrm{Pd}$ & 1.593734 & & & & & & & \\
& & -3998.5760 & 1.583803 & -3999.8870 & -0.043877 & -115.20 & -0.031196 & -81.91 \\
$\mathrm{~L} \cdot \mathrm{Pd}(\mathrm{dba})$ & 1.026035 & & & & & & & \\
& & -2793.5702 & 1.013642 & -2794.5129 & -0.029489 & -77.42 & -0.018796 & -49.35 \\
$\mathrm{~L} \cdot \mathrm{Pd}$ & 0.784348 & & & & & & & \\
$\mathrm{~L} \cdot \mathrm{Pd}(\mathrm{PhCl})$ & 0.867313 & -2754.1927 & 0.855031 & -2755.0736 & 0.000000 & 0.00 & 0.000000 & 0.00 \\
\hline
\end{tabular}




\begin{tabular}{|c|c|c|c|c|c|c|c|c|}
\hline L1 & 0.786524 & -1935.8639 & 0.775236 & -1936.5059 & & & & \\
\hline \multicolumn{9}{|c|}{ Energies with L2 } \\
\hline $\mathrm{L}_{2} \cdot \mathrm{Pd}$ & 0.911418 & -2674.3502 & 0.901069 & -2675.2261 & -0.037332 & -98.01 & -0.030142 & -79.14 \\
\hline$L \cdot P d(d b a)$ & 0.682803 & -2131.4622 & 0.675019 & -2132.1889 & -0.035472 & -93.13 & -0.025740 & -67.58 \\
\hline$L \cdot P d$ & 0.441412 & -1400.5248 & 0.435041 & -1400.9815 & 0.006766 & 17.76 & 0.002627 & 6.90 \\
\hline$L \cdot P d(P h C l)$ & 0.524165 & -2092.0787 & 0.518064 & -2092.7443 & 0.000000 & 0.00 & 0.000000 & 0.00 \\
\hline L2 & 0.442157 & -1273.7534 & 0.43659 & -1274.1825 & & & & \\
\hline \multicolumn{9}{|c|}{ Energies with L3 } \\
\hline $\mathrm{L}_{2} \cdot \mathrm{Pd}$ & 0.672769 & -1755.5890 & 0.666172 & -1756.1871 & -0.036302 & -95.31 & -0.032987 & -86.61 \\
\hline$L \cdot P d(d b a)$ & 0.562343 & -1672.0676 & 0.55534 & -1672.6572 & -0.022540 & -59.18 & -0.015864 & -41.65 \\
\hline$L \cdot P d$ & 0.325397 & -941.13982 & 0.320694 & -941.45866 & 0.014545 & 38.19 & 0.008936 & 23.46 \\
\hline$L \cdot P d(P h C l)$ & 0.405493 & -1632.6988 & 0.401252 & -1633.2254 & 0.000000 & 0.00 & 0.000000 & 0.00 \\
\hline L3 & 0.328031 & -814.37896 & 0.323539 & -814.66460 & & & & \\
\hline
\end{tabular}

Structures (PBE0)

CyJohnPhos

$\mathrm{L}_{2} \cdot \mathrm{Pd}$

$E=-2674.35019129$

Pd $0.183567-0.050409-0.347525$

P 2.3166490 .2722680 .405978

P -2.049416 0.458471 -0.202809

C $2.917213-0.7328811 .869467$

H $3.696792-0.1432092 .389828$

C $1.745076-0.9812862 .825572$

H $1.318084-0.0295323 .172507$

H $0.939642-1.4753462 .253990$

C $2.140935-1.8457754 .017162$

H $2.875676-1.3033244 .640255$

H $1.259967-2.0204034 .656268$

C $2.744413-3.1693183 .565751$

H $3.063330-3.7689374 .433915$

H $1.967960-3.7586843 .046638$

C $3.914261-2.9387732 .617768$

H $4.318110-3.8998152 .259078$

H $4.735191-2.4458673 .170089$

C $3.526981-2.0661471 .427919$

H $2.790905-2.5978980 .798234$

H $4.405409-1.8962240 .789146$

C 2.6613572 .0395060 .897140

H 3.7341402 .1278251 .148410

C $2.3601842 .944424-0.299512$

H $1.3138452 .758566-0.605403$

H $2.9911902 .658166-1.156818$

C 2.5506764 .4208750 .028585

H 3.6199624 .6165230 .228356

H $2.2825925 .036569-0.846008$ 
C 1.7285364 .8328011 .243576 H 1.9080015 .8916601 .491366 H 0.6551664 .7486830 .999344 C 2.0394703 .9449212 .441745 H 1.4088764 .2227783 .302218 H 3.0851844 .1165912 .756410 C 1.8502482 .4648142 .120785 H 0.7846042 .2473551 .926924 H 2.1371561 .8624232 .996322 C $3.6866120 .006022-0.810610$ C $4.9979680 .371941-0.471769$ H 5.1872450 .8592130 .488148 C $6.0751590 .108351-1.310099$ H $7.0845250 .405804-1.015758$ C $5.854162-0.546367-2.520435$ H $6.687588-0.763363-3.192920$ C $4.562830-0.918015-2.873757$ H $4.381879-1.416895-3.828856$ C $3.462328-0.647210-2.042247$ C $2.127330-1.080007-2.532423$ C $1.087086-0.151564-2.746059$ H $1.2770580 .912193-2.584254$ C $-0.119162-0.568174-3.325517$ $\mathrm{H}-0.9034510 .164927-3.516884$ C $-0.314706-1.905132-3.670284$ $\mathrm{H}-1.266664-2.221714-4.102089$ C $0.704220-2.830306-3.445885$ H $0.556764-3.881633-3.703546$ C $1.913659-2.418834-2.883762$ H $2.709113-3.147198-2.706417$ C -2.444552 2.211581-0.698747 H -3.541022 2.326431-0.779181 C - 1.9383703 .1953100 .359554 $\mathrm{H}-2.4468893 .0239581 .318610$ $\mathrm{H}-0.8673172 .9930550 .534449$ C -2.130115 4.648366 - 0.063104 $\mathrm{H}-3.2113124 .868950-0.125326$ $\mathrm{H}-1.7240165 .3169670 .713961$ C -1.481908 4.938643-1.410105 $\mathrm{H}-0.3865234 .824946-1.318071$ $\mathrm{H}-1.6627925 .983604-1.709911$ C -1.994462 3.976331-2.473091 $\mathrm{H}-1.4895234 .156915-3.436080$ $\mathrm{H}-3.0701834 .162900-2.646717$ C -1.791961 2.526389-2.049339 $\mathrm{H}-0.7120622 .313608-1.950724$ $\mathrm{H}-2.1661061 .848931-2.831627$ C -2.859126 0.1880081 .453176 H -2.939587 -0.9120531 .466400$ C - 1.9622300 .5479092 .637417 $\mathrm{H}-0.9680860 .1017652 .481682$ $\mathrm{H}-1.8049211 .6385182 .687423$ 
C -2.5907870.070147 3.942028

H -2.628514 -1.034490 3.936976

H - 1.9565350 .3561354 .797310

C - 4.0020440 .6231704 .116554

H -3.947531 1.722545 4.221061

H -4.452663 0.2464335 .049413

C -4.8898320 .2803232 .924141$

H -5.892828 0.7201363 .050966

H -5.032996 -0.814775 2.882797

C -4.2715290 .7457361 .607067$

H -4.245874 1.8498171 .590655

H -4.899876 0.4325660 .757098

C - $3.175020-0.557206-1.266156$

C -4.039427 $0.016100-2.208910$

$\mathrm{H}-4.1125871 .101133-2.289955$

C - $4.829962-0.762956-3.051567$

H -5.487894 -0.278904-3.777498

C $-4.784928-2.149737-2.955652$

H -5.399802 -2.773069-3.609053

C - $3.951276-2.738827-2.008467$

H -3.912084 -3.826984 -1.913983

C $-3.139372-1.969188-1.167139$

C - $2.306219-2.695902-0.167434$

C - $0.914725-2.791960-0.299377$

H - $0.420726-2.340654-1.162249$

C $-0.169060-3.5197990 .627631$

H $0.910784-3.6033360 .491859$

C $-0.793490-4.1353431 .710376$

H -0.203517-4.696870 2.439019

C - $2.177737-4.0439421 .851599$

H -2.678506 -4.530652 2.692036

C - $2.927540-3.3383260 .911818$

H -4.013913 -3.271210 1.016496

$L \cdot P d(d b a)$

$E=-2131.46219314$

Pd $-0.038006-0.8415580 .691827$

P $0.9079701 .003342-0.316230$

O - $2.483334-3.2580932 .139248$

C - $0.0342841 .690674-1.753190$

H $0.4877782 .586422-2.134193$

C - $0.1077340 .668183-2.887617$

H - $0.559959-0.257578-2.491075$

H $0.9013200 .395000-3.234194$

C $-0.9403301 .192960-4.052772$

H -0.430076 2.066195 -4.498243

H -0.997715 $0.430733-4.846771$

C -2.338050 $1.600335-3.602406$

H -2.881836 $0.698672-3.269193$

H -2.911641 $2.011076-4.449049$

C -2.286989 2.603969-2.455964

H - $1.8641013 .555888-2.825301$ 
H -3.304470 2.831071 -2.099306

C -1.439014 2.093049-1.296089

H - $1.3778912 .854822-0.502082$

$\mathrm{H}-1.9225131 .209575-0.839575$

C $2.6024970 .606772-0.958808$

H $2.416823-0.284178-1.588192$

C 3.4917890 .1626360 .205226

H 3.6237951 .0109010 .901458

H $2.984981-0.6353790 .772775$

C $4.854988-0.308903-0.287190$

H $4.717889-1.229162-0.880309$

H $5.489595-0.5816890 .571834$

C $5.5407030 .748872-1.144083$

H $6.5038690 .371346-1.524226$

H $5.7762751 .630059-0.519639$

C $4.6479311 .183341-2.300820$

H $5.1395091 .970919-2.895048$

H $4.4922280 .326967-2.981524$

C $3.2907921 .675556-1.802491$

H $2.6594531 .967356-2.657673$

H $3.4388742 .582777-1.191070$

C - $2.083810-2.7663001 .088882$

C $-0.688309-2.9128890 .658907$

H - $0.096649-3.5496991 .323392$

C - $0.173926-2.515977-0.602093$

H - $0.883275-2.263513-1.397193$

C $1.152574-2.911143-1.112751$

C $1.428305-2.788410-2.483951$

H $0.642227-2.427537-3.152503$

C $2.678204-3.118743-3.002734$

H $2.864945-3.013523-4.074415$

C $3.683364-3.591001-2.160934$

H $4.663929-3.854322-2.563980$

C $3.422662-3.724675-0.795073$

H $4.203647-4.091161-0.124271$

C $2.178559-3.385311-0.276653$

H 1.999412 -3.473387 0.797089

C -2.973294 -1.8911950.273740

H -2.616163 -1.537839 -0.695454

C $-4.161072-1.4888960 .759812$

H -4.430437 -1.875641 1.749502

C -5.101362 -0.5684360 .127251$

C $-4.915850-0.066087-1.172807$

H $-4.053675-0.392193-1.757390$

C $-5.8166480 .833190-1.728241$

H -5.650943 1.208441 -2.741356

C -6.931936 1.252967-0.998911

H -7.640327 $1.960277-1.436483$

C -7.137260 0.7584880 .287702

H -8.008884 1.0772080 .864330

C -6.232565 - 0.1431370 .842343

H -6.394301-0.528149 1.852629 
C 1.1890712 .4772990 .759625

C 1.4349343 .7412640 .204979

C 1.2416032 .3347382 .165500

C 1.7452684 .8393381 .002220

H $1.3899953 .875250-0.877437$

C 1.5772033 .4428932 .954814

C 1.8280694 .6863612 .383935

H 1.9289665 .8125670 .541115

H 1.6172253 .3186044 .039624

H 2.0770465 .5387793 .020328

C 0.9461801 .0460152 .840706

C -0.3468380 .4795932 .736043$

C 1.9199030 .3775413 .578410

C -0.618709 -0.7678503 .317698$

$\mathrm{H}-1.1520951 .0611492 .278331$

C $1.635888-0.8555234 .180634$

H 2.9199030 .8104053 .659372

C $0.383217-1.4356264 .039070$

H -1.619313 -1.201197 3.253585

H $2.414930-1.3683664 .749963$

H $0.162714-2.4072444 .485734$

$L \cdot P d$

$E=-1400.52480639$

Pd $-0.650446-0.703067-1.619957$

P 0.5985480 .2344450 .022162

C 0.6768422 .0838470 .260703

H 1.1487042 .2753281 .242678

C $1.4914132 .787517-0.824429$

H $1.1093982 .474004-1.813428$

H $2.5440242 .469888-0.779165$

C $1.4180454 .307386-0.700571$

H 1.9171324 .6177800 .235468

H $1.9846604 .774480-1.522568$

C $-0.0203294 .807823-0.687407$

H $-0.4909144 .587878-1.662793$

$\mathrm{H}-0.0459745 .903220-0.568521$

C -0.8188554 .1284350 .417626$

$\mathrm{H}-0.4118644 .4303251 .399862$

$\mathrm{H}-1.8684184 .4642400 .400811$

C - 0.7619502 .6104080 .293291

$\mathrm{H}-1.3166732 .1387201 .119481$

H -1.268895 $2.292783-0.636974$

C $2.337395-0.4033190 .253203$

H 2.8550920 .2304870 .998361

C $3.100404-0.336827-1.075303$

H $2.521986-0.907561-1.824547$

H $3.1465360 .693919-1.454146$

C $4.507232-0.913913-0.956721$

H $5.102488-0.288914-0.266062$

H $5.013857-0.862902-1.934189$

C $4.479699-2.347477-0.442904$ 
H $5.503728-2.736795-0.322323$

H $3.987297-2.990397-1.194666$

C $3.714097-2.4399410 .870826$

H $3.651180-3.4869441 .209481$

H $4.269848-1.8943431 .655252$

C $2.311842-1.8512220 .754555$

H $1.794053-1.9184131 .722394$

H $1.715235-2.4499330 .041035$

C -0.212034-0.2083921.638328

C 0.4651590 .0949452 .828354

C - $1.519273-0.7494621 .721606$

C - $0.109614-0.1222954 .076421$

H 1.4715630 .5200062 .779751

C -2.090629 -0.947696 2.987900

C -1.399456 -0.6432034.155258

H 0.4470200 .1209194 .984591

H -3.099612 -1.363494 3.041577

$\mathrm{H}-1.867091-0.8163765 .127485$

C -2.324196 -1.063722 0.515430

C -3.500749 -0.3814700 .241600$

C $-1.828313-2.016063-0.431127$

C $-4.173562-0.559397-0.983598$

H -3.870699 0.3502990 .964678

C -2.490815 -2.171439-1.677396

H -1.091559 -2.753172 -0.094617

C $-3.658888-1.417504-1.941435$

H $-5.090063-0.000222-1.183821$

$\mathrm{H}-2.207516-2.987368-2.347404$

H -4.173037 -1.545187 -2.897382

$\mathrm{L} \cdot \mathrm{Pd}(\mathrm{PhCl})$

$E=-2092.07871413$

Pd $1.007227-0.068444-0.554880$

P - $1.196759-0.2086250 .080758$

C $-1.494042-1.2973611 .564553$

$\mathrm{H}-2.527487-1.1186831 .914273$

C - $1.336174-2.7854311 .257129$

$\mathrm{H}-0.362545-2.9426760 .762848$

$\mathrm{H}-2.111718-3.1117400 .547020$

C -1.415066 -3.638668 2.520608

$\mathrm{H}-2.436238-3.5733832 .938650$

H -1.253960 -4.698428 2.263329

C $-0.415603-3.1841643 .576679$

H $0.610378-3.3333353 .194974$

$\mathrm{H}-0.506921-3.8028954 .484130$

C - $0.611415-1.7101473 .907641$

$\mathrm{H}-1.601406-1.5698504 .378976$

H $0.134878-1.3765784 .646812$

C -0.515284 -0.8477212 .654982$

H - 0.6919220 .2115992 .902517

H $0.509125-0.9039192 .243180$

C $-2.415528-0.750271-1.221806$ 
H -3.330399 -1.116515 - 0.717500

C -1.812632 -1.886165 -2.057574

H $-0.863481-1.519424-2.488974$

H - $1.539920-2.741424-1.423389$

C -2.752175 -2.333415-3.172369

H -3.664085 -2.773152 -2.728466

H -2.277034 -3.135237 -3.760499

C - $3.140747-1.168590-4.073957$

H -3.853558 -1.497087-4.847629

H - $2.242078-0.811643-4.608720$

C $-3.726322-0.020553-3.261343$

H -3.954862 $0.838294-3.913175$

H - $4.688466-0.339150-2.820414$

C - $2.7886240 .417244-2.140613$

H -3.243956 1.236394 -1.564662

H - $1.8583670 .822665-2.580512$

Cl $3.445238-0.190294-2.565637$

C $3.026913-0.673708-0.915579$

C $3.811398-0.1623400 .155373$

C $2.265914-1.870989-0.724159$

C $3.846994-0.8255931 .365538$

H $4.3937000 .745327-0.004591$

C $2.398696-2.5621110 .511552$

H $1.869545-2.402483-1.592568$

C $3.161794-2.0482931 .538769$

H $4.437137-0.4090692 .185066$

H $1.902828-3.5293130 .625515$

H $3.248809-2.5919432 .482268$

C - 1.9429131 .3498330 .730626

C - 3.2810771 .3478601 .150434

C - 1.1731042 .5178840 .913473

C -3.8620012.4618321.745597

H -3.8850240.446562 1.012488

C - 1.7674203 .6293581 .534246

C - 3.0942273 .6080321 .946382

H -4.908879 2.4338792.056895

H -1.1690024 .5333701 .669592$

H -3.532815 4.4912612 .416795

C 0.2398992 .6467860 .477592

C 1.2223763 .0627181 .380829

C $0.6055032 .426608-0.868440$

C 2.5363913 .2771990 .960257

H 0.9522233 .2128452 .428902

C $1.9221942 .651642-1.285968$

H - $0.1704582 .211184-1.606226$

C $2.8859373 .080555-0.372318$

H 3.2886663 .6021901 .682842

H $2.1879022 .498336-2.333057$

H $3.9113353 .254643-0.706529$

L

$E=-1273.75343859$ 
P $0.4406430 .066211-0.383975$

C 0.8427501 .8463960 .056609

H 1.2400721 .8705971 .088954

C $1.8885032 .464846-0.872211$

H $1.5605602 .339535-1.920590$

H $2.8469611 .932002-0.777311$

C $2.1067303 .947264-0.578300$

H 2.5546984 .0521960 .426645

H $2.8390884 .365014-1.288456$

C $0.8033624 .734257-0.626687$

H $0.4066144 .717212-1.658087$

H $0.9833315 .793333-0.379958$

C - 0.2298814 .1308590 .316412

H 0.1198154 .2416061 .358971

$\mathrm{H}-1.1833094 .6799510 .249591$

C -0.4596222 .6531560 .019459$

$\mathrm{H}-1.1840142 .2302430 .733493$

H -0.913939 $2.544912-0.982601$

C $2.043983-0.839742-0.013477$

H $2.599078-0.2955710 .775649$

C $2.906876-0.883512-1.281971$

H 2.324356 -1.382082 -2.077681

H $3.1148330 .130212-1.654287$

C $4.213891-1.637757-1.059500$

H $4.828204-1.090826-0.321067$

H $4.798945-1.659722$-1.993440

C $3.959538-3.052575-0.554875$

H $4.911528-3.570510-0.353955$

H $3.451231-3.631622-1.347175$

C $3.084491-3.0392210 .692393$

H $2.859785-4.0680871 .018071$

H $3.641312-2.5669401 .522210$

C $1.785414-2.2704670 .469616$

H $1.187830-2.2678421 .392695$

H $1.176446-2.795835-0.290269$

C - $0.554445-0.3160921 .134968$

C $0.000785-0.1512402 .412903$

C -1.913049-0.679668 1.031205

C - $0.752975-0.3314653 .567223$

H 1.0527070 .1328612 .507317

C -2.669766 -0.840963 2.203522

C -2.100992 -0.672410 3.460088

H -0.291456 -0.200917 4.549072

H -3.721533 -1.123551 2.113268

H -2.708004 -0.814005 4.357554

C - $2.590826-0.884501-0.274244$

C $-3.733182-0.141248-0.595879$

C $-2.130183-1.837947-1.191643$

C $-4.391898-0.335662-1.808658$

H - 4.0957420 .6108290 .109638

C - $2.791482-2.038304-2.399411$

H -1.248781-2.433403-0.944907 
C -3.922415 -1.284940 -2.714069 H -5.275548 $0.260970-2.047960$

$\mathrm{H}-2.420521-2.788778-3.101540$

H -4.437843 -1.439312 -3.665056

$\mathrm{PtBu}_{3}$

$\mathrm{L}_{2} \cdot \mathrm{Pd}$

$E=-1755.58897069$

Pd $0.000126-0.0010580 .000355$

P -2.299992 -0.000274-0.000130

C -2.977291 0.0383981 .795751

C -2.101280 1.0132532.597970

C -4.449997 0.4155491 .957905

C - $2.738747-1.3370292 .427627$

$\mathrm{H}-1.0351740 .7665592 .465364$

H -2.239992 2.0614282 .312601

H -2.356530 0.9235373 .667714

H -5.122311 -0.255038 1.406516

$\mathrm{H}-4.7243860 .3526753 .024982$

H -4.6560361.4449381.635112

$\mathrm{H}-2.932351-1.2642353 .510791$

H -3.403021 -2.115255 2.030895

$\mathrm{H}-1.694281-1.6584552 .293440$

C $-2.976381-1.575010-0.864914$

C -4.448965 -1.904281-0.619760

C - $2.737040-1.434078-2.371811$

C - $2.100316-2.757036-0.421501$

$\mathrm{H}-4.654799-2.1403320 .432934$

H -5.121183-1.091137-0.923903

H -4.723422 -2.796428 -1.208521

$\mathrm{H}-1.692419-1.156973-2.582106$

$\mathrm{H}-2.930122-2.408416-2.850770$

$\mathrm{H}-3.401070-0.701373-2.847770$

$\mathrm{H}-2.354767-3.638475-1.034576$

$\mathrm{H}-1.034152-2.518433-0.567771$

H -2.239853 -3.034409 0.628719

C $-2.9766311 .535928-0.931474$

C - $2.7387022 .770470-0.055574$

C -2.099997 $1.743999-2.176310$

C $-4.4489501 .487373-1.339991$

$\mathrm{H}-3.4027262 .8151210 .817019$

$\mathrm{H}-1.6941442 .8153680 .289509$

H -2.932987 $3.672353-0.659629$

H -2.238499 $0.973394-2.941979$

H -2.354923 $2.715662-2.632872$

$\mathrm{H}-1.0340001 .752133-1.895937$

$\mathrm{H}-4.7237002 .443178-1.818349$

$\mathrm{H}-4.6540570 .693628-2.070836$

H -5.121363 $1.343848-0.483971$

P $2.300172-0.0001230 .000136$

C $2.978238-1.1517151 .378111$ 
C $2.738564-0.4731952 .730929$

C $2.103705-2.4151601 .394823$

C $4.451383-1.5457891 .269826$

H 3.4011670 .3836362 .907491

H $1.693383-0.1407332 .827195$

H $2.933813-1.2065823 .530968$

H $2.243203-3.0546010 .516735$

H 2.359722 -3.013683 2.285762

H $1.037249-2.1410371 .445572$

H $4.726240-2.1620042 .143105$

H $4.658544-2.1488730 .375591$

H $5.122574-0.6768771 .257523$

C 2.9743821 .7704010 .308658

C $2.7344672 .601890-0.955843$

C 2.0971822 .4152681 .393025

C 4.4467791 .8767410 .706053

H $3.3984442 .327497-1.785418$

H $1.6897822 .516841-1.292877$

H $2.9273103 .661891-0.720818$

H 2.235246 1.9744542 .385920

H 2.3518153 .4863941 .466565

H 1.0314072 .3208591 .128096

H 4.7196982 .9417390 .801874

H 4.6533331 .4056701 .676510

H $5.1198041 .431600-0.038592$

C $2.977492-0.616852-1.686757$

C $2.740855-2.128198-1.774826$

C $2.100527-0.001913-2.788683$

C $4.449634-0.322697-1.975365$

H $3.405714-2.708036-1.121974$

H $1.696549-2.379903-1.533308$

H $2.935148-2.454233-2.810187$

H $2.2373391 .078490-2.903833$

H $2.356854-0.473902-3.752571$

H $1.034689-0.185409-2.575754$

H $4.724855-0.770917-2.945543$

H $4.6539420 .753749-2.051442$

H $5.122497-0.744155-1.216862$

$L \cdot P d(d b a)$

$E=-1672.06760782$

Pd $-0.565997-0.563636-0.815226$

P -0.527920 $1.653223-0.118940$

O $1.160250-3.232242-2.514214$

C $0.800536-2.855182-1.408251$

C $-0.629201-2.698886-1.084591$

$\mathrm{H}-1.294598-3.010010-1.895528$

C - $1.139911-2.3585970 .180041$

H -0.477215 -2.403035 1.050625

C $-2.566806-2.3230640 .536987$

C -2.924646 -2.152630 1.883680

H -2.134764 -2.092653 2.637662 
C -4.929037 -2.309217 -0.032034

H -5.714018 -2.373042 - 0.789487

C - $3.597079-2.406974-0.415285$

H -3.347725 -2.532068 - 1.470731

C $1.791190-2.396654-0.392348$

H $1.465392-2.2701530 .641611$

C $3.042911-2.068276-0.761008$

H $3.284379-2.198624-1.822037$

C $4.074038-1.4807290 .088823$

C $3.962022-1.4093671 .488946$

H $3.098238-1.8558441 .985576$

C $4.938925-0.7818602 .251643$

H $4.833135-0.7400253 .338547$

C $6.055636-0.2104271 .636657$

H 6.8221100 .2832372 .238665

C $6.189444-0.2839840 .251365$

H $7.0625410 .153251-0.238939$

C $5.211739-0.916847-0.511455$

H $5.316401-0.970616-1.598308$

C 0.9383931 .9385711 .081640

C 2.2452921 .7958370 .293115

C 0.9346003 .2756331 .823185

C 0.9458270 .7868432 .097786

H $2.2661360 .858062-0.282508$

H $2.4340002 .637586-0.385281$

H 3.0852461 .7542891 .005496

H 0.0799843 .3728062 .506327

H 1.8481533 .3475522 .437197

H 0.9278814 .1376151 .143410

H 1.8741990 .8456722 .689477

H 0.1017710 .8109182 .794836

H $0.932695-0.1810891 .575795$

C - 2.1534032 .1936700 .732691

C -2.3729953 .7047310 .816267$

C $-3.3185021 .532451-0.018673$

C - 2.1882841 .6043602 .146068

H - 1.5656724 .2192311 .354105

H - $2.4737924 .170230-0.173007$

H -3.311087 3.9018711 .362052

H -3.181104 0.442145 -0.075250

H - -4.2517751 .7288160 .535068$

H -3.453755 $1.911985-1.036889$

$\mathrm{H}-3.1996821 .7522052 .558667$

$\mathrm{H}-1.9966890 .5216042 .133140$

$\mathrm{H}-1.4818602 .0887052 .832156$

C -0.259139 2.715094 -1.689483

C $0.7304181 .948636-2.582269$

C - $1.5698812 .768440-2.481671$

C $0.2451584 .139664-1.460083$

H $1.7357101 .867250-2.156975$

H $0.3694690 .924990-2.786423$

H $0.8169982 .469798-3.550567$ 
H -2.339928 $3.386783-2.003808$

$\mathrm{H}-1.3616283 .211888-3.469324$

H -1.980230 $1.761037-2.649522$

H $0.3187674 .657737-2.431306$

H - $0.4308944 .727518-0.824873$

H $1.2452854 .161974-1.007497$

C -4.258774-2.049775 2.267592

H $-4.511107-1.9118263 .321796$

C $-5.268700-2.1244921 .310278$

H -6.316948 -2.042719 1.606551

L.Pd

$E=-941.139819230$

Pd $-2.077150-0.0004730 .000896$

P $0.1420970 .000039-0.000206$

C $0.8281421 .377757-1.155100$

C $0.5969190 .950050-2.607870$

C - $0.0316732 .634024-0.953618$

C $2.3032541 .736773-0.968196$

H $1.2620180 .138926-2.930365$

H - $0.4468870 .638780-2.767456$

H $0.7953301 .813922-3.263725$

H 0.0941093 .0961130 .031163

H $0.2523313 .383383-1.711993$

H -1.098975 2.392111 -1.078556

H 2.586885 2.490914 -1.722029

H 2.5051442 .1788210 .016506

H $2.9714840 .875981-1.099712$

C $0.829179-1.688527-0.616120$

C $0.599658-2.7327150 .481130$

C $-0.031401-2.142958-1.804009$

C $2.304095-1.705814-1.021388$

H $1.265923-2.6058601 .343883$

H - $0.443691-2.7155320 .831852$

H $0.797970-3.7326460 .060908$

H $0.092654-1.520880-2.696640$

H $0.253363-3.174100-2.074210$

H - $1.098399-2.131291-1.530782$

H $2.587866-2.735841-1.296955$

H $2.505721-1.074790-1.897121$

H $2.972521-1.388714-0.210581$

C 0.8298540 .3114181 .769841

C 0.5984831 .7833722 .125927

C - $0.028724-0.4914172 .758011$

C $2.305353-0.0294171 .986499$

H 1.2639002 .4682131 .585044

H -0.445229 2.077295 1.935787

H 0.7965391 .9193443 .202058

H $0.096710-1.5752842 .665149$

H $0.257052-0.2097823 .785791$

H -1.096233 -0.261986 2.612961

H 2.5894890 .2473363 .016124 
H $2.508029-1.1032471 .877986$ H 2.9729790 .5147171 .306118

$\mathrm{L} \cdot \mathrm{Pd}(\mathrm{PhCl})$

$E=-1632.69884924$

Pd $-0.936140-0.3184220 .050589$

P $1.340042-0.007691-0.011164$

Cl -3.379706 -2.196287 0.045949

C -3.047301-0.4716850.010980

C -2.834847 0.2324311 .226380

C -3.2707940.248510-1.191088

C -2.935066 1.6387651 .224769

$\mathrm{H}-2.778655-0.3160232 .168611$

C -3.357700 $1.636568-1.153459$

$\mathrm{H}-3.434927-0.303713-2.117816$

C -3.215043 2.333362 0.054682

$\mathrm{H}-2.8107452 .1740112 .169107$

H -3.548028 2.181254-2.081109

H -3.307308 3.4210930 .072266

C $1.7299181 .539940-1.076731$

C $0.6909632 .620114-0.736399$

C $3.1382822 .116594-0.928196$

C $1.4842361 .187360-2.547529$

H - $0.3322012 .222440-0.828287$

H 0.8004673 .0286890 .273510

H $0.8076573 .456231-1.446643$

H $3.9232011 .385241-1.161539$

H $3.2573702 .961733-1.627372$

H 3.3254752 .5082330 .080610

H $1.5162372 .116856-3.139598$

H $2.2425850 .511973-2.963583$

H $0.4893600 .737005-2.687213$

C $2.221067-1.522715-0.786382$

C $3.655700-1.296148-1.264158$

C $2.207274-2.6661180 .233666$

C $1.354792-2.008504-1.958939$

H $3.713453-0.574371-2.089770$

H $4.318869-0.948920-0.460833$

H $4.065543-2.248752-1.640879$

H $1.195019-2.8346960 .631718$

H $2.525148-3.591713-0.274068$

H $2.895959-2.5038551 .072515$

H $1.758096-2.967539-2.325698$

H $0.315266-2.173103-1.631927$

H $1.334766-1.314070-2.805254$

C 2.0425760 .2731301 .751142

C 1.5995511 .6594072 .230490

C $1.355381-0.7272792 .692581$

C 3.5603190 .1536661 .892918

H 2.1263602 .4784611 .724622

H 0.5154541 .7977872 .097406

H 1.8214881 .7450233 .307050 
H $1.657351-1.7659372 .522483$

H $1.616771-0.4721153 .733404$

H $0.260972-0.6709402 .581908$

H 3.8445120 .3838102 .933903

H $3.922003-0.8612041 .680146$

H 4.1034420 .8523961 .243191

L

$E=-814.378963896$

P $0.000066-0.000168-0.711872$

C -1.303859-1.224707 -0.001176

C -1.249057 -2.479796 -0.887781

C -1.165574 -1.628805 1.466930

C $-2.703407-0.637267-0.216750$

$\mathrm{H}-1.353864-2.214893-1.950750$

$\mathrm{H}-0.322602-3.053086-0.774233$

$\mathrm{H}-2.084349-3.149061-0.619174$

$\mathrm{H}-1.206581-0.7660972 .144730$

$\mathrm{H}-1.994974-2.3042151 .741232$

$\mathrm{H}-0.232225-2.1715621 .666649$

H -3.450650 -1.421336 -0.008065

$\mathrm{H}-2.9249010 .2050950 .450911$

H -2.852072 -0.308881 - 1.256640

C $-0.4087731 .741340-0.001317$

C -0.8279251 .8243241 .466855$

C $0.7997332 .659617-0.217144$

C - $1.5231952 .321088-0.888033$

$\mathrm{H}-1.7646291 .2874641 .667227$

H -0.060199 1.4292522 .145077

H - 0.9983132 .8804871 .740385

H $1.1585072 .623851-1.257008$

H $0.4944083 .698855-0.008719$

H 1.6399622 .4303300 .450582

$\mathrm{H}-1.6851663 .379187-0.619771$

$\mathrm{H}-1.2415012 .279034-1.951020$

H -2.482828 $1.805352-0.774132$

C $1.712593-0.516825-0.001185$

C $1.903900-2.022572-0.216785$

C $2.7719710 .158439-0.887790$

C $1.993435-0.1947421 .466907$

H $1.285389-2.6357190 .450947$

H $1.693739-2.315617-1.256633$

H $2.956635-2.277432-0.008263$

H $2.8051691 .247402-0.773939$

H $3.769281-0.230336-0.619465$

H $2.594773-0.064479-1.950793$

H $2.993415-0.5745901 .740933$

H 1.9961550 .8849981 .666524

H $1.267383-0.6620522 .145106$ 


\section{References}

[1] Gaussian 09, Revision E.01, Frisch, M. J.; Trucks, G. W.; Schlegel, H. B.; Scuseria, G. E.; Robb, M. A.; Cheeseman, J. R.; Scalmani, G.; Barone, V.; Mennucci, B.; Petersson, G. A.; Nakatsuji, H.; Caricato, M.; Li, X.; Hratchian, H. P.; Izmaylov, A. F.; Bloino, J.; Zheng, G.; Sonnenberg, J. L.; Hada, M.; Ehara, M.; Toyota, K.; Fukuda, R.; Hasegawa, J.; Ishida, M.; Nakajima, T.; Honda, Y.; Kitao, O.; Nakai, H.; Vreven, T.; Montgomery, J. A., Jr.; Peralta, J. E.; Ogliaro, F.; Bearpark, M.; Heyd, J. J.; Brothers, E.; Kudin, K. N.; Staroverov, V. N.; Kobayashi, R.; Normand, J.; Raghavachari, K.; Rendell, A.; Burant, J. C.; Iyengar, S. S.; Tomasi, J.; Cossi, M.; Rega, N.; Millam, J. M.; Klene, M.; Knox, J. E.; Cross, J. B.; Bakken, V.; Adamo, C.; Jaramillo, J.; Gomperts, R.; Stratmann, R. E.; Yazyev, O.; Austin, A. J.; Cammi, R.; Pomelli, C.; Ochterski, J. W.; Martin, R. L.; Morokuma, K.; Zakrzewski, V. G.; Voth, G. A.; Salvador, P.; Dannenberg, J. J.; Dapprich, S.; Daniels, A. D.; Farkas, Ö.; Foresman, J. B.; Ortiz, J. V.; Cioslowski, J.; Fox, D. J. Gaussian, Inc., Wallingford CT, 2009.

[2] (a) Hohenberg, P.; Kohn, W. Inhomogeneous Electron Gas. Phys. Rev. 1964, 136, B864-B871. (b) Kohn, W.; Sham, L. J.; Self-Consistent Equations Including Exchange and Correlation Effects. Phys. Rev. 1965, 140, A1133-A1138.

[3] Adamo, C.; Barone, V. Toward reliable density functional methods without adjustable parameters: The PBE0 model J. Chem. Phys. 1999, 110, 6158-6170.

[4] (a) Hay, P. J.; Wadt, W. R. Ab initio effective core potentials for molecular calculations. Potentials for the transition metal atoms Sc to Hg. J. Chem. Phys. 1985, 82, 270-283. (b) Hay, P. J.; Wadt, W. $\mathrm{R}$. Ab initio effective core potentials for molecular calculations. Potentials for main group elements Na to Bi. J. Chem. Phys. 1985, 82, 284-298. (c) Hay, P. J.; Wadt, W. R. Ab initio effective core potentials for molecular calculations. Potentials for $\mathrm{K}$ to Au including the outermost core orbitals. $J$. Chem. Phys. 1985, 82, 299-310.

[5] Weigend, F.; Ahlrichs, R. Balanced basis sets of split valence, triple zeta valence and quadruple zeta valence quality for $\mathrm{H}$ to $\mathrm{Rn}$ : Design and assessment of accuracy. Phys. Chem. Chem. Phys. 2005, 7, 3297-3305.

[6] (a) Grimme, S.; Antony, J.; Ehrlich, S.; Krieg, H. A consistent and accurate ab initio parametrization of density functional dispersion correction (DFT-D) for the 94 elements H-Pu. J. Chem. Phys. 2010, 132, 154104-154119. (b) Grimme, S.; Ehrlich, S.; Goerigk, L. Effect of the damping function in dispersion corrected density functional theory. J. Comput. Chem. 2011, 32, 1456-1465. (c) Smith, D. G. A.; Burns, L. A.; Patkowski, K.; Sherrill, C. D. Revised Damping Parameters for the D3 Dispersion Correction to Density Functional Theory. J. Phys. Chem. Lett. 2016, 7, 2197-2203.

[7] Deglmann, P.; Furche, F.; Ahlrichs, R. An efficient implementation of second analytical derivatives for density functional methods. Chem. Phys. Lett. 2002, 362, 511-518.

[8] Zhao, Y.; Truhlar, D. G. The M06 suite of density functionals for main group thermochemistry, thermochemical kinetics, noncovalent interactions, excited states, and transition elements: two new functionals and systematic testing of four M06-class functionals and 12 other functionals. Theor. Chem. Acc. 2008, 120, 215-241.

[9] Gaussian NBO Version 3.1, Glendening, E. D.; Reed, A. E.; Carpenter, J. E.; Weinhold, F.

[10] (a) Becke, A. D. Density-functional thermochemistry. III. The role of exact exchange. J. Chem. Phys. 1993, 98, 5648-5652. (b) Lee, C.; Yang, W.; Parr, R. G. Development of the Colle-Salvetti correlation-energy formula into a functional of the electron density. Phys. Rev. B 1988, 37, 785789. (c) Vosko, S. H.; Wilk, L.; Nusair, M. Accurate spin-dependent electron liquid correlation energies for local spin density calculations: a critical analysis. Can. J. Phys. 1980, 58, 1200-1211; (d) Stephens, P. J.; Devlin, F. J.; Chabalowski, C. F.; Frisch, M. J. Ab Initio Calculation of Vibrational Absorption and Circular Dichroism Spectra Using Density Functional Force Fields. J. Phys. Chem. 1994, 98, 11623-11627.

[11] (a) McLean, A. D.; Chandler, G. S. Contracted Gaussian basis sets for molecular calculations. I. Second row atoms, Z=11-18. J. Chem. Phys. 1980, 72, 5639-5648. (b) Raghavachari, K.; Binkley, J. S.; Seeger, R.; Pople, J. A. Self-consistent molecular orbital methods. XX. A basis set for correlated wave functions. J. Chem. Phys. 1980, 72, 650-654.

[12] (a) Andrae, D.; Häussermann, U.; Dolg, M.; Stoll, H.; Preuss, H. Energy-adjustedab initio pseudopotentials for the second and third row transition elements Theor. Chim. Acta 1990, 77, 123- 
141. (b) Häussermann, U.; Dolg, M.; Stoll, H.; Preuss, H.; Schwerdtfeger, P.; Pitzer, R. M. Accuracy of energy-adjusted quasirelativistic ab initio pseudopotentials. Mol. Phys. 1993, 78, 1211-1224. (c) Leininger, T.; Nicklass, A.; Stoll, H.; Dolg, M.; Schwerdtfeger, P. The accuracy of the pseudopotential approximation. II. A comparison of various core sizes for indium pseudopotentials in calculations for spectroscopic constants of InH, InF, and InCl. J. Chem. Phys. 1996, 105, 10521059. (d) Küchle, W.; Dolg, M.; Stoll, H.; Preuss, H. Energy-adjusted pseudopotentials for the actinides. Parameter sets and test calculations for thorium and thorium monoxide. J. Chem. Phys. $1994,100,7535-7542$. 